
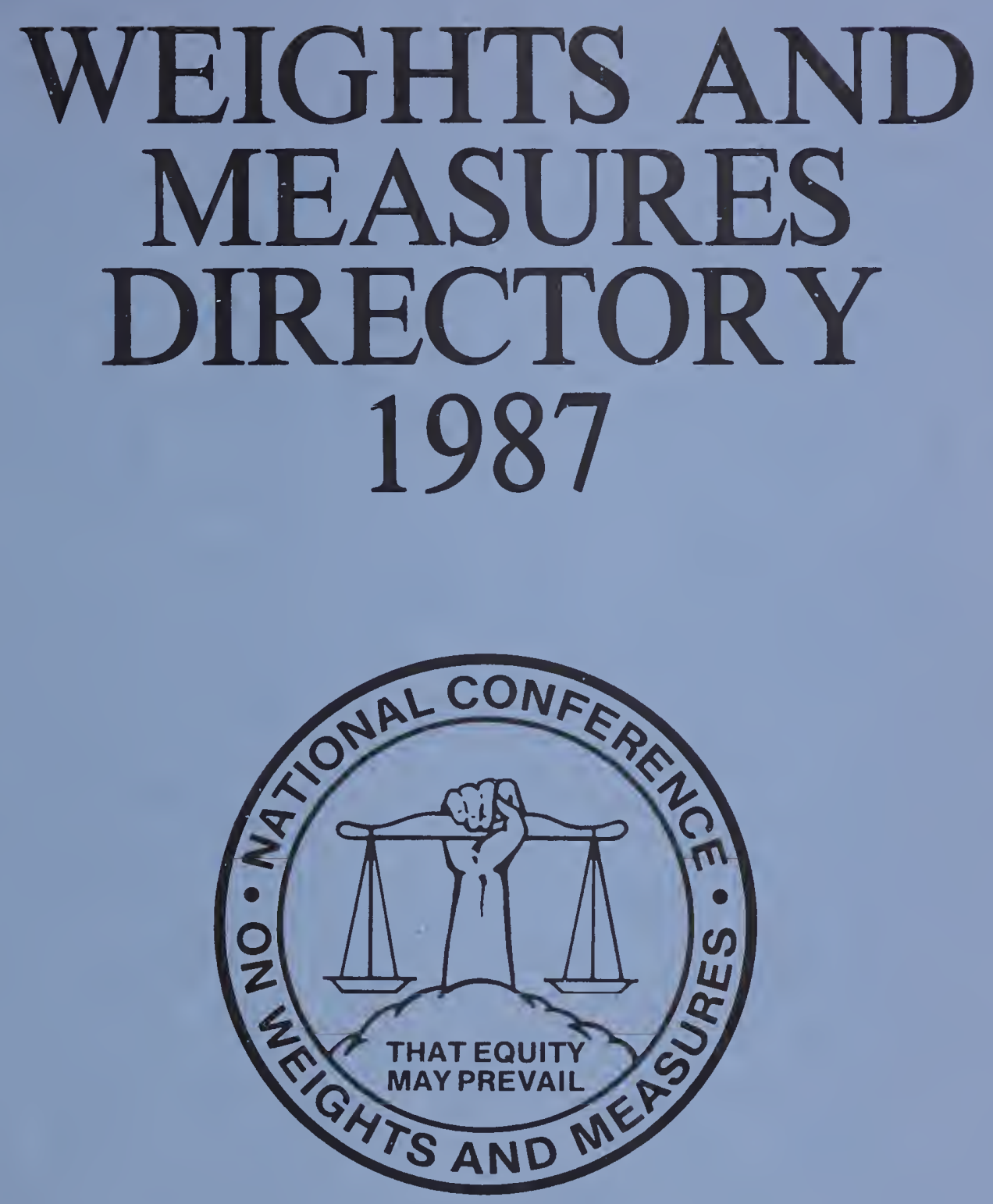

NCWM Publication 2

NESIR $87-3582$

U.S. Department of Commerce National Bureau of Standards 

Please substitute this page in the Weights and Measures Directory 1987

FEDERAL GOVERNMENT MEMBERS

National Bureau of Standards

Karen L. Barkley

Office of Weights \& Measures National Bureau of Standards Admin. Bldg. A617

Gaithersburg, MD 20899 301/975-4004

Carroll S. Brickenkamp office of Weights \& Measures Nationa'l Bureau of Standards Admin. Bldg. A617

Gaithersburg, MD 20899

301/975-4005

Wanda Capino

Off. Product Stds. Policy

National Bureau of Standards

Admin. Bldg. A603

Gaithersburg, MD 20899

301/975-4002

David E. Edgerly

office of Research

\& Technical Activities

National Bureau of Standards

Admin. Bldg. A402

Gaithersburg, MD 20899

301/975-3087

Stephen Hasko

(Retired)

Office of Weights \& Measures

National Bureau of Standards

Admin. Bldg. A617

Gaithersburg, MD 20899

301/975-4006

Ann P. Heffernan

Conference Coordinator

Nat 1. Conf. Weights \& Meas.

P.O. Box 3137

Gaithersburg, MD 20878

301/975-4012

Joan A. Koenig

Office of Weights \& Measures

National Bureau of Standards

Admin. Bldg. A617

Gaithersburg, MD 20899

301/975-4007

Paul H. Krupenie

Office of Weights \& Measures

National Bureau of Standards

Admin. Bldg. A617

Gaithersburg, MD 20899

301/975-4015
Walter G. Leight

Off. Product Stds. Policy

National Bureau of Standards

Admin. Bldg. A603

Gaithersburg, MD 20899

301/975-4010

Karl G. Newell

Office of Weights \& Measures

National Bureau of Standards Admin. Bldg. A617

Gaithersburg, MD 20899

301/975-4013

Henry V. Oppermann

Office of Weights \& Measures

National Bureau of Standards

Admin. Bldg. A617

Gaithersburg, MD 20899

$301 / 975-4008$

Richard N. Smith

Office of Weights \& Measures

National Bureau of Standards

Admin. Bldg. A617

Gaithersburg, MD 20899

301/975-4014

Albert D. Tholen

Chief, Office of Weights

and Measures

National Bureau of Standards

Admin. Bldg. A617

Gaithersburg, MD 20899

301/975-4009

Eric A. Vadelund

Standards Management Program

National Bureau of Standards

Admin. Bldg. A625

Gaithersburg, MD 20899

$301 / 975-4028$

Otto K. Warnlof

Standards Management Program National Bureau of Standards Admin. Bldg. A625

Gaithersburg, MD 20899

$301 / 975-4026$

Stanley I. Warshaw

Director, Off. of Product

Standards Policy

National Bureau of Standards

Admin. Bldg. A603

Gai thersburg, MD 20899

301/975-4001 



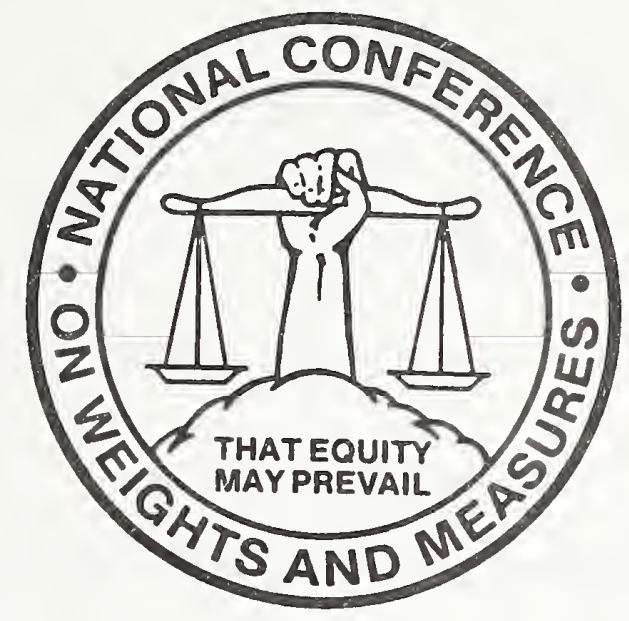

1987

\section{WEIGHTS AND MEASURES DIRECTORY}

This Directory contains a listing of technical staff of all weights and measures jurisdictions in the United states. The names, addresses, and phone numbers have been thoroughly checked with the Directors of each State. However, due to reorganizations, retirements, and position changes implemented after this printing, some information may no longer be current. If a change, deletion, or addition is required, please send updated information to:

Ann P. Heffernan

Conference Coordinator

National Conference on Weights and Measures

P.Q. Bax 3137

Gaithersburg, MD 20878

This issue contains the designation "MBR NCWM" to the right of the names of the persons who are 1987 members. If you are not now a member and would like to join the National Conference on Weights and Measures, an application is provided in the back of this publication. Please fill it out and send to the above address along with your check for $\$ 35.00$ made payable to the National Conference on Weights and Measures.

For convenience of use, State Directors are 1 isted together in the first section of the Directory. National Bureau of Standards members along with other U.S. Government members are listed in SECTION III. 



\section{TABLE DF CONTENTS}

\section{SECTION I}

PAGE NUMBER

STATE WEIGHTS AND MEASURES DIRECTORS. . . . . . . . . . . . 1

\section{SECTION I I}

STATE, COUNTY, AND CITY WEIGHTS AND MEASURES OFFICES. . . . 7 STATE

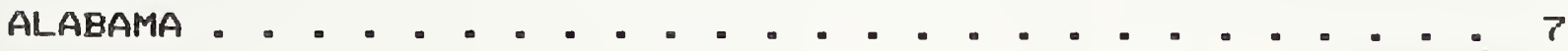
ALASKA. . . . . . . . . . . . . . . . . . . . . . . . . 8 AMERICAN SAMDA. . . . . . . . . . . . . . . . . . . . . . 8 ARIZONA - . . . . . . . . . . . . . . . . . . . . . . . . . 8 ARKANSAS. . . . . . . . . . . . . . . . . . . . . . . . . . 9 CALIFORNIA. - . . - . . . - . . . . - . . . . . . . . 10 COLDRADO. . . . . . . . . . . . . . . . . . . . . . . . . 28 CONNECTICUT . . . - . . . . . . . . . . . . . . . . . . . . 28 DELAWARE. . . . . . . . . . . . . . . . . . . . . . . . . . 30 DISTRICT OF CULUMBIA. . . . . . . . . . . . . . . . . . . . 30 FLORIDA - . . . . . . . . . . . . . - . . . . . . . 31 GEORGIA - . . . . . . . . . . . . . . . . . . . . . . . 35 GUAM. . . . . . . . . . . . . . . . . . . . . . . . 35 HAWAI I. . . . . . . . . . . . . . . . . . . . . . . . 36 IDAHO . . . . . . . . . . . . . . . . . . . . . . . . 36 ILLINOIS. - . . . . . . . . . - . . . . . . . . . . 37 INDIANA . . . . . . . . . . . . . . . . . . . . . . 39 
IOUA. . . . . . . . . . . . . . . . . . . . . 43

KANSAS. .............................. 43

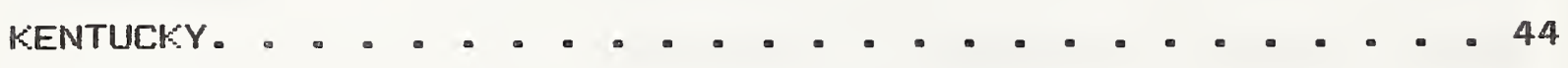

LOUISIANA . . . . . . . . . . . . . . . . . . . . . 45

MAINE.................................. 46

MARYLAND. . . . . . . . . . . . . . . . . . . . . . 48

MassachusetTS . . . . . . . . . . . . . . . . . . . . . . . 50

MICHIGAM. . . . . . . . . . . . . . . . . . . . . . . 62

MINNESOTA . . . . . . . . . . . . . . . . . . . . . . 66

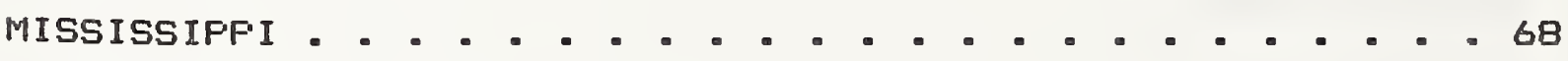

MISSOURI. . . . . . . . . . . . . . . . . . . . . 69

MONTANA -................................ 71

NEBRASKA. - . . . . . . . . . . . . . . . . . . . . . 72

NEVADA. . . . . . . . . . . . . . . . . . . . . . . 72

NEW HAMPSHIRE - . - . . . . . . . . . . . . . . . . 73

NEW JERSEY. - . . . . . . . . . . . . . . . . . . . . . . 73

NEW MEXICO. . . . . . . . . . . . . . . . . . . . 78

NEW YORK. . . . . . . . . . . . . . . . . . . . . . . . . 79

NORTH CAROLINA. - . - . - . . - . . . . . . . . . . . . 85 NORTH DAKOTA. ............................ 86

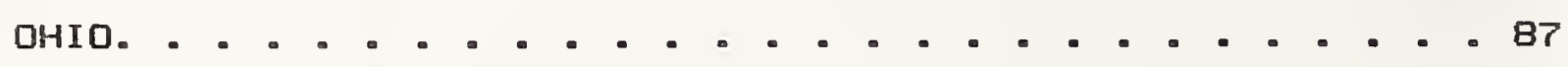
OKLAHOMA. . . . . . . . - . . . . . . . . . . . . . . . 98 OREGON. - . . . . . . . . . . . . . . . . . . . . . . . 99 PENISYLVANIA. - . . . . . . . . . . . . . . . . . . . . 100 
PUEFITO RICO . . . . . . . . . . . . . . . . . . . . 107

RHODE ISLAND.................................. 10? SOUTH CAROLINA. . . . . . . . . . . . . . . . . . . . 109 SOUTH DAKOTA. . . . .......................... 110 TENNESSEE . . . . . . . . . . . ............. 110 TEXAS ............................... 112 UTAH. - . . . . . . . . . . . . ............ . 113 VERMONT ............................. . . 114

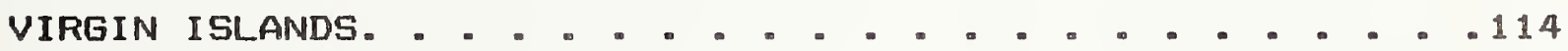
VIRGINIA. . . . . . . . . . . . . . . . . . . . . . 114 WASHINGTON. . . . . . . . . . . . . . . . . . . . . . . 116 WEST VIFGINIA - . . . . . . . . . . . . . . . . . . 117 WISCONSIN . . . . - . . . . . . . . . . . . . . . 117 WYOMING .............................. 119

\section{SECT ION II I}

FEDERAL GQVERNMENT MEMBERS. . . . . . . . . . . . . . . . . 121 National Bureau of Standards... . . . . . . . . . 121 Dther Government Agencies. . . . . . . . . . . . . . 123

NCWM MEMBERSHIP APPLICATION - . . . . . . . . . . . . . . 125 
SECTION I

STATE HEIGHTS AND MEASURES DIRECTORS 

DON E STAGG

DIRECTOR

WEIGHTS 6 MEASURES DIV

P.0. BOX 3336

MONTGOHERY AL 36193

$205261-2613$

AL ASKA

JOSEPH 2 SHANSON

DIRECTOR DIVISION MEASURE STOS

AL ASKA DEPT COHMOECON DEVELOP 12050 IND UY BLD O/POB L11686 ANCHORAGE, AK 99511

$907345-7750$

\section{AHERICAN SAMOA}

ARTHUR L YOUNG

SUPERUISOR

HEIGHTS AND MEASURES DIUISION

AMERICAN SAMOA GOUERNMENT

PAGOPAGO AMER, IS 96799

\section{AR IZONA}

RAYMOND H HELAICK

CHIEF

WEIGHTS 6 NEASURES

3039 WEST TNDEAN SCHOOL RD

PHOENIX, AZ 85017

602 255-5211

ARKANSAS

\section{SAM F HINDSNAN}

DIRECTOR

ARKANSAS BUREAU OF STANDARDS

4608 H 61ST ST

LITTLE ROCK, AR 72209

$501371-1736$
DARRELl A GUENSLER

CHIEF

CAL IF DIV OF MEASUREMENT STO

8500 FRUITRIDGE RD

SACRAMENTO. CA 95826

$916 \quad 366-5119$

COLORADO

dAVI D waLlaCe

CHIEF MEASURMNT STANDARDS SECT DEPARTMENT OF AGRICULTURE

3125 WYANDOT

DENYER, CO 80220

$303866-2845$

CONNECTICUT

ALlan m NELSON

CHIEF WEIGHTS 6 MEASURES

DEPT OF CONSUMER PROTECTION

165 CAPITOL AVE ROOA GI7

HARTFORD, CT 06106

$203566-5230$

DELAWARE

EUGENE KEELEY

SUPERVISOR

DELAWARE WEIGHTS AND MEASURES 2320 SOUTH DUPONT HIGHHAY

DOVER, DE 19903

$302736-4811 \times 37$

FLOR IDA

HAYNE BALL

CHIEF BUREAU OF HEIGHTS \& MEAS

FLA DEPT OF AGRIC \& C $S$

3125 CONNER BLVD

TALLAHASSEE。FL 323991650

$904488-9140$ 
BILL TRUAV

OIRECTOR FUEL 6 MEASURES DIV

GA DEPT OF AEALCULTUE

AGRI BLDG CAPITAL SO

ATLANTA. GA 30334

404 656-3605

GUAM

JUAN REYES UWPINGO

SUPERUI SAR-HEIGHTS O MEASUAES

GUAM DEPT REVENUE TTAKATION

P O BOX 3663

agana guAHe GU 98910

HAWAII

GEORGE e MATTIMDE

ADAINISTRATOR MEASUREMENT STOS DEPT OF $A G R I C U T T U R E$

725 ILALO ST

HONOLULU, HI 98813

808 548-7152

IOAHO

DALE hURD

CHIEF

BUREAU OF HEIENTS AM HEASURES

2216 KELlOGG LANE

BOISE, ID 83702

$208334-2345$

\section{ILLINOIS}

SIONEY A COLBROOK

W 6 A PROGRAN MANAGER

ILLINOIS DEPT OF MBRICMITAE

801 EAST SANBAMON AUEMUE

SPRINGFIELD, IL 62706

$217782-3817$
ROBERT W HALKER

DIRECTOR

HEIGHTS AND MEASURES DIVISION

1330 U MICHIGAN ST

INDIANAPOLIS. IN 46206

317 633-0350

IOHA

JAMES M OCONNDR

SUPERVISOR

WEIGHTS ANO MEASURES

HENRY A HALLACE BLDG

DES MOINES. IA 50319

$51528 L-5716$

KANSAS

ARCHIE HURST

ACTING STATE SEALER G DIRECTOR KANSAS HEIGHTS 6 MEASURES

2016 SW 37TH ST

TOPEKA. KS 666112570

$913 \quad 267-4641$

RENTUCKY

CHARLES l PREBble DIRECTOR

DIVISION OF WEIGHTS $\&$ MEASURES 106 WEST SECOND STREET

FRANKFORT, KY 40601

$502564-4870$

LOUISIANA

RONALD HARRELL

DIRECTOR OF WTS \& MEASURES

LA DEPT OF AGR ICULTURE

P O BOX 44456 CAPITOL STATION BATON ROUGE. LA 70804

504 925-3780 
CLAYTON F DAVIS

DIRECTOR

AGR ICULTURE INSPECTIONS OIV

STATE HOUSE STATION 28

AUGUSTA. HE 04333

$207289-3841$

MARYLAND

RICHARD \& THOMPSON

CHIEF OF WEIGHTS \& MEASURES

MD DEPT OF AGRICULTURE

50 HARRV S TRUMAN PARKWAY

ANNAPOL IS, MO 21401

$30184 L-5790$

MASSACHUSETTS

CHARLES H CARROLL

ASST DIRECTOR OF STANDARDS

MASS DIVISION OF STANDARDS

DNE ASHBURTON PLACE

BOSTON. MA 02108

$617727-3480$

MICHIGAN

EDWARD C HEFFRON

CHIEF FOOD DIVISION

MI DEPT OF AGRIC PO BOX 30017

OTTOWA BLDG N 4TH FLOOR

LANSING. MI 48909

$517373-1060$

MINNESOTA

EDWARO P SKLUZACEK

DIRECTOR

WTS ANO MEASURES DIVISION

2277 HI GHWAY 36

ST PAUL. MN 55113

$612341-7200$
WILLIAM P ELDRIDGE

DIRECTOR WEIGHTS E MEASURES DV HS DEPT OF AGRIC 6 COMMERCE P O BOX 1609

JACKSON, MS 392151609

$601 \quad 359-3670$

MISSOURI

LESTER H BARROWS

DIRECTOR

DIV OF WEIGHTS AND MEASURES

P O BOX 630

JEFFERSON CITY, MO 65102

$314751-4278$

MONTANA

STEPHEN H MELOY

ADM IN ISTRATOR

BUREAU OF HEIGHTS \& MEASURES

1424 9TH AVE

HELENA. MT 59620

$406444-3164$

NEBRASKA

STEVEN A MALONE

DIRECTOR

WEIGHTS AND MEASURES DIVISION 301 CENTENNIAL MALL S BOX94757 LINCOLN, NE 68509

$402471-4292 \times 208$

NEVADA

KNUTE D PENNINGTON

SUPERVISOR WEIGHTS \& MEASURES NEVADA DEPT OF AGRICULTURE PO BOX 11100

RENO. NV 89510

$702789-0166$ 
ROY HOHARD

DIRECTOR

NEW HAMPSHIRE DEPT OF AGRIC

CALLER BOX 2042

CONCDRD, NH 03301

$603271-3700$

NEW JER SEY

THOMAS W RELLY

STATE SUPERINTENDENT

STATE OFFICE OF WEIGHTS \& MEAS

187 WEST HANOVER ST

TRENTON, NJ 08625

$609292-4615$

NEW AEXICO

FRED A GERK

DIR DIY STOS \& CONSUMER SUCS

ANM DEPT OF AGRICULTURE

PO BOX 3170

LAS CRUCES. NM 88003

$505646-1616$

NEW YORK

JOHN J BARTFAI

OIRECTOR

BUREAU OF WEIGHTS \& MEASURES

1220 HASHINGTON AVE

ALBANY. NY 12235

$518 \quad 457-3452$

NORTH CAROLINA

N DAVID SMITH

DIRECTOR STANDARDS DV

NC DEPY OF AGRICULTURE

PO BOX 27647

RALEIGH. NC 27611

$919733-3.313$
NORTH DAKOTA

BRUCE $R$ NIEBERGALL

DIRECTOR

HEIGHTS \& MEASURES DIV

STATE CAPITOL

BISMARCK 。 NO 58505

$701224-2400$

OHIO

BRUCE LITZENBERG

DEPUTY DIRECTOR \& CHIEF

DIVISION OF WEIGHTS \& MEASURES

8995 E MAIN ST

REYNOLDSBURG:OH 43068

614 866-6361

OKL AHOMA

- RAY ELLIOTt

DIRECTOR AGRIC PRODUCTS DIV

OKLA DEPT OF AGRICULTURE

2800 NORTH LINCOLN BLVD

OKLAHOMA CITY. OK 73105

$405521-3861 \times 301$

OREGON

KENDRICK \& SIMILA

ADMINISTRATOR

OREGON HEIGHTS \& MEASURES DIV

635 CAPITOL ST NE

SALEM. OR 97310

$503 \quad 378-3792$

PENNSYLVANIA

FRED A THOMAS

DIRECTOR

PA BUREAU OF WEIGHTS \& MEASURE 2301 N CAMERON ST

HARR ISBURG, PA 17110

$717787-6772$ 
IBONE RWILAN

ASSISTANT SECRETARY

DEPARTMENT OF CONSUMER AFFAIRS

PO BOX 41059 MINILLAS STATION

SANTURCE, PR 00940

809 726-7585

RHODE ISLAND

LYNDA MAURER

SUPERVISING METROLOGIST

DIV OF HEIGHTS \& MEASURES

220 ELMWOOD ROAD

PROVIDENCE, RI 02907

$401457-1863$

SOUTH CAROLINA

CAROL P FULMER

ASSISTANT DIRECTOR

SC DEPT OF AGRICULTURE

P O BOX 11280

COLUMBIA. SC 29211

$803 \quad 737-2080$

SOUTH DAKOTA

JAMES MELGAARD

OIRECTOR

DIV COMM INSP \& REG

128 WEST CAPITOL

PIERRE, SD 57501

$605 \quad 773-3697$

TENNESSEE

JOE R GAINES

OIRECTOR

WEIGHTS AND MEASURES

BOX 40627 MELROSE STATION

NASHVILLE。 TN 37204

$615360-0160$
EDWIN J PRICE

ENFORCEMENT COORD INATOR

DEPT OF AGRICULTURE

PO BOX 12847

AUSTIN. TX 78711

$512463-7607$

UTAH

EDISON J STEPHENS

DEPUTY COMMISSIONER

DEPT OF AGRICULTURE

350 NORTH REDWOOD ROAD

SALT LAKE, UT 84116

$801533-4109$

VERMONT

TRAFFORD F BRINK

DIR WTS \& MEAS \& RETAIL INSP VERMONT DEPT OF AGRICULTURE

116 STATE ST

MONTPELIER • VT 05602

$802828-2436$

VIRGIN ISLANDS

LOUIS PENN

ACTING DIRECTOR

CONSUMER SERYICES DIVISION GOLDEN ROCK SHOPPING CENTER CHRISTIANSTED ST. VI 00820 $809774-3130$

VIRGINIA

JAMES F LYLES

CHIEF

VIRGINIA WEIGHTS \& MEASURES

P O BOX 1163 ROOM 403

RICHMOND, VA 23209

804 786-2476 
HASHINGTON

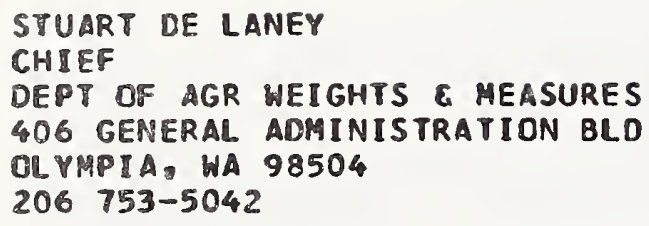


SECTION II

STATE, COUNTY AND CITY

WEIGHTS AND MEASURES OREICES 



\section{STATE}

ANDERS, CAN

WEIGHTS \& MEASURES INSPECTOR

DIV 4 M DEPT OF AGRIC E IND $P$. BOX 3336

MONTGOMERY. AL 36193

AVARITT EOWARD 0

HEAVY DUTY HE M INSPECTOR

DIV $W$ M DEPI OF AGRIC E IND

MONTGOMERY. AL 36193

CAMPBELL J JOHN H

METROLOGISTIST OF AGRIC \& IND PO BOX 3336

PONTGORERY, AL 36193

CROCKER D DON K DIV $W$ \& DEPT OF AGRIC E IND

MONTGOMERY AL 36193

CROMWELL \& EDDIE

WEIGHTS \& MEASURES INSPECTOR DIV $W$ I DEPT OF AGRIC E INO P 0 BOX 3336

MONTGOMERY AL 36193

CRYER HUBTS E MER S TRNG OFFICER DIV $W$ \& DEPT OF AGRIC E IND P O BOX 3336

MONTGOAERY AL 36193

FUL MER R RANDY

HEAVY DUTY H $\%$ INSPECTOR DIV 4 \& DE

MONTGOMERY. AL 36193

GISSENDANNER FRANK

WE IGHTS E MEA SURES INSPECTDR DIV W \& M DEPT OF AGRIC \& IND PO BOX 3336

AL 36193

GIS SENDANNER RDBERT E

HEAVY DUTY W $M$ M INSPECTOR

DIV \& \& DEPT OF AGRIC E IND P D BOX 3336

MONTGOMERY. AL 36193

GOODWIN * LARRY

WEIGHTS \& MEASURES INSPECTOR

DIV $W$ \& DEPT OF AGRIC E IND PO BOX 3336

MONTGOMERY AL 36193

HICKS. R Y

CHIEF GRAIN INSPECTOR

STA GRAIN INSPECTION

GUNTERSVILLE. AL 35976

HOLLIS. STEADMAN L

WEIGHTS \& MEASURES INSPECTOR DIV W E M DEPT OF AGRIC E IND P D BOX 3336

MONTGOMERY. AL 36193
PILKINTON. ${ }^{\circ}$ P DIV $W$ E $A$ OEPT OF AGRIC E INO MONTGOHERY AL 36193

RABB: JOHN

WT ${ }^{\circ}$ OF LABARATORY SUPERVISOR P 0 BOX 3336

MONTGDHERY AL 36193

RABON. PAUL

WEIGHTS \& MEASURES INSPECTOR DIV W E 3336

MONTGOMERY: AL 36193

SELLERS S. JAMES H DEPARTMENT OF AGR IC E IND

MONTGOMERY AL 36193

SNOW- TERRY

DEPT OF AGR ICULTURE

DIV OF WEIGHTS \& MEASURES

P. BOX 3336

MONTGOMERY AL 36193

STAGG DON E

DIRECTOR

HEIGHTS $E$ MEASURES DIV

MONTGOHERY AL 36193

TERRELL JR • HERMAN

HEAVY DUTY HE M INSPECTOR

DIV $W M$ DEPT OF AGRIC E IND P. BOX 3336

MONTGOMERY. AL 36193

WEATHERLY. AUBREY T

WEIGHTS E MEASURES INSPECTOR DIV \& $Y$ DEPT OF AGRIC E IND P O BOX 3336

MONTGOMERY AL 36193

CITY: BIRMINGHAH

BURNS. CHARLES

CHIEF INSPECTOR

CITY OF BIRMINGHAM HEM

CITY HALL ROOH 207

BIRMINGHAH AL 35203

254-2211 $\times 2246$

HAMILTON DONALD

CIIY OF BIRHINGHAM

BIRMINGHAM,AL 35203

HIGGINS, WILLIAM P

WEIGHTS $\&$ MEASURES DIV

ROOM 207 CITY HAL

BIRMINGHAM AL 35203

WILLIAMS: DONALD CIYY OF BIRMINGHAM $E$ M

BIRMINGHAM. AL 35203
HCW

MCHM

MBR

NCWM

MBR

NCWM

MBR
NCWM 

ATCHLEV - WILLIAM H
WEIGHTS \& MEASURES INSPECTOR
CITY OF HUNTSVILLE PO EOX 308

HUNTSVILLE 205 S AL 35804

ALASKA

STATE

BERNSTEIN G GARY

IN SPECTCR TRAINEE

ALASKA DEPT COMA/ECON DEV 1206

ANCHORAGE AK 99511

CAVENDER DARREL DIV OF MEA SUREMENT STANDARDS ANCHORAGE AK 99511

COMISKEY ED FIELD SUPV

ALASKA DEPT COMM/ECON DEV

12050 IND WY BL O/POB 111686 ANCHORAGE, AK 99511

$907345-7846$

EMER ICK DEWEV

ALASKA DEPT COMM/ECON DEV

675 TTH AVE STATION A

FAIRBANKS AK 99701

LANDIS JOHN

INSPEC TCR TRAINEE

AL A SKA DEPT COMM/EC CN DEV

12050 IND WY BL OFOB 111686 ANCHORAGE AK 99511

$907345-7846$

LOVELL JAMES

ALASKA DEPT COMMIECON DEY

12050 IND HY BL OPPOB 111686 ANCHCRAGE AK 99511

$907345-7846$

MERRYMAN, JERRY

INSPECTOR I COMM/ECON DEV

ALASKA DEPT COMM/ECON DEV

$907345-7846$ AK 99511

STEVENSON. RUSSELL

INSPECTOR III SOUTHEAST REGION DEPT OF COMM/ECON DEV

PO BOX 34276

JUNEAU AK 99803

$907789-9763$

SWANSONE JOSEPH L

DIRECTOR DIVISION MEASURE STOS

ALASKA DEPT COMM/ECCN DEVELOP

12050 IND HYY BLD O/POB 111686 ANCHORAGE, AK 99511

907 345-7750
MBR

NCWM

THOMPSON AVES D

ALASKA DEPT COMM/ECON DEV

12050 IND WY BL O/POB 111686

ANCHORAGE AK 99511

YAN YLEET, TOM

INSPECTOR II MEAS ST/STERLING WEIGH STA

$B O X 1216$

KENAI AK 99611

$907262-3853$

\section{AMERICAN SAMOA}

STATE

MBR

YOUNG. ARTHUR L

SUPERVISOR

WEIGHTS AND MEASURES DIVISION

AMER ICAN SAMOA GOVERNMENT

PAGOPAGO AMER, AS 96799

ARI ZONA

MBR

STATE

BIGTHUMB. MEL VIN

WEIGHTS G MEASURES INSPECTOR THE NAVAJO NATION

$P$ BOX 1690

HINDOW ROCK, AZ 86515
$602871-4941$ X 1473

MBR

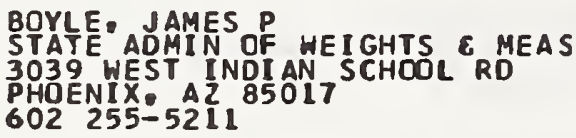

DIETS, JOHN L

STATE ADMIN OF HEIGHTS 6 MEAS 3039 YEST INDIAN SCHOOL RD PHOENIX. AZ 85017

602 255-5211

MBR

FERGUSON JAMES

STATE ADMIN OF WEIGHTS \& MEAS 3039 WEST INDI AN SCHOOL RD PHOENIX AZ 85017

$602255-5211$

MBR

NCWM

FULTON ERNEST

STATE ADMIN OF WEIGHTS E MEAS 3039 HEST INDI AN SCHOOL RD $602255-5211$

MBR

HELMICK, RAYMOND H

WEIGHTS \& MEASURES

3039 HEST INOIAN SCHODL RD

PHOLNIX:AZ 85017

HESSE WINFR IED

WEIGHTS AND MEASURES

MBR

3039 HEST INDIAN SCHOOL RD $602255-5211$

HORN . RI CHARD P

WEIGHTS \& MEASURES OFFICIAL STATE OF ARIZONA

3039 HEST INDI AN SCHOOL RD PHOENIX: 252 Z
MBR

NCWM

MBR

NCWM

MBR

NCWM

MBR

NCGM 
HRTON RUSSELL

ATE ADAIN OF WEIGHTS 6 MEAS PIOENIX, AZ 85017

$2255-5211$

NDIS. MARTIN

IGHTS $\&$ MEASURES

39 WEST INDIAN SCHOCL RD

$62255-5211$

MCEY RCGER

HIGHTS $\&$ MEASURES DIV

DPAP.THENT OF ADYINISTRATION 339 HEST INDIAN SCHDOL RD PCENIX, AZ 85017

$2255-5211$

MUZY J J E 0 Box 3033

IC SON. AZ B5751

(12 $255-5211$

JORE DCN $L$

IGHTS \& MEASURES

39 WE ST INDIAN SCHOOL RD

iIC 2 ENIX: $255-5211$

IPLES DANIEL WEIGHTS $\&$ MEAS 39 WEST INDIAN SCHOOL RD

HOCNIX $255-521$

RTEGA, JOHN B

EIGHTS \& MEASURES

939 WEST INDIAN SCHOOL RD

HOENIX: $255-5211$

DBINSON WILLIAM C

EIGHTS 8 MEASURES DIVISION 039 WEST INDIAN SCHCOL ROAD HOENIX, AZ 85017

$02255-5211$

\section{EELEY SCOTT}

TATE ADMIN OF HEIGHTS MEAS 039 WEST INDIAN SCHDOL RD HOENIX

$02255-5211$

NYDER, DWIGHT

TTATE ADMIN OF WEIGHTS \& MEAS 1039 HEST IND IAN SCHOOL RD

HOENIX:AZ

ORENSEN D L LF WEIGHTS \& MEAS TATE ADMIN DF WEIGHTS E M

PHOENIX AZ B5017

$302255 \div 52$ I 1

STRCH JAMES J

3039 W INDIAN SCHOOL RD

PHOENIX.AZ 85017

$602255-521$

SUTTLE, ROGER L

WE IGHTS \& MEASURES

3039 H INOIAN SCHOGL RO

PHDENIX: AZ 85017

$602255-5211$
TSINAJINNIE BARNEY

WTS \& MEAS INSPECTOR II

MBR NAVAJO NATION

$P$ BOX 1690

WINDOW ROCK, AZ 86515

TULLY, JAMES

STATE ADHIN OF WEIGHTS \& MEAS 3039 WEST INDIAN SCHOOL RD

$602255-52$ I1 85017

VANCE。 CALVIN

STATE ADMIN OF WEIGHTS \& MEAS 3039 WEST INDIAN SCHCOL RD PHOENIX, AZ 85017 $602255-5211$

NBR

WOLFE, RICHARD E

DEPUTY CHIEF

WEIGHTS \& MEASURES

3039 INDIAN SCHOCL RD

$602255-5211$

ARKANSAS

STATE

BAUGUS RAY

BUREAU OF STANDARDS

4608 HEST 6 IST ST

LITTLE ROCK: AR 72209

BEATTY HILTON

INVESTIGATOR II

ARKANSAS BUREAU OF STANDARDS

4608 WEST $615 T$ ST

$501371-1759$ AR 72209

BISHOP SR, TERRELL

1 P GAS TECHNICIAN

ARKANSAS BUREAU OF STANDARDS

ROUTE $B O X 357$

RUSSELLVILLE. AR 72801

BURNS RANDY

MM LABORATORY TECHN ICI AN

ARKANSAS BUREAU OF STANDARDS

4608 WEST 61 ST ST

LITTLE ROCK: AR 32209

CHESSER CLEN

INVESTIGATIVE SUPERVISOR

ARKANSAS BUREAU OF STANDARDS

4608 HEST $61 S T$ ST

LITTLE ROCK: AR 72209

CUSHING KENNETH

INVESIIGATOR II I I I STANDARDS

4608 WEST 61 ST ST

$501371-1759$ AR 72209

FRAZIER LOYD

ARKANSAS BUREAU OF STANDARDS

2640 COUNTRY WAY

FAYETJEVILLE. AR 72703

FRAZIER, RDGER

INVESTIGAT IVE SECTION

ARKANSAS BUREAU OF STANDARDS

211 PRINCETON

BENTON: AR 72015

$501371-1759$

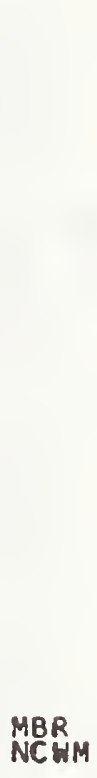

MBR

NCWM

MBR

NCWM

MBR

NCWM

MBR

NCWM

MBR
NCWM

MBR

NCWM

MBR

NCGM 
HARRINGTON: MARVIN L

INVESTIGATOR II

RT 3 BOX 140

GREENBRIER, AR 72058

HILE. MIKE

ASS SSTANT PIRECTOR

4608 HEST $61 S T$ ST

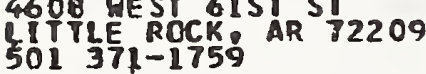

HINOSMAN, SAM F

DIRECTOR BUREAU OF STANDARDS

4608 WIST ST

LITTLE ROCK AR 72209

$501371-1736$

KARLISH DAVIDO

CHEMIST SUPERVISOR

CARKANSAS BUREAU OF STANDARDS

LITTLE ROCK: AR 72209

501 371-1759

KIRSPEL JR CHARLES

REAU OF STANDARDS

4608 WEST $6 I S T$ ST

LITLE RCCK: AR 72209

$501371-1759$

KIRSPEL SR CHARLES B

W. E INVESTIGATOR I

50.9 WEST A AVENUE

N LITTLE AR 72116

$501753-4751$

LASTER A ABE A

ARKANSAS BUREAU WTS \& MEAS

CLARKVILLE 35 AR 72830

MCCOSKEY JAMES

INVESTI GATOR I

ARKANSAS BUREAU OF STANDARDS

$250 \mathrm{C}$ PHILLIPS ST

PINE BLUFF AR 71603
$501371-1759$

PIERCE\& MARCIE

ADHINIS TRATIVE ASST II II ATARDS

4608 HE ST 61 ST ST

LITLE ROCK: AR 72209

$501371-1759$

PRINCE, LEON B

WE M INVESTIGATOR I ARKANSAS BUREAU

HOPE, AR 71801

$501371-1759$

SHELTON WO

INVESTIGATOR I

ARKANSAS BUREAU OF STANDARDS

815 CEDAR LANE

$501371-1759$ AR 72903

SULLIVANT: BILLY W

LABDRATCRY SUPERYISOR

4608 WEST 61 ST

LITTLE RCCK: AR 72209

MBR

MBR

MBR

MBR

MBR

MBR
NCWM

NCWM

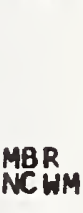

NCWM

MBR

NCHM

MBR

$501371-1759$

MBR

NCWM

MBR
NCWM
SUAMERVILLE, IIM

INVESTIGATCR II I OF STANDARDS

4608 AREST $61 S T$ ST

LITLE ROCK: AR 72209

$501371-1759$

THOMAS. KEN

INVESTIGATOR IOR OL OF STANDARDS 4600 HEST EIST ST

LITTLE ROCK. AR 72209

$501371-1759$

WEAVER, JAMES

INVESTIGATOR II

ARKANSASAUREAU OF STANDARDS

4608 HEST $61 S T$ ST

LITTE ROCK AR 72209

$501371-1759$

WESTMORELAND. ARCH

INVEANSAS BUREAU OF STANOAROS

RTKAN

SIDNEY, AR 72577

$501371-1759$

WHEELER: HARLIN

INVE ST I GAT CR I

ARKANSAS BUREAU OF STANOARDS

RT 8 BOX 278 AA

PARAGOULD
501 AR $7245 \mathrm{C}$

CAL IFORNIA

STATE

AGNEW. $M$

SPECIAL INVESTIGATCR I

CA DIV OF MEASUREMENT STANDARD

169 E L IBERTY AVE

ANAHEIHOCA 92801

$714680-7896$

ALVELAIS FRANK

QUANTITY CONTROL SPEC I

CA DIV OF MEASUREMENT STANDARD

169 E LIBERTY AVE

ANAHEIM. CA 92801

$714680-7896$

BFEBE, CHARLES

PROGRAM SUPERV ISOR

CA DIV OF MEASUREMENT STANDARD 8500 FRUITRIDGE RD

SACRAMENTO. CA 95826

$916366-5119$

BLDCH BARBARA J

ASSISTANT CHIEF

CA DIV OF MEASUREMENT STANDARD

8500 FRUITRIDGE RO

SACRAMENTO CA 95826

916 366-5119

BRANUM, PAUL

MANAGER

CA DIV OF MEASUREMENT STANDARD 8500 FRUITRIDGE RD

SACRAMENTO: CA 95826

916 366-5119

BRUSH JACK SR SPECIAL INVESTIGATOR

CA DIV OF MEASUREMENT STANDARD 3609 BECHELLI LANE/RDOM E REDDING. CA 96002

916 246-6201 
CAMCZZI E WALTER A

CA CIV OF MEASUREMENT STANDARD 1500 FRUITRIDGE RD

SACR AMENTD 9 CA 95826

CHIESA, LAURENE

SR SPEC IALL INVESTIGATOR

CA DIV CF MEASUREMENT STANDARD 2550 MARIPOSA ST ROOM 3044

FRESNO $C_{2} 93721$

CLEARY, MICHAEL

SPECIAL INVESTIGATOR

CA DIV OF MEASUREMENT STANDARD

8500 FRUITRIDGE RD

SACRAMENTO CA 95826

CLINEZ JOHN

SR SPEC IAL INVESTIGATOR

CA DIV OF MEASUREMENT STANDARD 255C MARIPOSA ST ROOM 3044

FRESNO CA 93721

$209445-5403$

COOK, STEVEN E

W E TECHNICIAN II
CA DIV OF MEASUREMENT STANDARD
S5OO FRUITRIDGE RD

SACRAMENTO CA 95826

$916366-5119$

CORRICK, BERT G

PROGRAM SUPERVISOR

CA DIV OF MEASUREMENT STANDARD

MaR

8500 FRUITR IDG

SACRAMENTO CA 95826

$916366-5119$

DELPERDANG MARIANNE CA DIV CF HEASURE MENT ST ANDARD

169 E L IBERTY AVE

ANAHEIM CA 92801

$714680-7896$

DOYLE, ROBERT S

CA MIV TECHNICIAN III II STANDARD

8500 FRUITRIDGE RO

SACRAMENTO CA 95826

916 366-5119

DRURY JEANIE

SR SPECIAL INYESTIGATOR

CA DIV OF MEASURE HENT STANDARD

169 E L IBER TY AVE

ANAHEIM:CAA 92801

DUNLAP DAVID

SR SPEC IAL I NYESTIGATOR

CA DIV OF MEASUREMENT STANDARD

ANAHEIM CA 92801

$714680-7896$

EHL Y Y ROBERT N

SR SPEC INYESTIGATOR

III JACKSON ST ROON 2002

OAKLAND. CA 94607

$415464-1221$

FLETCHER, THOMAS

WE IGHTS \& MEA SURES TECH III

CA DIV OF HEASUREMENT STANDARO

8500 FRUITRIDGE RO

SACRAMENTOP CA 95826
$916366-5119$
FLORES RONALD

CE E TECHNICIAN IL TIENT STANDARD 8500 FRUITRIDGE RD

SACRAMENTO CA 95826

916 $366-5119$

FRYDMAN KAREN

QUANTITY CONTROL SPECIALIST II CA DIV OF MEASUREMENT STANDARO 3609 BECHELLI LANE/ROOM E

REDDING. CA 96002

$916246-6201$

GANDSEY, WILLIAM N

SUPERVIS ING SPEC INVESTIGATOR CA DIV OF MEASUREMENT STANDARD 169 E LIBERTY AVE

ANAHEIM, CA 92801

$714680-7896$

GARCIA. SYLVIA

JUNIOR CHEMIST

CA DIV OF MEASUREMENT STANDARD

169 E LIBERTY AVE

ANAHE IM. CA 92801

$714680-7896$

GODINA, GREGORY

SR SPECI AL INVESTI GATOR

CA DIV OF MEASUREMENT STANDARD 169 E LIBERTY AVE

ANAHEIM. CA 92801

$714680-7896$

GONZALES: MANUEL

BRANCH CHIEF

CA DIV OF MEASUREMENT STANDARD

8500 FRUITRIDGE RD

SACRAMENTO 16 CA 95826

GORDOND PEARL

CA DIV OF MEASUREMENT STANDARD 8500 FRUITRIDGE RD

SACRAMENTO. CA 95826

916 366-5119

GOHER CHERYL

G G M TECHNICIAN III

CA DIV OF MEASUREMENT STANDARD 8500 FRU ITRIDGE RD

SACRAMENTO CA 95826

GUENSLER • DARRELL A

CHIEF

CAL IF DIV OF MEASUREMENT STD

8500 FRUITRIDGE RD

SACRAMENTO 9 CA 95826

HARR IS, MARSH

CA M TECHNICIAN III II STANDARD III JACKSON ST ROCM 2002 DAKLAND. CA 94607

$415464-1221$

HERBERT' AL

CUPERVISING CHEMIST Q500 FRUITRIDGE ROAD

SACRAMENTO CA 95282

$916366-5119$

HERNANDEZ, MAR IA

SPECIAL INVESTIGATOR

CA DIV OF MEASUREMENT STANDARD

169 E LIBERTY AVE

ANAHEIM:CA 92801

$714680-7896$
MBR

NCWM

MBR

NCWM

MBR

NCWM 
HOPKINS JAMES

W. \& TECHNICIAN II

CA DIV OF MEASUREMENT STANDARD 2550 MARIPOSA ST ROCM 3044 $209445-5403$

JENKINS E EARL

WEIGHTS \& MEASURES TECH III

CA DIV CF MEA SUREMENT STANDARO $850 C$ FRUITRIDGE RD

SACRAMENTO, CA 95826

\section{JOHANNES DENNIS}

DI E MF MEHNICIAN II STANDAROS

8500 FRUITRIDGE R.OAD

SACRAMENTD CA 95826

KELLY, MICHAEL

W E M TECHNICIAN III

CA CIV CF MEASUREMENT STANDARD

360 S BECHELLI LANE/ROOM E

REDDING . CA 96002

$916246-6201$

KINGREN。 DONNA L

SPECIAL INVESTIGATOR I

CA DIV CF MEASUREMENT STANDARD

$850 C$ FRUITRIDGE RD

SACRAMENTO CA 95826
$916366-5119$

LABOSSIERE PIERRE

W \& M TECHNICIAN II

CA DIV CF NEASUREMENT STANDARD

SACRAMENTO CA 95826

\section{LAKE, KEN}

PROGRAM SUPERVISOR

CA DIV OF MEA SUREMENT STANDARD 8500 FRUITRIDGE RD

SACRAMENTO CA 95826

LAZIER, DAVID

SUPERVISING SPEC INVESTIGATOR

CA DIV OF MEASUREMENT STANDARD 8500 FRUITRIDGE RD

SACRAMENTO CA 95826

LEWIS: BERRIE L

QUANTITY CONTROL SPECI ALIST II

111 JACKSON ST ROOM 2002

$415464-1221$

LUSE NEY J

QUANTITY CONTROL SPECIALIST II

CA DIV CF MEASUREMENT STANDARD

850 C FRUITR IDGE RD

SACRAMENTO CA 95826

LYCN CYNTHIA

PETRÓl UM LAB SPECIALIST

85 C MTANDARD

$850 C$ FRUITRIDGE RD

SACRAMENTO CA 95826

MALLOY RAYMOND

CA DIV OCHNICIAN II

8500 FRUITRIDGE RD

SACRAMENTO CA 95826
916 366-5119
MBR

NCHM

$M B R$

NCWM

MARTIN. EARL E

QUANTITY CONTROL SPECIALIST II CA DIV OF MEASUREMEAT STANDARO 8500 FRUITRIDGE RD

SACRAMENTO. CA 95826

$916366-5119$

MICHEL TOM

WEIGHTS \& MEASURES IECH III

CA DIV OF MEA SUREYENT STANDARD 8500 FRUITRIDGE RD

SACRAMENTO CA 95826

MOUGH, JOHN

PETROLEUM PRODUCTS CHEMIST

CA DIV OF MEASUREMENT STANDARD

169 E L I BERTY AVE

ANAH!EIH. CA 9280 I

$714680-7896$

MURRAY, JOHN

W \& $M$ TECHNICIAN III

CA DIV DF MEASUREHENT STANDARD

169 E L IBERTY AVE

ANAHEIM, CA 92801

$714680-7896$

NICHOLS MICHAEL

W \& M TECHNICIAN II

CA DIV OF MEASUREMENT STANDARO 8500 FRUITRIDGE RD

SACRAMENTO CA 95826

PIFER , SANDRA

NE M TECHNICIAN II

CA DIY OF MEASUREMENT STANDARD

\& 500 FRUITRIDGE RO

SACRAMENTO CA 95826
$916366-519^{2}$

PITTA-YRI ZZONT JUAN

SPECIAL INVESTIGATOR I

CA DIV OF MEASUREMENT STANDARD

1111 JACKSON ST ROCM 2002

OAKLAND. CA 94607

415 464-1226

PQROSHIN, CARDLE

PETROLEUM LAB SPECIALIST

CA DIV OF MEASUREMEIIT STANDARD 8500 FRUTIRIDGE RD

SACRAMENTOO CA 95826

$916366-5119$

RETTELA。 GERALD P

SENIOR SPECIAL INVESTIGATOR

CA DIV OF MEASUREMENT STANDARD

$169 \mathrm{E}$ LIBERTY AVE

ANAHEIMV CA 92801

$714680-7896$

ROSSSOTTCM, THOMAS

WEIGHTS $\varepsilon$ MEASURES TECH II

CA DIV OF MEASUREMENT STANDARO 8500 FRUITRIDGE RD

SACRAMENTO CA 95826
916 366-51

ROTHLEDER, JOSEPH

PRINCIPAL STATE METRCLCGIST

CA DIV OF MEASUREMENT STANDARD 8500 FRUITRIDGE ROAD

SACRAMENTO CA 95826
$916366-51.9$

SALING。 MIKE

PROGRAM SUPERVISOR

CA DIV OF MEASUREMENT STANDARD

8500 FRUITRIDGE RD

SACP.AMENTO. CA 95826

$916366-5119$ 
SCHLEICHER ARTHUR

H E M TECHNICIAN II NT STANDARD 8500 FRUITRIDGE RD

SACRAMENTO CA 95826

916 366-51 is

SEFCIK, JOHN

CA DIV CF MEASUREMENT STANDARD

169 E IBERTY AVE

ANAHEIM, CA 92801

$714680-7896$

SMITH, CLIFTON E

BRANCH CHIEF

CA DIV OF MEASUREMENT STANDARD

8500 FRUITRIDGE RD

SACRAMENTO CA 95826

916 366-5119

SMITH F RICHARD

SR SPEC INVESTIGATOR

CA DIV OF MEASUREMENT STANDARD 8500 FRUITRIDGE RD

SACRAMENTD CA 95826

916 366-5119

SNYCER, W JAMES

SR SPEC IAL INVESTIGATOR

CA CIV OF MEASUREMENT STANDARD

8500 FRUITRIDGE RD

SACRAMENTO CA 95826

916 366-5119

SPARKS, NORMAN B

SUPERVI SING SPEC INVESTIGATOR

CA CIV CF MEASUREMENT STANDARD 8500 FRUITRIDGE RD

SACRAMENTO CA 95826

916 366-5119

SPELL. EARLE

H E TECHNICIAN I

CA DIV OF MEASUREMENT STANDARD 8500 FRUITRIDGE RD

SACRAMENTQ CA 95826
$916366-5119$

STEMART, CHARLES

QUANTITY CONTROL SPECIALIST II CA DIV CF MEA SUREMENT STANDARD 2550 MARIPOSA ST ROCM 3044

$209445-5403$

TAMAI SALLY

STAFF SERVICES ANALYST

CA DIV OF MEA SUREMENT STANDARD

8500 FRUITRIDGE RD

SACRAMENTO CA 95826

916 366-5119

THUNER, JEFFREY

CA DIV OF MEASUREMENT STANOARD

169 E LISERTY AVE

ANAHEIM. CA 92801

$714680-7896$

TOLLEFSCN. JAMES L

W E M TECHNICIAN III I OF MEASUREMENT STANDARD

MBR

8500 FRUITRIDGE RD

SACRAMENTD CA 95826

$916366-5119$

UYEHARA \& RICHARD

JUNIOR CHEMIST

CA CIV OF MEASUREMENT STANDARD

169 E IBERTY AYE

$714680-7896$
MBR

NCHM

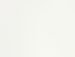

WARTMAN JAMES C

SR SPECIAL INVESTIGATOR

CA DIV OF MEASURENENT STANDARD 2550 MARIPOSA ST RCOM 3044 FRESNOE CA 93721

$209445-5403$

WORCESTER, FLOYD $B$

CA DIV OF MEASUREMENT STANDARD 8500 FRU ITRIDGE RD

SACRAMENTO. CA 95282

$916366-5119$

YOUNG RUTH

SR SPEC INVESTIGATCR

CA DIV OF MEASUREMENT STANDARD

8500 FRUITRIDGE RD

SACRAMENTO, CA 95826

$916366-5119$

ALAMEDA COUNTY

AYRES JOSEPH J

SUPERVISING IN SPECTCR

DEPT OF WE IGHTS \& MEASURES

333 FIFTH ST

OAKLAND. CA 94607

BUSCH. PAUL A

INSPECTOR

DEPT OF WEIGHTS 6 MEASURES

333 FIFTH ST

OAKLAND, CA 94607

$415874-6736$

CHAPMAN LEHIS R

SENIOR INSPECTOR

DEPT OF WEIGHTS E MEASURES

333 STH ST

OAKLAND. CA 94607

$415874-6736$

HASEMEYER, RONALD C

INSPECTOR

OEPT OF WEIGGHS \& MEASURES

333 STH ST

OAKLAND. CA 94607

$415874-6736$

JENSEN. FR ANK E

WEIGHTS \& MEASURES INSPECTOR 333 5TH ST

OAKLAND. CA 94607

$415874-6736$

JOROAN, ESTELLA M

DNEPT DF WEIGHTS \& MEASURES

333 FIFTH ST

OAKLAND. CA 94607

$415874-6736$

MANGONON. ARTHUR L

WEIGHTS E MEASURES INSPECTOR 333 STH ST

OAKLAND. CA 94607

$415874-6736$

NICHOLS. PATRICK E

SEALER

DEPT OF WEIGHTS AND MEASURES

MBR

333 FIFTH ST

OAKLAND。CA 94607

415 874-6736

OLEARY MERRY L

INSPECTOR

DEPT DF WEIGHTS \& MEASURES

333 FIFTH ST

OAKLAND. CA 94607

$415874-6736$ 


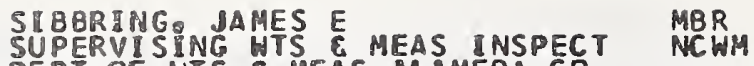
DEPT OF UTS 6 MEAS ALAMEDA CD 333 STH ST

OAKLAND. CA 94607

$415874-6736$

TREVELPIECE CHARLES E

SEAIIOR INSPECTOR

333 PIFTH ST

DAKLAND? CA 94607

$415874-6736$

\section{AMADOR COUNTY}

FARNHAM RCBERT L

DEPT OF WEIGHTS \& MEASURES

SUTER HILL/108 COURT ST

JACKSON. CA 95642

$209223-6487$

THOMPSOA DAYID A

AMADOR COUNTY

SUT YER HILL 108 COURT ST

AACKSON CA 95642

$209223-6481$

UMINO. ROBERT

DEPUTY DIRECTOR

DEPT OF WEIGHTS \& MEASURES

SUTTER HILL/IOS COURT ST

JACKSON。 CA 95642

$209223-6487$

\section{BUTTE COUNTY}

BANDY JOE E

DEPT OF WEIGHTS \& NEASURES

316 NEL SON AVEJPO BCX 1229

ORCVILE $C A 95965$

$916538-7381$

TRECK JAMES C

ONSPECTIOR IOF WEIGHS \& MEASURES

196 MEM:CR IAL HAY

CHICO CA 95926

$916891-2756$

LOMSARO HAROLD H

DEPT OF WEIGHTS \& MEASURES

196 MEMORIAL WAY

CHICO CA 959
$916891-2756$

STRCM, RICHARD W

DEPUTY DIRECTOR WEIGHTS $E$ MEAS

CALIFORNIA WUIGHTS \& MEASURES

196 MEMORIAL WAY

CHICD. $C A 95926$

$916891-2756$

\section{CALA VERAS COUNTY}

HOWARD JEARL D

DERECTORR

OEPT OF HEIGHTS \& MEASURES

GOYERNHENT CTR EL DORADO RD

SAN ANDREAS CA 95249

$209754-4142$
KERSTAN: KARL

INSPECTOR III

DEPT OF WE IGHTS $\&$ MEASURES

GOV CENTER/EL DORACO RD

SAN ANDREAS. CA 95249

$209754-4142$

NORFOLK: DOUGLAS

INSPECTOR III

DEPT OF WEIGHTS \& MEASURES

GOV CENTER/EL DORACC RD

SAN ANDREAS. CA 95249

$209754-4142$

\section{COLUSA COUNTY}

AZEVEDO MARY ANN

DEPT OF WEIGHTS 6 MEASURES

751 FREMONT ST

COLUSA CA 95932

BRDWN: DARYL ${ }^{B}$
DEPUTY SEALER

OEPT OF WEIGHTS E MEASURES

751 FREMONT ST

COLUSA CA 95932

$916458-5367$

CAPEHART: BOB L

INSPECTOR III

751 FREMONT ST 20

$916458-5867$

DISNEY JR, WILBER C

SEALER

DEPT OF WEIGHTS $\&$ MEASURES

100 SUNRISE BLVD \#F/POB 1029

$916458-5867$

RICHTER JON I

INSPECTOR IIITS \& MEASURES
DEPT OF WEIGHTS \& MENONT ST

COLUSA. CA 95932

$916458-5867$

TAUSCHER - JOSEPH C

INSPECTOR II

DEPT OF WEIGHTS \& MEASURES

751 FREMONT ST

COLUSA: CA 9

CONTEA COSTA COUNTY

DEFREMERY JOHN ${ }^{H}$ ' MEASURES

DIRECTOR WEIGHTS E MEATUENT OF AGR I CULTURE

161 JOHN GLENN DR

CONCORD. CA 94520

$415671-4250$

ESPEJOP CRIS

DEPT DF WE IGHTS 6 MEASURES

161 JOHN GLENN DR/BUCHANAN AP CONCORD. CA 94520

$415671-4250$

FORSEY INSPECTOR I NDA

DEPT OF WEIGHTS $\&$ MEASURES

161 JOHN GLENN DR/RUCHANAN AP

CONCORD. CA 94520

$415671-4250$ 
GUINN. DREXEL B

CHIEF DEPUTY SEALER

DEPT DF WEIGHTS E MEASURES

161 JOHN GLENN DR/BUCHANAN AP CONCORD. CA 94520

$415671-4250$

JODAR, BYRON

SUPERVI SING INSPECTOR

DEPT OF WEIGHTS \& MEASURES

161 JOHN GLENN DR/BUCHANAN AP CONCORO CA 94520

$415671-4250$

MADDUX CHARLES

SUPERVI SING INSPECTOR

DEPT OF WEIGHTS \& MEASURES

161 JOHN GLENN DR/BUCHANAN AP CONCORD CA $\$ 4520$

\section{ROBINSON, KENNETH}

HEAVY CAPACITY INSP ECTOR

DEPT OF HEIGHTS \& MEASURES

161 JOHN GLENN OR/BUCHANAN AP CONCORD. CA 94520

$415671-4250$

WALLACE DAVIO

INSPECTCR I HTS \& MEASURES

161 JOHN GLENN DR/BUCHANAN AP CONCORO. CA 94520

$415671-4250$

EL NORTE COUNTY

ANDERSON, GLENN E

INSPECTOR

DEPT DF WEIGHTS 6 MEASURES

2650 WASHINGION BL VO

CRESCENT CITY. CA 95531

$707464-2716$

BUSH. DONALD W

SEALER

DEPT OF HEIGHTS \& MEASURES

2650 WASHINGTON BLVD

CRE SCENT CITY, CA 95531

BUTLER ROBERT E

INSPECTCR

DEPT OF WEIGHTS \& MEASURES

265 C WASHINGTON BLVD

CRESCENT CITY. CA 95531

$707464-2716$

\section{EL DORADO COUNTY}

BOLTON MARJORIE

INSPECTOR

OEPT OF WEIGHTS \& MEASURES

31 FAIR LANE

PLACERVILLE CA 95667

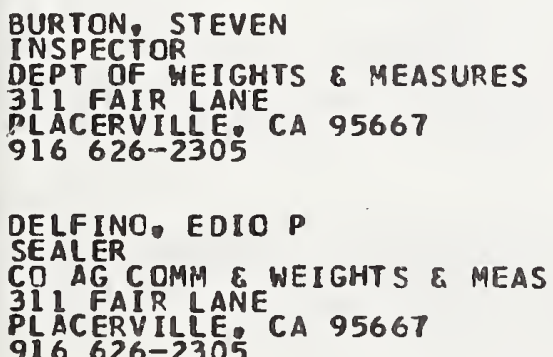

BURTON, STEVEN

INSPECTOR OF WEIGHTS \& MEASURES

311 FAIR LANE

PLÁCERVILLE.CA 95667

$916626-2305$

DELFINO. EDIO P

SEALER

CO AG COMM \& WEIGHTS \& MEAS

311 FAIR LANE

PLACERYILLE CA 95667

LOCHER CARL

DEPT OF HE IGHTS \& MEASURES 311 FAIR LANE

PLACERVILLE, CA 95667

$916626-2305$

STEWART ROBERT

INSPECTOR

DEPT OF WEIGHTS \& MEASURES 311 FAIR LANE

PLAC ERVILLE CA 95687
916 626-2305

\section{FRESNO COUNTY}

BLACK. ALFRED L

INSPECTOR III OFT \& MEASURES DEP OF WEIGHTS

$209488-3525$

BOREC: ROBERT

INSPECTOR

DEPT OF WE IGHTS \& MEASURES

4535 E HAMILTON

$209488-3525$

CERVANTES, LORI

INSPECTOR II

DEPT OF WEIGHTS 8 MEASURES

4535 E HAMILTON

FRESNO. CA 93702

$209488-3525$

FIELDS DONALO W

SENIOR INSPECTOR

4535 E HAMILTON

FRESNO. CA 93702

$209488-3601$

INSALACO, COSMO $C$

AGR IC COMMISSIONER/ SEALER

FRESNO COUNTY

1730 SOUTH MAPLE

FRESNO. CA 93702

$209488-3510$

LYMAN. OUANE

SENIOR INSPECTOR

DEPT OF WEIGHTS \& MEASURES

4535 E HAM ILTON

FRESNO CA 93702

$209488-3525$

NYBERG. THOMAS

INSPECTOR IIITS \& MEASURES

4535 E HAMILTON

FRESNO. CA 93702

$209488-3602$

SAUM. BRET I

INSPEC TOR II

DEPT OF WEIGHTS \& MEASURES

4535 E HAMILTON

FRESND。CA 93702

$209488-3603$

WALL DUANE

INSPECTOR III

DEPT OF WEIGHTS 6 MEASURES

4535 E HAM ILTON

FRESNO. CA 93702

$209488-3525$
MBR 


\section{GLENN COUNTY}

DUCKWORIH JR WILLIAM R

ASSISTANT SEALER

DEPT OF HEIGHTS \& MEASURES $720 \mathrm{~N}$ COLUSA ST/PO BOX 351 916 L $935-4651$

EHING KENNETH

INSPECTCR III

$720 \mathrm{~N}$ COLUSA ST/PO BOX 35

WILLCWS. CA 95988

$916934-4651$

MCINTYRE, JOE

DEPT OF HEIGHTS \& MEASURES

$720 \mathrm{~N}$ COLUSA ST/PO BOX 351

ILLOWS. CA 95988

$916934-4651$

PALMER, NOLA

INSPECTOR III

DEPT OF WEIGHTS \& MEASURES

720 N COLUSA ST/PO BOX 351 916 L 935 S CA

ROMANO. ED

DEALER OF WEIGHTS \& MEASURES P O BOX 351

916 LOHS. CA 95988

SIMPSON ERNE ST

INSPECTOR III

DE PT OF HE IGHTS $E$ MEASURES

HILLWS. CA 95988

$916934-4651$

STENLUND, GEORGE

INSPECTOR III

DEPT OF HE IGHTS \& MEASURES

$720 \mathrm{~N}$ COLUSA ST/PO BOX 351

916 H34-4651 95988

TOLLEY JOHN F B

INSPEC TOR III

DEPT OF WEIGHTS \& MEASURES

$720 \mathrm{~N}$ CCLUSA ST/PO BOX 351

WILLOWS. CA 95988

$916934-4651$

HUMBOLDT COUNTY

DRELRY RICHARD

SUPERVISING INSPECTOR

DEPT OF WEIGHTS \& MEASURES

727 CEDAR ST

GAREERVILLE: CA 95440

FALKENSTROM, JOHN

SEALER

DEPT OF WEIGHTS $\&$ MEASURES

$563 \mathrm{C}$ SOUTH BROADHAY

EUREKA: CA 95502

$707445-7223$

HAGGARD PETER

INEPTECTOR OII WEIGHS \& MEASURES

563 C S BROADWAY

EUREKA. CA 95502

707 445- 7223
HOLZBERGER PAUL

INSPECTOR II I

DEPT OF WEIGHTS \& MEASURES

5630 S BRDADWAY

EUREKA. CA 95502

707 445-7223

HUNT JOE E

DEPT OF WEIGHTS \& MEASURES

5630 S BROADWAY

EUREKÄ. CA 95502

$707445-7223$

SIEVERT, JOHN

DEPT OF WE IGHTS \& MEASURES

5630 S BROADHAY

EUREKA, CA 95502

$707445-7223$

SIMAS. WARREN

INSPECTOR II

DEPT OF WEIGHTS \& MEASURES

5630 S BRDADWAY

EUREKA. CA 95502

707 445-7223

SPADONI R ICHARD

INSPECTOR III

DEPT OF WEIGHTS \& MEASURES

5630 S BROADWAY

EUREKA. CA 95502

$707445-7223$

SPALLINO, JAMES

ASSISTANT SEALER MEASURES

5630 S BROADWAY

EUREKA. CA 95502

$707445-7223$

IMPER IAL COUNTY

BYRD。 DARR ELL E

DEPT OF WE IGHTS $\&$ MEASURES

150 SOUTH 9TH ST

EL CENTRO CA 92243

FINNELL CLAUDE $M$

COUNTY HEIGHTS \& MEASURES DEPT

150 SOUTH NINTH ST

EL CENTRO CA 922243

MODRE. ROBERT C

SUPERVIS ING DEPUTY SEALER

DEPT OF WE IGHTS \& MEASURES

150 SOUTH 9TH ST

EL CENTRD, CA 92243

$619339-4314$

\section{INYO AND MONO COUNTY}

MILOVICH: GEORGE L

INSPECTOR

DEPT OF WEIGHTS \& MEASURES

COUNTY SVC BLD/207 W SOUTH ST

BISHOP. CA 93514

$714873-5891$

MUSE DONALD R

OIRECTOR OF WEIGHTS \& MEASURES

INYO \& MONO COUNTIES

BISHOP. CA 93514

$714873-5891$ 
BROCKS. JOSEPH $V$

SENIOR DEPUTY

DEPT OF WEIGHTS $\&$ MEASURES

11 E EAL I FORNIA A VE

$805861-2418$

CERYANTES SCCORRO

DEPT OF WEIGHTS $\&$ MEASURES

II 16 E CAL I FORNIA AVE

BAKKER SF IELD
$805861-2418$

OYAS, DAVID

INSPECTCR

DEPT OF WEIGHTS 6 MEASURES

1116 E CAL IFORNIA AVE

$805861-2418$

HARRIESR JOHN OO

ASST DIR WEIGHTS \& MEA SURES

116 E CAL IFORNI A AVE

BÁKERSFIELD. CA 93307

$805861-2418$

LOWE, VERNON L

DIRECTOR OF WEIGHTS $\&$ MEASURES

MBR

DEPT OF WEIGHTS $\&$ MEASURES

II I6 E CALIFORNIA AVE

BAKERSF IELD
$805861-2418$

MULLICAN RICHARD L

SENIOR CEPUTY

DEP OF HEIGHTS \& MEASURES

1116 E CALIFORNIA AVE

BARERSFIELD, CA 93307

$805861-2418$

PHILLIPS, SENDE

DEPT OF WEIGHTS \& MEASURES

1116 E CAL I FORNIA A VE

BAKERSF I ELD.

WELLS: ROYAL $C$

OEPT OF WEIGHTS $\&$ MEASURES

BAKERSFIELD CA 93307

$805861-2418$

WHELDEN JACK $R$

DEPUTY SEALER

DEPT OF WEIGHTS \& MEASURES

1116 E CALIFORNIA A VE

BAKERSF IELD, CA $933 \mathrm{CT}$

$805861-2418$

INGS COUNTY

BERRY PAUL

AG E STANDARDS INSPECTCR II

DEPT OF WEIGHTS \& MEASURE 280 CAMPUS DR/GOV CENTER

HANFDRD 2 CA 93230
$209582-3211 \times 2830$

BOOKOUT LLLOYD W

DEPT OF WEIGHTS \& MEASURES

280 CAMPUS DR/GOV CENTER

HANFORD. CA 93230

$209582-3211 \times 2830$
BRAUTIGAM LEWIS H

DEPUTY SEALER

DEPT OF HEIGHTS 6 MEASURES

280 CAMPUS DR/GOV CENTER

HANFORD. CA 93230

$209582-3211 \times 2830$

BRAY - DENNIS

AG \& STANDARDS INSPECTOR III

DEPT OF WEIGHTS \& MEASURES

280 CAMPUS DR/GOV CENTER

HANFORD. CA 93230

COLVIN, STEPHANIE

AG \& STANDAROS INSPECTOR II

DEPT OF WEIGHTS \& MEASURES

280 CAMPUS DR/GOV CENTER

HANFORD. CA 93230

$209582-3211 \times 2830$

COSTA. BERNARO L

AG \& STANDARDS INSPECTOR III

DEPT DF WEIGHTS \& MEASURES

280 CAMPUS DR/GOV CENTER

HANFORO. CA 93230

$209582-3211 \times 2830$

OODD. JAMES L

AG \& STANDARDS INSPECTOR III

DEPT CF WEIGHTS \& MEASURES

280 CAMPUS DR/GOV CENTER

HANFORD. CA 93230

$209582-3211 \times 2830$

GIFFORD, CHARLES $M$

AG E STANOARDS INSPECTOR II

DEPT OF WEIGHTS $E$ MEASURES

280 CAMPUS DR/GOV CENTER

HANFORD. CA 93230

$209582-3211 \times 2830$

HANDLEY HUGH

KINGS COUNTY WEIGHTS E MEASURE

280 CAMPUS DR

HANFORD. CA 93230
$209582-3211 \times 2830$

HOPPER, MONTY H

DEPUTY SEALER

DEPT OF WE IGHTS $\&$ MEASURES

280 CAMPUS DR/GOVT CENTER

HANFORD. CA 93230

$209582-3211$

LEONI MICHAEL P

AG \& STANDARDS INSPECTOR III

DEPT DF WEIGHTS $\&$ MEASURES

280 CAMPUS DR/GOV CENTER

HANFORD. CA 93230

$209582-3211 \times 2830$

NICHOLSON, ODIS A

DEPUTY SEALER

DEPT OF WEIGHTS \& MEASURES

280 CAMPUS DR/GOV CENTER

HANFORD. CA 93230

$209582-3211 \times 2830$

REEVES, DIANE

AG E STANOARDS INSPECTOR II

DEPT OF WE IGHTS $C$ MEASURES

280 CAMPUS DR/GOV CENTER

HANFORD. CA 93230

REYMANN STEPHEN W

AG \& STANDARDS INSPECTOR III

DEPT OF WEIGHTS \& MEASURES

280 CAMPUS DR/GOV CENTER

HANFORD. CA 93230

$209582-3211 \times 2830$ 
RUSH, EOHIN

NDARDS INSPECTCR III

DEPT OF WEIGHTS E MEASURES

HANFORD. CA 93230

$209582-3211 \times 2830$

TOS LELAND $P$

DEPÜTY SEALER

DEPT OF WEIGHTS \& MEASURES

280 CAMPUS DR/GOV CENTER

HANFORD. CA 93230

$209582-3211 \times 2830$

UMINO, ROBERT

AG E STANDARDS INSPECTOR III

DEPT OF HE IGHTS EE MEASURES

HANFORD. CA 93230

$209582-3211 \times 2830$

\section{LAKE COUNTY}

JOHNSON RCANOICE L
ASST DIRECTOR OF WTS E MEAS
LAKE COUNTY DEPT WTS 6 MEAS

MBR.

883 LAKEPORT BL VO

$70726.3-2271$

MORSE, CHARLES

INSPECTCR

LAKE COUNTY OEPT WTS $\&$ MEAS

883 LAKEPORT BLVD

LAKEPOR T2 CA

NELSON CHARLES

LAKE COUNTY DEPT HTS \& MEAS

883 LAK EPORT BLVD

LAKEPORT 707 CA

TOMKINS EOWARD D

DIRECTOR OF WEIGHTS 6 MEASURES

LAKE COUNTY DEPT OF AGRIC

MBR

883 LAREPORT BLYO

$707263-2271$

\section{LASSEN COUNTY}

EYANSO FREORIC ICLISIONER SEALER

LASSEN COUNTY

175 RUS SELL AVE

SUSANVILLE CA 96130
$916257-83{ }^{2} \times 110$

HAWSE DON L

DEPT OF MEIGHTS \& MEASURES

175 RUS SELL AYE

SUSANVILLE CA 96130

SOMERVILLE ROBERT

DEPT OF HEIGHTS \& MEASURES

175 RUSSELL AVE

SUSANVILE CA 96130

\section{LOS ANGELES COUNTY}

BALLEKEN M.

DEPT OF WEIGHTS \& MEASURES

IIO12 GARFIELD AYE

SOU TH GATE CA 90280
BERUMEN ROBERT J

INSPECTOR IIIHTS G MEASURES

11012 GARF IELD AVE

$213940-8922$ CA 90280

8RAOY GEORGE

INSPECTOR I

DEPT OF WE IGHTS 6 MEASURES

11012 GARF IELD AVE

SOUTH GATE CA 90280

213 940-8922

BRITO, DAN

INSPECTOR I

DEPT OF WEIGHTS \& MEASURES

IIOL2 GARF IELD AVE

SOUTH GATE CA 90280

$213940-8922$

CHILOERS: WILLIAM E

INSPECTOR II

DEPT OF WE IGHTS 6 MEASURES

11012 GARF IELD AVE

$213940-8922$

DITON VICTORIA

INSPECTOR

DEPT DF HEIGHTS E MEASURES

11012 GARFIELD AVE

SOUTH GATE CA 90280

$213940-8922$

DOVE JR. ROBERT E

INSPECTOR II

DEPT OF HEIGHTS \& MEASURES

11012 GARF IELD AVE

SOUTH GATE CA 90280

213 940-8922

ELLIOTT: MARTIN

INSPECTÓR I

DEPT OF WE IGHTS \& MEASURES

11012 GARF IELO AVE

\$OUTH GATE CA 90280

ENGLER PAUL B

AGRIC COMAISSIDNER/SEALER

LOS ANGELES COUNTY

3400 LA MADERA AVE

EL MONTE CA 91732

$818575-5451$

GLUCK. NAHAN H

DEPUTY DIRECTOR

AGRICULTURE HEIGHTS 6 MEASURES

IOIZ GARF IELD AVE

SOUTH GATE CA 90280

213 940-8922

GONSER RICHARD L

DNSPECTIOR IIHTS 6 MEASURES

11012 GARF IELO AVE

SDUTH GATE CA 90280

213 940-8922

GUNDERSON. DALTON B

INSPECTOR II WTS 8 MEASURES

IIOL2 GARF IELD AṼE

SOUTH GATE. CA 90280

$213940-8922$

GUTIERREZ. FEL IPE

INSPECTOR

DE OF WEIGHTS 6 MEASURES

11012 GARFIELD AVE

SOUTH GATE CA 90280

$213940-8922$ 
MLLE GEORGE A

DT E M INSPECTICN

WEIGHTS $E$ MEASURES

OUTH

$13940-8922$

ENDERSON, RAY

XPY OF WEIGHTS \& MEASURES

IOLI2 GARFIELD AVE

JUTH GATE CA 90280

$13940-8922$

\section{OAR, THEODORE}

NSPECTIOR

1012 GARF IELD AV

DUTH GATE CA 90280

$13940-8922$

AMORA JOSEPH H

UP ERVI SING INSPECTOR

EPT OF hEIGHTS \& MEASURES

1012 GARF IELD AVE

OUTH GATE CA 90280

$13940-8922$

\section{OHNSON\& LORETTA}

EPT OF WEIGHTS $\&$ MEASURES

1012 GARF IELD AVE

OUTH GATE CA 90280

$13940-8922$

\section{NNOSTMAR, IRA}

IEPT OF WEIGHTS \& MEASURES

1012 GARF IELD AVE

:DUTH GATE:CA 90280

A TREILLE HAYNE

INSPEC TCR I IGHTS \& MEASURES

II 12 GARFIELD AVE

SOUTH GATE CA 90280

$213922-8916$

I VESEY PHILIP T

SUPERVI SING INSPECTOR

DEPT OF WEIGHTS E MEA SURES

SOUTH GATE CA 90280

$213940-8922$

MAHLER MARCUS J

INSPECTOR II

DEPY OF HEIGHTS \& MEASURES

IIOL2 GARFIELD AYE

$213940-8922$

MCDERMOTT $B P$

SUPERVI SING INSPECTOR

DEPT OF MEIGHTS \& MEASURES

11012 GARFIELD AVE

$213940-8922$ CA 90280

MONSON PAUL

DEPT OF WEIGHTS $\&$ MEASURES

SLO12 GARFIELD AVE

$213940-8922$

MOORE, HILLIAM

METROLOOGY TECHNICIAN I I

11012 GARFIELD AVE

SOUTH GATE CA 90280
$213940-8922$
NEALL Y HESLEY L

DEPT OF WEIGHTS \& MEASURES

11012 GARF IELD AVEE

SOUTH GATE CA 90280

213 940-8922

OLI VAN. AL FONSO

INSPECTOR I

DEPT OF WEIGHTS $\&$ MEASURES

11012 GARF I ELO AVE

$213940-8922$ CA 90280

PARRISHE CHARLES E

INSPECTOR II OFT 6 MEASURES

1012 GARF IELD AVE

SOUTH GATE 213 CA 90280

RAY ROBERT A

SUPERVISING INSPECTOR

DEPT OF WEIGHTS \& MEASURES

11012 GARF IELD AVE

SDUTH GATE CA 90280

$213940-8922$

ROYBAL, ROBERT L

INSPECTOR I

DEP 12 GAREIGHTS $\&$ MEASURES

11012 GARFIELD AVE

$213940-8922$

STUHR JR, ARTHUR $M$

CHIEF E M INSPECJION

DEP T OF WE IGHTS E MEASURES

11012 GARFIELD AYE

SIUTH GATE CA 90280

SUTHERLAND DARRELL $R$

INSPECTOR II HETS $\&$ MEASURES

11012 GARFIELD AVE

SOUTH GATE CA 90280

$213940-8922$

THOMPSON, JAMES $S$

INSPECTOR II

DEPT OF WEIGHTS \& MEASURES

11012 GARF IELD AVE

SOUTH GATE CA 90280

$213940-8922$

TURNER, COLLIS

CHIEFH E M INSPECTION

DEPT OF WE IGHTS E MEASURES

11012 GARF IELD AVE

SOUTH GATE CA 90280

WARE PHINEAS

INSPECTOR II

11012 GARFIELD AVE

SOUTH GATE CA 90280

213 940-8922

WILLIS, NORMAN J

INSPECTOR II

DEPT OF WEIGHTS $\&$ MEASURES

1110 WEST AVE J ROCM 4

LANCASTER. CA 93534

$805948-4615$

YAKUSHIJI- CHARLES T

INSPECTQR I

11012 GARF IELD AVE

SOUTH GATE:CA 90280

213 940-8922 
CRIPE DONALD O \& MEASURES MER MAOERA COUNTY

332 MADERA AVE MADERA, CA 93637

$209675-7876$

\section{KL UDJIAN, SETH}

DEPT OF WEIGHTS \& MEASURES

902 N GATEWAY DR

MADERA CA 93637

$209674-4641 \times 303$

\section{MARIN COUNTY}

8LACKWELH WILLIAH \& MEASURES DIRECTOR OF WEI

1450 LUCAS VALLEY RD

SAN RAFAEL CA 94903

$415 \quad 499-6349$

CARRINO, JOHN

ONSPECTOR II

MAR IN COUNTY CIVIC CTR RM 422

SAN RAFAEL CA 94903

$415499-6349$

FRAIRE PAUL A

INSPECTCR II

MARIN COUNTY CIVIC CTR RM 422

SAN RAFAEL CA 94903

\&15 499-6349

NELSON, ERLING R

DEPT DF WEIGHTS $\&$ MEASURES

DEPT OF WEIGHTS E MEASURES

SAN RAFAEL CA 94903

$415 \quad 499-63 \% 9$

SCHROCK, JACK W

INSPECTCR

DEPT OF WEIGHTS \& MEASURES

MARIN COUNTY CIVIC CTR RM 422

SAN RAFAEL CA 94903

$415499-63$.

MARIPOSA COUNTY

RILEY DAN

MARIPOSA COUNTY

3350 M ST 2

MERCED。CA 95348

MENDOCINO COUNTY

BENGSTON, DAYID

ASSISTANT DIRECTOR

OEPT OF WEIGMTS \& MEASURES

CDURTHOUSE

UR IAH CA 95482

DE GRASSI ROBERTO A

DIRECTOR

DEPT OF MEIGHTS $C$ REASURES

COURTHOUSE

UK IAH CA 95482
FARNSWORTH II KENNETH F

INSPECTOR II COURTHOUSE

UKI AH 407 CA 95482

LINCOLN\& STEVE

ONEPT OF WEIGHTS \& MEASURES

COURTHOUSE

UK I AH. CA 95482

T07 $463-4208$

\section{MERCED COUNTV}

LESLEET HILLIAM

DEPT OF WEIGHTS \& MEASURES

$2139 W$ WARORDBE AVE

MERCED. CA 95340

$209385-7431$

QUINN, MICHAEL J

INSPECTOR II

2139 WARDROBE AVE

MERCED: CA 953

RILEY, DAN

ASSISTANT DIRECTOR

DEPT OF WE IGHTS \& MEASURES

2139 WARDROBE AVE

MERCED. CA 95340

$209385-7431$

TANNER: MICHAEL J

DIRECTOR OF WEIGHTS \& MEASURES

MERCED COUNTY

2139 WEST WARDROBE

MERCED。 CA 95340

$209385-7431$

MODOC COUNTY

GFFENBACK, CLINTON B

SEALER OF WEIGHTS \& MEASURES

MODOC CDUNTY DEPT OF AG

202 H FOUR JH ST

ALTURAS CA 96101

$916233-2225$

SMITH KENNETH R

DEPUTY SEALER

DEPT OF WEIGHTS \& MEASURES

202 W FOURTH ST

ALTURAS CA \$101

$916233-2225$

\section{MONTEREY COUNTY}

GOODRICK JR. JOSEPH L

DIRECTOR

DEPT DF WEIGHTS \& MEASURES

120 ILGART WAY

SAL INAS. CA 93901

$408758-3876$

HUNTER JR, FRANCIS R

INSPECTOR II

DEPT OF WE IGHTS \& MEASURES

120 WILGART WAY

SAL INAS: CA 93901
$408758-3859$

PERSSON口 RUSSELL C

INSPECTOR III

DEPT OF WE IGTS \& MEASURES

120 WILGART WAY

SALINASS CA 93901

$408758-3859$ 
HAHN STEVEN R

INSPECTOR II

TAMAGNI TED

DEPT OF WEIGHTS $\&$ MEASURES

7292 SILVERADO TRAIL

NAPA CA 94558

$707944-8714$

VEVADA COUNTY

GUNDERSCN, ERIC R

INSPECTOR

DEPT OF WEIGHTS \& MEASURES

255 S AUBURN ST/VA MEM BLDG

GRASS VALLEY. CA 95.945

$916273-2648$

MILLS JOHN R NE VADA CCUNTY

255 SOUTH AUBURN ST

GRASS VALLEY.CA 95945

$916273-2648$

MBR

TAYLOR JOHN $V$ SEALER CF WEIGHTS \& MEASURES

255 S AUBURN ST 95945

916 273-264.8

WEBB, MARK

INSPECTCR

DEPT OF WEIGHTS \& MEASURES

255 S AUBURN ST / VA MEM BLDG GRASS VALLEY.CA 95945

$916273-2648$

\section{ORANGE COUNTY}

BENNETT MILTON D

DIST AGR ICULTURAL INSPECTOR

$1 O 1 C$ S HARBOR BLVO

ANAHEIM. CA 92805

$714774-0284$

CLODT JOHN C

DIST ÁGRICULTURAL INSPECTOR

DEPT OF WEIGHTS E MEASURES

IOIOS HARBOR BLVD

ANAHEIM. CA 92805

$714774-0284$

DANKER, BRI AN

DIST AGR I CULTURAL INSPECTOR

DEPT OF hEIGHTS \& MEASURES

1010 S HARBOR BLVD

ANAHEIM. CA 92805

$714774-0284$

FITCHEN, WILLIAM

SEALER DF HEIGHTS \& MEASURES

COUNTY OF ORANGE

1010 S HARBOR BLVO

ANAHEIM CA 92805

$714774-0284$

GROSSO ROBERT L

INSPECTCR II

DEPT OF WEIGHTS $\&$ MEASURES

1010 S HARBOR BLVD

ANAHEIM. CA 92805

$714774-0284$

MBR
DEPT OF WE IGHTS \& MEASURES

1010 S HAR BOR BLVD

ANAHEIMO CA 92805

$14774-0284$

HARNETT JAMES D

CHIEF DEPUTY SEALER

DEPT OF WEIGHTS E MEASURES

1010 S HAR BOR BLVD

ANAHEIM. CA 92805

$714774-0284$

LEFEUVRE RICHARD

DIST AGR ICULTURAL INSPECTOR DEPT OF WEIGHTS \& MEASURES

1010 S HAR BOR BLVD

ANAHEIM.CA 92805

$714774-0284$

MCNATT ROBERT F

INSPECTOR II

DEPT OF WE IGHTS 6 MEASURES

1010 S HAR BOR BLVD

ANAHEIM.CA 92805

$714774-0284$

MCROBERTS. TRAVIS C

DIST AGRICULTURAL INSPECTOR DEPT OF WE IGHTS E MEASURES

1010 S HARBOR BLVD

ANAHEIM. CA 92805

$714774-0284$

NELSON ROBERT E

INSPECTOR II

DEPT OF HEIGHTS $E$ MEASURES

1010 S HARBOR BLVD

ANAHEIM CA 92805

$714774-0284$

PAULUS. ARTHUR E

INSPECTOR II

DEPT OF WEIGHTS \& MEASURES

1010 S HARBOR BL VO

ANAHEIM. CA 92805

$714774-0284$

POOLE JAMES D

DEPT OF WE IGHTS \& MEASURES

1010 S HARBOR BLVD

ANAHEIM. CA 92805

$714774-0284$

SMITH. EDWARD H

INSPECTOR II

DEPT OF WEIGHTS $\&$ MEASURES

1010 S HAR BOR BLV VO

$714774-0284$

NCWM

STENTON. NEIL D

INSPECTOR II

DEPT OF WE IGHTS \& MEASURES

$1 O 10$ S HAR BDR BLVDD

ANAHEIM, CA 92805

$714774-0284$

IILLAE LILYE

DEPT OF WEIGHTS \& MEASURES

1010 S HARBOR BLVD

ANAHEIM. CA 92805

$714774-0284$

VILLALOBOS. ALVIE D

DEPUTY SEALER I

DEPT OF WE IGHTS \& MEASURES

1010 S HAR BDR BLVO

$714774-0284$ 
WYATT RCBERT E

DIST AGRICULTURAL INSPECTOR

DEPT DF WE GHTS \& MEASURES

1010 S HARBOR BLVD

ANAHEIM. CA 92805

$714774-0284$

\section{PLACER COUNTY}

HENDERSON JAMES C

INSPECTOR I I I

DEPT OF WEIGHTS \& MEASURES

11477 E AYENUE

AUBURN CA 95603

$916823-4371$

MITANI DOUGLAS $J$

DEPI OF WEIGHTS \& MEASURES

11477 E ÁVENUE

AUBURN CA 95603

$916823-4371$

PROCESSI RONALD W

INSPECTOR TI

DEPT OF WEIGHTS \& MEASURES

11477 E AVENUE

AU BURN: CA 95603

$916823-4371$

STARKO KENNETH \&

DEPT OF WEIGHTS \& MEASURES

11477 E AVENUE

AUB BURN CA 95603

$916823-4371$

WIL SON OPOHN H
SEALER OF WEIGHS \& MEASURES

PIACER COUNTY

IIST7 E AVE

AUBURN CA 95603

$916823-4371$

\section{PLUMAS E SIERRA COUNTY}

HORN MICHAEL R

MASPECTOR II

DEP OF WEIGHTS $\&$ MEASURES

ROUTE I BOX 230-A/FAIRGRNDS RD

QUINCY: CA 95971

916 283-1155

SURBER, FREDERICK H

SEARER OF HE IGHTS \& MEASURES

PLUAAS COUNTY

RT 1 BOX 230A

QUINCY, CA 95971

$916283-1155$

\section{RIVERSIDE COUNTY}

BENUENI STE CLEMENT

SEALER OF HEIGHTS \& MEASURES

4080 L MON ST/RM 19

RIVERSTDE CA 92501

CARBONE ERNEST J

DEPT OF WE TGHTS \& MEASURES

2950 WASHINGTON ST

RIVERSIDE CA 92504

NAHAS, ROGER $M$

INSPECTOR I

DEPT OF WEIGHTS $\$$ MEASURES

2950 WASHINGTON ST

RIVERSIDE, CA 92504

$716787-2620$

WELLS. ARTHUR $H$

INSPECTOR II

DEPT OF WEIGHTS \& MEASURES

2950 WASHINGTON ST

RIVERSIDE: CA

SACRAMENTO COUNTY

BEARDSLEY. NORMAN R

SENIOR INSPECTOR

4137 BRANCH CENTER RD

SACRAMENTO CA 95827

$916366-2003$

BROWN $\angle E L A N D$

DIRECTOR DF HEIGHTS \& MEASURES

SACRAMENTO COUNTY

4137 BRANCH CENTER RD

SACRAMENTO CA 95827

916 366-2003

DUNCAN D DONALD S

SR W E INSPECTOR

4137 BRANCH CENTER RD

$916366-2003$

FORBES JR, HILLIAM

DEPUTY DIRECTOR

4137 BRANCH CENTER RD

SACRAMENTO. CA 95827

$916366-2003$

GIBSON: CLARK

SR WEIGHTS E MEAS INSPECOTR

SACRAMENTO COUNTY

4137 BRANCH CENIER RD

SACRAMENTO. CA 95826

91.6 366-2003

GORDY CEECIL E

SACRAMENTO CA 95827

SACRAMENTO
$916366-2003$

KELSEY DORI

SR H \& INSPECTOR

4137 BRANCH CENTER RD

SACRAMENTO. CA 95827

$916366-2003$

SAN BENITO COUNTY

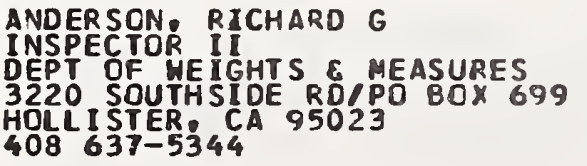

EDMONDSON. JOHN H

SEALER DF WEIGHTS AND MEASURES SAN BENITO COUNTY W AND M 3220 SOUTHSIDE RD HOLLISTER。CA 95023

$408637=5344$ 
MATULICH, PAUL J

INSPECYOR II

3220 SOUTHSIDE RD/PO BCX 699 HOLLI STER CA 95023

$408637-5344$

SPENCER JDSEPH H

DEPT OF WEIGHTS \& MEASURES 3220 SOUTHSIDE RD/PO BOX 699 HOLLISTER, CA 95023

$408637-5344$

TOGNAZZ INI MARK A SEELER CF WE IGHTS ${ }^{A}$ E MEASURES 3220 SOUTHSIDE RD/PO BOX 699 HOLLISTER, CA 95023

$408637-5344$

\section{IAN BERNAROINO COUNTY}

\section{CARLOS. HENRY $V$}

INSPECTOR II

DEPT OF HEIGHTS $\&$ MEASURES

777 E RIALTO AVE

SAN BERNARDINO, CA 92415

$714387-2105$

CHOJNACKI, CHARLES J

INSPECCTOR II

3200 ST

ONTARIO. CA 91762

$714988-1191$

CROYLE, BENNY D

INSPEC TOR II

777 E RALTO AVE M 92415

SAN BER NARDI

FARR, RCBERT W

COMPLIANCE DIVISION CHIEF

777 E RIALTO AVE

SAN BERNARDINO, CA 92415

$714387-2105$

HAHER STEVEN R

INEPT OF HEIGHTS \& MEASURES

777 E RIALTO AVE

SAN BERNARDINO. CA 92415

$714387-2105$

HANSON, GERALD H

DIRECTOR

SAN BERNARDINO COUNTY WTSIMEAS

777 ER IALTO AVE

SAN BER NARDINO, CA 92415

$714387-2136$

HARRIS WILLIAM E

INSPECTOR II

301 EAST MT VIEH

BARSTOH, CA 92311

$714256-9233$

LESTER, HARVEY A

INSPY OF WEIGHTS $\&$ MEASURES

77 E RIALTO AVE

SAN BER NARDINO, CA 92415

$714387-2105$
MARKUS JR, HENRY J

TS E MEAS INSPECTCR II

SAN BDNO CO WTS E MEASURES

SAN BERNARDINO. CA 92415

714 387-2105

MONTOYA, LARRY

INSPECTOR IIITS \& MEASURES

320 D ST

ONTARIOQ CA 91762

MORGAN TILFORD H

INSPECTOR II WHTS $E$ MEASURES

777 E RIALTO AVE

SAN BERNARDINO. CA 92415

$714387-2105$

PEARSALL R D

DEPT OF WE IGHTS 6 MEASURES

777 EIALTO AVE

SAN BERNARDINO, CA $\$ 2415$

$7-2105$

PERRY NEIL D

DEPT OF WEIGHTS $E$ MEASURES

777 E RIALTO AVE

SAN BERNARDINO. CA 92415

$714387-2105$

SAN DIEGO COUNTY

BENNETT:ED

DEPT OF WE IGHTS \& MEASURES

5555 OVERLAND AVE BLDG 3

SAN DIEGO CA 92123

714 565-3764

MBR

NCHM

KUSHMAN\& J EFFREY

INSPECTOR II

5555 OVERLAND AVE BLDG 3

SAN DIEGO: CA 92123

714 565-5764

THUNER, KATHLEEN A

AGRIC COMM SEALER HGTS $\&$ MSRS

MBR COUNTY OF SAN DIEGO 5555 OVERLAND AVENUE BLDG 3 SAN DIEGO. CA

619 565-5789

MBR

VINCENT: RICHARD W

INSP ECTOR II

DEPT OF WEIGHTS \& MEASURES

5555 OVERLAND AVE BLDG 3

SAN DIEGQ CA 92123

714 565-5764

SAN FRANCI SCO COUNTY

BROOKS, GR ANVI LLE L

INSPECTOR

DEPT OF WE IGHTS 8 MEASURES

501 ARMY ST SUITE 109 A

SAN FRANCISCO. CA 94124

415 285-5012

ENEA. HORACE $V$

INSPECTOR

DEPT OF WEIGHTS 8 MEASURES

501 ARMY ST SUITE $109-A$

SAN FRANCI SCO 
MINUCCIANI ELIO J

INSPECTON OF WEIGHT S EMEASURES COUNTY CF SAN FRANC ISCD

501 ARMY ST - 109-A

SAN FRANCI SCO. CA 94124

415 285-5012

WEETH GEORGE E

DEPT OF HEIGHTS $\varepsilon$ MEASURES

501 ARMY ST SUITE $109-A$

SAN FRANCI SCO

\section{SAN JCAQUIN COUNTY}

AGOSTINI, GUIDO

INSPECTOR DEIGHTS \& MEASURES

$P$ OOX 1809

STOCKTON CA 95201

ARGAIN, FRANK

SENIOR INSPECTOR

$P 080 \times 1809$

STOCKTON CCA 95201

EBY ERHIN B

MBR

1868 EAAEL TON AVE

209 $944-2225$

\section{FOILES RICHARD}

P C BOX 1809

STOCKTON CA 95201

$209982-4532$

HOLLISST JIM

DEPT OF WEIGHTS \& MEASURES

$8080 x 1809$

STCCKTON CA 95201

KIBBE, DCN

INSPECT CR

DEP WEIGHS \& MEASURES PO $80 X 1809$

STCCKTON CA 95201

LAVIERI: TONY

DEPT OF WEIGHTS $\&$ MEASURES

P O BOX 1809

STCCKTON CA 95201
$209982-4532$

PAR SONS FRED

INSPECTCR

DEPT DF WEIGHTS \& MEASURES

P O BOX 1809

STOCKTON CA 95201

RAY, LARRY G

DEPUTY SEALER

STOCKTON CA 95201
SAN LUIS OBISPO COUNTY

BOAZ BERNARR $M$

DEPUTY SEALER

DEPT OF WEIGHTS 6 MEASURES

2156 SIERRA WAY SUITE D

SAN LUIS. CA 93401

GREEK. RICHARD D

SEALER OF WEIGHTS $\&$ MEASURES SAN LUIS OBI ISPO COUNTY

2156 SIERRA HAY SU
SAN LUIS CA 93401
$805549-5910$

MILLSPAUGH LANCE C DEPT OF WE IGHTS \& MEASURES 2156 SIERRA WAY SUITE D SAN LUIS 8 CA 93401

MORRI SON STEPHEN F

CHIEF DEPUTY SEALER

DEPT OF WEIGHT \&

2156 SIERRA WAY SUITE

SAN LUIS CA 93401

NCWM

SAN MATEO COUNTY

GINILO. GEORGE

SEALER WEIGHTS \& MEASURES

REDWOOD CIIYY CA 94063

LOCKE. RAY

INSPECTOR III

DEPT DF WEIGHTS $\&$ MEASURES 728 HELLER ST AGR IC BLDG REDHOOD CITY. CA 94064

LOVE. THOMAS

DEPT OF WEIGHTS \& MEASURES 728 HELLER ST AGR IC BLDG

$415363-4700$. CA 94064

SLAITER, DONALD R

INSPECTOR III

DEPT OF WE IGHTS $E$ MEASURES

728 HELLER ST AGR IC BLDG

REDWOOD CITY, CA 94064

$415363-4700^{\circ}$

STECK, CHR IS

INSPECTOR III

DEPT OF WE IGHTS $\&$ MEASURES

728 HELLER ST AGR IC BLDG

REDWOOD CITY, CA 94064

SANTA BARBARA COUNTY

GIBSON. DENNIS HEIGHTS E MEASURES INSPECTOR 263 CAMINO DEL REMEDIO

SANTA BARBARA. CA 93110

805 964-1027

GILMAN RONALD M AGR I CULT TURAL COMMISSIONER

DEPT OF HEIGHTS E MEASURES

263 CAMINO DEL REMEDID

SANTA BARBARA. CA 93110

805 964-1027 
JOHNSON, GERALD L

INSPECYOR III

DEPT OF WEIGHTS $\&$ MEASURES

263 CAMINO DEL REMEDIO

805 964-1027

ROSSE RODERICK

INSPECTOR I

DEPT OF WEIGHTS $\&$ MEASURES

263 CAMINO DEL REMEDID

SANTA BARBARA, CA 93110 805 964-1027

WILEY: JAMES E

DEPT OF WEIGHTS E MEASURES 263 CAMINO DEL REMEDIO

SANTA BARBARA, CA 93110

805 964-1027

¿ANTA CLARA COUNTY

COQUPIA P DANIEL Q

DEPT OF WEIGHTS \& MEASURES

1553 BERGER DR BLDG 1

$408299-4701$

DEVAUGHNS LLOYD L

DEPT OF WE IGHTS \& MEASURES

1553 BERGER DR BLDG 1

SAN JOSE CA

ELLSWORTH, ELIZABETH A

DEPUTY SEALER

DEPT OF WEIGHTS \& MEASURES

1553 BERGER DR BLDG 1

SAN JOSE $299-4701$

GREENE, THOMAS W

INSPECICR II

DEPT OF WEIGHTS \& MEASURES

1553 BERGER DR BLDG 1

SAN JOSE: CA

LOHEE JCY L
INSPECTOR II
DEPT OF WEIGHTS E MEASURES
I5 53 BERGER DR BLOG I

SON JOSE CA 951 L2

PFEIFFER, MARIE E

DNSPECTCR III OF WE

DEPT OF WEIGHTS E MEASU

SAN JOSE CA 951 L2

408 299-4701

PRESTOSA PROSPERO

DEPT OF WEIGHTS 6 MEASURES

1553 BERGER DR BLDG 1

SAN JOSE CA 95112

408 299-4701

VACC ARRE JOHN

DEPT OF WEIGHTS \& MEASURES

1553 BERGER DR BLDG 1

SAN JOSE CA 95112

408 299-4701
VAN WASSENHDVE GREG

DIRECTOR OF WEIGHTS \& MEAS

SANTA CLARA COUNTY

1553 BERGER DR BLDG 1

SAN JOSE CA

SANTA CRUZ COUNTY

CRAFT: LEN ARD

DIRECTOR

WEIGHTS 8 MEASURES

1430 FREEDOM BLVD

WATSONVILLE: CA 95076

$408724-1149$

MOELLER, DAVIO

DEPUTY SEALER

DEPT OF WE IGHTS \& MEASURES

1430 FREEDOM BLVD

408 T24-II49 CA 95076

ROQF E PATRICK J

DEPT OF WEIGHTS \& MEASURES

1430 FREEDOM BLVD

WATSONVILLE CA 95076

SHASTA COUNTY

BROWN: RANDY A

AG E STANDARDS INVESTIGATOR I

DEPT OF WEIGHTS $\varepsilon$ MEASURES

3179 BECHELLI LANE SUITE 210

REDDING CA 96002

CASSADAY KIT L L

SHASTA COUNTY DEPT WTS $E$ MEAS

3179 BECHELLI LANE SUITE 210

REDDING CA 96002

$916225-5656$

GARR ISON KATHAR INE B

SHASTA COUNTY DEPT WTS E MEAS

3179 BECHELLI LANE SUITE 210

REDDING CA 96002

$916225-5656$

MCBROOME, DONALD E

AG ESTANDARDS INVE STIGATOR III SHASTA COUNTY DEPT WTS E MEAS 3179 BECHELLI LAN

$916225-5656$

MORTON JAMES G

AG ESTANOARDS INVESTIGATOR III

SHASTA COUNTY DEPT LTS E MEAS

3179 BECHELLI LANE SUITE 210

REDDING CA 96002

$916225-5656$

PRICE RICHARO B

AG ESTANDAROS INYESTIGATOR III

SHASTA COUNTY DEPT WTS E MEAS

REDDING CA 96002

$916225-5656$

WRI GHT - KENNETH W

SEALER OF WEIGHTS \& MEASURES

SHASTA CO DEPT DF AGRICULTURE

3179 BECHELLI LANE STE 210

REDDING $91625-565696002$ 


\section{SISKIYCU COUNTY}

CORBIN. KENNETH L

INSPECTOR I IOTSS \& MEASURES

$525 S$ FCOTHILL OR

YREKA CA 96097

HALE EOMOND W WTS E MEASURES

SISKIYOU COUNTY DEPJ OF AGRI 525 SO FOOTHILL DRIVE

YREKA. CA 96097

$916842-3531 \times 280$

JANSSENE DONALO L

DEPT OF WEIGHTS \& MEASURES

525 S FOCOTHILL DR

$916842-3531 \times 280$

KRUEGER WILLIAM A

DEPT OF WEIGHTS \& MEASURES

525 S FOCTHILL DR

YREKA CA 96097
$916842-3531 \times 280$

MASSEY JR, JAMES R

SENIOR DEPUTY SEALER

DEPT OF WEIGHTS $\&$ MEASURES

525 S FOCOTHIL DR

$916842-3531 \times 280$

SOLANC COUNTY

BAKER SIDNEY E

DEPT OF WEIGHTS \& MEASURES

560 FALRGROUNDS DR

VALLEJO 70755 CA

KELLER R ROGER

OEPT OF HEIGHTS $\&$ MEASURES

560 FAI RGROUNDS DR

$707553-5281$

KENNELLALBERT J

INSPEC TOR III

DEPT DF WEIGHTS \& MEASURES

560 FAIRGROUNDS DR

VALLEJ $553-5281$

MUSGROVER JULIUS E

INSPECTCR II

560 FAIRGROUNDS DR

\begin{tabular}{l} 
VALLE \\
707 \\
\hline $53-5281$
\end{tabular}

OL SON WILLIAMC

SEALER CF WEIGHTS E MEA SURES WE IGHT S MEEASURES SOLANO COUNTY 560 FAIRGROUNDS ORIVE

VALLEJO

\section{SONOMA COUNTY}

\section{BURGESS CRAIG}

SONCMA COUNTY WTS $\&$ MEASURES

2680 VENTURA AVE ROLOM 100

SANTA ROSA $707527-2548$ CA 95401
MBR

NCWM

CAPORALE: CLIFFORO R

INSPECTDR II

DEPT OF HEIGHTS \& MEASURES

2688 VENTURA AVE

SANTA ROSA. CA 95401

$707527-2548$

FRENK, LEE $V$

INSPECTOR II

2688 VENTURA AVE

SANTA ROSA CA 95401

$707527-2548$

HANSON, MARK H

INSPECTOR III

SONOMA COUNTY WTS $E$ MEASURES

2688 VENTURA AVE RCOM 100

SANTA ROSA CA 95401

SANDRETTO, RAYMOND $G$

CHIEF DEPUTY SEALER

DEPT OF WEIGHT S \& MEASURES

2688 VENTURA AVE RM $100 S$

SANTA ROSA. CA 95401

$707527-2548$

URBAN. EDWARD

SONOMA COUNTY

2604 VENTURA AVE RM 101

SANTA ROSA. CA 95401

$707527-2371$

\section{STANISLAUS COUNTY}

KELLEY• ROBERT

SENIOR INSPECTOR

DEPT OF WEIGHTS \& MEASURES

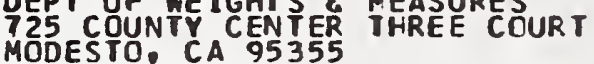

$209571-6587$

KLINK. JOHN N

DEP SEALER OF HTS \& MEAS

725 CO CENTER \#3 CT

$209571-6587$

MAHAN. KEITH

COUNTY SEALER

DEPT OF WEIGHTS \& MEASURES

725 COUNTY CENTER THREE COURT MOOE STO CA 2095

NEL SON

SR WEIGHTS \& MEAS INSPECTCR 725 COUNTY CENTER THREE COURT MODESTO. CA 95355

$209571-6587$

PEZZONI L L ANETIE

SR WEIGHIS \& MEAS INSPECTOR 725 COUNTY CENTER

$209571-6587$

SUTTER COUNTY

AHLERS RALLIN C

ASSISTANT DIRECTOR

DEPT OF WE IGHTS $\&$ MEASURES

142 GARDEN HIGHWAY

$916741-7500$

MILLER GERALD E

INSPECTOR II

DEPT OF WEIGHTS $\&$ NEASURES

142 GAROEN HIGHWAY

YUBA CITY. CA 95991

916 741-7500 
PERRIN JR, ALFRED C

DIRECTOR

SUTTER COUNTY WEIGHTS \& MEAS

142 GARDEN HIGHWAY

YUBA CI TY CA 95991

TEHAMA COUNTY

BQVEE GARY C INSPECTOR II

WEIGHTS E MEAS

RED BLUFF CA 96080
$916527-4504$

HILLODCNALO R

TEHAMA COUNTY

PO BOX 381760 HALNUT ST

RED BLUFF, CA 96080

$916527-4504$

WILLIAMS B BILLLL S INSPECTOR II P $80 X^{3} 38$

RED BLUFF, CA 96080

\section{TRINITY COUNTY}

SEALER

TRINITY CO HEIGHTS \& MEASURES P C BOX 1466

WEAVERVILLE: CA 96093

$916623-1356$

TULARE COUNTY

KUNKEL GARY $N$

INSPECTER II

DEPT OF WEIGHTS $\&$ MEASURES

COUNTY CIVIC CENTER-AGRIC BLDG YISALIA. CA 93291

$209733-6391$

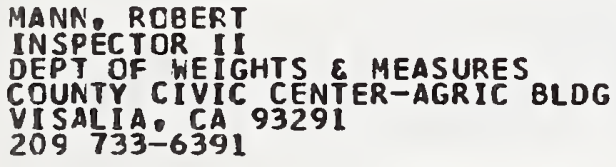

PENNINGTON, JCHN O

SEALER TULARE COUNTY

AGR ICUL TURAL BLDG

COUNTY CIVIL CENTER

VISALIA. CA

MBR

MBR

VILLICANA. MANUEL

INSPECTCR II

DEPT OF WEIGHTS $\varepsilon$ MEASURES

CDUNTY CIVIC CENTER-AGRIC BLDG

VISALIA, CA 93291

$209733-6391$

WALLACE, RICHARD B

INSPECTOR III

DEPT OF WEIGHTS \& MEASURES

COUNTY CIVIC CENTER-AGRIC BLDG VISALIA: CA 93291

$209733-6391$

TUOL UMNE COUNTY

ANZAR, MARIAN

INSPECTOR III

DEPT OF WEIGHTS \& MEASURES

2 SCUTH GREEN ST

$209533-5691$
BENINCASA. GERALD

DIRECTOR OF WEIGHTS \& MEASURES DEPARMEN OF WEIGHTS $\&$ MEAS SONORTH GREEN ST

$209533-5691$

\section{VENTURA COUNTY}

BRISTER - JAMES A

WEIGHTS $\&$ MEASURES TECHNICIAN DEPT OF WEIGHTS E MEASURES

800 S VICTORIA AVE

VENTURA. CA 93009

$805654-2444$

COBOS JR MARTIN E DEPT OF WEIGHTS \& MEASURES

800 S VICTORIA AVE

VENTURA. CA 93009

$805654-2444$

JORDAN PAUL D

INSPECTOR

DEPT OF WEIGHTS $E$ MEASURES

800 S VICTOR IA AVEE

VENTURA. CA 93009

805 654-2444

KORTH. HILLIAM H

DIRECTOR

VENTURA CNTY WEIGHTS \& MEASURE 800 S VICTORIA AVE

VENTURA. CA 93009

$805654-2446$

LAUBACHER, DAVID F

SUPERVIS ING INSPECTCR

OEPT OF WEIGHTS E MEASURES

800 SOUTH VICTORIA

VENTURA. CA 93009

$805654-2444$

LUCKING III HILLIAM A

WEIGHTS $\varepsilon$ MEASURES TECHNICIAN DEPT OF WEIGHTS \& MEASURES BOO S VICTORIA AVE

VENTURA. CA 93009

$805 \quad 654-2444$

WALKER NAVEEN B

INSPECTOR

COUNTY OF YENTURA

BOO S VICTORIA

VENTURA. CA

YOLO COUNTY

CARL FRANK F E

DEPT OF HEIGHTS E MEASURES

HOODLAND: CA 95695

916 666-8140

DOANE CARL

DEPT OF WEIGHTS \& MEASURES

70 COTTONHOOD ST

HOODLAND. CA 95695

$916666-8140$

OULLUM, MARSHALL A

INSPECTOR III

DEPT OF WE IGHTS $E$ MEASURES

70 COTTONWOOD ST

HDODLAND. CA 95695

916 666-8140
MBR

NCWH 
PERKINS, RAYMOND

YIRECTOR OF WEIGHTS \& MEASURES

70 COTTCNWODD ST

HDOLLAND CA 95695

WALLACE, DAVIO

CHIEFMEASURMNT STANDAROS SECT 3125 WYANDOT

$916666-8140$

DENVER, CD 80220

YUBA COUNTY

ENGLE, BERNIE

$93814 T \mathrm{H}$ ST

MARYSVILLE: CA 95901

COLORADO

STATE

BROOKS, DONALD

MEA SUREMENT STANDAROS SECTION

3125 WYANDOT

303 DER:CO 80211

BRZOTICKY, FRANK

CHIEF METROLGIST

DEPARTMENT OF AGRICULTURE

3125 HYANDOT

DENVER, CO 80211

CASH HILLIAM F

MEASUREMENT SSTANOARDS SECTION

COLCRADO DEPT OF AGRICULTURE

3125 HYANDOT

DENVER, CO 80211
$303866-2845$

HOGSETT CLYDE

MEASUREMENT STANDARDS SECTION COLCRADC DEPT OF AGR ICULTURE 3125 WYANDOT

DENVER CO 80211
$303866-2845$

PAULSON ALAN

MEA SURE HENT STANDARDS SECTION

COLORADO DEPT OF AGRICULTURE

3125 WYANDOT

DENVER, CO 80211
$303866-2845$

POWEL PIKE
STATE INSPECTOR OF OILS
COLCRAOOSTATE OIL INSP SECT

$M B R$

DENVER COND 80216

NCWN

$303289-5643$

ROMERO, BILL

MEA SUREMENT STANDARDS SECTION

COLCRADO DEPT OF AGRICULTURE

3125 WVANDOT

DENVER CO 80211
$303866-2845$

SCOFIELD $L E E$

MEASUREMENT STANDARDS SECTION

3125 WYANDOT

DEN VER, CO 80211

WAGNER, JERRY

METROLÓGIST

MEA SUREMENT STANDARDS SECTION

(1)

DENVER CO 80211
$303866-2845$

\section{CONNECTICUT}

STATE

CRISTELLI. DAVID

INSPECTOR

HEIGHTS \& MEASURES OIVISION

STATE OFFICE BLDG/CONS PROT

HARTFORD
$203566-4778$

DONAHOE, WILLIAM

WEIGHTS \& MEASURES OIV

STATE OFFICE BLDG

203 566-4778

ORAKE: ELLIOTT

INSPECTOR

HEIGHTS \& MEASURES OIVISION

STATE OFFICE BLDG/CONS PROT

HARTFORD: CT 06106

$203566-4778$

DYNIA. MICHAEL

METROLOGIST

WEIGHTS ANO MEASURES

RM G- I7 ST OFF BL 165 CAPITOL HARTFORO C CT 06106

FORREST: FRANK

INSPECTOR

WEIGHTS $\&$ MEASURES DIVISION

STATE OFFICE BLOG/CDNS PROT

HARTFORD: CT 06106

FOR TIN, JOSEPH

INSPECTOR

WEIGHTS $E$ MEASURES DIVISION

STAT DFFICE BLDG / CONS PRDT

$203566-4778$

HAYES: JOHN

WEIGHTS E MEASURES OIVISION

STATE OFFICE BLDG

HARTFORD. CT 06106

$203566-4778$

HESLIN. MARY M

COMMISSIONER

CT DEPT CONSUMER PRCTECTION ST OFFICE BLDG 165 CAPITOL AVE 203 566-4999

HUTCHINSCN. RONALD

SENIOR INSPECTOR

WEIGHTS E MEASURES DIVISION

S A A T

$203 \quad 566-4778$ 
KALENTKOWSKI RAYMOND SENIOR INSPECTOR
HEIGHTS E MEASURES DIVISION
STATE DFFICE BLDG/CONS PROT HARTFORD. CT 06106

203 566-4778

KOZIY. DAVIO

INSPECTOR

WEIGHTS \& MEASURES DIVISION

STATE OFFICE BLDG

$203566-4778$

NEL SON ALLAN M

CHIEF WEIGHTS \& MEASURES

165 CAPITOL AVE ROOM GI7

HARTFORD CT 06106

$203566-5230$

\section{PAKER JAY \\ WEIGHTS \& MEASURES DIVISION \\ STATE OFFICE BLDG \\ HARTFORD. CT 06106 \\ $203566-4778$ \\ PHELPS, THOMAS \\ INSPECTCR \\ WEIGHTS \& MEASURES DIVISION \\ STATE OFFICE BLDG \\ HARIFORD CT 06106 \\ $203566-4778$}

SLAMON JR. WILLIAM J M DIVISION ASSISTANT CHIEF WE M DIVIS STATE OFF BLDG/165 CAPITOL AVE HARTFORD CT 06106

$203566-5230$

STEPHENS: MARK

WEIGHTS $\&$ MEASURES DIVISION

STATE OFFICE BLDG

$203566-4778$

THOMPSON, JOHN

INSPECTOR

WEIGHTS E MEASURES DIVISION

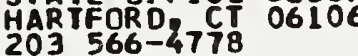

WILSON PETER

WE IGHTS \& MEASURES DIVISION

STATE OFFICE BLDG/CDNS PROT HARTFORD: CT 06106

$203566-4778$

CITY: ANSONIA

SOKOLNICKI, ALBIN H

CITY SEALER

WEIGHTS $E$ MEASURES DIVISIDN

CITY HALL

ANSONIA: CT 06401

\section{CITY: BRIDGEPORT}

DIMENNA JOHN D

DEPUTY SEALER OF WTS \& MEAS

CITY OF BRIDGEPORT

45 LYONS ST

BRIDGEPORT CT 06604
INNACELLO, ANTHONY

WEIGHTS $\&$ MEASURES DIVISION

Ciry

$203576-7596$

PAOLETTI DANIEL $R$

DEPUTY SEALER

HEIGHTS

203 BR DGORT 3 CT 06610

MBR

CITY: BRISTOL

LEONE SR JOHN J

WEIGHTS E MEASURES DIVISION

CITY HALL

BRISTOL:CT 06010

CITY: DERBY

ORAZIETTI FLAVIO

CIIY SEALER

WEIGHTS \& MEASURES DIVISION

CITY HALL

DERBY ${ }_{3}$ CT 086418

CITY: EAST HARTFORD

MBR

BONADIES, DOMINIC

TOWN SEALER

76 CIPPOLA OR

EAST HARTFORD, CT 06118

203 569-0463

CITY: GREENWICH

BELMONT AOFTHONY F

SEALER OF WEIGHTS

IOI FIELD POINT RD

GREENWICH.CT 06830

$203622-7800$

CITY: HARTFORD

MOKRYCKI JOHN

CITY SEALEP

WEIGHTS E MEASURES DIVISION

CITY HALL

HARTFORD: CI 06103

$203722-6370$

CITY: MIDOLETOWN

TOMMASI GUY J

SEALER OF HEIGHTS 6 MEASURES

CITY DF MI DDLETOWN

MTDDLETOWN 8 CT 06457

CITY: NEW BRITAIN

PEARSON, BRYANT

SEALER OF WEIGHTS \& MEASURES

CITY OF NEH BRITAIN CONN

CITY HALL 27 W MAIN ST

NEW BRITAIN CT 06051

$203224-2491 \times 230$
MBR

NCWM

MBR

NCWM

MBR

NCWM 
CITY: NEh HAVEN

CIOCIOLA. CHRISTINE

SEALER CF WEIGHTS E MEASURES

CITY OF NEW HAVEN

I STATE ST

N 203 HAVEN $787-836906510$

PRAKLIS PHILIP

WEIGHYS \& MEASURES DIVISION

NEW HAYEN CT 06510

STEPHENS, WILBUR

INSPECTOR

WE IGHTS \& MEASURES DIVISION

NEW HAVEN CT 06510

CITY: NORWALK

ANASTAS IA PETE S

SEALER OF WEIGHTS $E$ MEASURES

DEE ST AVE

NORWALK. CT 06850

203 854-7791 $\times 291$

CITY: SHELTON

CAREY, SARA

TOWN SE ALER

WE IGHTS $\varepsilon$ MEASURES DIVISION

903 HOWE ST

SHELTON, CT 06484

CITY: STAMFORD

KOZIOL ALFONS F

SEALER" OF WEIGHTS AND MEASURES

429 ATLANTIC STCITY HALL

$203358-4140 \times 695$

CITY: TORRINGTON

AESCHLEMANN, KEITH

CITY SEALER

WEIGHTS $\&$ MEASURES DIVISION

CITY HALL

TORRING TCN CT 06790

CITY: WALLINGFORD

BRYAND\& IILFRED

TOWN SEALER

WEIGHTS $E$ MEASURES DIVISION

32 NICHOL AS RD

WALLINGFORD, CT 06492

CITY: WATERBURY

TRAYER JR HAROLD J

WE GHTS E MEASURES DIVISION CITY HALL

WATERBURY, CT 06702

MBR

NCWM

MBR
CITY: WEST HAVEN

NCWH

TAMBORINI。EDWARD

SEALER

WEIGHTS 6 MEASURES DIVISION

HEST HAVEN. CT 06516

OELAHARE

STATE

BAIRD. DAYIOL

METROLOGIST

WEIGHTS 6 MEASURES SECTION 2320 S DUPONT HWY

$302736-4811$

CLEAYER, CHRIS

WEIGHTS 6 MEASURES INSPECTOR STATE OF DELAWARE

DOVER DE 19901

$302736-4811$

CORDREY. MARK

WEIGHTS $\&$ MEASURES INSPECTOR 2320 S DUPONT HWY

2320 S DUPONT H

$302736-4811$

FREOERICK, CHARLES

WEIGHTS \& MEASURES INSPECTOR STATE OF DELAWARE

$302736-4811$

KEELEY EUGENE

SUPERVISC

DEL AWARE HEIGHTS ANO MEASURES 2320 SOUTH DUPONT HIGHWAY

DOVER, OE 19903

$302736-4811 \times 37$

LAGEMANN . WILLIAM

WEIGHTS $\&$ MEASURES INSPECTOR STATE OF DELAWARE $2320 S$ DUPONT HWY DOVER. DE 19901

$302736-4811$

CITY: DISTRICT OF COLUMBIA

BOND. MARGARET B

INSPECTOR HEIGHTS $\&$ MEASURES

DISTRICT OF COLUMB IA

WASHINGTON D DC 20020

202 767-7923

DOUGLAS. HAROLDJ

INSPECTOR WEIGHTS $\&$ MEASURES

DISTRICT OF COLUMBIA

110 U ST SE

WASHINGTON. OC 20020

MATTHEHS, WILLIAM A

INSPECTOR WEIGHTS E MEASURES OISTRICT OF COLUMB IA

1110 ST SE

WASHINGTON 202 DC 20020

$203574-6856$ 
MAXXELLE EARL EEAS $E$ MARKET QR DEFT OF CONSUMER \& REGL AFFRS IIIO ST SE

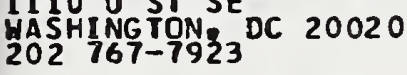

MURRAY JR. ERANCIS J MURRAY JR, FRANCIS J J
INSPECTCR WEIGHTS E MEASURES
DISIRICT OF COLUMB A $1110 \mathrm{U} S T$ S HASHINGTON DC 20020

\section{STATE}

ADAMS, GEORGE

CALIBRATION

FLA DEPT OF AGRIC E C S S STA FT LAUDERDALE

\section{AOAMS, WILLIAM}

FLA DEPT OF AGRIC \& C S HAOC SHERIDAN ST \#25

\section{ALDAY $B$ TALTER \\ FL OEPT OF AGRIC \& C S TALLASSEE. FL 32303}

\section{ALLEN KIM S \\ WEIGHTS G MEASURES C C S \\ 3125 CONNER BLVD \\ TALLAHASSEE FL 32399 \\ 904 488-9140}

\section{ALLREDGE, WILUIS \\ PETRQLEUM INSPECTOR \\ FLA DEPT OF AGRIC \& C S 3925 AY ENUE T NW}

WINTER HAVEN. FL 33881

ALPHIN, R M

CALIBRATION

FLA DEPT OF AGRIC \& C S

RT 9 BOX 220

TALLAHASSEE. FL 32303

BALLE WAYNE FLA DEPT OF AGRIC \& C S

3125 CONNER BLVD

TALLAHASSEE, FL 32399

$904488-9140$

BALLARD, BOB

$\angle P$ METERS/PETROLEUM

ROUTE I 6 BOX 30

TALLAHASSEEः FL 32304

BARNES, CHARLES E

FLA DEPT OF AGRIC \& C S

3125 CONNER BLVD

TALLAHASSEE FL 32399

$904488-9140$

BARNES, JOHN

CALIBRATION

FLA DEPT OF AGRIC \& C S

2821 HA GON WHEEL CIRCLE

ORLANDO. FL 32822
BLANCHARD, ROBERT

PETROLEUM INSPECTOR

FLA DEPT DF AGRIC \& C S

PO BOX 3602

PANAMA CITY. FL 32401

BOWEN. BEN

LAB CHIEF BUR DF PETRO INSP

FLA DEPT OF AGRIC E C S

3125 CONNER BLYD BLDG

BREEDING STAN

WEIGHTS \& MEASURES

FLA DEPT OF AGRIC \& C S

3125 CONNER BLVD

TALLAHASSEE. FL 32301

BRISTOL, JOHN

PETROLEUM INSPECTOR

FLA DEPT OF AGRIC \& C S

7080 SW $16 \mathrm{rH} \mathrm{ST}$

PLANTATION. FL 33317

BRUMBLES. ROBERTA

WEIGHTS \& MEASURES \& C S

3125 CONNER BLVD

TALLAHASSEE FL 32399

$904488-9140$

BYRD. RICHARD E

WEIGHTS \& MEASURES

FLA DEPT DF AGRIC E C S

3125 CONNER BLVD

TALLAHASSEE FL 32399

CAGLE.JAMES

PETROLEUM INSPECTOR

FLA DEPT OF AGRIC E C S

8 GARDEN ST APT $107-R$

TEQUESTA. FL 33458

CARNEY. PHILLIP

PETROLEUM INSPECTOR

FLA DEPT OF AGRIC E C S

551 NW $48 T H$ AVE

DELRAY BEACH. FL 33445

904 488-9140

CATE, W W

PETROLEUM INSPECTOR

FLA DEPT OF AGRIC $\&$ C S $425 \mathrm{~N}$ LOCH VIEW TERR

CRYSTAL RIVER, FL 32629

$904488-9140$

CHAIRES THOMAS S

SUPERVISOR

DIV OF STANDARDS

PO BOX 1609

BRADENTON: FL 33506

$813792-4670$

COOK. MICHAEL D

HEIGHTS E MEASURES \& C S 3125 CONNER BLVD

TALLAHASSEE. FL 32399

$904488-9140$

COOLEY. JOSEPH M

HEIGHTS \& MEASURES

FLA DEPT OF AGRIC E C S 3125 CONNER BLVD

TALLAHASSEE FL 32301

CRAFA. HENRY $J$

PETROLEUM INSPECTOR

FLA OEPT OF AGRIC E C S 6627 4TH AVEN

ST PETER SBURG

FL 33710 
CRUCET, ANTHONY

WEIGHTS \& MEASURES

FLA DEPT OF AGRIC E C S

3125 CONNER BLYD 32301

D ARCY CARLOS U⿱口卄:S

FLA DEPT OF AGRIC \& C S

3125 CONNER BLVD

TALLAHA SSEE. FL 32301

DARROW. BOB

SUPERVI SOR

DEPT OF AGR DIV OF STDS

TALLAHASSEE FL $32399^{\circ}$

$904488-9295$

DAYIS JAMES E

WEIGHTS \& MEASURES SPECIALIST

2015 SE 9TH TERR

CAPE CORAL FL 33904

DAVIS, THEODORE

WEIGHYS $E$ MEASURES

FLA DEPT OF AGRIC \& C S

3125 CONNER BLVD

TALLAHASSEE: FL 32399

$904488-9140$

DERCCHER, BRUCE

PETROLEUA INSPECTOR C S

937 DOGMOOD ROAD

HEST PALM: FL 33406

OUPREE $J R$ BILLY

AGR ICUL TURE PRDDUCTS INSPECTOR

MBR

FLA DEPT AGR DIV HEIGHTSE MEAS

NCWM

$P$ O BOX O5T548
WEST PALM FL 33405
$305659-5582$

EASTER MICHAEL

PETROLE UA INSPECTOR

FLA DEPT OF AGRIC \& C S

7 EAST VIRGIL

APOPKA, FL 32712

\section{EDELEN, BARRIE $M$}

WEIGHTS \& MEASURES $C$ DEPT OF AGRIC \&

3125 CONNER BLVD

TALLAHASSEE. FL 32399

EDWARDS. STEVE

PETROLEUM INSPECTOR

FLA DEPJ OF AGRIC E C S

JACKSONVILLE. FL 32205

\section{FICRELLI. PAT}

PETROLEUM INSPECTOR

FLA DEPT OF AGRIC E C S

18380 NE 2OTH PLAC

$904488-9140$

FOROROL WHU HNSPECTOR

FLA DEPT OF AGRIC \& C S JACKSONVILLE. FL 32244

JACKSONVILLE.
$906488-9140$
FREDERICK, FINN

PETROLEUM INSPECTOR

FLA DEPT OF AGRIC \& C S

PO BOX 1734

SEBRING FL 33871

GARREIT \& T

PETROLEUM INSPECTOR

FLA DEPT OF AGRIC E C S

125 NORTH ENZOR RD

CRESTVIEW. FL 32526

GARRIS, ROBERT E

WEIGHTS $\&$ MEASURES $C$

3125 CONNER BLVD

TALLAHASSEE FL 32399

$904488-9140$

GENTRY: PAUL

HEIGHTS \& MEASURES \& C S

3125 CONNER BLVD

TALLAHASSEE。FL 32301

GRAU RQR ROBERT LI OF STDS

DEPT OF AGRIC

3125 CONNER BLVD ILAB CCMPLEX

TALLAHASSEE: FL 32399

GRAY MAXWELL H

FLA DEPT OF AGRIC

TALLAHASSEE, FL 32399
$904488-9140$

NI

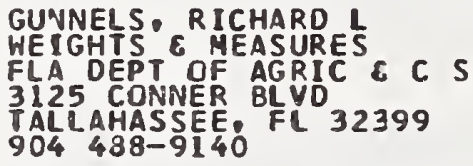

HADDER。 STEVE

PETROLEUM INSPECTOR

FLA DEPT OF AGRIC \& C S 1021 REGENCY DR

HAIR JR • HERBERT

WEIGHTS \& MEASURES

FLA DEPT OF AGRIC \& C S

3125 CONNER BL VD

TALLAHASSEE⿱
$90448 B-9140$

HALLLD JAMES SNSECTOR

FLA OEPT OF AGRIC E C S

803 ESSEX DR

TALLAHASSEE. FL 32304

HARDEE CURTISS E

FLA DEPTOF AGRIC E C S TAMPA: $F L, 33615$

$813884-8132$

HEIECK, KARL H

WEIGHTS \& MEASURES \& C S

3125 CONNER BLVD

TALLAHASSEE FL 32399

$904488-9140$

HERRING RANDY

PETROLEUM INSPECTOR

FLA DEPT OF AGRIC E C S

ROUTE 4 BOX 368

ALACHUA, FL 32615 
HEXTELL • PAUL

EIA DEPT OF AGRIC \& C S

3125 CONNER BLVD

PALLAHASSEE. FL 32301

HOWARD, BILLY R

WEIGHTS \& MEA SURES

FLA DEPT OF AGRIC \& C S

3125 CONNER BLVD

TALLAHASSEE FL 32399

$904488-9140$

HUNTER, EOWARO L

WEIGHTS E MEASURES

FLA DEPT OF AGRIC \& C S

3125 CONNER BLVD

TALLAHASSEE. FL 32301

INGRAHAM, ROOSEVELT

PETROLEUM INSPECTOR

FLA DEPT OF AGRIC \& C S

740 NH 38TH AVE

FT LAUDERDALE. FL 33311

JACKSON JR, HARDIE D

WEIGHTS $\&$ MEASURES

FLA DEPT OF AGRIC \& C S

TALLAHASSEE FL 32399

904 488-9140

MBR

JEFFRIES JACK Y

FLA DEPT OF AGRIC \& C S

NCWM

7910 ALAFIA DR

RIVERVIEW. FL 33569

JOHNSON D RICHARD K

WEICHTS 6 MEASURES $\&$ C

3125 CONNER BLVD

TALLAHASSEE. FL 32399

$904488-9140$

JONES, ROBERT S HEI GHY S E MEA SURES
FLA DEPT OF AGRIC \& C S
3125 CONNER BLVD

TALLAHASSEE. FL 32301

JONES WILLIAM

WEIGHYS \& MEASURES

FLA DEPT OF AGRIC \& C S

3125 CONNER BLVD

904 488-9140 FL 32399

KERRIGAN BERNARD J

FEIGHTS DEP MFASURES $C S$

3125 CONNER BLVD

TALLAHASSEE FL 32399

$904488-9140$

KOEPPEN EDWARD T

SENIOR METROLOGIST

3125 CONNER BLDG
TALLAHASSEE FL 32399
$904488-9395$

LAWSCN, ROBERTA

PETROLEUM INSPECTOR

FLA DEPT OF AGRIC \& C S

4634 FIRESTONE RD

JACKSONVILLE, FL 32210
LENNAN WILLIAH

PETROLEUA INSPECTOR 740 HUNAN OF AGRIC E C S

PALM BAY. FL 32907

LEVERETIE ROBER

PETROLEUM INSPECTOR

FLA DEPT OF AGRIC \& C S 8624 WOQDR IDGE RO
TAMPA. FL 33619

LIRAKIS. NICK

PETROLEUM INSPECTOR 13701 SW 487 T ST

LITTLE. MICKEY

PETRDLEUH INSPECTOR

5425 SAN JOSE DR

SARASOTA. FL 33580

LOPEZ. HENRY L

WEIGHTS \& MEASURES

FLA DEPT OF AGRIC \& C S

3125 CONNER BLVD

TALLAHAS SEE FL 32399

904 488-9140

MACIVOR, KENNETH

PETROLEUM INSPECTOR

FLA DEPT OF AGRIC E C S 5950 SW 172 ND AVE

33331

MCDONALD, PATRICIA

FLA DEPT OF AGRIC \& C S

3125 CONNER BLVD

TALLAHASSE E. FL 32399

$904488-9140$

MCKENZIE LINDAH

HEIGHTS ${ }^{\circ}$ MEASURES \& C S 3125 CONNER BLVD

TALLAHASSEE FL 32399
904 488-9140

MUELLE. MI GUEL

WEIGHTS 6 MEASURES

FLA DEPT OF AGRIC \& C S 3125 CONNER BLVD

TALLAHASSEE, FL 32301

MUNOZ. BILL

PETROLEUM INSPECTOR C S 230 EAST $58 T H$ ST

MYR ICK, RALPH

PETRDLEUM INSPECTOR C 829 CAREY DR

SOUTH DAYTONA, FL 32019

NIXON. HAYWOOD

SAMPLE TAKER/PETROLEUM FLA DEPT OF AGRIC \& C S ROUTE 2 BOX 58BDA

CRESTVIEW.FL 32536

OLIVE, CHARLES

PETROLEUM INSPECTOR

FLA DEPT OF AGRIC \& C S

LAKE CITY, FL 32055 
PALMER, FERNANDO

WE IGHTS $\&$ MEASURES

FLA DEPT OF AGRIC \& C S 3125 CONNER BLVO

TALLAHASSEE, FL 32301

PARDO ANDRES

HEIGH GHS \& MEASURES C S 3125 CONNER BLVD

TALLAHASSEE, FL 32399

$904488-9140$

PEACOCK • JERRY E

WEIGHTS G MEASURES
FLA DEPT OF AGRIC \& C S
3125 CONNER BLVD

TALLAHASSEE, FL 32399

$904488-9140$

POPCVICS. FRANK

WEI GHTS 6 MEASURES

FLA DEPT OF AGRIC \& C S

3125 CONNER BLVD

TALLAHASSEE FL 32399

POULOS TOM

FLA DEPT OF AGRIC \& C S 1521 SW $127 H$ AVE

REEC JR J JOHN

WEIGHTS $\&$ MEASURES

FLA DEPT OF AGRIC \& C S

3125 CONNER BLVD

TALLAHASSEE: FL 32399

REYNOLOS LOUIS

PETROLEUM INSPECTOR

FLA DEPT OF AGRIC \& C S 5310 SW 162ND AVE

ROLLE, CARROLL

CALIBRATION

9300 NW 12TH AVE

MIAMI? FL 33150

ROLLE STEVE

WEIGHTS \& MEASURES

FLA DEPT OF AGRIC \& C S

TALLAHASSEE. FL 32301

ROSEN, GLENN

PETROLEUM INSPECTOR

FLA DEP T OF AGRIC \& C S

PO BOX 680

CHRISTMAS. FL 32709

SCHCNS, CLIFFCRD

PETROLEUM INSPECTOR C S

CAPE CORAL. FL 33904

SCHROEDER , OARCY

WEIGHTS $E$ MEASURES

FLA OEPT OF AGRIC \& C S

TALLAHASSEE, FL 32301

SEHARD, TOM

CALIBRATION

FLA DEPT OF AGRIC \& C S

ORLANDO. FL 32809
SHIPMAN, RALPH F

SCALE MAINTENANCE TECH II FLORIDA DEPT OF TRANSPORTATION PO BOX 2877

LAKE CITY FL 32056

SHR INER, HARRY

CAL IBRAT ION

FLA DEPT OF AGRIC $\&$ C S

1211 DAGWOOD HAY

RIVERVIEH. FL 33565

SKALKOWSKI - STANLEY

DEPUTY DIRECTOR

CONSUMER PROTECTION DIVISION

$44 W$ FLAGLER ST SUITE 2303

MIAMI 305 FL 33130

SMI TH BARRY E

WEI GHTS $C$ MEASURES

FLA DEPT OF AGRIC $\&$ C $S$

3125 CONNER BLVD

TALLAHASSEE. FL 32301

THOMPSON FREDDIE

PETROLEUM INSPECTOR

FLA DEPT OF AGRIC E C S

BO8 AMBERJACK LANE

32233

TILLER III WILLIAM C

SUPER VISOR FEPT DF ASR OIV STD

3125 CONNER BLVD

TALLAHASSEE. FL 32399

904 488-9140

TURNER $J$ JOE

PETROLEUM INSPECTOR

FLA DEPT OF AGRIC E C S

TAMPA. FL 33615

YALDES FRANK

PETROLEUM INSPECTOR

FLA DEPT OF AGRIC \& C S

PO BDX 541752

OPA-LOCKA. FL 33054

VANNESS $M M$

PETROLEUM INSPECTOR

FLA DEPT OF AGRIC E C S

PCALA. FL 32678

HAGNER DOUGLAS

CALIBRATION AGRIC\&CS

ROUTE 9 BOX 220

TALLAHASSEE. FL 32303

WHITTON, JOHN C

CHIEF BUREAU OF PETROLEUM INSP FL DEPT OF AGR I \& CONSUMER SVC 3125 CONNER BLVD BLDG \#1 TALL AHASSEE FL 32399

\section{HRIGHTE LOU}

WEIGHTS E MEASURES $\mathrm{CS}$

3125 CONNER BLVD

TALLAHASSEE, FL 32301

YOUNG. GENE

LABORATORY MANAGER

FLA DEPT OF AGRIC \& C S

PO BOX 21187 IAUDEROAL, FL 33335 
ZEIGLER ROBERT

PETROLEUH INSPECTOR C S
9828 ATH AVE

ORLANDO, FL 32824

2 IELODAVID

WEIGHTS \& MEA SURES \& S

3125 CONNER BLVD

TALLAHASSEE. FL 32301

\section{DADE COUNTY}

ALFCRD NEIL I
INSPEC OOR CDNSUMER PROTECTION
METROPOLITAN DADE COUNTY

MERM

140 H F A GLER ST STE 1605

$305 \frac{1}{375}-4222$

BUGBEE ROBERT

MENIOR INSPECTOR

MBR

$140 \mathrm{~W}$ FLAGLER ST STE 16.05

MI AM $1, F$

DALEY, ALAN

CON SUMER INSPECTOR

METRO-DADE COUNTY

140 HLAGLER ST STE 1605

$305 \frac{1}{3} 75-4222130$

MBR

HER SHBEIN, ARTHUR

OIRECTOR - DADE COUNTY FLORIDA CONSUMER PROTECTIDN DIVISION

140 W FLAGLER ST/STE 1605

$305375-4222$

MOSLEY FREDDY

INSPECTCR CONSUMER PROTECTION METRO-DADE CONSUAER PRCT DIV

140 FLAGER ST RM 1605

MI AM T FL 33130

SAULS II PRINCE LL PROTECTION

DADE COUNTY CONSUMER PROT DIV

140 W FAGLER ST STE 1605

MIAMI FL 3130

$3053\}_{5}-4222$

SKALKOW SKIE STAN W

METRO-DADE COUNTY CONSUMER PRO

$140 \mathrm{H}$ FLAGLER ST STE 1605

$305 \frac{1}{3} 75-4222$

CITY: SUNRISE

SILUERMAN MELYIN

CITY OF SUNRISE

$10770 \mathrm{~W}$ OAKLAND PARK BLVD

SUNRISE. FL 33321

RG IA

STATE

BOWEN. VERNON

FIELD SUPERVISOR NORTH GA

FUEL \& MEAS DIV/ST DEPT OF AG

AGRIC BLDG CAPITOL SO

ATLANTA: GA 30334

406 656-3605

MBR

MBR

MBR

MER
COILE MARTIN T

DIRECTOR OF WEIGHTS \& MEASURES 作

ATLANTA FARMERS MARKET

FOREST PARK, GA 30050

$404363-7611$

FARLEY: RENEE L

PRINCIPAL LAB TECHNICIAN

WEIGHTS \& MEASURES LAE

ATLANTA FARMER S MARKET

FOREST PARK. GA $30 \mathrm{C} 5 \mathrm{C}$

404 363-7611

GARLAND, DON

ASSISTANT DIRECTOR

STATE FUEL OIL LABCRATORY

5235 KENNEDY RD

FOREST PARK, GA 30050

$404363-7597$

GRIFFIN BERNIE E

FIELD SUPPERVISOR SOUTH GA AGRIC BLOG/RM $327 / C A P I T O L$ SO

ATLANTAO GA 30334

MBR

NCWM

MULLINAX OLIN D

ASSISTAN COMMISSIONER

DEPT OF AGRI FUEL E MEAS DIV

ATLANTA. GA 30334

404 656-3605

TRUBY BILLLEL MEASURES OIV

GA DEPT OF AGR ICUL TURE

AGR I BLDG CAPITAL SO

ATLANTA. GA 30334

404 656-3605

WHITENER BRENDA

LABORATORY SCIENT IST

WEIGHTS 6 MEASURES LABORATORY

ATLANTA FARMERS MARKET

FOREST PARK GA 30050

$404363-7611$

HILLIAMS. CURTIS

FUEL OIL CHEMI ST

GA DEPT CF AGR IC

TEANEOY RD

$404363-7597$

GUAM

\section{STATE}

BENAVENTE, FRANK C

WEIGHTS \& MEASURES INSPECTOR

P 0 BOX 2796

AGANA GUAM. GU 96910

$671472-8981 \times 265$

MBR

SANTOS. DAVE J

DIRECTOR DEPT REVENUE \& TAXES

MBR GOVERNMENT OF GUAM

MBR

NCWM

855 W MARINE DR

AGANA, GU 969

UMP INGO JUAN REYES

SUPERVISQR-WEIGHTS 6 MEASURES GUAM DEPT REVENUE / TAXA TION $P$ O BOX 3663

AGANA GUAM. GU 96910 


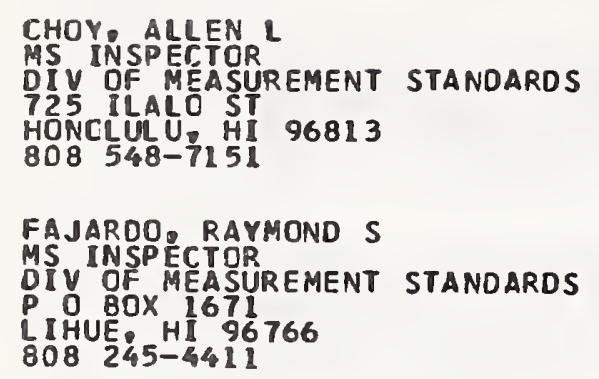

GAMULO, DWIGHT M M MS INSP ECTOR
OIV OF MEASUREMENT STANDARDS
P O BOX 700 HA ILUKI. HI 96793

IMAMURA ROBERT S CHIEF COMM \& TRADE PRACTICES DIV OF MEASUREMENT STANDARDS 725 ILALD ST

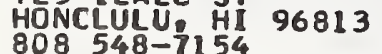

KANESHIRO GARY H MS INSP ECTOR OIV OF MEA SUREMENT STANDAROS 725 ILALO ST HONOLULU, HI
80858813

KUNIYUK I. MICHAEL $K$ MS INSPECTOR HONNOLULU. ST 96813 $808548-7151$

MAKA, JAMES E

CHIEF STANDARDS $E$ TECH SVCS DIV OF MEA SUREMENT STANDARDS 725 ILALO ST HONCLULU. HI 96813

MATTIMOE, GEORGE E ADMINISTRATOR VEASUREMENT STDS NBR 725 ILALOST HONCLULU HI 96813

MIYAKE, ROBERT

MS INSPECTOR

DIV OF MEASUREMENT STANDARDS

P. BOX 1477

HILO HI 96720
ODA. TOM F

MS INSPECTOR

DIV OF MEA SUREMENT STANDARDS

725 ILALO ST

HONOLULU: HI 96813

PAYANAL EARL H

MS INSPECCTCR

DIV OF MEASUREMENT STANDARDS 725 ILALO ST

HONOLULU HI 96813

YAMAMOTO DIANNE N

DIV OF MEASUREMENT STANDARDS 725 ILALO ST HONOLULU

YAZAWA, LESTER T METROLOGIST

DIV OF MEASUREMENT STANDARDS

725 ILALO ST HONOL ULU, HI 96813

I DAHO

STATE

BROOKS. BRUCE

BNSPECTOR - DI STRICT 3-A 2216 KELLOGG LANE

BOISE: ID 83712

$208334-2345$

GALANTUOMINI DAVE

INSPECTOR DISTRICT 4-B

BUREAU OF W्WEIGHTS AND MEASURES

LEWISTON: ID 83501

HARTRUFT MIKE

INSPECTOR OISTRICT 2-B

BUREAU OF WEIGHTS ANO MEASURES ROUTE 1 BOX 1105

PAUL ID 83334

HURDE DALE

BUREAU OF WEIGHTS AND MEASURES BOISE. ID 83702

$208334-2345$

JEX, GLEN

INSPECTOR OISTRICT I-A

BUREAU OF HEIGHTS AND MEASURES

2216 KELLOGG LANE

$208334-234512$

MBR

NCW

MANN. HUOSON P

INSPECTOR

BUREAU OF WEIGHTS 6 MEASURES

HAYDEN LAKE, ID 83835
$208772-9494$ 
MERRITT KEVIN

BUREAU OF WEIGHTS AND MEASURES ROUTE 1

FILER ID 83328

OSWALD WESLEY $S$

INSPECTOR

WE IGHTS E MEA SURES

22 IE KELLOGG LANE

$208334-2345$

PROCTOR, MIKE

INSPECTOR DISTRICT 3-B

BUREAU OF WE IGH

POCATELLO ID 83201

$208232-8270$

SCHAFER , TOM $W$

METROLOGIST

WE I GHTS $E$ MEA SURES

22 IE KELLOGG LANE

BOI SE ID 83712

IS

\section{TATE}

ALLEN, JAN

INSPECTCR AGRIC BLDG/STATE FAIRGROUNDS SPR INGFIELD IL 62706

$217782-3817$

BAINES PAUL PALS MEASURES

ILLINOIS DEPT OFF AGRIC

AGRIC BLOG/STATE FAIRGROUNOS

SPRINGF IELD IL 62706

BAUER PAUL

ILLINOIS DEPT OF AGRICULTURE AGRIC BLDG /STATE FAIRGROUNDS

SPR INGF IELD IL 62706

$217782-3817$

BEHRNS HAYNE W

BUR CHIEF PROD INSP E STANDARD

MBR

STATE FAIRGRCUNDS PO BOX 4906

SPRINGFIELD? IL 62708

BEIERMAN 8ILL

INSPECTOR WTS \& MEA SURES

ILLINOIS DEPT OOF AGRIC

AGRIC BLDG/STATE FAIRGROUNDS

SPRINGFIELD IL 62706

$217782-3817$

BENDELES

INSPECTOR WTS E MEASURES

ILLINDIS DEPT DF AGRIC

SPR INGF IELD, IL 62706

21.7782-3817

BERG RCN

INSPECTOR

ILLINOIS DEPT OF AGRICULTURE

AGR IC BLDG/STATE FAIRGRDUNDS

SPRINGFIELDQ IL 62706

MBR

MBR

MBR

NCWM

NCWM
BERG. STEHART

INSPECTOR WTS \& MEASURES

ILLINOIS DEPT OF AGRIC

AGRIC BLDG/STATE FAIRGROUNDS SPRINGFI ELDE IL 62706

NCWM

COLBOK SIDNEY A

W $\&$ PROGRAM MANAGER

ILLINOIS DEPT OF AGR ICULTURE 801 EAST SANGAMON AVENUE SPR ING FIELD。IL $627 \mathrm{C6}$ $217782-3817$

COWAN PAT

INSPECTOR HTS \& MEASURES

ILLINOIS DEPT OF AGRIC

AGR IC BLDG/STATE FAIRGRCUNDS SPRINGFIELDE IL 62706

$217782-3817$

DIXON, DALE

INSPECTOR WTS 6 MEASURES

ILLINOIS DEPT OF AGR IC

AGRIC BLDG/STATE FAIRGRCUNDS SPRINGFIELD. IL 62706 $217782-3817$

ESTES, DAL WTS \& MEASURES

ILLINOIS DEPT OF AGRIC

AGRIC BLDG/STATE FAIRGROUNDS

SPR I NGFIELD, IL 62706

$217782-3817$
MBR

NCWM 
FORC. DELBERT

INSPECTOR WTS \& MEASURES

ILLINOI S DEPT DF AGRIC

AGR IC BLDG/STATE FAIRGROUNDS SPRINGFIELD: IL 62706

$217782-3817$

GRAY LYLE

INSPECTOR WTS \& MEASURES

ILLINOIS DEPT OF AGRIC SPR INGFIELD: IL 62706 $217782-3817$

GUIN STAN

INSPECTOR HTS E MEASURES

ILLINOIS DEPT OF AGRIC SPRINGFIELD, IL 62706

$217782-3817$

HARPSTRITE . HOHARD

INSPECTOR hTS \& MEASURES

ILLIINOIS DEPT DF AGRIC

SPRINGFIELD, IL 62706

$217-782-3817$

HENRY GENE

INSPECTOR

ILLINOIS DEPT OF AGRICULTURE

AGRIC BLDG/STATE FAIRGRDUNDS

SPR INGFIELD, IL 62706

$217782-3817$

JONES, PAUL

INSPECTOR WTS \& MEASURES

ILLINOIS DEPT OOF AGRIC

AGRIC QLDG/STATE FAIRGROUNDS

SPRINGFIELD: IL 62706

$217782-3817$

KERN GLEN

INSPECTOR WTS E MEA SURES

ILLINDIS DEPT OF AGRIC

AGRIC BLDG/STATE FAIRGROUNDS

SPRINGFIELD, IL 62706

$217782-3817$

LAR SEN KENT
INSPECTCR INSPECTCR
IILINOIS DEPT OF AGRICULTURE
AGRIC BLOG STATE FAIRGROUNDS SPRINGF IELD, IL 62706

217 782-3817

MARKLEY TED

INSPECTOR HTS $\&$ MEASURES

ILLINOIS DEPT OF AGRIC

SPRINGFIELD IL 62706

MATHRE, RICHARO

INSPECJOR

ILLINOI S DEPT OF AGRICULTURE AGRIC BLOG/STATE FAIRGROUNDS SPR INGFIELDO IL 62706 $217782-3817$

MBR

MCGUIRE, STEPHEN E

METRCLOGIST
ILLINDIS DEPT OF AGRICULTURE
801 EAST SANGAMON AVENUE

NCWM

SPRINGFIELD。IL 62706

$217782-3817$

MCLASKEY MARTHA

INSPECTOR WTS G MEASURES

ILLINOI S DEPT DF AGRIC

AGR IC BLDG/STATE FAIRGRQUNDS

SPRINGFIELD: IL 62706

$217782-3817$
MORR I SON. JIM

INSPECTOR WTS E MEASURES

ILLINOIS DEPT OF AGRIC

SPRINGFIELD: IL $627 \mathrm{C6}$

$217782-3817$

MURDENT NORMAN

INSPECTOR WTS E MEASURES

ILLINOIS DEPT OF AGR IC

AGR IC BLDG/STATE FAIRGRCUNDS

SPR INGFIELD: IL $627 \mathrm{C} 6$

$217782-3817$

MURPHY DAVE

INSPECTOR HJS 6 MEASURES

ILLINOIS DEPT OF AGRIC

AGRIC BLDG/STATE FAIRGRCUNDS

SPR INGFIELD. IL 62706

$217782-3817$

OZEE C $K$

SECOND DIVISION VERICLE SECT 201 EAST ADAMS SUITE 250

SPR INGFIELD. IL 62 C 71

$217782-0504$

PHILMON RICHARD L

ILL INOIS DEPT OF AGR IC

AGRIC BLDG/STATE FAIRGR

$217782-3817$

PLYM. GREG

INSPECTOR HTS \& MEASURES

ILL INOIS DEPT OF AGRIC

AGRIC BLDG/STATE FAIRGRCUNDS SPR INGFIELD. IL $627 \mathrm{C6}$

$217782-3817$

ROMANO. PAUL.

INSPECTOR WTS 6 MEASURES

ILLINOIS DEPT OF AGRIC

AGRIC BLDG/STATE FAIRGRGUNDS

SPRINGFIELD!IL 62706

$217782-3817$

SCHELLER, GENE

INSPECTOR WTS E MEASURES

ILLINOIS DEPT OF AGRIC

AGR IC BLOG/STATE FAIRGROUNDS SPRINGFIELD。IL $627 \mathrm{c6}$

$217782-3817$

SHOEMAKER DENNIS

INSPECTOR WTS \& MEASURES

ILLINOIS DEPT OF AGRIC

AGRIC BLDG/STATE FAIRGRCUNDS

SPRINGFIELD: IL 62706

SHORT:BILL

ILL INOIS DEPT OF AGRIC

AGR IC BLDG/STATE FAIRGROUNDS

SPRINGFI ELD: IL 62706

$217782-3817$

SHROYER \& TERRY

INSPECTOR WTS E MEASURES

ILLINOIS DEPT OF AGRIC

AGR IC BLCG/STATE FAIRGROUNDS

SPR INGFIELDDIL $627 \mathrm{C6}$

$217782-3817$

STE IN. GER ALD

INSPECTOR

ILLINOIS DEPT OF AGRICULTURE AGR IC BLDG/STATE FAIRGRCUNDS SPRINGFIELD:IL $627 \mathrm{C} 6$

$217782-3817$ 


\section{ITY: BERHYN}

SLACEK. FRANK

CITY SEALER

CITY DF BERHYN

BERhYN, IL 60402

$312788-2660$

IITY: CHICAGO

BLACKMON JJESSE

CITY OF CHICAGO

$121 \mathrm{~N}$ LASALLE RM $80 \mathrm{~B}$

CHICAGO IL 60602

$312744-4092$

MADISON JESSE D

COMN ISS IONER

DEPT OF CONSUMER SERVICES

$121 \mathrm{~N}$ LASALLE CITY HALL

C HICAGD. IL 60602

$312744-4090$

CITY: CICERD

STASIOR TED

CITY OF CICERO

4932 W 25 PLACE

$312656-3600$

CITY: DES PLAINES

HINDE ROBERT E

CIIY SEALER

CI TY OF DES PLAINES

1420 MINER ST ROOM 401

DES PLAINES IL 60016

CITY: EVANSTON

RUSS JR, JOHN P

WE IGHTS $\&$ MEASURES LICENSING

CITY OF EVANSTON

$210 O R I D G E$ AVE

EVANSTON IL 60204

$312328-2100 \times 2256$

CITY: GRANITE CITY

DESCUSSE, ERVIN

INSPECTIÓN DEPT

CITY OF GRANITE CITY

2301 ADAMS ST

GRANITE CITY.

IL 62040

HEBB WILBURN

INSPECT ION DEPT

CIIY OF GRANITE CITY

2301 ADAMS ST

$618452-6225^{\circ}$

IL 62040

CITY: HARVEY

JONES ROBERT C

CITY SEALER E HEALTH INSPECTOR CI TY OF HARVEY PUBL IC AFFAIRS

15320 BROADWAY PO BCX 617

HARVEY 11060426
$312339-4200 \times 29$

MBR

MBR

MBR

\section{CITY: OAK PARK}

CASSEN VIRG INIA

CITY SEALER

VARK

I

312 383-6400

CITY: RIVER GROVE

GUARINOD PAUL

CIIY SEALER

CITY OF RIVER GROVE

2621 N THA TCHER AVE

RIVER GROVE IL 60171

NCWM

CITY: ROCKFORD

MARTINE HILLIAM A

CITY SEALER

CITY OF ROCKFDRD

425 E STATE ST ROON 706

R15KF 987-55 50

CITY: SCHAUMBURG

SALERNO. MARTINM

LICENSE AND PERMIT OFFICER

VILLAGE OF SCHAUMBURG

101 SCHAUMBURG CT

SCHAUMBURG. IL 60193

NCHM

CITY: SKOKIE

ANDERSON. LARRY $R$

HEIGHTS \& MEASURE INSPECTOR

VILLAGE OF SKOKIE

5127 OAKTON STREET

$312673-0500 \times 244$

MBR

NCHM

NCWM

INDIANA

STATE

BAXTER, BRUCE A

INSPECTOR

DIVISION OF WEIGHTS G MEASURES

1330 WEST MICHIGAN ST

INDIANAPOLIS. IN 4 E206

$317633-0350^{\circ}$

BOYKIN, PETER H

METROLOG IST

DIVISION OF WEIGHTS $\&$ MEASURES

1330 HEST MICHIGAN ST

INDIANAPCL IS. IN 46206

CLINGAMAN JR JERRY L 2622 FREDONIA RD

INDIANAPOLIS. IN 42222

$317633-0350^{\circ}$

DECAMP ROBERT C

INSPECTOR

DIVISION OF WEIGHTS MEASURES

1330 HEST MICHIGAN ST

INDIANAPOL IS. IN 46206

$317633-0350^{\circ}$
MBR

MBR

NCWM 
FINNEY RALPH

INSPECYOR

DIVISION OF WEIGHTS \& MEASURES

1330 HE ST MICHIGAN ST

INDIANAPQLIS. IN 46206

GANDY DONALD W

INSPECTOR

OIVISION OF WEIGHTS \& MEASURES

1330 WEST MICHIGAN ST

$317633-0350$

HORAN MICHAEL A

INSPECTOR

DIVISION OF HEIGHTS 8 MEASURES

1330 MEST MICHIGAN ST

INDIANAPQLIS.
$317633-0350^{\circ}$

QUAKENBUSH. TIMOTHY A

INSPECTCR

DIVISION OF WEIGHTS 6 MEASURES

1330 WEST MICHIGAN ST

INDIANAPOLIS. IN 46206

\section{RHOADES SHARON}

DIVISION OF HEIGHTS $\&$ MEASURES

1330 MICHIGAN ST

INDIANAPOLIS. IN 46206

\section{SEITZZ HENRY PAUL}

INSPECTCR

DIVISION DF WEIGHTS 6 MEASURES

INDIANAPOLIS. IN 46206

$317633-0350^{\circ}$

SKINNER SEDRICK E

DIVISION OF WEIGHTS $E$ MEASURES

$133 \mathrm{C}$ WEST MICHIGAN ST

INDIANAPOLIS. IN 46206

STUMP LAWRENCE J

INSPECTOR

DIVISION OF WEIGHTS $\&$ MEASURES

1330 WE ST MICHIGAN ST

INDIANAPCLIS.

THOMPSON MORRIS

IN STATE WEIGHTS/MEASURES

1330 MICHIGAN ST

INDIANAPOLIS. IN 46206

WALKER, ROBERT H

DIRECTOR

WEIGHTS AND MEASURES DIVISION

1330 W MICHIGAN ST

INDIANAPOLIS. IN 46206

\section{BARTHOLOMEW COUNTY} GR ITZER CHARLES F DEPT OF WEIGHTS \& MEASURES CNTY OFF BL-RM 305 440-3RD ST COLUNBUS IN 47201

MBR

NCWM

MBR

NCWM

\section{CASS COUNTY}

YEAKLEY LEE WAYNE

INSPECTOR WEIGHTS MEASURES

CASS COUNTY

200 CDURT PARK ROON 312

LOGANSPORT IN 46947

$219722-5050 \times 223$

CLARK COUNTY

BRADSHAW, HAROLD D

INSPECTOR/WEIGHTS EMEASURES

DEPT OF WEIGHT AND MEASURES

CITY COUNTY BLDG RCCM 314

JEFFERSONVILLE. IN 47130

$812283-4451 \times 620$

\section{CLINTON COUNTY}

SURFACE , ROY

CLINTON COUNTY WEIGHTS \& MEAS 252 FAIRGRDUND ST

FRANKFORT: IN 46041

DEKALB COUNTY

BASSETT: VIRGIL

INSPECTOR

DEPT DF WEIGHTS 6 MEASURES

509 S MAIN ST

AUBURN. IN 46706

219 925-2316

DELAWARE COUNTY

BITTINGER . HERB

DELAWARE COUNTY WGIS AND MSRS

COUNTY BUILDING ROCM 208

MUNCIE. IN 47305

$317747-7714$

SATOM. WILLIAM

DEPUTY INSPECTOR

DELAWARE COUNTY HTS $\&$ MEAS

ROOM 208 COUNTY BUILDING

MUNCIE. IN 47302

$317747-7714$

ELKHART COUNTY

COOK BILL D

INSPECTOR

ELKHART COUNTY WEIGHTS 8 MEAS

58440 VALLEY VIEW CRIVE

$219293-2228$. 46517

$M B$

NC

FLOYD COUNTY

MOREILLON JAMES M

NEW ÁLBANY-FLOYD CC

1203 WESTHCOD LANE

NEW ALBANY IN 47150

FULTON COUNTY

ST CLAIR, DEVON A

WEIGHTS AND MEASURES INSPECTCR

RR 2

KEWANNA. IN 46939

$219857-3683$
MBR

NC WP

BR

NCWN

MBR

$\mathrm{NCh}$

in

BR

NCWM 
SEVIER: WILLIAM R

WEIGHTS AND MEASURES INSPECTOR 800 S PRINCE

PRIACETCN IN 47670

$812385-2426$

RANT COUNTY

SIMPKINS, CURTIS E INSPECTOR GR ANT COUNTY

401 SOUTH ADAMS ST

MARION: IN 46952
$317668-8871 \times 170$

IANCOCK CCUNTY

LOWE ROBERT $P$ INSPECTOR WEIGHTS \& MEASURES HANCOCK COUNTY INDI ANA

RR 2 BOX 98

NEH PALESTINE. IN 46163
$317861-4158$

HENDRICKS COUNTY

HUGHEY, CLAYTON H HUGHEY CLAYTON H
INSPEC OOR OF WEIIGHT \& MEASURE NBR
HENORICKS COUNTY

3421 IND IANA RD

PLAINFIELD IN 46168

HOWARD COUNTY

DAILY ROBERT J

INSPEETOR OF WEIGHTS \& MEASURES

100 S UNION-CITY HALL-3RD FL

KOKOMO IN 46901

$317456-7467$

GIST GARY R

INSPECTCR

DIVISION OF HEIGHTS \& MEASURES 100 S UNION-CITY HALL-3RD FL KOKOMO IN 46901

$317456-7468$

HUNT INGTON COUNTY

ENDSLEY ROBERT D

IN SPECTOR

WEIGHTS E MEASURES

313 WARREN ST

HUNTING TON IN 46750

219 356-3616

JACKSCN COUNTY

BREWER, ROBERT J

INSPECTOR

DIVISION OF WEIGHTS \& MEASURES

RR 1 BOX 88A

VALLONIA. IN 47281

\section{JEFFERSON COUNTY}

ALEXANDER, A B

IN SPECTOR

DIVISION OF HEIGHTS $\&$ MEASURES

348 BELLAIRE DR

MAD ISON. IN 47250

$812273-4439$

MBR

NCHM

MBR

NCWM

MBR

MBR

NCWM
HANDY WAYNE E

INSPECTOR

HEIGHTS AND MEASURES

JOHNSON COUNTY COURTHOUSE

FRANKLIN IN 46131

$317736-5000 \times 209$

\section{KNOX COUNTY}

INSPECTOR

DIVISION OF WEIGHTS $\&$ MEASURES

RR \#3 BOX 632

HOWE IN 46746

$219562-2160$

LAKE COUNTY

MYSOGLAND, ALBERT

COUNTY SEALER

WEIGHTS AND MEASURES

$2293 \mathrm{~N}$ MAIN ST

CROWN POINT. IN 46307

$219738-2020 \times 301$

\section{LAPORTE COUNTY}

HANISH EDWIN M

INDIANA WEIGHTS \& MEASURES

2700 FRANKLIN ST

MICHIGAN CITY. IN 46350

$219874-7197$

LAWRENCE COUNTY

ANDERSON, GENE

INSPECTOR

DIVISION OF WEIGHTS 8 MEASURES P O BOX 233

BEDFORD. IN 47421

$812275-7306$

MADISON COUNTY

MOORE. CHARLES $W$

COUNTY INSPECTOR

WEIGHTS \& MEASURES OF MADISON

MADISON COUNTY GOVT CENTER

ANDERSON, IN 46011

$317646-9247 \times 18$

MARSHALL COUNTY

GRA YERSON. WILLIAM

INSPECTOR

DIVISION OF WEIGHTS \& MEASURES

436 E NORTH ST

BREMEN. IN 46506

$219546-4403$

MBR

NCWM

MBR

NChM

MBR

MBR

NCWM

MBR

NCWM

MIAMI COUNTY

PERSONETT. MERRILL $P$

MIAMI CNTY INSPECTCR WTS MEAS SI I GHTS

PERU IN 46970

$317473-3319$
MBR

NCWM 
HEADLEY IVAN E

CHIEF INSPECTOR

MONRCE CNTY WEIGHTS 6 MEASURES

MBR

$119 \mathrm{H} 7 \mathrm{TH}$ ST

BLOCMINGTON, IN 47401

MONTGGMERY COUNTY

JOHNSON FRANKL IN B

MONTGOHERY CO INSPECTOR HEM

CRAHFORDSVILLE, IN 47933
$317866-0306$

NOBLE COUNTY

BUCHANAN ARTHUR

COURTHOUSE 1 ST FLOOR

ALBION. IN 46701

PORTER COUNTY

CLAUSSEN. RICHARD H

DIRECTOR RM 105

PORTER CCUNTY HEIGHTS $\&$ MEAS

CDURTHOUSE ANNEX 140 IN CALUMET

YALPARAISO. IN 46383

ST JOSEPH COUNTY

KLEIN。 PAUL J

INSPECTOR WEIGHTS \& MEASURES

ST JOSEPH CDUNTY

227 WEST JEFFER SON BLVD

$219284-9751$

TIPPECANOE COUNTY

YANDERHIELEN.JAMES

INSPECTOR

TIPPECANOE CNTY HEIGHTS 2 MEAS CON THIRD ST/BOX 347

LAFAYETTE IN

VANDERBURGH COUNTY

TOWNSEND, LORETTA

CHIEF INSPECTOR-CITY E COUNTY

WEIGHTS \& MEASURES DEPARTMENT

COMMUNITY CENTER 100 E WALNUT

$812428-0623$ IN 47708

VIGO COUNTY

SCOTT JAMES A

INSPECTOR

WEIGHTS AND MEASURES VIGO CO

ROGN 5 CCURTHOUSE

TERRE HAUTE, IN 47801

HABASH COUNTY

OUREIN. ROBERT $C$

WABASH COUNYY INSSPECTOR

$1911 / 2$ SOUTH HABASH

WABASHO IN 46992

MBR

MBR.

MBR

NCWM

MBR

NCHM

MBR

NCWH

NCHM

NCWH
RAVEN WILLIAM

1049 NORTHWOOO OR

BOONYILLE $897-5772$ IN 47601

CITY: ANDERSON

LAND. ROBERT L

INSPECTOR DF CITY ANDERSON

DEPT OF HEIGHTS E MEASŨRES

PO BOX 2100120 E 12 TH ST

ANDERSON IN 46011

$317646-9839$

CITY: EAST CHICAGO

SZYNDROWSKI CHESTER

1102 WEST $1515 T$ ST

$219397-3409^{\circ}$ IN 4 EOII

CITY: FORT WAYNE

PEARSON, REGINALO T

DIRECTOR FORT HAYNE BRANCH

WEIGHTS \& MEASURES DEPTMENT 19 E MAIN ST

FORT HAYNE IN 46801

CITY: GARY

MCCRADY ROBERT
INSPECTORR

DIVISION OF WEIGHTS \& MEASURES

CITY HALL 1100 MASS ST

$219886-0145$

CITY: HAMMOND

BRAHOS DEAN

CITY SEALER

HEIGHTS AND MEASURES DEPT

649 CONKEY ST

HAMMOND: IN 46324

CITY: INDIANAPOLIS

BLAND. RCNALD

DEPUTY INSPECTOR

DEPT OF WEIGHTS $\&$ MEASURES

CITY COUNTY BLDG RCCM I760

INDIANAPOLIS. IN 46204

HANNAH RALPH

DEPUTY INSPECTOR

DEPT OF WE IGHTS $\&$ MEASURES

CITY COUNTY BLDG RCOA 1760

INDIANAPOLIS. IN 46204

NBC

NE

JONES SCOTT

CIYYCIOA

C INDIANAPOL IS

INDIANANTY BLOG RCCM 1760

$317236-4272$

MBR

LOHE ROBERT E

DEPT OF HECTOR

DEPT OF WE IGHTS \& MEAS URES

ROOM 1760 CITY-COUNTY BLDG

INDIANAPOL IS, IN 46204 
PAPPAS GUS A

ADMINIS TRATOR

ROOM 1760 CITY-COUNTY BLDG

INDI ANAPOLIS. IN 46204

$317236-4272$

PHILLIPS, MICHELLE

DEPUTY INSPECTOR \& MEASURES

CITY COUNTY BLDG ROCM 1760

INDIANAPOL IS: IN 46204

$317236-4272$

PIRLOT ALLEN

DI IISION OF WEIGHTS 6 MEASURES

CITY COUNTY BLDG ROOM 1760

INDIANAPCLIS. IN 46204

$317236-4272$

\section{WISEE DCNALO}

DIYISION OF WEIGHTS $E$ MEASURES

CITY-COUNTY BLDE RDOM 1760

INDIANAPOLIS. IN 46204

$317236-4272$

ITY: MI SHAWAKA

STAFFELDT GEORGE $W$

CITY SEALER MEASURES

CITY HALL

MISHAWAKA, IN $\mathbf{4 6 5 4 4}$

219 258-1622

CITY: NEW CASTLE

HYDEN, GERALD L

INSPECTOR

DIVISION OF WEIGHTS $\&$ MEASURES

227 N MAIN ST

NEW CASTLE IN 46362

CITY: RICHAONO

LEWIS DANA

CITY OF RICHMOND

CITY BLDG/50 NORTH FIFTH ST

RICHMOND 317 IN

CITY: SOUTH BEND

NAGY JCSEPH

SEALER OF WEIGHTS $\&$ MEASURES

CITY OF SOUTH BEN

SOUTH BEND IN 46621

$219284-9273$

CITY: TERRE HAUTE

THCMAS, LARRY

CHIEF INSPECTOR

CITY OF TERRE HAUTE

3010 NORTH 5 TH ST

TERRE HAUTE IN 47807

812 232-4767

MBR

MBR

NCHH

MBR

MBR

STATE

BROWN RCBERT $K$

LAB ASS ISTANT II

IDWA DEPT AGRICULTURE

HENRY A WALLACE BLDG

DES MOINES:
HOLLIS: ROBERT E

EIGHTS $\&$ MEASURES

H A HALLACE BLDG

DES MOINES IA 50319

$515281-5861$

DCONNOR, JAMES $M$

SUP ERY ISOR

HEIGHTS AND MEASURES

HENRY A WALLACE BLCG

DES MOINES IA 50319

WAKEFIELD\& JAKE PRCDUCTS

IOWA DEPT AGRICULTURE

H A WALLACE BUILOING

DES MOINES IA 50319

$515 \quad 281-3545$

KANSAS

STATE

AKEY: JAMES ${ }^{H}$

KANSAS WEIGHTS \& MEASURES LAB

2016 SH 37 TH ST

TOPEKA0 KS 66611

MBR

NCWM

DECKER MBR

DECKER ERANK ${ }^{M}$
LP GAS INSPECTOR

KANSAS WEIGHTS ANO MEASURES

731 LNCOLN STREET

EMPOR IA. KS 66801

$913267-4641$

DIETZ. HAROLD

AG INSP ECTOR

KANSAS WEIGHTS G MEASURES

2058 COLLEGE HEIGHTS RD

MANHATTAN: KS 66502

$913267-4641$

FRIEZE: CHARLES

AG INSPECTOR I

KANSAS WEIGHTS \& MEASURES

1420 COOLIDGE

WCHITA. KS 67203

$913267-4641$

GRIZZLE Y IRGIL

AG INSPECTOR I \& MEASURES

618 S WESTERN

CHANUTE KS 66720

$913267-4641$

NCWM

HURSTE ARCHIE SEALER \& DIRECTCR

MBR

KANSAS WEIGHTS E MEASURES

2016 SH 37 TH ST

$913267-4641$

MUS ILO CURT

AG INSPECTOR

KANSAS WEIGHTS \& MEASURES

$B O X 183$

BREWSTER KS 67732

$913267-4641$

PIERCE, RONALD

AG INSPECTOR I \& MEASURES

MBR

SLITH ST

MCRM

MBR

NCWM

$913267-4641$ 
SPRANG FRANCIS

KANSAS HEIGHTS \& MEASURES

1907 FISHER/RR 1 BOX 46

VALLEY FALLS. KS 66088

913 267-4641

STARR \& KEITH D

AGR ICULTURE INSPECTOR II

KANSAS WEIGHTS \& MEASURES

2016 SW 37 TH ST

TPKA? KS 66611

SWAFFORD, DALE W

PETROLEUH MEASUREMENT INSP

STATE OF KANSAS

1417 STARKEY

AUGUSTA: KS 67010

WALTHAN E WILLIAM

AG INSPECTOR ISION OF WEIGHTS \& MEASURES

MBR

PO BOX 87

PAXICO, KS 66526

913 267-4641

HILKE RENNETH $M$

CHIEF COUNSEL LEGAL DIVISION

KANSAS GDARD OF AGRICULTURE

109 SW STH ST

TOPEKA, KS 66612

$913296-3848$

CITY: KANSAS CITY

GUNJA ROBERT

STANDARDS INSPECTOR

CITY OF KANSAS C

KANSAS CITY KS 66101

KINCAID BRENDA $K$

STANDARDS INSPECTOR

CITY OF KANSAS CITY

TOI NORTH 7TH ST

KANSAS CITY KS 66101

LYNCH, DCNALD L

STANDAR DS ADMINIISTRATOR

CITY OF KANSAS CITY

TOIN 7 TH STREE

KANSAS CITY KS 66101

CITY: TOPEKA

WE ICK DONALD J

CITY SEALER

$320 \%$ CRANE

TOPEKA: KS 66603

$913235-9630$

KENTUCKY

STATE

BAIRD, TOAMY

DIV OF HEIGHTS \& MEASURES

106 WEST 2 ND ST

FRANKFORT KY 40601
$502564-4870$

MBR

MBR

MBR
BOYD.JEFFREY

IV WEIGHTS 6 MEASURES

106 WEST 2ND ST

FRANKFORT KY 40601

$502564-4870$

MBR

MBR

MBR

NCWM

NCWM

NCWM

MBR

NCWM

NCWM

DRUEN, AARON

DIV OF WEIGHTS $\&$ MEASURES

106 WEST 2ND ST

RANKFORT. KY 40601

$502564-4870$

ETHINGTON, WAL TER

ROUTE 4

FRANKFORT KY 40601

502 227-7364

FAIRCHILD JUOE S MEASURES

106 WEST 2ND ST

FRANKF ORT: KV 40601
$502564-4870$

GEVEDON, GLENN

DIV OF HEIGHTS \& MEASURES

106 WEST 2ND ST

FRANKFDRT KY 40601

$502564-4870$ 
HADDIK. ROY

DIV OF WEIGHTS 6 MEASURES

106 WEST 2ND ST

FRANKFORT:KY 40601

$502564-4870$

HENDERSCN ROBERT

DIV DF WEIGHTS $E$ MEASURES 106 WEST 2ND ST

FRANKFORT KY 40601

$502564-4870$

KELLEY. LARRY

DIV OF WEIGHYS $E$ MEASURES

106 WEST SECOND

FRANKFURT: KY 40601

$502564-4870$

LINKOUS DERL

DIV OF WEIGHTS 6 MEASURES

106 HEST 2 ND ST

FRANKFORT $\mathrm{KY} 40601$
$502564-4870$

MASSEY, ROY

DIY OF WEIGHJS $C$ MEASURES

106 WEST 2ND ST

FRANKFORT KY 0601

$502564-4870$

MOLEN JAHES P

HEYGHTS 6 MEASURES

106 WEST 2 ND ST

FRANKFORT KY 40601
$502564-4870$

MONTELL CHARLES

DIV DF WEIGHTS E MEASURES

FRANKFORT. KY 40601

$502564-4870$

NALL. LUTHER

DIV OF WEIGHTS \& MEASURES

106 WEST 2ND ST

FRANKFORT: KY 40601

$502564-4870$

PAGE VICTOR

SUPERVIISOR

DIV OF WTS AND MEAS AGRICULTUR

106 WEST 2ND ST

FRANKFORT: KY 40601

$502564-4870$

PEGUES, CAVID

DIV OF WEIGHTS \& MEASURES

106 WEST 2ND ST

FRANKFDRT KY 40601

$502564-4870$

PORTER D JAMES E

DIY OF WEIGHTS E MEASURES

106 WEST 2 ND ST

FRANKFORT KY 40601

$502564-4870$

PREBBLE. CHARLES L

DIRECTOR OF WEIGHTS 8 MEASURES

OIVISION OF WEIGHTS E

FRANKFORT KY 40601

$502564-4870$

REEC, WILLIAM W

DIV OF HEIGHTS $\&$ MEASURES

106 WEST 2 ND ST

FRANKFORT KY 40601

$502564-4870$
ROBINSON: DONALD

DIV OF WEIGHTS \& MEASURES

DIV OF WEIGHTS

FRANKFDRT KY 40601

$502564-4870$

SOUTHERLAND, ROGER

DIV OF WEIGHTS \& MEASURES

106 WEST 2 ND ST

FRANKFORT KV 40601

UIVRETTE RICHARD

DIV OF WEIGHTS $\&$ MEASURES

106 WEST 2ND ST

FRANKFORT KY 40601
$502564-4870$

WETHINGTON HERMAN

DIV OF WEIGHTS 6 MEASURES

106 HEST 2 ND ST

FRANKFDRT: KY 40601

$502564-4870$

WHITAKER . MARK L

METROLOGIST

DIV OF WTS AND MEAS AGRICULTUR

106 WEST 2ND ST

FRANKFORT. KY 40601

$502564-4870$

WILLIAMS CHARLIE

DIV OF HEIGHTS 8 MEASURES

106 WEST 2 ND ST

FRANKFORT KY 40601

$502564-4870$

WISE. RANDALL

DIV OF WEIGHTS 6 MEASURES

106 WEST 2 ND ST

FRANKFORT: KY 40601
$502564-4870$

WORTHINGTON. MICHAEL L

DIV OF WEIGHTS E MEASURES

106 WEST 2 ND ST

FRANKFORT KY 40601

$502564-4870$

\section{LOUIS IANA}

STATE

ASHY, LIONEL

DIVISION OF WEIGHTS $E$ MEASURES

LA DEPT OF AGR ICUL TURE

P O BOX 44456 CAPITCL STATION BATON ROUGE LA 70804

$504925-3780$

BASS: W LED WEION OF WE I E MEASURES

LA DEPT OF AGR ICUL TURE

P O BOX 44456 CAPITOL STATION BATON ROUGE LA 70804 $504925-3780$

BLALOCK, CHRIS

DIVISIDN OF WEIGHTS 6 MEASURES LA DEPT OF AGR ICULTURE P O BOX 44456 CAPITOL STATION BATON ROUGE LA 70804 504 925-3780 FAGE J JOHN W LA DEPT OF AGR ICUL TURE P O BOX 44456 CAPITCL STATION BATON ROUGE: LA 70804

$504925-3780$ 
HARRELL RONALO

DIRECTOR OF WTS $\&$ MEASURES

LA CEPT OF AGRICULTURE

BATCN RCUGE CAPITOL STATION

$504925-3780$

HAYESO TERRY

DIVISION OF WEIGHTS $\&$ MEASURES

LA DEPT OF AGRICULTURE

P O BOX 44456 CAPITCL STATION

BATCN ROUGE LA 70804

$504925-3780$

JENKINS, DENTON

DIVISION OF WEIGHTS 6 MEASURES

LA DEPT OF AGRICULTURE

P O BOX 44456 CAPITCL STATION

BATCN ROUGE LA 70804

$504925-3780$

LILES, ROLANO A

DIVISION DF HEIGHTS \& MEASURES

LA DEPT OF AGRICULIURE

P O BOX 44456 CAP TTCL STATION BATCN RCUGE LA 70804

LOFTON CARL

DIVISION OF HEIGHTS 6 MEASURES

LA DEPT OF AGRICULTURE

BATCN 44456 CAPITOL STATION

$504925-3780$

LYONS. HELVIN

ASST DIRECTOR OF WTS E MEAS

LA DEPT OF AGRICULTURE

P O BOX 44456 CAP ITCL STATION

BATCN ROUGE LA 700804

\section{MANDA. LISA}

DIVISION OF WEIGHTS $\&$ MEASURES A DEPT OF AGRICULTURE PO BOX 44456 CAPITOL STATION BATCN ROUGE:
$504925-3780$

MILLICAN. SUSAN

DIVISION OF WEIGHTS 8 MEASURES

LA DEPT OF AGRICULTURE

PO BOX 44456 CAPITOL STATION

BATCN RCUGE: LA 70804

504 925-3780

SHAh BOBBY G

DIVISION OF WEIGHTS E MEASURES

LA DEPT OF AGRICULTURE

P O BOX 44456 CAPITOL STATION

BATCN ROUGE LA 708 C4

SIMMONS: GERALO

OI VISION OF WEIGHTS 6 MEASURES

LA DEPT OF AGRICULTURE

BATON ROUGE LA 70804 STATION

$504925-3780$

SHITH JESSE

DIVISION OF WEIGHTS E MEASURES

LA DEPT OF AGRI CULTURE

$P$ B BOX 44456 CAPITCL STATION

BATCN ROUGE: LA $70 B 04$

WILLIAMS, BENNETT

DI VISION OF WEIGHTS E MEASURES

P O

BATCN ROUGE LA TOBCL STATION

BATCN ROUGE
$504925-3780$
MBR

MAINE

\section{STATE}

AUSTIN口 BERNARO H

SUPERVISOR OF HEIGHTS 8 MEAS

MAINE BUREAU OF HEIGHTS $\&$ MEAS BUREAU OF L $\&$ M STATION 28 AUGUSTA. ME 04333

$207289-3841$

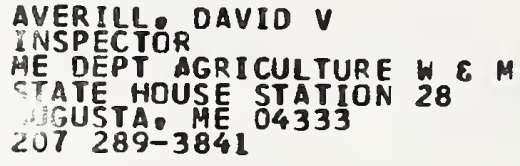

BROWN CONRAO

INSPECTOR SE OEPT AGRICULTURE H ${ }^{6} M$

AUGUSTA ME 207

CUNNINGHAM. JOHN

INSPECTOR

AE DEPT AGRICULTURE I $6 M$

STATE HOUSE STATION 28

AUGUSTA. ME 04333

$207289-3841$

DAVISO CLAYTON F

AGR ICULTURE INSPECIICNS OIV STATE HOUSE STATION 28

AUGUSTA. ME 04333

OQWLING KENNETH E INSPECTOR ME DEPT AGRICUTURE W E M OFFICE BLOG STATION 28 AUGUSTA ME 04333

$207289-3841$

FRENCH, WILLIAM R INSPECTOR

ME OEPT AGRICULTURE \& $\mathrm{M}$ STATE HOUSE STATION 28 AUGUSTA: ME 04333

MILLAY STANLEY $K$

METROLOGIST DEPT AGRICULTURE h $\& M$

OFFICE BLDG STATION 28

AUGUSTA. ME 04333

$207289-2751$

CITY: ALLAGASH

BROWNELL CLIFFORO

SEALER OF UEIGHTS E MEASURES ALLAGASH PLANTATION ALLAGASH. ME 06774

CITY: BAR HARBOR

POIRIER D DUDLEY

SEALER OF WEIGHTS 6 MEASURES CITY OF BAR HARBOR 95 LEOGELAWN AVE BAR HARBOR. ME 04605

CITY: BIDOEFORD

CAMAIRE. PAUL SEALER OF WEIGHTS 6 MEASURES IIDDEFORO SACO BIDOEFORD. ME 04005 
(ITV: BINGHAM

WHI TMAN DON

SEALER CF WEIGHTS \& MEASURES

BI NGHAM MOSCOW

BINGHAM. ME 04920

ITY: BLUE HILL

ROBERTSCN. RAYMOND

BLUE HILL SURRY

MUNICIPAL OFFICE

BLUE HILL. ME 04615

ITY: BRUNSWICK

MARQUIS JR JOHN B MEASURES SEALER OF WEIGHTS E MEASURE 48 PLEA SANT ST

BR.UNSWICK, ME 04011

IITV: CORINNA

2IMMERMAN。 ELMER

SEALER OF WEIGHTS \& MEASURES COR INNA DANFORTH ORIENT

COR INNA. ME 04928

CITY: DEER ISLE

ROBBINS MAURICE SEALER CF WEIGHTS \& MEASURES DEER ISLE. ME 04627

CITY: DEXTER

BOWEN: GLADWYN

SEALER CF WEIGHTS \& MEASURES

CITY OF DEXYER

RAIILOAD AVE

DEXTER. ME 04930

CITY: DOVER FOXCROFT

ANDERSON, ROEERT E

SEALER OF WEIGHTS \& HEASURES CITY OF DOVER FOXCR OFT

DOVER FOXCROFT. ME 04426

\section{CITY: EASTPORT}

DAVIS. MARY

SEALER OF WEICHTS $\varepsilon$ MEASURES

CITY OF EASTPORT

EASTPORT. ME 04631

\section{CITY: FAIRFIELD}

FLEWELLING JAMES R MEASURES 34 TEST ST FAIRFIELD, ME 04937

CITY: GUILFORD

RIQUX: J A MAIN ST

GUILFORD. ME 04443

\section{CITY: HARMONY}

HURD LELAND A

SEALER OF HEIGHTS $\&$ MEASURES CITY OF HARMONY HARMONY. ME 04941

CITY: JONESPORT

PRESLEY CARL

SEALER OF WEIGHTS $\&$ MEASURES JONESPORT. ME 04645

CITY: KINGFIELD

DAVIDSON. L DANA

SEALER OF WEIGHTS \& MEASURES KCHOOL ST

CITY: LIMER ICK

CHAMBERL AIN ALLAN H

SEALER OF WEIGHTS \& MEASURES

CITY OF LIMER ICK

SOKOKIS TRAIL

LIMER ICK. ME 04038

CITY: LINCOLN

HEAVIN. BOBBY

SEALER OF WEIGHTS \& MEASURES

CITY OF LI NCOLN

1 TAYLOR ST

LINCOLN. ME 04457

\section{CITY: LINCOLNVILLE}

HAROY WILLARD

SEALER OF WEIGHTS $\varepsilon$ MEASURES

CITY OF LINCOLNVILLE

LINCOLNVILLE. ME 04849

CITY: MECHANIC FALLS

PATTERSON, DONALO I

SEALER OF WEIGHTS \& MEASURES

CITY DF MECHANIC FALLS

P O BOX $63 / 12$ MAPLE ST
MECHANIC FALLS. ME C4256

\section{CITY: MILLINOCKET}

ANGOTTIDR ICHAR:D

SEALER OF WEIGHTS $\&$ MEASURES

CITV OF MILLINOCKE I

17 VERMONT ST

MILLINOCKET. ME 04462

CITY: MILO

MORRISON PERL RFO

MILD. ME 04463

CITY: NEW GLOUCESTER

JOY GREGORY

SEALER OF WEIGHTS 6 MEASURES NEW GLDUCESTER GRAY RAYMOND RT 1 BOX 267

NEH GLOUCESTER. ME 04260 
BAILEY RODNEY

SEALER OF WEIGHTS $\&$ MEASURES CITY OF HHITEFI ELD

NORTH WHITEFIELD, ME 04353

\section{CITY: RANGELEY}

ELLIS, BRIAN C

SEALER OF WEIGHTS \& MEASURES CITY OF RANGELEY

4 PCND ST BOX 36

RANGELEV, ME 04970

CITY: RUMFORD

CHABOT, RAYMOND

MEALER CF WEIGHTS \& MEASURES MEX ICO RCX SURY

RUMFORO, ME 04276

CITY: SABATTUS

HODSDON FRANK R CITY OF SABATTUS

SABATTUS, HE $04280^{2}$

CITY: SARGENTVILLE

WARCWELL JR HORACE

SEALER OF WEIGHTS \& MEASURES

SARGENTV SEDGHICK

04673

CITY: SEBAGO LAKE

DAY WHITMAN

SEALER CF WEIGHTS \& MEASURES

STANDISH SEBAGO

RFD BOX 1955 RTE 257

\section{CITY: SKOhHEGAN}

WALLACE, GARY

REA 3 ER OF WEIGHTS \& MEASURES SKOWHEGAN, ME 04976

CITY: SO PORTLANO

\section{SHERRARD KEITHR}

CITY OF SOUTH PHTS \& MEASURES

60 CHURCHILL PORTLAND

SO PORTLAND ME 04106

\section{CITY: SO WINDHAM}

BISHOP, JOHN R

SEALER OF WEIGHTS \& MEASURES

RED OF WINDHAM

RFD I FALMOUTH RD
SO WINDHAM. ME 04082

\section{CITY: SPRINGVARE}

LOWD GEORGE $F$

SEALER CF HEIGHTS \& MEASURES

SPRINGVALE, ME 04038
HERLIHY JAMES

WATERVILLE, ME $049 \mathrm{Cl}$

CITY: WEST GOULOSBORC

WESCOTT JR, CHARLES $M$

SEALER OF WEIGHTS E MEASURES

WEST GOULDSBORO. ME 04687

CITY: WILSON MILLS

WHITE: GLENWOOD

SEALER OF HEIGHTS \& MEASURES

CINCOLN PL

WILSON MILLS, ME 04293

CITV: WISCASSET

BLAGDON JR JOHN

SEALER OF HEIGHTS \& MEASURES

LANGDONET

WISCASSET. ME 04578

MAR YLAND

STATE

BARTON: JOHN W

WEIGHTS \& MEASURES SECTION

MARYLAND DEPT OF AGRICULTURE

50 HARRY S TRUMAN PARK WAY

$301841-5790$

BAUMANN. EUGENE E

WEIGHTS $E$ MEASURES SECTION MARYLAND DEPT OF AGR ICULTURE ANNAPOL IS MO 21401

BUTCHER, KENNETH S

WEIGHTS \& MEASURES

MD DEPT OF AGRICULTURE

5O HARRY S TRUMAN PARKWAY ANNAPOLIS: MD 21401

COT SORADIS CONSTANTINE Y WEIGHTS \& MEASURES SECTION MARYLAND DEPT OF AGR ICUE TURE A HARRY S TRUMAN PARKWAY $301841-5770$

DEGRANGE LACY H

WEIGHTS \& MEASURES SECTION 50 HARRY S TRUMAN PARKWAY

ANNAPOLIS: MD 21401
$301841-5790$

EAVES ROBERT D

WEIGHTS E MEASURES SECTION MARYLAND DEPT OF AGR ICULTURE 50 HARRY S TRUMAN PARKWAY ANNAPOLIS. MO 21401
$301841-5770$

EMGE DONNA

WEIGHTS 6 MEASURES SECTION MARYLAND DEPT OF AGR ICUE TURE 50 HARRY S TRUHAN PARKWAY ANNAPOLIS: MD 21401 
FRAILER - MICHAEL

WE I GHTS $\&$ MEASURES SECTION MAR YLAND DEPT OF AGRICULTURE 50 HARRY S TRUMAN PARKWAY ANNAPOLIS. MD 21401 $301841-5770$

\section{GAVER, TINA}

WEIGHTS \& MEASURES SECTION MAR YLAND DEPT OF AGRICULTURE 50 HARRY S TRUMAN PARKWAY ANNAPOLIS MO 21401

$301841-5770$

GORDON, DAN T

HEIGHTS \& MEA SURES SECTION MAR YLAND DEPT OF AGR ICULTURE 50 HARR Y S TRUMAN PARKWAY ANNAPOLIS MD 21401

$301841-5790$

\section{HALL. WILLIAM C}

WEIGHTS \& MEASURES SECTION MARYLAND DEPT OF AGRICULTURE 50 HARRY S TRUMAN PARKWAY ANNAPOL IS MD 21401 $301841-5770$

\section{HALPERN ETHAN}

WEIGHTS \& MEASURES SECTIDN MAR YLAND DEPT OF AGRICULTURE 50 HARR Y S TRUMAN PARKWAY ANNAPOL IS MD 21401 $301841-5770$

HAR SHMAN. RICHARD A WE IGHTS \& MEASURES SECTION MAR YLAND DEPT OF AGRI CULTURE 50 HARRY S TRUHAN PARKHAY ANNAPOLIS, MD 21401

$301841-5770$

HE IDER DAYID $W$

WE IGHTS \& MEASURES SECTION MAR YLAND DEPT OF AGRICULTURE 50 HARR Y S TRUMAN PARKWAY ANNAPOL IS, MO 21401 $301841-5370$

HOWARD, KENNETH W

WE IGHTS $\&$ MEASURES SECTION MAR YLAND DEPT OF AGRICULTURE 50 HARRY S TRUMAN PARKWAY ANNAPOL IS MD 21401

$301841-5770$

JONES, HARRY MAR YLAND DEPT OF AGRI CULTURE 50 HARRY $S$ TRUMAN PARKWAY ANNAPOLIS, MD 21401

$301841-5790$

\section{LAMBERT MARK}

WEIGHTS \& MEASURES SECTION MAR YLAND DEPT OF AGRI CUL TURE ANNAPOL IS MD 21401

$301841-5790$

LITTLEF IELD, LEONARD

WE IGHTS \& MEASURES SECTION MAR YLAND DEPT OF AGR ICULTURE 50 HARRY S TRUMAN PARKWAY ANNAPOL IS MD 21401

$301841-5790$
WEIGHTS \& MEASURES SECTION RYAND DEPT OF AGR ICUL TURE 50 HARRY S TRUMAN PARKWAY ANNAPOLIS. MD 21401

$301841-5770$

WILLERE BARBARA A MARYLAND DEPT OF AGR ICULTURE 50 HARRY $S$ TRUMAN PARK WAY ANNAPOLI S MD 21401 $301841-5770$

MUMMERT• DAVID

WEIGHTS \& MEASURES SECTICN MAR YLAND DEPT OF AGR ICULTURE 50 HARRY S TRUMAN PARKWAY ANNAPOLIS. MD 21401 $301841-5790$

PAYNE . EDWARD A

WEIGHTS \& MEASURES SECTION MARYLAND DEPT OF AGR ICULTURE 50 HARRY S TRUMAN PARKWAY ANNAPOLIS. MD 21401 $301841-5770$

PRICE. ARTHUR E

CHIEF MOOTOR FUEL INSPECTION COMPT OF TREAS GAS TAX DIV PO BOX 1751 TREASURY BLDG ANNAPOLIS MD 21404 301 269-3126

PRICE。 JAMES T

WEIGHIS E MEASURES SECTION MAR YLAND DEPT OF AGR ICULTURE 50 HARRY S TRUMAN PARKWAY ANNAPOLIS: MO 21401

$301841-5770$

RAMSBURG . KENNETH

WEIGHTS \& MEASURES SECTION MARYLAND DEPT OF AGR ICULTURE 50 HARRY S TRUMAN PARKHAY ANNAPOLIS MD 21401 $301841-5770$

RAWLS JOHN

WEIGHTS \& MEASURES SECTION MAR YLAND DEPT OF AGR ICUL TURE 50 HARRY S TRUMAN PARKWAY ANNAPOLIS, MO 21401

RHODES. THOMAS D

SUPERVISING INSPECTOR

MARYLAND DEPT OF AGR ICULTURE 12716 HOLIDAY LANE

BOWIE. MD 20716

$301841-5790$

RIOGELL RICHARD MARYLAND DEPT OF AGRICULTURE 50 HARRY S TRUMAN PARKWAY ANNAPOLIS MO 21401

$301841-5770$

SANTANA. EDWIN

WEIGHTS \& MEASURES SECTICN MARYLAND DEPT DF AGR ICUL TURE 50 HARRY S TRUMAN PARKWAY ANNAPOLIS MO 21401

SELLERS, AL

WEIGHTS \& MEASURES SECTION MAR YL AND DEPT OF AGR ICUL TURE 50 HARRY S TRUHAN PARK WAY ANNAPOLIS MD 21401

$301841-5790$ 
SHOCKLEY M RICHARD

MARYLAND DEPT OF AGRICULTURE 50 HARR Y TRUMAN PARKWAY ANNAPOLIS. MD 21401

$301841-5790$

STOCKMAN CHARLES $R$

WEIGHTS है MEASURES SECTION

DO HARRY S TRUMAN PKY

ANNAPOLIS MD 21401

$301454-5117$

STRAUB, LOUIS E

HEIGHTS AND MEASURES SECTION

50 HARRY S TRUMAN PARKWAY

ANNAPOLIS: MO 21401

$301841-5790$

SUMMERS TIMOTHY

METROLDGY TECHNICIAN

MAR YLAND DEPT OF AGRI CULTURE

50 HARRY S TRUMAN PARKWAY

ANNAPOL IS MD 21401

$301841-5790$

THOMPSON. RICHARD L

CHI EF OF WEIGHTS E MEASURES

MO DEPT OF AGRICULTURE ANNAPOLIS. MO 21401

$301841-5790$

TROUP.WILLIAM J

WE IGHTS \& MEASURES SECTION

MAR VLAND DEPT OF AGRICULTURE

50 HARRY S TRUMAN PARKHAY

ANNAPOL IS MD 21401

$301841-5770$

WILDER WHITIE

WE IGHTS \& MEASURES SECTION MARYLAND DEPT OF AGRICULTURE 50 HARRY S TRUMAN PARKWAY ANNAPOL IS: MO 21401

$301841-5790$

WOT THLIE RICHARD W

WE IGHTS \& MEASURES SECTION MARYLAND DEPT OF AGRICULTURE 50 HARRY $S$ TRUNAN PARKWAY ANNAPOLIS MD 21401

$301841-5770$
MBR

NCWM

MBR

NCWM

\section{MASSACHUSETTS}

\section{STATE}

BRADLEY JOHN

MASS DIVISION OF STANDARDS

ONE ASHBURTON PLACE

BOSTONL MA O2108
CARROLL CHARLES H

ASST DIRECTOR OF STANDARDS MASS DIVISION OF STANDARDS ONE ASHBURTON PLACE BOSTON MA 02108 $617727-3480$

CRITTENDEN SAMES

SUPER INTENDENT OF STANDARDS LB

MASS DIVISION OF STANDARDS

ONE ASHBÚRTON PLACE

BOSTON: MA 02108

$617727-5698$

GLENN JR. YIRGIL G

INSPECTOR OF STANDARDS

MASS DIVISION OF STANDARDS

ONE ASHBURTON PLACE

BOSTÔN. MA O2108

$617727-3480$

HANSBURY: THOMAS

INSPECTOR OF STANDARDS

MASS DIVISION OF STANDARDS

ONE ASHBUR TON PL

BOSTON. MA 02108

$617727-3480$

MASSARO, JOSEPH

INSPECTOR OF STANDARDS

MASS DIVISION OF STANDARDS

ONE ASHBURTON PLACE

BOSTON MA O2108

MCKEON MATTHEW C

INSPECTOR OF STANDDAROS

MASS DIVISION OF STANDAROS

ONE ASHBUR TON PLACE

BOSTON, MA O2108

$617727-3480$

MULLETT: JOHN

INSPECTOR OF STANDARDS

MASS DIVIS ION OF STANDARDS

ONE ASHBURTON PLACE

BOSTON. MA O2108

$617727-3480$

NIEMCZURA. DAVID

INSPECTOR OF STANDARDS

MASS DIVISION OF STANDARDS

ONE ASHBURTON PLACE

BOSTON: MA 02108

$617727-3480$

PACLAT HARVEY J

INSPECTOR OF STANDARDS

MASS DIVISION OF STANDARDS

ONE ASHBURTON PLACE

BOSTON: MA O21 C8

$617727-3480$

QUINLAN, FREDERICK F

INSPECTOR OF STANDARDS

MASS DIVISION OF STANDARDS

ONE ASHBUR TON PLACE

BOSTON. MA 02108

$617727-3480$

REAROON JR TI MOTHY J'

MASS DIVISION OF STANDAROS

ONE ASHBURTON PLACE

BOSTON. MA 02108

$617727-3480$

SCIBELLI I THOMAS

INSPECTOR OF STANOARDS

MASS DIVISION OF STANDARDS

ONE ASHBURTON PLACE

BOSTON. MA 02108

$617727-3480$ 
MITHE DCNALD W WNANDARDS

ASS DIVISION OF STANDARDS

NE ASHBURTON PLACE

OSTON MA 02108

, 7 727-3480

\section{ALSH JACK}

IVISION OF STANDARDS

INE ASHBUR TON PL

$317727-34802108$

IY: ACTON

FITZPATRICK MARK \& MEASURES

SEALER CF ACTON

4 FORREST RD

ACTON. MA OI720

$617897-9724$

TY: ACUSHNET

DAWSON CARLTON

CI TY SEALER

35 COULOMBE ST 2743

$617995-1141$

IITY: ADAMS

RUSEK, LEONARD

SEALER CF WEIGHTS $\&$ MEASURES

WEIGHTS 8 MEA SURES DEPT

TOWN HALL PARK ST

ADAMS MA OL 220

$413743-0795$

ITY: AG AWAM

DRAGHETTI - LOUIS D

INSPECTOR OF WTS \& MEAS

36 MAIN ST

AGAWAM MA 01001

$413786-0400 \times 232$

:ITY: AMESBURY

MORTON LELIS A

TOWN HALL ANNEX

$617388-2061$

CITY: AMHERST

STAIZT, HILLIAM

INSPECTOR OF W $E M$

TOWN OF AMHERST

AMHERST MA 01002

$413253-3453$

CITY: ANDOVER

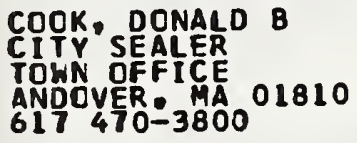

CITY: ARL INGTON

GALVIN. WALTER T

CITY SEALER

670 MASS AVE REAR

ARLINGTON: MA OIBIC

$617643-6700 \times 233^{\circ}$

CITY: ASHLAND

DRISCOLL GERALD J

CITY SEALER

Po $80 \times 342$

ASHLAND: MA 02178

MBR

NCWM

CITY: ATHOL

GREENE• J R

SEALER OF WEIGHTS AND MEASURES

584 MAIN STREET

ATHOL MA 01331

$617249-3062$

CITY: ATTLEBORO

BLISS: BRUCE J

NORTH ATTLEBORO MASS

156 RAYMOND HALL DR

ATTLEBORO: MA 027

CITY: AUBURN

DUHAMEL STEVEN

35 GOULDING OR

AUBBURN. MA OI5O1

617 832-3192

MBR

CITY: AVON

MCFARLANE LLOYD

CITY SEALER

62 GRANITE ST

AVON MA 02322

CITY: BARNSTABLE

GEILER - THOMAS F $\&$ MEASURES

MBR SEALER OF WFENSTABLE

367 MAIN ST

HYANNIS: MA 02601

CITY: BEDFORD

MCNEANY. THOMAS

SEALER OOF WEIGHTS - MEASURES

CITY OF BEDFORD

232 CARL ISLE RD

BEDFORD. MA 01730

$617275-8296$

CITY: BELCHERTOWN

MAGEE. DQUGLAS K

CITY OF BELCHERTOWN

BOWN HALLWERT MA 01007

413 323-5726 
QBR IEN口 RALPH M

SEALER OF WEIGHTS AND MEASURES

CITY PARK SEV

BEVERLY MA 01915

$617922-1316$

CITY: BI LLERICA

LAING, ALFRED D

SEALER CF WEIGHTS \& MEASURES

15 CCNCCRD RO 663-2611

CITY: BLACKSTONE

COLLIND FRANCIS J

CITY SEALER

MUNICIPAL CENTER 15 ST PAUL ST

BLACKSTCNE MA 01504

$617883-7656$

CITY: BOSTON

LYNCH\& JOHN

SEALER CFWEIGHTS \& MEASURES

BOSTON CITY HALL

ROOM 204

$60570 N^{2}$ MA 02201

MANNING PAUL H

WEIGHTS \& MEASURES

BOSTON: MA 02201

$617725-4540$

\section{CITY: BRIDGEWATER}

WOLFER HERBERT

SEALER OF WEIGHTS \& MEASURES

CITY OF BRIDGEHATER

510 NORTH ST

BR IOGEWATER

MA 02324

\section{CITY: BROCKTON}

\section{COYNE. MARK $P$}

CITY SEALER

CITY OF BROCKTON

CITY HALL RM BI2

BROCKTON MA 02401
$617580-1100$
$\times 158$

\section{CITY: BRCOKLINE}

HURLEY. WILLIAM F

BROCKLINE HEALTH DEPARTMENT

II PIERCE ST

BROOKLINE MA 02146

$617232-9020 \times 407$
LAING, ALFRED D

SEALER OF WEIGHTS \& MEASURES

ONE IRENE ST

BURLINGTON $272-0721$ MA 01803

CITY: CAMBR IDGE

LAFFIN. ROBERT K

CITY OF CAMBRIDGE

795 MASS AVE ROOM 211 CITY HAL

CAMBRIDGE MA

CITY: CANTON

SILVER • ROBERT E

CITY SEALER

TOWN HALL

CANTON: MA 02021

$617828-4734$

CITY: CHARLTON

KARNILA, VINCENT

CITY SEALER

HARRINGTON RD

CHARLTON MA O 1507

$617765-9066$

MBR
NCWM

CITY: CHATHAM

CONFALONE PASOUALE

SEALER WEIGHTS AND MEASURES

TOWN OF CHATHAM

549 MAIN STREET

CHATHAM. MA 02633

$617945-2100 \times 13$

CITY: CHELMSFDRD

FERREIRA. ANTHONY C

CITY SEALER

72 RIVERNECK RD

CHELMSFORD. MA 01824

$617452-8500$

CITY: CHELSEA

HANLON, JOHN J

SEALER OF WEIGHTS ANO MEASURES

CITY OF CHELSEA

5 OO BROAOWAY

MBR

CHELSEA. MA 02150

CITY: CHI COPEE

WILK. DOUGLAS J

CITY SEALER

413 598-8447 01013

CITY: CLINTON

DE CESARE RICHARD

SEALER OF HEIGHS AND MEASURES

TOWN OF CLINTON

CLINTON MA 01510

617 365-5511 
TY: COHASSET

BATES, LOT E TOWN OOF COHASSET MASS

365 BEECHWOOD ST

COHASSET. MA 02025

$617383-1129$

ITY: CONCORD

MAC WILLIAMS $C$ W WHO MEASURES

TOWN OF CONCORD

51 HILLSIDE AVE

CONCDRD. MA 01742

$617369-2398$

ITY: DALTON

BLAKESLEY, RALPH E SEALER CF HEIGH

462 MAIN ST

DALTON: MA 01226

$413684-0300$

\section{ITY: DANYERS}

CARR , JCHN

SEALER OF HEIGHTS \& MEASURES

TOWN OF DANVERS

IOWN HALL

DANVERS MA 01923

CITY: DARTMCUTH

AR SENAULT: THOMAS

CITY SEALER ST

15 PROSPECT ST 102748

$617993 \rightarrow 7398$

CITY: DEDHAM

DAMATAQ AL

CITY SEALER

TOWN HAL

DE DHAM: MA 02129

$617326-5770$

CITY: DENNIS

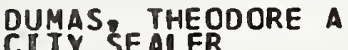

CITY SE ALER

BOX $D$

DENNIS, MA 02641

CITY: DIGHTON

HOFFSHIRE. PAUL SEALER CF WEIGHTS \& MEASURES 979 SOMERSET AVE $617669-6431$

\section{CITY: DRACUT}

BUR SEY RAYMOND

CITY OF DRACUT

TOWA HALL 62 ARLINGTCN ST

DRACUTT MA O 1826

MBR

DAWSON JR ALBERT

ITY SEALER

48 NEWBURY ST

DRACUT, MA O1826

$617453-8466$

CITY: DUDLEY

KILI AN, ALBERT

CITY SEALER

DUDLEY. MA 01570

617 943-3208

PERZICHINO. ANGELO

SEALER OF WEIGHTS 6 MEASURES

CITY DF DUDLEY

GLENDALE DR

DUDLEY: MA 01570

CITY: DUXBURY

BEERS DONALD

SEALER OF LEIGHTS $\&$ MEASURES

CITY OF DUXBURY

878 TREMCNT ST

DUXBURY: MA 02332

CITY: EAST BOSTON

DISTEFANO SR " CHARLES

CHIEF DEPUTY SEALER

WEIGHTS \& MEASURES DIVISION

135 SARATOGA ST

EAST BOSTON MA 02128

$617567-0307$

CITY: EAST BRIDGEWATER

CURLEY, JOHN F

SEALER

WEIGHTS AND MEASURES

175 CENTRAL ST

EAST BRIDGEWATER. MA 02333

$617378-7221$

CITY: EAST LONGMEADCW

BRA INERD, ROY E

SEALER OF WEIGHTS $\&$ MEASURES

TOWN OF EAST LONGMEADOW

128 LA SALLE ST

EAST LONGMEADOW. MA 01028

$413525-3198$

CITY: EASTHAMPTON

CURTIN. CHARLES H

CITY SEALER

8 SPRING ST

EASTHAMPTON. MA 01027
413 527-2196

CITY: EASTON

WOOOWORTH. BARRY L \& MEASURES

SEALER OF EASTON

TOWN OF EASTON 151 MASSAPOAG AVE

NORTH EASTON. MA 02352

$617238-1661$

CITY: EVERETT

ELL IDT T. LAWRENCE L

INSP WEIGHTS AND MEASURES

WEIGHTS \& MEASURES DEPARTMENT

CITY HALL ROOM 27

EVERETT: MA 02149

$617389-2100 \times 20$
MBR NCWM 
PRZYBYSZEWSKI FRANK E

SEALER CF HEIGHTS \& MEASURES TOWN OF FAIRHAVEN

40 CENTRE ST

FAIRHAVEN MA 02719

$617992-5416$

CITY: FALMOUTH

CROSSEN J JAMES M S \& MEASURES TOWN OF FALMOUTH

TOWN HALL

FALMOUTH MA 02540

617 548-7611

CROSSEN, JAMES M

DEPTER WEIGHTS-MEASURES

TOWN HALL SQUARE

FALMOUTH. MA 02541

\section{CITY: FITCHBURG}

PIERCE ROBERT J

INSPECTOR

WEIGHTS \& MEASURES DEPT

CITY HALL 718 MAIN ST

FITCHBURG. MA 01420

617 343-7012

CITY: FOXBORO

MCKAY, RAYMOND E SEALER CF WEIGHTS AND

40 SOUTH ST

FOXBORO: MA 02035

$617543-5301$

\section{CITY: FR AMINGHAM}

BARTOLINI MARIO G

DEPUTY INSPECTOR W \& M

TOWN OF FRAMINGHAM

MEMCRIAL BUILDING

FRAMINGHAM MA 01701

$617620-4838$

CITY: FRANKLIN

MERCLLA BERNARO

SEALER CF WEIGHTS $E$ MEASURES

INSP OFF/150 EMMONS ST

FRARKLIN. MA 02038

$617528-1749$

CITY: FREETOWN

SARCSIEK, JULIUS J

TOWN OF FREETOWN

3 NCRTH MAIN ST

ASSONET. MA 02702

$617763-4017$

CITY: GARDNER

HIRONS. FRANK K

SEALER OF WEIGHTS \& MEASURES

CITY OF GARDNER

GIIY HALL RM 17

$$
\text { 632-1212 }
$$

MBR

NCWM

MBR

NCWM

MBR

MBR

NCWH
CITY: GROVELAND

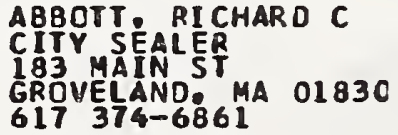

CITY: HAMILTON

OIXON GEORGE

SEALER OF WEIGHTS 8 MEASURES

CITY OF HAMILTON

50 SHARON RD

HAMILTON. MA 01982

468-1359

A 01230

MEASURES

536

CITY SEALER

GEDENTRAL ST 01830

617 352-2914

MEASURES

FT ACRES

\section{PT} ND MEAS DEP 4 DED

CITY: GRANBY

\section{CITY: GREAT BARRINGTON}

\section{CITY: GREENFIELD}

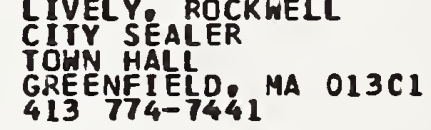

\section{CITY: GROTON}

S 6 MEASURES

SEALER 


\section{CTY: HANOVER}

HEST. ARTHUR C

CITY SEALER

3)

$617826-2261$

ITY: HANSON

SERRILLA FRANCIS $X$

CITY SEALER

26 SLEI GH DRIVE

HANSON MA 02341

$617826-6588$

ITY: HARWICH

DOUBLEDAY WILLIAM A

TOWN OF HARWICH

23 JUL IA COURT

HARHICH MA 02645

$617432-6741$

ITY: HAYERHILL

HALLL RALPH

CIIY OF HAVERHILL

CIIY OF HALR

HAVERHILL NA 01830

$683-9813$

:ITY: HINGHAM

WALLACE D THOMAS JN'D MEASURES SEALER WEIGHTS 7 ST

HINGHAM.MA 02043

\section{CITY: HOLBROOK}

HAITE

CITY SEALER

TOWN HALL

HOLBROOK. MA 02343

$617767-4312$

CITY: HOLDEN

COOK KENNETH

CITY SEALER

IAUREI HOOD RD

RUTLAND. MA O1543

617 829-4444

\section{CITY: HOLLISTON}

BRENNEN R ROBERT S SEALER CF WEIGHTS PI 0 BOX E

WESTBORO 617 MA 01581

$\varepsilon$ MEASURES

CITY: HOLYOKE

FORTIN EARL A

CIIY SEALER

MBR

CITY HALL

HOLYOKE HA 01040

413 534-2169

MBR

NCWM

\section{CITY: HOPKINTON}

LEAL JR, GEORGE F

SEALER OF WEIGHTS \& MEASURES

CIIY OF HOPKINTON

238 WEST MAIN ST

HOPKINTON MA O1581

$617435-4286$

CITY: HOUSATONIC

ABEROALE JOSEPH

CIIY SEALER

LINDA LANE, MA 01236

413 274-3813

CITY: HULL

COHEN, ELLIOTT

SEALER OF WEIGHTS 6 MEASURES CITY OF AULL 8 O ST HULL: MAA 02045

CITY: IPSWICH

BEDARD. NORMAN J

SEALER HEIGHTS \& MEASURES

TOWN OF IP SHICH MASSACHUSETTS

CIO BOARD OF HEALTH

IPSWICH. MA OI 938

$617744-0254$

CITY: KINGSTON

CAVICCHI MICHAEL F

CITY SEALER

KINGSION FIRE DEPT/MAPLE ST

KINGSTON, MA 02360

617 585-2521

CITY: LAKEVILLE

STAPLES PERCIVAL AND MEASURES

DIVISION OF STANDARDS

LAKEVILLE. MA 02346

$617822-3452$

CITY: LANCASTER

POIRIER: LAWRENCE J MEASURES

CITY OF LANCASTER

LE6 ELM ST MA 01523

$617534-8112$

CITY: LAWKENCE

BERARD STEPHEN R

CITY SEALER

LAWRENCE MA 01843

$617685-4296$

CITY: LEE

DARBE CHARLES E

CITY SEALER

LEE MA O1 238 
DAIGLE ROBERT $B$

CITY SEALER

LEICESTER MA 01524

CITY: LENOX

TANE. ROSS B

CITY SEALER

LENCHURCH ST 240

$413637-0908$

CITY: LEOMINSTER

POIRIER LAWRENCE J

62 MECHANIC ST

LEOMINSTER MA 01453

CITY: LEXINGTON

HAM JR, RICHARD W

SEALER OF WEIGHTS AND MEASURES MBR TOHN OF LEXINGTON

1625 MASS AVE

LEXINGICN MAA 02173

CITY: LINCOLN

JOHNSON ERNEST LL \& MEASURES

TOWN OF LINCOLN

IOWNOFFICE 82 LO

617 259-8 850

\section{CITY: LITTLETON}

BERNIER ROLAND

CITY SERLER

LITTLETCN MA 01460

8ERNIER \& ROLAND

SEALER OF WEIGHTS \& MEASURES

20 FOSTER ST

IITTLETCN MA 01460

CITY: LONGMEADOW

CLARK, RCBERT E

SEALER OF WEIGHTS E MEASURES JOWN OF LONGMEADOW

20 ILLIAMS ST

413 L67-5433 MA 01106

CITY: LOWELL

ROUSSEAU PAUL

CITY OF LOWELL

CITY HALL HERRIMACK ST

$617454-8821 \times 224$

HILK DOUGLAS

CITY SEALER

488 CHAPIN ST 2020

$413598-8447$

CITY: LUNENBURG

DAVIES. LAUREN W

CITY SEALER

830 MASS AVE

LUNENBURG

CITY: LYNN

MILMORE L EO

CITY SEALER

CITY HALL

LYNN. MA 01803

$617598-4000 \times 176$

CITY: LYNNFIELO

MICHALSKI - EDWARD J

SEALER OF WEIGHYS ANO MEASURES

120 OERBY RD

$617284-46442151$

CITY: MALDEN

MBR

NCHM

MBR

NCHM

KING JOHN J

SEALER OF WEIGHTS AND MEASURES

CIY DF MALDEN

356 COMMERCIAL ST

$617324-6600 \times 175$

CITY: MANCHESTER

SWEM. H RALPH

ITY SEALER

98 PLEASANT ST

MANCHESTER MA 01944

617 526-1808

CITY: MANSFIELD

TERRENZ

CITY SEALER

81 CHILSON AVE

MANSFIELD MA 02048

CITY: MARBLEHEAD

ITUS CALYIN E

ÁBBOT SEALER

MARBLEHEAD. MA 01945

$617339-8574$

CITY: MARL8ORO

BOUVIER JR - EDWARD J SEALER OF HEIGHTS AND MEASURES NE

367 WEST HILL RD

MARLBORO, MA 01752

$617481-0481$ 


\section{IITV: MARSHFIELD}

LAFOREST LEONARD

CITY SEALER

TOWN HAOL

${ }_{617}^{M A R S H F I E L D} 3_{3}^{\text {MA }} 02050$

ITY: MATTAPOISETT

SEARS. ALFRED T

CITY SEALER

MAITAPOISEIT. MA 02739

$617758-3758$

ITY: MEDFIELD

\section{RIOUX PATRICIA}

27 GREEN ST

MEDFIEL, 3 MA 02052

:ITY: MEOFORD

VINCI: WILLIAMS

CIYY OF MEDFORD CIIY HALL

MEDFORD. MA 02155 $396-5500 \times 463$

\section{CITY: MEOWAY}

PARCHESKY PHILIP

SEALER WEIGHTS \& MEASURES

155 VILLAGE ST

MEDWAY MA 02053

$617533-8190$

CITY: MELROSE

DWYER, MICHAEL

DWYER MICHAEL

562 MAIN ST

MELROSE MA 02176

$617665-2399$

\section{CITY: METHUEN}

HALL, RALPH H

CITY SEALER

14 KENDALL ST

METHUEN. MA 01844

$617683-9813$

\section{CITY: HIDDLEBORO}

NORVISH CHARLES S

CITY SE ALER

MIODLEBORO. MA 02346

CITY: MILFORD

PANCRESE, ERNEST M

CITY SEALER

3 MANELLA AVE

MILFORD MA 01757

$617473-3577$
CITY: MILLBURY

BRADY, FRANCIS J

HEIGHTS $\&$ MEASURES

129 H HAIN ST

MILLBURY MA 01527

$617865-2632$

CITY: MILLIS

HOWIE. PAUL

SEALER HEIGHTS \& MEASURES

RAILROAD AVE

MILLIS. MA 02054

$617376-8631$

CITY: MILTON

WILLIAMS PAUL D

CITY SEALER

525 CANTDN AVE

MILTON MA O21 86

CITY: MONSON

MBR

SERRATO ALBERT J

5 IT MAIN ST

51 MAIN ST 01057

$413267-3719$

CITV: MONTAGUE

NEWTON ROGER

CITY SEALER

TAYLOR HILL ROAD

MONTAGUE MA OI351

$413367-2237$

\section{CITY: NANTUCKET}

NCWM

RAY RICHARD L

SEALER OF WEIGHTS AND MEASURES TOWN OF NANTUCKET

JOWN ANO COUNTY BLDG

NANTUCKET MA 02554

$617228-9481$

\section{CITY: NATICK}

MULVEY - JOSEPH J J WND MEASURES TOWN OF NATICK

13 EAST CENTRAL ST

NATICK. MA 01760

$617651-7230$

\section{CITY: NEEDHAM}

GILL. JOHN J

SEALER OF WEIGHTS AND MEASURES

TOWN OF NE EDHAM

36 HOMESTEAD PARK

NEEDHAM. MA 02194

$617444-6368$

CITY: NEW BEDFORD

PRZYBYSZEWSKI FRANK E

SEALER OF WEIGHTS \& MEASURES

CITY OF NEW BEDFORD

306 LIBERTY ST

NEW BEDFORD. MA 02740

MBR

NCWM 
CITY: NEWBURYPORT

MUR PHY AL

SEALER WE IGHTS AND MEASURES

CIIY OF NEWBURYPORT

CETY HALE PLEASANT ST

$617465-3444$

CITY: NELTON

SILVER ROBERT E

CITY SEALER

100 COMM AVE/NEWTON CITY HALL

NEHTON MA 02159

CITY: NORFOLK

HILOEBRANDT. PETER S

CITY SEALER

LELAND IRDAD

NORFOLK. MA 02056

$617528-2613$

CITY: NORTH ADAMS

BIRKLANE, BERNIE

INSPECTOR-SEALER OF WTS \& MEAS

CITY DF NORTH ADAMS

I0 MAIN ST

NORTH ADAMS: MA 01247

$413663-6495$

CITV: NORTH ANDOVER

ROBERTS E E

15 BRIGHTWOOD AVE

NORTH ANDOVER, MA O1845

$617 \quad 682-6752$

CITY: NORTH ATTLEBORC

BL. ISS BRUCE

CITV SEALER

156 RAYMCND HALL DR

NORTH ATTLEBORO. MA 02760

CITY: NORTH READING

MORROW ROBERT W

CITY SEALER

24 LECLAIR ST

NORTH READING. MA 01867

$617664-5879$

\section{CITV: NORTHAMPTON}

FOURNIER, CRAIG

INSPECTCR OF WEIGHTS $\&$ MEAS

CIVY OF NORTHAMPTDN

MUNICIPAL BLOG

NOR THAMPTON: MA 01060

CITV: NORTHBORO

BUTT ARTHUR E

SEALER OF WEIGHTS E MEASURES

63 MAIN ST THBDROUGH

NORTHBORO MA 01532
$617393-6761 \times 213$
CITV: NORTHBRIOGE

GOSHGAR I AN: MICHAEL

CITY SEALER

CITY OF NORTHBRIDGE

315 LINWOOD AVE

WHITINSVILLEO MA 01588

$617234-8547$

CITY: NORTON

CAMARA。 AMERICO F

CITY SEALER

123 FREEMAN ST

NORTONO MA O2766

CITY: NORWELL

GARDNER。 ALTON C

CITY SEALER

27 STETSON ST

NORHELL: MA 02061

CITY: NORWOOD

BROWN, FREDERICK

SEALER OF HEIGHTS \& MEASURES

TOWN OF NORWODO MASS

32 GREENWICH RD

NOR WOODD. MA 02062

$617762-7197$

CITY: ORANGE

GREENE尺 J R

584 MAIN ST

ATHOL MA 01331

$617 \quad 249-3374$

CITY: OXFORD

SMOLENSKI HENRY E

CITY SEALER

CHARLTON ST

OXFORD. MA 01540

617 987-2353

CITY: PALMER

CLARK ROBERT E

CITY SEALER

103 RHINEBECK AVE

SPRINGFIELD. MA OLI 129

CITY: PEABODY

SHAHNAIAN. GEORGE

INSPECTOR OF WEIGHTS \& MEASURE

CITY HALL LOWELL ST

PEABOOY MA O1 960

\section{CITV: PEMBRCKE}

KELLY JR, RQBERT $M$

SEALER OF WEIGHTS $\&$ MEASURES

CITY OF PEMBROKE

5 SPR ING ST

PEMBROKE MA 02359

617826-6081

CITY: PEPPERELL

SCHULTZ RUDOLLPH F

212 SDUTH RD

01463 
MATHEWS JAMES E TOWN OF SHARON MA 2 MCRSE ST

SHARON MA 02067

CITY: SHIRLEY

MCCARTHY. HILLIAM F

CITY SEALER

CENTER TOWN HALL

SHIRLEY MA 01464

$617425-4041$

CITY: SHREWSBURY

KNIPE JR, JOHN F

CITY SEALER

1OO MAPLE AVE
SHREHSBURY MA 01545
$617942-6666$

CITY: SOMERSET

CAMPBELLO WILLIAM \& MEASURES SEALER H OF WWE RD SOMERSET. MA 02725

$61767.6-1004$

CITY: SOMERVILLE

MALLARD, EDWIN

CITY SEALER

WEI GHTS E MEA SURES DEPT

$P W D$ BUILDING FRANEY RD

SOMERYLLE MA 02143

$617625-6600 \times 166$

CITY: SOUTH HADLEY

DALY DAVID

SEALEROFWEIGHTS AND MEASURES TOWN OF SOUTH HADLEY

94 IYMAN ST

SOUTH HADLEY. MA 01075

CITY: SOUTHBORO

PHANEUF JR. EDGAR A

SEALER OF HTS AND MEASURES

TOWN OF SOUTHBORO

17 COMMON ST (TOWN HOUSE)

SOUTHBORO. MA 01772

$617485-0710 \times 26$

CITY: SOUTHBRIDGE

VARIN, ROLAND A

SEALER CF WEIGHTS \& MEASURES

CITY DF SOUTHBR IDGE

SOUTHBRIDGE MA 01550

CITY: SOUTHWICK

SP ILLANE, ALBERT

CITY SEALER

152 FEEDING HILLS RD

413 SOU I CK 30

\begin{tabular}{|c|c|}
\hline & CITY: SPENCER \\
\hline & $\begin{array}{l}\text { LAMBERT. RAYMOND A } \\
\text { SEALER . TOWN HALL } \\
\text { SPENCER TOWN } 01562 \\
\text { SPENCER: } \\
617885-3172\end{array}$ \\
\hline & CITY: STONEHAM \\
\hline & $\begin{array}{l}\text { RICH GEORGE } \\
\text { CITY SEALER } \\
20 \text { DUNCKLEE AVE } \\
\text { STONEHAM. MA } 02180 \\
617438-2584\end{array}$ \\
\hline & CITY: STOUGHTON \\
\hline $\begin{array}{l}\text { MBR } \\
\text { NCWM }\end{array}$ & $\begin{array}{l}\text { NOE VINCENT } \\
\text { CITY SEALER } \\
20 \text { BROOKDLE RD } \\
\text { STOUGHTON. MA O2072 } \\
617344-2341\end{array}$ \\
\hline & CITY: STURBRIDGE \\
\hline & $\begin{array}{l}\text { PROVOST ROBERT } \\
\text { CITY SEALER } \\
\text { TOWN HALL BOX } 645 \\
\text { STURBRIDGE MA } 01566 \\
617347-3639\end{array}$ \\
\hline & CITY: SWAMP SCOTT \\
\hline & $\begin{array}{l}\text { OHARE JOHN F } \\
\text { CITY SEALER } \\
37 \text { REDINGTON ST } \\
\text { SWAMPSCOTT MA } 01907 \\
617593-5476\end{array}$ \\
\hline & CITY: SWANSON \\
\hline & $\begin{array}{l}\text { BERNIER JR LEO L A } \\
\text { SEALER WEIGHTS AND MEASURES } \\
\text { I56 HILDER ST } \\
\text { SWANSEA. MA } 02777\end{array}$ \\
\hline & CITY: TAUNTON \\
\hline & $\begin{array}{l}\text { DOROTO. ARTHUR } \\
\text { SEALER } \\
\text { CITY OF TAUNTON } \\
330 \text { EAST BRITANNIA STREET } \\
\text { TAUNTON. MA O2780 }\end{array}$ \\
\hline & CITY: TEMPLETON \\
\hline & $\begin{array}{l}\text { WHALENE JAMES M } \\
\text { CITYSEALER } \\
23 \text { MEMORIAL ST } \\
\text { BALDWINVILLE. MA } 01436 \\
617939-5.505\end{array}$ \\
\hline & CITY: TEHKSBURY \\
\hline & $\begin{array}{l}\text { SULLIVAN, FRANK J } \\
\text { CITY SEALER } \\
4 \text { MARION DR } \\
\text { TEHKSBURY MA } 01876 \\
617851-2055\end{array}$ \\
\hline & CITY: TOPSFIELD \\
\hline & $\begin{array}{l}\text { CARYER JOSEPH H } \\
\text { SEALER OF WEIGHTS \& MEASURES } \\
\text { TOP SFIELD } \\
18 \text { GAEL ST } \\
\text { TOP SFIELD MA } 01983 \\
617887-5758\end{array}$ \\
\hline
\end{tabular}


I: TOUNSEND

FYNOLDS Y LYLEE AND MEASURES

jWN OF TOWNSEND

i) HIGHLAND S

]WNSEND MA 01469

(1) 597-2245

1: UXBRIDGE

ICHALIK, PETER

ITY SEALER

G GRAN ITE ST

XBRIDGE MA 01569

$17278-2146$

IY: WAKEFIELD

ARISELLA. PASQUALE J́̉ MEASURES

OWN OF WAKEFIELD

15 NAHANT ST

AKEFIELD, MA 01880

$.17245-3741$

IY: WALPOLE

:OREY ALFRED $B$

NSPECTOR

IE IGHTS \& MEASURES

- JCAL AVE

TALPOLE: MA 02081
SI $7668-0030$

IY: WALTHAM

CONNOR: RUSSELL M

CITY OF WAL THAM

$14 \mathrm{CHURCH}$ ST

WAL THAM. MA 02154
$617893-4040 \times 275$

TY: WARE

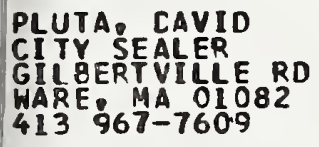

PLUTA CAVID

CI TY SEALER RD

WARE MA OIO82

$413967-7609$

ITY: HAREHAM

CORWIN, DONALD A

SEALER

TOWN HALL

HAREHAM MA 0257

MBR

$617295-0800 \times 230$

CORWIN DONALD A

CIJY SEALER

WAREHAM MA 02571

617 295-0800

\section{ITY: WATERTOWN}

MAHONEY JOSEPH

CITY SEALER

149 MAIN ST

WATERTOWN MA 02172

$617924-0460$

\section{CITY: WAYLAND}

ATKINSON , COUR TNEY

CITY SEALER

120 SYCAMORE ST

BELMONT: MA 02178

CITY: WEBSTER

GODZIK. FRANK

CITY SEALER

58 WHITCOMB ST

WEBSTER MA O5170

CITY: WELLESLEY

LAAK FRANK

SEALER OF WEIGHTS

TOWN OF HELLESLEY

525 WASHINGTON ST

$617235-0262$

CITY: WEST BOVLSTON

DABY J JOHN N

CITY SEALER

44 WORCESTER ST

WEST BOYLSTON. MA 01583

$617835-3733$

CITY: WEST BRIDGEWATER

TURNER. WARREN A

CITY SEALER

24 SCOTLAND ST

WEST BRIDGEWATER, MA 02379

617 586-5681

CITY: WEST SPRINGFIELD

YOUNGS RICHARD

INSPECTOR OF WEIGTS \& MEASURES

TOWN HALL
WEST SPRINGFIELDE MA 01089
$413781-7550 \times 237$

MBR

NCWM

CITY: HESTBORO

BRENNEN, R S

SEALER OFF HEIGHTS AND MEASURES

WESTBORO MA

HESTBORO. MA 01581

617 366-8121

CITY: WESTFIELD

PARENZO, DANA $\int$

SEALER ÖF WEIGHTS \& MEASURES

MBR

MUNICIPA BIDG/59 CCURT ST

WESTFIELD MA 01085
$413568-9181 \times 241$

CITY: WESTFORD

WELLS, HUNTINGTON \& MEASURES

CIIY OF WESTFORD

123 CONCCRD RD

WESTFORD MA 01886 692-8066 
ATKINSON COURTNEY A

SEALER OF WEIGHTS \& MEASURES

CITY OF HESTON

TOWN HALL BOX 378

WESTON, MA OLZ193

$893-7320$

CITY: WESTPORT

COOKSON S KENNETH SEALER OF HEIGHS \& MEASURES

TOWN OF WESTPORT MA

42 EAST BR IGGS RD

HE STPORT HA 02790

CITY: WESTHOOD

MOYNIHAN VINCENT P

SEALER CF WEIGHTS \& MEASURES

CITY OF WESTHWOD

WB WESTFIELD ST 090 $326-6331$

CITY: WEYMOUTH

O KEEFE PAUL

CITY SEALER

32 VICTORIA AVE

BRAINTREE MA 02188

$617848-4888$

CITY: WHITMAN

GODBOUT ROBERT

SEALER OF HEIGHTS \& MEASURES

CITY OF WHITMAN

495 FRANKLIN ST

$447-3090$

CITY: WILBRAHAM

MALZENSKI EOWARD

SEALER OF WEIGHTS

II SUNNYSIDE TERR WILBRAHAM: MA 01095
$596-3250$

6 MEASuRES

\section{CITY: WILLIAMSTOWN}

GEORGE。 FREDERICK

SEALER OF WEIGHTS MEASURES

196 BRIDGES RD

$413458-5205$. MA 02167

\section{CITY: WILMINGTON}

FARRELL MARTIN P

SEALER OF HEIGHTS \& MEASURES

CITY OF WILMINGTON

HI MARCUS RD

$$
\text { 65 T-7471 } 01887
$$

CITY: HINCHENDON

KACHINSKY PAUL R

SEALER CF WEIGHTS \& MEASURES

20 BALDWINVILLE STATE RD

HINCHENDON MA STA

$$
\text { 297-0218 }
$$

\section{CITY: HINCHESTER}

CAPONE JOSEPH

SEALER OF WEIGHTS $\&$ MEASURES CITY OF HINCHESTER

TOWN HALL

HINCHESTER MA 02890

CITY: WINTHROP

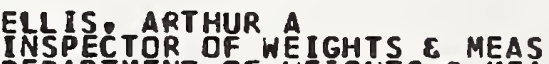
DEPARTMENT OF WEIGHTS $E$ MEAS 95 CIRCUIT RD HINTHROP MA 02152

$617846-3720$

CITY: WOBURN

MBR

GARYEY. RALPH

SEALER OF HEIGHTS \& MEASURES CITY OF HOBURN CITY HALL HOBURN. MA 01801

CITY: HORCESTER

MADAIO. PHILLIP

SEALER AND INSPECTOR

HEIGHTS AND MEASURES DEPT 37 LEE ST WORCESTER, MA 01606 $617853-8754$

\section{CITY: HRENTHAM}

HEINZ SR, ROBERT H

SEALER OF WEIGHTS $\&$ MEASURES CITY OF WRENTHAM

1092 HEST ST WRENTHAM MA 02093

CITY: YARMOUTH

MCBR I DE JAMES

1 CANVASBACK LANE

YARMOUTH: MA 02673

$617394-2430$

\section{MICHIGAN}

STATE

ABBOTT BERNARD

INSPECTOR DEPT OF AGRICULTURE $P$ BOX 30017

LANSING. MI 48909

517 373-1060

ARQUETTE MICHAEL

MICHIGAN DEPT OF AGRICULTURE $P$ O $80 X 30017$

LANSING. MI 48909

$517373-1060$

AUYER DIANE

INSPECTOR

MICHIGAN DEPT OF AGRICULTURE PO BOX 30017 
ALAZE RON

ICHIGAN DEPT OF AGRICULTURE C BOX 30017

ANSING, MI 48909

ARRON FEL IX

NSPECTOR

ICHIGAN DEPT OF AGRICULTURE

0 BoX 30017

ANSING MI 48909

\section{IELLOWS \& PATRICIA}

IICHIGAN DEPT OF AGRICULTURE

, O BOX 30017

ANSING MI 373060909

BENNETT CELESTE

IN SPECTCR DEPT OF AGRICULTURE $P$ BOX 30017

LANSING MI 48909

$517373-1060$

BIRGY HAROLO

METRCLIGAN DEPT OF AGRICULTURE P C BOX 30017

LANSING. MI 48909

$517373-1060$

BLEILER EUGENE

INSPECTR

MICHIGAN DEPT OF AGRICULTURE $P$ O BOX 30017

LANSING MI 48909

BRACE SUSAN

INSPECTOR DEPT OF AGRICULTURE $P$ O BOX 30017

LANSING. MI 48909

$517373-1060$

\section{BRACFORD, NANCY}

MICHIGAN DEPT OF AGRICULTURE PCBOX 30017

LANSING MI 48909

$517373-1060$

BRATUS, TOM

INSPECFOR

MICHIGAN DEPT OF AGRICULTURE

$P$ O BOX 30017

LANSING. MI 48909

$517373-1060$

BRE TISCHNEIDER, HENRY

INSPECTCR

MICHIGAN DEPT OF AGRICULTURE

$P$ BOX 30017

LANSING $373-106048909$

BURROWS D JAMES H HURES INSPECTOR

MICHIGAN STATE

1009 GRANT STREET

NILES MI 49120

$616925-2461$

BUTLER, KAREN

INSPECTCR

MICHIGAN DEPT OF AGRICULTURE

$P$ BOX 30017

LANSING. MI 48909

$517373-1060$
COOPER: ARTHUR

INSPECTOR

MICHIGAN DEPT OF AGRICULTURE $P$ BOX 30017

LANSING. MI 48909

517 373-1060

DIANICH. CHARLENE

INSPECTOR

MICHIGAN DEPT OF AGRICULTURE

$P$ D BOX 30017

LANSING. MI 48909

517 373-1060

DURN ING, GWEN

INSPECTOR DEPT OF AGRICULTURE $P$ O BOX 30017

LANSING. MI 48909

$517373 \div 1060$

ELL IOTT MAX

MICHIGAN DEPT OF AGRICULTURE $P$ BOX 30017

LANSING. MI 48909

$517373-1060$

ERICKSON, BILL

INSPECTOR BILL OF AGRICULTURE $P$ BOX 30017

LANSING. MI 48909

517 373-1060

NCHM

FEHINS CLARE

INSPEC TOR REPT OF AGRICULTURE P $\mathrm{BOX} 30017$

LANSING. MI 48909

517 373-1060

FORTIN NEAL

INSPECTOR MICHIGAN DEPT

5 ANSING. MI 48909

GABRIEL PHILIP

INSPECTOR DEPT OF AGRICULTURE $P$ B BOX 30017

517 IT3- 1060

GARFIELD, MIKE

INSPECTOR DEPT OF AGRICULTURE P D BOX 30017

LANSING. MI 48909

$517373-1060$

GAWEL TERRY

INSPECTOR

INICHIGAN DEPT OF AGRICULTURE $P$ O BOX 30017

LANSING $373-106048909$

GIGER SYLVIA

MICHIGAN DEPT OF AGRICULTURE $P$ O BOX 30017

LANSING. MI 48909

$517373-1060$

GIVENS WENDY

INSPECTOR DEPT OF AGRICULTURE PO BOX 30017

LANSING. MI 48909

517 373-1060 
GQLDIN LARRY

MICHIGAN DEPT OF AGRICULTURE $P$ O BOX 30017

LANSING: MI 88909

GRABAN T $C$

MICHIGAN DEPT OF AGRICULTURE $P$ BOX 30017

$517373-10608909$

HAFNER, AL

FOOD TECHNOLOGIST

MICHIGAN DEPT OF AGRICULTURE $P$ O BOX 30017

LANSING. MI 48909

517 373-1060

HARRINGTON, JIM

INSPECTOR

MICHIGAN DEPT OF AGRICULTURE $P$ BOX 30017

LANSING.MI 48909

HEFFRON, EOHARD C

CHIEF FOODO DIVISION

OT LANSING. MI 48909

$517373-1060$

HEI CEN JOHN

MICHIGAN DEPT OF AGRICULTURE $P$ C $80 X 30017$

LANSING. MI 48909

HOI TENG A LAURA

INSPECTOR

MICHIGAN DEPT OF AGRICULTURE

PO BOX 30017

$517373-106048909$

HOPP HERBERT

INSPECTOR

MICHIGAN DEPT OF AGRICULTURE $P 0$ BOX 30017

LANSING $373-106098909$

HOWELL BNETH

MICHIGAN DEPT OF AGRICULTURE PO BOX 30017

LANSING MI 48909

IACOPELLI. FRANK

MICHIGAN DEPT OF AGRICULTURE PO BOX 30017

LANSING $\frac{M 1}{373}-10608909$

JASCN, ROBERT

INSPECTCR

MICHIGAN DEPT OF AGRICULTURE

$P 0$ BOX 30017

517 STH-1060 48909

JESSUP MALCCLM

MICHIGAN DEPT OF AGRICULTURE

P BOX 30017

517 L
JONES \& KEN

INSPECTOR

MICHIGAN DEPT OF AGRICULTURE $P$ BOX 30017

LANSING. MI 48909

JORDAN RODNEY

AICHIGAN DEPT OF AGRICULTURE

$P$ BOX 30017

$517373-106048909$

JUHASZ MICHAEL

INSPECTOR

MICHIGAN OEPT OF AGRICULTURE PO $80 \times 30017$

$517373-106048909$

KAZANOWSKI ANN

FOOD SPECI ALIST

FOOD \& HEIGHTS 6 MEASURES DIV $4 T H$ FLOOR OTTAWA TOHER N

LANSING. MI 48909

MBR

NCWM 313 693-2265

KOZLOWSKI. JANE

MICHIGAN DEPJ OF AGRICULTURE

$P 0$ BOX 30017

LANSING. MI 48909

$517373-1060$

LALO JACK

NSPECTOR

HICHIGAN DEPT OF AGRICULTURE P BOX 30017

517 S N N

LOUNSBURY. TOM

MICHIGAN DEPT OF AGRICULTURE P O 80X 30017

LANSING. MI 48909

517 373-1060

LUDLOW JACK

MICHIGAN DEPT OF AGRICULTURE

$P$ BOX 30017

517 ING 1060

MERCER PAT

MICHIGAN DEPT OF AGRICULTURE $P$ D BOX 30017

LANSING $373-10608909$

MILLER, MARV

INSPECTOR MICHIGAN DEPT OF AGRICULTURE

LANSING. MI 48909

MORGAN JAY

MICHIGAN DEPT OF AGRICULTURE

$P$ O BOX 30017

LANSING. MI 48909

517 373-1060

NAGELE, FRANK

WEIGHTS E MEASURES SPECIAL IST MI DEPT OF AGR ICUL TURE

PO BOX 30017

LANSING MI $373-106048909$ 
IAVARRETTE, VIC

NSPECTOR

IICHIGAN DEPT OF AGRICULTURE

BOX 30017

ANSING MIJ-1060 48909

JEAL F FRER IC

INSPECTCR

IICHIGAN DEPT OF AGRICULTURE

O BOX 30017

ANSING MI 48909

$517373-1060$

PALADI F ED

INICHIGAN DEPT OF AGRICULTURE $P$ B BOX 30017

LANSING MI 48909

PATTERSON, COLLEEN

MICHIGAN DEPT OF AGRICULTURE P BOX 30017

LANSING. MI 48909

$517373-1060$

PAUL: MARGARET

INSPECTCR

MICHIGAN DEPT OF AGRICULTURE

$P$ O BOX 30017

LANSING. MI 48909

$517373-1060$

\section{PAVLAT, ALLAN}

INSPECTCR

MICHIGAN DEPT OF AGRICULTURE

$P$ O BOX 30017

LANSING. MI 48909

$517373-1060$

PI TYNSKI EDNA

MICHIGAN DEPT OF AGRICULTURE

P C BOX 30017

LANSING

REEDY RONALD

INSPECTOR

MICHIGAN DEPT OF AGRICULTURE

PO BOX 30017

ANSING MI 48909

$517373-1060$

RENDEL, BERNARD

INSPECTCR

MICHIGAN DEPT OF AGRICULTURE

$P$ D BOX 30017

5 LANSING $373-106088909$

\section{RICEE OAVID}

MICHIGAN DEPT DF AGRICULTURE

$P$ C BOX 30017

LANSING. MI 48909

$517373-1060$

ROB INSON ROGER L

FOOD INSPECTOR

MI CEPT OF AGR I CULTURE

$2322 \mathrm{KNOB}$ HILL 18

517 349-0.529

SARULLO SAM

ASST CHIEF

MICHIGAN DEPT OF AGRICULTURE

P O BOX 30017

LANSING. MI 48909
SCHRANZ BARBARA

INSPECTOR

MICHIGAN DEPT OF AGRICULTURE

PO BOX 30017

LANSING MI 48909

517 373-1060

SHELLEY. CHARLES

MICHIGAN DEPT OF AGRICULTURE $P$ D BOX 30017

LANSING. MI 48909

$517373-1060$

SMITH, KIMBERLY

INSPECTOR

MICHIGAN DEPT OF AGRICULTURE PO BOX 30017

LANSING. MI 48909

$517373-1060$

STEPHENSON MICHAEL K

FOOD SPECIAL IST

MICHIGAN DEPT OF AGRICULTURE 244 MARSHALL STREET

ALLEGAN. MI 49010

616 673-8812

STEVENS KEN

MICHIGAN DEPT OF AGRICULTURE

P O BDX 30017

LANSING. MI 48909

517 373- 1060

TAYLOR JOSALENE

INSPECTOR

MICHIGAN DEPT OF AGRICULTURE $P$ O BOX 30017

LANSING. MI 48909

$517373-1060$

TAYLOR, ROBERT

INSPECTOR

MICHIGAN DEPT OF AGRICULTURE $P$ B BOX 30017

LANSING. MI 48909

517 373- 1060

TESCH CHARLES

INSPECTOR

MICHIGAN DEPE OF AGRICULTURE $P$ B BOX 30017

LANSING. MI 48909

$517373-1060$

TITUS, GARY

INSPECTOR

MICHIGAN DEPT OF AGRICULTURE $P$ O BOX 30017

LANS ING. MI 48909

517 373-1060

TOLBERT ER ERNEST
INSPECTOR

MICHIGAN DEPT OF AGRICULTURE PO BOX 30017

LANSING. MI 48909

$517373-1060$

YANSCOTT JUDI

MICHIGAN DEPT OF AGRICULTURE $P$ B BOX 30017

LANSING. MI 48909

$517373-1060$

WARDWELL: LYNN

INSP ECTOR

MICHIGAN DEPT OF AGRICULTURE

$P$ B BOX 30017

LANSING. MI 48909

$517373-1060$ 
WE IONER RICHARD

INICHIGAN DEPT OF AGRICULTURE PO BOX 30017

LANSING MI 48909

517 373-1060

WOJTALA: GERALD

INSPECTCR

MICHIGAN DEPT OF AGRICULTURE PO BOX 30017

LANSING MI 48909

$517373-1060$

ZARA, AMIR

MNSPECTOR $P$ O BDX 30017

LANSING. MI 48909

$517373-1060$

ZORLENE HAROLD

INSPECTOR

MICHIGAN DEPT OF AGRICULTURE

1120 H STATE FAIR

$313368-0280$

\section{CHIPPEWA COUNTY}

DONNELLY, JAMES

INSPECTOR

CHIPPEWA COUNTY

PICKFORD STAR ROUTE

SAULT STE MI 49783

WASHTENAH COUNTY

BENNS DOUGLAS

IN SPECTOR

CONSUMER SERYICES

4133 WASHTENAW/PO BCX 8645

ANN ARBOR MI $4810 \%$

$313971-6054$

BERELS: CHARLEEN

WASHTENAW CO WTS $\&$ MEAS

PO BOX 8645

ANN ARBOR, MI 48107
313 971-6054

PASMAN, NORA J

DIRECTOR

WASHTENAW CO WEIGHT SEMEASURES

P O BOX 8645

ANN ARBCR MI 48107

$313971-6054$

CITY: DEARBORN

CLARK, RODNEY

ORDINANCE ENFORCEMENT DIVISION 2661 GR EENF IELD

DEARBORN, MI 48120

313 943-2294

DAWOY JESS I 2661 GREENFIELD

DEARBOR N. MI 48120

$313943-2294$

\section{CITY: FLINT}

ROBBINS, JOHN H

WEIGHTS $E$ MEASURES INSPECTOR

420 E BLVD DR

FLINT. MI 48503

$313766-7449$
REINHART, AL

INSPECTOR

DEPT OF BLDG SAFETY 6 DEVEL

$119 N$ WASHINGTON SC ANNEX

LANSING. MI 48933

$517483-4386$

MINNESOTA

STATE

ADAMS, BRUCE

WEIGHTS $\&$ MEASURES DIV

STATE OF MINNE SOTA

5202148 TH ST W

APPLE VALLEY. MN 55124

$612341-7200$

MBR

NCWM

ANDERSON JEFF

WEIGHTS \& MEASURES DIV

STATE OF MINNE SOTA

BOX $404 A$ RT 2

SPICER, MN 56288

APP EL HICK. JOE

WEIGHTS $\&$ MEASURES DIV STATE OF MINNE SOTA

1025-8TH ST

PINE CITY MN 55063

$612341-7200$

BAKER LEE

WEI GHTS E MEASURES DIV

STATE OF MINNESOTA

4139 UGSTAD RD

DÚLUTH MN 55811

MBR

NCHM

BLACIK. MICHAEL

HEIGHTS $\&$ MEASURES DIV

STATE OF MINNE SOTA

2277 HIGHHAY 36

ST PAUL: MN 55113

8ORCHARDT N NORM

WEIGHTS $\&$ MEASURES DIV

STATE OF MINNE SOTA

4161 COL ORADO ST SE

612 341-7280

BUCCELLI MARK

HEIGHTS $\&$ MEASURES DIV

STATE OF MINNE SOTA

16312 FLAG STAFF

ROSEMOUNT. MN 55068

$612341-7200$

MBR

NCWM

CLARK, KATHLEEN

HEIGHTS $\&$ MEASURES DIV STATE OF MINNESOTA

2277 HWY 36

ROSEVILLES MN 55113

DONNAN. JOSLYN

METROLOG IST

STATE OF MINNESOTA

2277 HWY 36

ROSEVILLE. MN 55113

$612341-7200$

HAGEN LYNN

WEIGHS \& MEASURES DIV STATE OF MINNESOTA ROUTE 1 WATSONE MN 56295

$612341-7200$ 
HAMMEL BURT STATE OF MINNESOTA RT 1 BOX 208

MILACA MN 56353

HANCZEL — $F$

HEIGHTS $E$ MEASURES DIV

2277 HIGHWAY 36

ROSEVILLE MN 55113

$612341-7200$

HARR I S, GEORGIA

METROLOGIST

STATE OF MINNESOTA

2277 HI GHWAY 36

RO SEVILLE MN 55113

HARRISON, JULIE

WEIGHTS MEASURES DIV

STATE OF MINNESOTA

2277 HIGHWAY 36

ROSEVILLES MN 55113

HEOLUND - RAY

WEIGHTS $E$ MEASURES DIV STATE OF MINNESOTA

224 SO SHERHODD

THIEF RV MN 56701

$612341-7200$

HEDMAN A JAMES

HEIGHTS $\&$ MEASURES DIV STATE OF MINNESOTA

1007 TOPPING

ST PAUL MN 55103

JACCBSON: LARRY

HEIGHTS MEASURES DIV STATE DF MINA

S
SKYLINE M M 56001
$6121-7200$

JOHNSON RICHARD WEIGHTS \& MEASURES DIV

1246 DRAPER

ROSEVILLE MN 55113

612 341-7200

JORGENSEN. GORDEN

WEIGHTS \& MEASURES DIV STATE OF MINNESOTA

612 DONITA

MARHSALL MN 56258

$612341-7200$

JUNKER, DALE

WEIGHTS $\&$ MEASURES DIV STATE OF MINNESOTA

RT 1 BOX 115

CASS LAKE MN 56633

KOETS DAVID

WEIGHTS \& MEASURES DIV STATE OF MINNESOTA

RR 2 BOX 54

MCGRATH. MN 56350

$612341-7200$

LATTIMORE DENNIS DS DIV

STATE.OF MINNESOTA

2400 CROMWELL CIRCLE

MOORHEAD MN

$612341-7200$
MACDONAL D, GEORGE

SUPERVISOR WEIGHTS E MEASURES

STATE OF MINNESOTA

2277 HIGHAY 36

ST PAUL: MN 55113

MAGAARD,PAUL

WEIGHTS $\&$ MEASURES DIV

STATE OF MINNE SOTA

ROUTE 2

RUSHFORD MN 55971

$612341-7200$

MALOTI ELDEN

HEIGHTS E MEASURES DIV

STATE OF MINNESOTA

724 FIRST ST SE

LITTLE FALLS. MN 56345

$612341-7200$

MENK ROGER

WEIGHTS \& MEASURES DIV

STATE OF MINNE SOTA

431 P IERCE AVE

$612341-7200$

MEUWISSEN, VINCENT

HEIGHTS 6 MEASURES DIV

STATE OF MINNESOTA

2277 HIGHWAY 36

ST PAUL. MN 55113

$612341-7200$

MULLENMA ST ER SHERRILL

WEIGHTS \& MEASURES DIV

STATE OF MINNESOTA

RI 2 BOX 861

CAMBRIDGE: MN 55008

$612341-7200$

NELSONE DENNIS

HEIGHTS \& MEASURES CIV

STATE OF MINNESOTA

5257-4TH ST NE

COLUMBIA HEIGHTS。 MN 55421

$612341-7200$

PLYMATE, ELDEN

REGIONAL SUPERYISOR

STATE OF MINNESOTA

2277 HIGHWAY 36

ST PAUL. MN 55113

$612341-7200$

\section{SANDMANN, JAMES}

WEIGHTS \& MEASURES DIV

STATE OF MINNESOTA

RR 1

MORGAN, MN 56266

$612341-7200$

SEXTON, MYRON

WEIGHTS \& MEASURES DIV

STATE OF MINNESOTA

819 DIVISION STH

FARIBAULI MN 55021

$612341-7200$

SKLUZACEK, EDWARD P

DIRECTOR

HTS AND MEASURES DIVISICN

MBR

2277 HI GHWAY 36

PAUL MN 55113

STAPLES, DONALD

WEIGHTS 6 MEASURES DIV

STATE OF M INNESOTA

BoX 601

STARBUCK 341 MN 56381 
SUTTCNE TIM

IGHTS \& MEASURES DIV

STATE OF MINNESOTA

RT 1 BOX 175

LECENTRE MN 56057

VOKCVAN - FRED

WEIGHTS \& MEASURES DIY

STATE DF MINNESOTA

1902 ANDER SON RD

DUEUTH, MN 5 :

WEISS, HOWARD $R$

ME TROLOGIST EMERITUS

STATE OF MINNESOTA

7609 STEVENS AVE

MINNEAPOLIS. MN 55423

$612869-7462$

YOUNG EVA

WEIGH'S E MEA SURES DIV

STATE OF MINNESOTA

2277 HI GHWAY 36

ROSEVILLE: MN 55113

CITY: MINNEAPOLIS

BERGQUIST, JOHN A

DIR LICENSES \& CONSUMER SER

MBR

CITY OF MINNEAPOLI

MINNEAPDLISD MN 55415

MONCUR JNAMES J

DEPT LICENSES \& CONSUMER SVCS

CITY HALL RM 101 A

MNNEAPCLIS: MN 55415

$612348-4283$

MI S S I S SIPP I

STATE

ALLGOOD CRAIG

MS DEPT OF AGRIC \& COMMERCE

1551 BUSCH DAIRY ROAD

LAURER: MS 39440

$601649-1427$

BLACK, WESLEY

PULPHOOOD

MS DEPT OF AGRIC \& COMMERCE

1019 SOUTH JACK SON EXT

BROCKHAVEN MS 39601

$601835-1472$

ELDRIDGE WILLIAM P MS CEPT OF AGRIC \& COMMERCE P O BOX 1609

JACKSON. MS 39215

FREEMAN MARK D

EXECTIVE ASSISTANT

MISS DEPT OF AGRI \& COMMERCE

PO BOX 1609

JACKSON: MS 39205
$601354-6295$
HARDY JOE

OIRECTOR REGULATORY SERYICES

MS DEPT OF AGRIC \& CCMMERCE PO BOX 1609

$J A C K S O N=$ MS 39215
$601359-3637^{3}$

HOWARD。 REED

MIVESTOCK SCALES \& CCMMERCE ROUTE I BOX II

PRAIRIE, MS 39756

$601494-1634$

HOWELL, RALPH

CHIEF GRAIN INSPECTCR

MS DEPT OF AGRIC \& COMMERCE

PO BOX 670

PASCAGOULA. MS 39567

601 762-8141

HUTSON MI ICHEAL

MIVESTOCK SCALES E COMMERCE ROUTE 4

MORTDNE HS 39117

$601537-3463$

LANGSTON EDD

INTERMED IATE SCALES

MS DEPT OF AGR IC \& CCMMERCE PO BOX 67

PRENTISS, MS 39474

$601792-5083$

PER SON BILL

MS DEPT OF AGR IC \& COMMERCE 608 OAK AVE

COL UMBIA MS 39429

$601736-3701$

PROCTOR, JANICE

BIOLOGIST MOISTURE METER LAB MS DEPT OF AGR IC E CCMMERCE PO BOX 351

MERIGOLL D MS 38759

RICE: DAVID

MS DEPT OF AGRIC \& CCMMERCE 204 COTTON ST

ITIA BENA. MS 38941
601 254-7428

ROBBINS RUSTY

MSUCK SCALES OF AGR IC \& CCMMERCE 5840 RIDGEWOOD RO APT H-3

JACKSON. MS 39211

601 957-1987

SLOAN RUSSELL

INTERMED IATE SCALES

MS DEPT OF AGR IC \& CCMMERCE 109 WOODLAND HEIGHTS ABERDEEN: MS 39730 $601369-2853$ SPENCER, JAMES H MISS DEPT OF AGRI \& COMMERCE PO BOX 1609

JACK SON: MS 39205
601 359-3648

SWAIN. HERBERT

MOIISTURE METER FIELDMAN

MS DEPT OF AGR IC \& CCMMERCE

308 SOUTH SECONO

CLEVELAND. MS 38732

$601843-2672$ 
IHCRNTON BUDDY

INTERMEDIATE SCALES

1202 YALE ST

CLEVELAND MS 38732

TORRENCE E ERVIN D

METROLOGIST PO BOX 1043/ALCORN STATE UNIV LORMAN MS 39096

601 B77-3802

TRIPLETT, RANOY

INTERMEDIATE SCALES

MS DEPT OF AGRIC E COMMERCE

PO BOX 32

THOMASTCWN MS 39171

$601289-7722$

HELCH. TIMMY

MILK TANK CALIBRATICN

MS DEPT OF AGRIC \& COMMERCE

1535 HI GHHAY 471

BRANDON. MS 39042

$601829-1315$

WINDHAM GEORGE

PULPHOOD OE AGRIC \& COMMERCE

517 BRO ADWAY APT

WISEMAN, PAUL

PULPWOOD

MS DEPT OF AGRIC \& COMMERCE

621 PARK OR

OXFCRD MS 38655

$601234-4573$

JR I

STATE

ALBERS ROBERT

CHIEF INSPECTCR RT 2

HALF WAY. MO 65663

417 445-2552

ALLEN. ALBERT

CHIEF INSP PETROLEUMIPROPANE

PO BOX 630

JEFFERSCN CITY, MO 65101

BADGER SANDY

DEPI AGRI DIY WEIGHTS \& MEAS

CENTERTOWN, MO 65023

$314584-3429$

BALLE MIKE

INSPECTCR DIV WEIGHTS \& MEAS

RT 3 BOX 100

JASPER, MO 64755

$417682-2026$

\section{BARROWS, LESTER H}

DIV OF WEIGHTS AND MEASURES

P O BOX 630

JEFFERSON CITY. MO 65102

MBR

MBR

NC HM

MBR

MCWM

MCR

940 FANNETTA

DEXTER. MO 63841

INSPECTOR DIV WTS $\&$ MEASURES

DEPT AGRI DIV WTS \& MEASURES

\section{CROSS: FLORENCE}

NCWH

$314751-4278$
CAPPS NORMAN

OEPT AGR I DIV WTS \& MEASURES

$314624-2163$

COOK RICK

ROUTE I BOX 31

CLINTON. MO 64735

$816885-4350$

CREASON. TERRY

INSPECTOR

RR 2 BDX $47 A$

ORR ICK. MO 64077

$816496-3722$

INPPECTIR DIV WEIGHTS \& MEAS 4143 E PENROSE PO BOX 21370

314 LOUIS MO 63115

DRAKE. TIMOTHY

INSPECTOR

DEPT AGRI DIV WTS $\varepsilon$ MEASURES

108 WICKER WOOD

SIKESTON MO 63801

$417471-4579$

ERVIN. KAREN

INSPECTOR

DEPT AGR I DIV WEIGHTS \& MEAS

104 CAMELL IA

CAPE GIRARDEAU. MO 63701

MBR

MBR

NCWM

MBR

MCRM

MBR

FISH, RUSSELL

INSPECTOR DIV WTS \& MEASURES

RT 1 BOX 204

PUXICO. MO 63960

$314222-3869$ 
FLETCHER, MARTHA

INSPECTCR

DEPT AGRI DIY WEIGHTS \& MEAS $413 \mathrm{~N}$ JEFFERSON

VANDAL IIA. MO 63382

$314594-3750$

FRITTS, WAYNE

INSPECTCR DIV WEIGHTS \& MEAS $B 0 \times 358$

HAYNESVILLE, MO 65583

$314774-5542$

GRAY. CHARLES H

INSPECTOR

DEPT AGRI DIV HTS \& MEASURES

PO BOX 326

BOURBON. MO 65441

$314732-5537$

GREENWELL GRANVEL

INSPTECTOR DIV WTS \& MEASURES

POARY CITY, MO 64763

GREENWELL • STEVE

INSPECTOR

DEPT AGRI DIV WTS E MEASURES

810 WIL DWOOD DR FTI

JEFFERSON CITY. MO 65101

MARDING JAMES

INSPTECTQR DIV WTS \& MEASURES

GENERAL DELIVERY

KOELTZTCWN MO 65048

$314728-6494$

HAYESS RCNALD G

DEPT AGRI DIV WTS E MEASURES

PO $80 X \div 30$

JEFFERSCN CITY. MO 65102

HEINEY, DOYLE

INSPECTCR

DEPT AGRI DIV WTS \& MEASURES

ROUTE 2

SUMMERSVILLE. MO 65571

$417932-4486$

HELLEQCENE

DEPTECTOR DIV HTS \& MEASURES

4907 VALLEY LANE

S16 233-07C9 64503

HOLT: FRED

INSPECTCR DIV WEIGHTS \& MEAS

MBR

RT RARIS MO 64645

$816748-4238$

HOOKER。 RON

PROGRAM SUPERVISOR

OIVISION OF WEIGHTS \& MEASURES

PO $80 X \quad 630$

JEFFERSON CITV. MO 65102

HUGHES, SCOTT

INSPECTCR

DEPT AGRI DIV WTS E MEASURES

JEFFERSCN CITY. MO 65101

MBR
NCWM

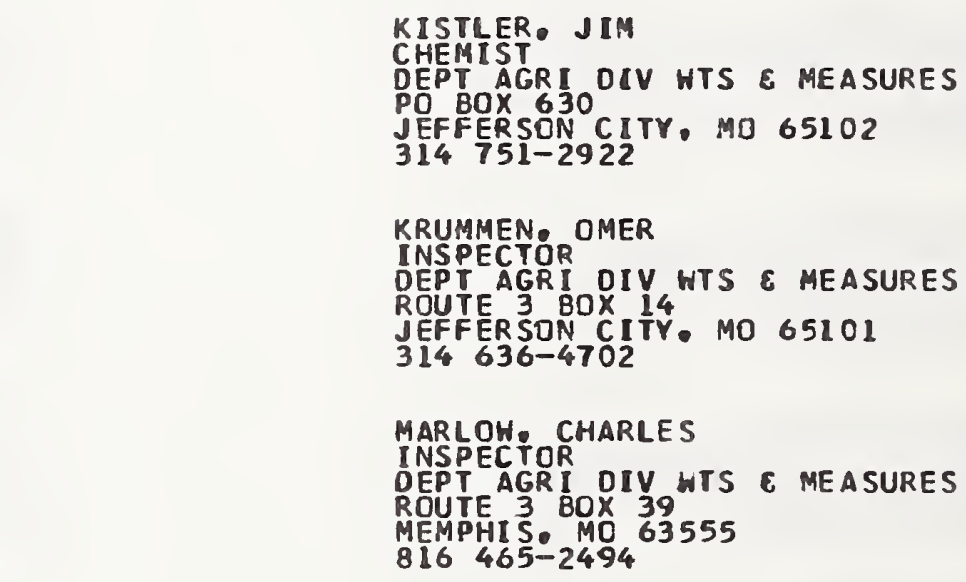

MASSMAN。 CAROLYN

PETROLEUM IAB TECHNICIAN

DEF AGRI DIV WTS E MEASURES

PO BOX 630

JEFFERSON CITY. MO 65102

$314751-2922$

MOORE STEVE

INSPECTOR OIV HTS \& MEASURES $B O X 713$

STEELYILLE MD 65565

PENBERTHY, CHARLES

INSPEECTOR OIV WTS \& MEASURES

BOX $239 A$ UPPER PLATTIN RD

DESOTO. MO 63020

$314586-7142$

PITTSENBARGER, RICHARD

INSPECTOR

DEPT AGRI DIV WTS \& MEASURES

RR 2 BOX 55

MAYSVILLE. MO 64465

$816449-5578$

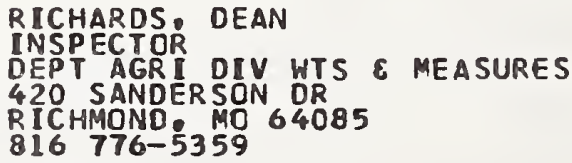

RICHARDS, DEAN

INSPECTOR

DEPT AGRI DIV WTS \& MEASURES

420 SANDER SON DR

RICHMOND. MO 64085

$816776-5359$

MBR

ROBERTS, BILL

INSPECTOR DIV HTS \& MEASURES

RT 4 BOX

WAYNESVILLE MO 65583

ROBINSON: JAMES $V$

INSPECTOR

DEPT AGR I DIV WTS $\&$ MEASURES

PO BOX $166^{\circ}$

PARMA. MO 63870

$314357-4681$ 
ROBINSON JR, FRED

DEPT AGRI DIV WTS G MEASURES

RR 5 BOX 127

FESTUS MO 63028

ROGERS TIM

DEPTECGRI DIV HTS 6 MEASURES ROUTE 1 BOX 229

BILLINGS MO 65610

$417-744-2046$

SHOUSE, DAVID

CHEMIST ARI DIV WTS \& MEASURES PO BOX $630^{\circ}$

JEFFER SON CITY. MO 65102

SKINNER RAY

INEPTECTGR DIV WTS G MEASURES

MBR O BOX 881

CAMDENTON MO 65020

$314346-4718$

STAPLES RICHARD

DEPT AGRI DIV WTS \& MEASURES

BOX 39

EMINENCE, MO 65466

SHOPE CHARLES 1

INSPECTCR

DEPT AGRI DIV HTS \& MEASURES

ROUTE 3 BOX 112

RICH HILL MO 64779

$417395-4134$

THOMAS SOHNNIE J CHIEF NSPECTOR
MO DEPT AGRI WEIGHTS \& MEASURE
ROUT 2 BOX 225

MARSHFI ELD MO 65706

$417468-3751$

MBR

TIPTON

INSPECTCR

DEPT AGRI DIV MTS \& MEASURES

RR 1 BOX 43

NEWTOWN MHC 64667

$816794-2913$

TOLEERS ALLEN

DEPT ÁGRI DIV HTS \& MEASURES

1000 MADISON

816 $827-5251$

IROUTT RON

$\mathbf{R}_{\mathbf{R}} \mathbf{R}$

KNOX CITY. MO 63446

$816434-5230$

UP SCHULTE, KEVIN

PETROLEUM LAB TECHNICIAN

DEPT AGRI DIV WTS E MEASURES

PO BOX 630

JEFFERSCN CITY. MO 65102

$314751-2922$

WEIBLE ROBERT

INSPECTOR

DEPT AGRI DIV WTS \& MEASURES

232 JOHN ST

TROY. MO 63379

$314528-8395$

MBR

MBR
WHITE RQBERT

INSPECTOR

DEPT AGRI DIV WTS \& MEASURES ROCKAHAY BEACH. MO 65740

$417561-4755$

HITTENBERGER R ROBERT

METROLOGIST

MO DEPT OF AGR ICUL TURE

PO BOX 630

JEFFERSON CITY. 19065102

$M B R$

NCWM

$314751-3440$

HOOD. SIDNEY

INSPECTOR

DEPT AGRI DIV HTS \& MEASURES

MAD ISON. MO 65263

$816291-4982$

ST LOUIS COUNTY

ANKER MERLE J

SUPERVISOR

OFFICE OF WEIGHTS \& MEASURES

MBR 7900 FORSYTH BLYD

CLAVTON: MO
$314889-2079$

NCWM

\section{CITY: KANSAS CITY}

ALLEN, WENDELL L

PRÓGRAM MGR HTS $S$ MEASURES

KANSAS CITY MISSOUURI HEALTH DE

1423 E INWOOD BLVD

KANSAS CITY MO 64109

WALKER: TIMOTHY J CITY OF KANSAS CITY AISSOURI 13 EAST 31 ST MO
KANSAS C ITY MO
$816274-1437$

MCER

MONTANA

NCWM

NCWM

STATE

BURNS: JAMES E

BUR OF WEIGHTS $E$ MEASURES

1424 9TH AVE CAPITCL STATION

HELENA. MT 59620

406 449-3494

FRICKEL D MAXINE

BUR OF HEIGHTS \& MEASURES

DEPT OF COMMERCE

1424 9TH AVE CAPITCL STATION

HELENA: MT 59620

$406449-3494$

LANTRY. WILLIAM E

BUR OF WEIGHTS $\&$ MEASURES

DEPT OF COMMERCE

1424 9TH AVE CAPITOL STATION HELENA: MT 59620

$406449-3494$

MELDY STEPHEN H

ADMINISTRATOR

BUREAU OF WEIGHTS $\&$ MEASURES

MBR

1424 9TH AVE

HELENA. MT 59620

$406444-3164$

NISBET DANIEL $K$

DEPUTY SEALER

DIY 24 OTHE AVE

HELENA: MT 59620

$406444-3164$ 
PAGE ALFRED

BUR OF WEIGHTS \& MEASURES

1424 9TH AVE CAPITOL STATION HELENA MT 59620

$406449-3494$

SCHUBERT JERRY

BUR OF HEIGHTS \& MEASURES

DEPT OF COMMERCE

14249 TH AVE CAPITOL STATION

HELENA. MT 59620

THCMPSON, JERRY

BUR OF WEIGHTS \& MEASURES

DEPT OF COMMERCE

I424 9TH AVE CAPITOL STATION HELENA $40649-34949620$

\section{HAYLETT BH RAY E MEASURES \\ DEPY OF COMMERCE \\ 14249 TH AVE CAPITOL STATION HELENA: MT 59620 \\ $406449-3494$}

\section{STATE}

ARNER, SCOTT

AGR INSPECTOR 2 SASKAWEIGHTS AND MEASURES

301 CENTENNIAL MALL SOUTH

LINCOLN. NE 68509

$402471-4292$

BOSCHUL T DAYID

NEBRASKA WEIGHTS AND MEASURES

301 CENTENNIAL MALL SOUTH

LINCOLN. NE 68509

DEISLEY M MIKE

NEBRASKA HEIGHTS ANO MEASURES

301 CENTENNIAL MALL SOUTH

$402471-4292$

MBR

NCWM

ENGELLAND, TIM

AGR INSPECTOR ?

301 CENTENNIAL MALL SOUTH

L NCOLN NE 6.8509

$402471-4292$

KL IMENT GARY

METROLOGIST

WE I GHTS AND MEASURES

LINCOLN NE 68509

$402471-2176$

LOOCK, RICK

AGR INSPECTOR 3

NEBRASKA WEIGHTS AND MEASURES

301 CENTENNIAL MALL SOUTH

$402471-4292$

MALONE STEVEN A

DIRECTOR

HEIGHTS AND MEA SURE S DIVISION 301 CENTENNIAL MALL S BCX94757 $402471-42926208$

MBR

NCWM

MBR

NCHM

MBR

NCWM

MBR

NCWM

MBR

NCWM

MBR

NCWM
OLSDN: CRAIG

NEBRASKA HEIGHTS ANO MEASURES

$P 080 \times 94757$

$402471-217668509$

RAYBURN. AL

AGR INSP EC TOR 2

NEBRASKA WEIGHTS AND MEASURES

301 CENTENNIAL MALL SOUTH

$402471-42926859$

SHAFER KEN

AGR INSPECTOR 2 TS AND MEASURES

301 CENTENNIAL MALL SOUTH

402 ITI-4292 68509

SIMPKINS, TIM D

A G INSPECTOR 2 E MEASURES

301 CENTENNIAL MALL SOUTH

$\angle I N C O L N, N E$

STE INER, RON

AGR INSPECTOR 2

NEBRASKA WEIGHIS ANO MEASURES

301 CENTENNIAL MALL SOUTH

LINCOLN. NE 68509

$402471-4292$

SUITER RICHARD $C$

FIELD SUPERVISOR

NEBRASKA WEIGHTS AND MEASURES P. BOX 94757

INCOLN. NE 68509

TICHOTA, KEN

AGR INSPECTOR 2

NEBRASKA WEIGHTS \& MEA SURES

301 CENTENNIAL MALL SOUTH

LINCOLN, NE 68509

NEVAOA

STATE

BROWN. STEVEN J

WEI GHTS AND MEASURES INSPECTCR NV DEPT OF AGR ICUL TURE

PO BOX 630

ELKO. NV 89801

COYNE, KEVIN

BUREAU OF WEIGHTS \& MEASURES

NV DEPT OF AGR I CUL TURE

MAIL ROCM COMPLEX

LAS VEGAS, NV 89158

DONDERO, PETER

BUREAU OF WEIGHTS \& MEASURES

NV DEPT OF AGR ICUL TURE

$P$ BOX 630

ELKO NV 89801

FULLER. RAY

BUREAU OF WEIGHTS $\&$ MEASURES

NV DEPT OF AGR ICUL TURE

$P Q B O X 11100$

RENO NV 89510 
HEADRICK HALTER

METROLOGIST

NV DEPT OF AGRICULTURE

$P$ BOX 11100

RENC $N$ NV 89431

HOGANSEN EO

NV DEPT OF AGRICULTURE

MAIL ROCM COMPLEX

LAS VEGAS, NV 89158

$702386-5256$

JONES, FRANK

BUREAU OF WEIGHTS \& MEASURES

NV DEPT OF AGRICULTURE

P O BOX 11100

RENO NV 89510

702 789-0166

LARRENETA. MARTIN

BUREAU CF WEIGHTS \& MEASURES NV DEPT OF AGRICULTURE

$P$ BOX 422

WINNEMUCCA NV 89445

$702623-3183$

MARTIN JR, HAROLO D

WEIGHTS \& MEASURES INSPECTOR

NEVAOA DEPT OF AGRI CULTURE

STATE MAILROOM COMPLEX

LAS VEGAS NV 89158

$702386-5256$

\section{MCCREA. BILL}

SEN IOR INSPECTOR

NV DEPT OF AGRICULTURE

MAIL ROCM COMPLEX

LAS
7026 E $386-5356$

OLSEN, TED NV DEPT OF AGRI CULTURE

$P$ BOX 11100

RENC NV 89510

PENNINGTON, KNUTE D

SUPERVISOR HEIGHTS E MEASURES NE VAOA DEPT DF AGRI CULTURE

PO BOX 11100

RENO. NV 89510

$702789-0166$

SMIGEL, THOMAS E

REGIONAL COORDINATOR

NEVAOA DEPT OF AGRICULTURE

STATE MAIL ROCM COMPLEX

LAS VEGAS: NV 89158
$702386-5256$

SMITH, ELOYN

NV DEPT OF AGRICULTURE

$P$ O BOX 11100

RENO NV 8951

\section{HAMPSHIRE}

STATE

COTEERICHARDP

NEW HAMPSHIRE DEPT OF AGRIC

CALLER BOX 2042

CONCORD NH 03301

$603271-3700$
FOSTIER THOMAS J

INSPECTOR

CALLER BOX 2042

CONCORD. NH 03301

$603271-3700$

GRENIER MICHAEL F

METROLLOGIS

NEH HAMPSHIRE DEPT OF AGRIC

MBR

CALLER BOX 2042

CONCORO

HOWARD ROY

DIRECTOR

NEW HAMPSHIRE DEPT CF AGRIC

CALLER BOX 2042

CONCORO. NH 03301

$603271-3700$

MASER, KARL R

INSPECTOR

NEW HAMPSHIRE DEPT OF AGRIC

CALLER BOX 2042

CONCORO NH 03301

$603271-3700$

PEARL - HENRY

INSPECTOR

NEW HAMPSHIRE DEPT OF AGRIC

CALLER BOX 2042

CONCORD: NH 03301

$603271-3700$

WENTWORTH• JEFFREY

INSPECTOR HAMPSHIRE DEPT OF AGRIC

CALLER BOX 2042

CONCORO. NH 03301

$603271-3700$

YOUNG. KEV IN

INSPECTOR

NEW HAMPSHIRE DEPT OF AGRIC

CALLER BOX 2042

CONCORD: NH 03301

$603271-3700$

MBR

CITY: NASHUA

MARQUIS DENNIS

MARQULS OFF WEIGHTS $\&$ MEASURES

NASHUA. NH 03060

MBR

NCGM

NEW JERSEY

STATE

ALL TOBELLI • OINO A

WTS \& MEAS INSPECTCR II

N. WEIGHTS \& MEASURES

187 WEST HANOVER ST

TRENTON. NJ 08625

$609292-4615$

BALL: DONALO J

WTS \& MEAS INSPECTCR I

OFFICE OF WEIGHTS AND MEASURES

MBR

187 WEST HANOVER ST

TRENTON. NJ
$609292-4615$

BISIGNANO, CARMELLO

WTS \& MEAS INSPECTCR II

OFFICE OF WEIGHTS AND MEASURES

187 WEST HANOVER ST

TRENTON. NJ 08625

609 292-4615 
BONAN. WILLIAM J

WTS E MEAS INSPECTOR I

187 HANOVER ST

TRENTON. NJ 08625

$609292-4615$

CARLISI A ANGELO

WTS $E$ MEAS INSPECTOR III

OFFICE OF HEIGHTS AND MEASURES

187 WEST HANOVER ST

TRENTON. NJ 08625

$609292-4615$

CONRAD JR CARL $P$

CHIEF SUPERVISOR AND MEASURES

MBR

187 HANOVER ST

TRENTON. NJ 08625

$609292-4615$

D ERRICO. PASQUALE

INSPECTOR III E PUBLIC SAFETY

187 HANOVER ST

TRENTON NJ 08625

$609292-4615$

GERVASIO, VICTOR

WTS E MEÁS INSPECTOR III

OFFICE OF WEIGHTS AND MEASURES

187 HEST HANOYER ST

TRENTON, NJ 08625

$609292-4615$

HEYDT RICHARD C

WTS \& MEAS INSPECTOR II

OFFICE OF WEIGHTS AND MEASURES

187 HEST HANOVER ST

TRENTON: NJ 08625

$609292-4615$

KELLER HARRY W

SUPERVI SOR

OFFICE OF WEIGHTS AND MEASURES

187 WEST MANOVER ST

TRENTON, NJ 08625

$609292-4615$

KELLY, THOMAS $W$

STATE SUPERINTENDENT

STATE OFFICE OF WEIGHTS $\&$ MEAS 187 WEST HANOVER ST

TRENTON. NJ 08625

$609292-4615$

KROL. RICHARD C

WTS \& HEAS INSPECTOR

OFFICE OF WEIGHTS AND MEASURES

187 HEST HANOVER ST

TRENTON. NJ 08625

$609292-4615$

LORD. RGNALD H

SUPV OF LUMBER INSPECTICNS

OFFICE OF HEIGHTS AND MEASURES

187 WEST HANOVER ST

TRENTON. NJ

MASITTI. MARID

WTS \& MEAS INSPECTOR I

187 WEST HANCVER ST

TRENTON. NJ 08625

$609292-4615$

MORRIS CONALD

WTS E MEAS INSPECTORS II

187 HEST HANOVER ST

TRENTON NJ 08625

$609292-4615$
MORRIS. JAMES $P$

OFFICE OF WEIGHTS AND MEASURES

187 WEST HANOVER ST

TRENTON. NJ 08625

609 292-4615

MOSCARELLO- JOHN A

WTS \& MEAS INSPECTCR II I

OFFICE OF WEIGHTS AND MEASURES

187 HEST HANOVER ST

TRENTON. NJ 08625

609 292-4615

MYERS SR HERBERT E

UTS 6 MEAS INSPECTCR II

OFFICE OF WEIGHTS AND MEASURES

TRENTON, NJ 08625

$609292-4615$

NER I - ANTHONY H

WTS \& MEAS INSPECTCR II

OFFICE OF WEIGHTS ANO MEASURES

187 WEST HANOVER ST

TRENTON. NJ 08625

609 292-4615

OGRADY HILLIAM R

HTS \& MEAS INSPECTCR I

OFFICE DF WEIGHTS AND MEASURES 187 WEST HANOUER ST

T.RENTON: NJ

ROMAND, JOSEPH

HTS 6 MEAS INSPECTCRS III

OFFICE OF WEIGHTS AND MEÁSURES

187 HEST HANOVER ST

TRENTON. NJ 08625

609 292-4615

SCARPELLI, FRANK A

SUPY DF TECH SERYICES

OFF ICE OF HEIGHTS ANO MEASURES

187 HEST HANOVER ST

TRENTON. NJ 08625

609 292-4615

MBR

NCHM

SEHGEL D HEREERT

HTS $\&$ MEAS INSPECTCR I

OFF ICE OF WEIGHTS AND MEASURES

187 HEST HANOVER ST

TRENTON. NJ 08625

609 292-4615

SMITH, HAL TER L

WTS \& MEAS INSPECTCR II

OFF ICE OF WEIGHTS AND MEASURES

187 WEST HANOVER ST

TRENTON. NJ 08625

$609292-4615$

TILELLI CARMINE

WTS $\overline{M E A S}$ INSPECTCR II

OFF ICE OF WEIGHTS AND MEASURES

187 WEST HANOVER ST

TRENTON. NJ 08625

$609292-4615$

ZINSKI ROBERT

INSPECTOR III

STATE DFFICE OF WTS \& MEAS

187 W HANO VER ST

$609292-4615$

ATLANTIC COUNTY

GALLO, SAL

ASST SUPT

ATLANTIC COUNTY

1333 ATLANTIC AVE

ATLANTIC CITY. NJ 08401

$609645-7700 \times 4271$ 
HADYKA JOE

SUPERINTENDENT

DEPI OF WEIGHTS

ATLANTICACITY NJ 08401

$609345-6700 \times 2485$

\section{BRGEN COOUNTY}
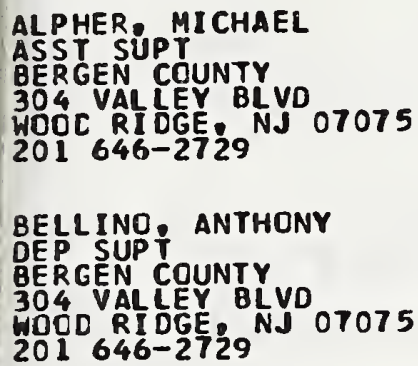

\section{DESTEFAND, JAMES \\ ASST SUPT \\ 304 VALLEY BLVD \\ WOOC RIDGE NJ 07075 \\ $201646-2729$}

MAGUIRE JOHN

BERGEN COUNTY

304 VALLEY BLVO 07075

$201646-2729$

POLLOCK JAMES A

SUPER INTENDENT

BERGEN CTY WTS \& MEAS DEPT

304 VALLEY BLVO

WOOC RIDGE NJ 07075

201 646-2729

RATCLIFFE, RICHARD T

AS ISTANT CTY SUPER INTENDENT

304 VALLEY BLVD

WOOC RICGE. NJ 07075

$201646-2729$

WASSBERG, JOHN

BERGEN COUNTY

304 VALLEY BLVO

WOOC RIOGE NJ 07075

$201646-2729$

\section{BURL INGTON COUNTY}

BORSTAD. RENEE L

ACTNG SUPT

BURLING TON COUNTY

49 RANC CLAS ROAD

MT HOLLY $261-5098$

\section{CAMDEN COUNTY}

\section{CULBERT SCN. ROYDEN}

ASST SUPT

CAMDEN CCUNTY

JEFFERSOLN HOUSE LAKELAND ROAD BLACKWOOD NJ 08012

$609757-8700$

\section{GARVEY, THOMAS C}

SUP ERINTENDEN

JEFFERSCCN HOUSE LAKELAND RDAO

609 757-8700 08012
MILLS FRANK

CAMDEN COUNTY HEIGHTS 6 MEAS

LAKELANO RD

BLACKHOOD NJ 08012

$609757-8700$

PARKER - ANDREW F

DEPUTY SUPER INTENOENT

CAMDEN CDUNTY

JEFFERSON HOUSE LAKELAND ROAD

BLACKHOOD NJ 08012
$609757-8700$

CAPE MAY COUNTY

TEENEY JAMES $J$

SUPER INT ENDANT

CAPE MAY COUNTY HGHTS E MEAS

LIBRARY BLDG MECHANIC ST

CAPE MAYE NJ 08210

$609465-7111 \times 398$

\section{CUMBERLAND COUNTY}

FRANKS • GEORGE S

COUNTY SUPERINTENDENT

DEPT WTS $E$ MEAS \& CCNS PROT

MBR

788 E COMMERCE ST

BRIDGETON NJ 08302

$609451-8000 \times 370$

GAMBA. FRANK

ASST SUPT

CUMBERLAND COUNTY

788 E COMMERCE ST

BRIDGETON. NJ 08302

$609451-8000 \times 369$

TOWNSEND,JR. JONAS E

ASST SUPT

CUMBERLAND COUNTY

788 E COMMERCE ST

BRIDGETON. NJ 08302

$609451-8000 \times 369$

ESSEX COUNTY

LEGGE JR, JOHN

ASST SUPT

ESSEX COUNTY

900 BLCOMF IELD AVE

VERONA: NJ 07044

PENHALE, EOWARD $R$

SUPT

ESSEX COUNTY

900 BLOOMFIELD AVE

MBR

NCWM

VERONA: NJ 07044
$201266-1560$

IL SON ERNEST

ASST SUPT

ESSEX COUNTY

900 BLOUMFIELD AVE

VERONA: NJ 07044

201 266-1560

GLOUCESTER COUNTY

CAL I RUSSELL

ASST SUPT

CD BLDG 49 WOOD ST

WOODBURY NJ 08096

$609853-3358$

SILVESTRO, JOSEPH

SUP ERINTENDENT

GLOU CO WT SEMEAS DEPT

49 WOOD ST COUNTY BLDG

HOODBURY NJ 08096

$609853-3358$
MBR

MBR

NCWM

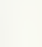


HUDSCN COUNTY TURNER • FRANK M

HUDSON COUNTY

595 NEWARK AVE

JER SEY CITYg NJ 07306

HUNTERDON COUNTY

YANCY, WILLIAM

SUPERINTENDENT

HUNTERDCN CO DEPT WTS $E$ MEAS

HALL OF RECORDS

FLEMINGTON, NJ 08822

\section{MERCER COUNTY}

MCCABE, DONALD $R$

SUPT OF WEIGHTS AND MEASURES

CDUNTY OF MERCER

640 S BROAD ST

TRENTON NJ 08650

\section{MIDDLESEX COUNTY}

CHOHAMIN NUDOHN M

MIDDLESEX CTY DEPT WTS 6 MEAS

MBR

NORTH BRUNSWICK. NJ 08902
$201745-3298$

MONMCUTH COUNTY

\section{ALLEN JOOHN \\ MONMOUTH COUNTY \\ 40 MONUMENT ST \\ FREEHOLD? NJ 07728}

BENNETT III. JOHN

MONMOUTH COUNTY

40 MONUMENT ST

FREEHOL D
201 N
$21-7362$

\section{CDOK. RAYHOND}

ASST SUP

MONMOUTH COUNTY

40 PONURENT ST

FREEHOL D 2 N O7728

\section{DOGGETTS WILLIAM}

MONMOUTH COUNTY

40 MONUAENT ST

$201431-7362$

DOX WILLIAM G

SUPERINTENDEN

MON MOUTH COUNTY DEPT WTSEMEAS

MBR

\section{FREEHOL D NJ 07728}

KEENAN JR, CHARLES D

ASS I STANT SUPER INTENDENT

MONMOUTH CNTY WEIGHT $E$ MEAS

40 MONUMENT ST

FREEHOL D NJ 07728
PELUSO BENJAMIN S

DEPUTY SUPERINTENDENT

MONMOUTH COUNTY

40 MONUMENT ST

FREEHOLD ${ }^{2} J^{\circ} 07728$
$201431-7362$

\section{MORRIS COUNTY}

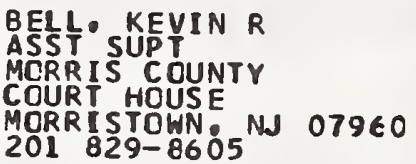

CIFRESE VITO

ASSISTANT SUPERINTENOENT

MORRIS COUNTY WTS E MEAS

COURTHOUSE

MORRISTOWN $201829-8605$ NJ 07960

CRANE。 WALLACE

ASSI STANT SUPER INTENDENT

MORR IS COUNTY WTS 6 MEASURES COURTHOUSE

MORRISTOWN, NJ 07960

DONNELLY WALTER $H$

MORRIS COUNTY WEIGHTS \& MEAS

COURTHOUSE

MORRISTOWN \& NJ 07960

LORI ANTHONY

DEPUTY SUPERINTENDENT

$M$ C WEIGHTS \& MEASURES

COURTHOUSE

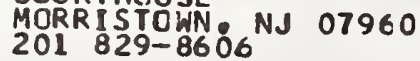

MCCORMICK• ALBERT

ASST SUPT

MORRIS COUNTY

COURT HDUSE

MORRISTOWN NJ 07960

201 829-8605

STPUBLE BENJAMIN

CDUNTY SUPER INTENDENT

DIVISION OF WEIGHT \& MEASURES

MORRIS COUNTY COUR THOUSE

HORRISTOWN NJ 07960

OCEAN COUNTY

BROOKS BART H

ASST SUPT

OCE AN COUNTY

38 HADLEY AV CN 2152

$201929-2166$

BURD JR, MALCOLM R

COUNTY SUPER INTENDENT

DEPT OF WE IGHTS AND MEASURES

38 HADLEY AVE/CN 21 S2

TOMS RIVER NJ 08753

$201929-2166 \times 3257$

HULME, WILLIAM J

DEPUTY SUPERINTENDENT

OCEAN COUNTY

38 HADLEY AV CN 2152

TOMS RIVER: NJ 08753

201929-2166 


\section{SALERNO, ERNEST \\ AS ST SUPT \\ PASSAIC COUNTY \\ 317 PENNSYLVANIA AVE \\ $201881-4373$ \\ VATASIN JOHN \\ SUPERINTENDENT \\ PASSAIC COUNANIA AVE \\ PATERSDN NJ 07503 \\ $201881-4375$}

\section{ALEM COUNTY}

JONES, ROBERT B

SUPER INTENDENT

SALEH COUNTY DEPT WTS \& MEAS

94 MARKET ST

SALEM, NJ 08079

$609935-7510 \times 369$

\section{SOMERSET COUNTY}

DOMBEY DAVIO A

ASST SUPT COUNTY

N BRIDGE \& HIGH STS BOX 3000 SOMERVILLE. NJ 08876

$201231-7125$

HUNT. PETER J

ASST SUPY

SOMERSE T COUNTY

N BRIDGE \& HIGH STS BDX 3000

$201231-7125{ }^{\text {SJ }} 08876$

MAHER, JAMES $G$

SUPERINTENDENT

N BRIDGE E HIGH STS/BOX 3000 SOMERYILLE NJ 08876

$201231-7125$

VROOM. WILLIAM $R$

DEPUTY SUPER INT ENDENT

SOMERSET COUNTY

BOX 3000

SOMERVILLE NJ 08876

SUSSEX COUNTY

DEPUY RONALD J

ASST SUPT

SUS SEX COUNTY

RD 3 BOX 140

NEWTON NJ 07860

$201948-5464$

HEATER。 JERRY $M$

SUPERINTENDENT

SUS SEXC DDEPTOFWE IGHTSEMEASURES

MBR

NEWTON NJ 07860

$201948-5464$

MINAHANE $T$ BRENT

AS ST SUP SUNTY

RD 3 BOX 140

NEWTON: N J 07860

$201948-5464$

PROUT JR JACK

DEPUIY SUPERINTENDENT

SUS SEXCODEPTOFWEIGHT SE HEA SURES

RD 3 BOX 140

NEHTON: NJ 07860

$201948-5464$

\section{UNION COUNTY}

GEHRKE WILLIAM

ASSISTANT SUPER INT ENDENT

UNION COUNTY DIV WEIGHTS-MEAS

$30 O$ NORTH AVENUE E

WESTFIELD NJ 07090

KARAMUS THOMAS

SUP ER INT ENDENT

UNION COUNTY DIV WEIGHTS-MEAS

300 NDRTH AVENUE

WESTFIELD. NJ 07090

$201654-3875$

\section{WARREN COUNTY}

DAVIS J JOHN W W

WEIGHTS E MEASURES

COURTHOUSE

BELVIDERE $475-5087$

PAULIK. EDWARD

ASST SUPER INTENDENT W $6 M$

WARREN COUNTY

WARREN CO COURTHOUSE

BELVIDERE NJ 07823

$201475-5087$

YAN SYCKLE FLOYO

SUPERINTENDENT

WARREN COUNTY

WARREN CO COURTHOUSE

BELVIDERE: NJ 07823

201 475-5087

CITY: ATLANTIC CITY

MBR

NCWM

KELLY JOHN F

SUPER INT ENDENT

DEPT OF WEIGHTS $E$ MEASURES

501 N AL BANY AVE

ATLANTIC CITY. NJ C8401

$609347-5527$

CITY: BAYONNE

ZUBER MICHAEL

ASST SUPT

630 AVENUE $C$

BAYONNE. NJ 07002

$201858-6109$

CITY: BELLEVILLE

LEONARD. EOWARD J

SUP HASHINGTON ST TOWN HALL BELLEVILLE: NJ $071 \mathrm{CO}$ $201759-3399$

CITY: CAMDEN

SMITH. LARRY F

SUPER INTENDENT

415 FEDERAL ST

$609757-7313$ 


\section{CITY: CLIFTCN}

ADELHELMO WILLIAM

SUPT OF MEIGHTS AND MEASURES 900 CEIFTON AVE

CLIFTON: NJ OTO13

CITY: E DRANGE

FRAGOLA, GAETANO

SUPER INTENDENT

E C CITY HALL PLAZA

EORANGE $266-5318$

CITY: ELIZABETH

LATCRRE WILLIAM D

ASST SUP

EL M NFIELD SCOTT PLAZA

$201353-6000 \times 307$

SCHUMACHER HOWARD

CITY HALL ROOH G-3

ELI ZABETH NJ 07201

\section{CITV: HAMILTON}

NAIRA, SAMUEL $R$

SUPT

209 C GREENGOOD AVE CNOO150

HAHILTON. NJ 08650

609 890-3655

CITY: JERSEY CITY

EVANS HILLIAM

8 STR SUPT

$201547-5292$ NJ 07302

GRAJEWSKI,ED

8 ASTT SUPT

201 SERSY CITY, NJ 07302

GRISI LAVID

A ERIE ST

JERSEY CITY NJ 07302

WERMERT PETER A

SUPT WEIGHTS E MEASURES

CITY OF JERSEY CITY

8 ERIE ST

JERSEY CITY, NJ 07302
$201547-5292$

CITY: LINDEN

ESKA。 ALEXANOER

SUPER INTENDENT

CITV OF LINDEN WTS AND MEASURE

MBR

LINDEN NA 07036
$201486-3800$

\section{CITY: NEWARK}

PIZZANO. LOUIS

SUPER INTENDENT WEIGHTS \& MEAS

CITY OF NEWARK

94 WILLIAM STREET

NEWARK: NJ OT102

$201733-6274$

CITY: NORTH BERGEN

OVERMAN. RCBERT $G$

TOWNSHIP DEPT OF $W \& M$

4233 KENNEDY BLVD

NORTH BERGEN. NJ 07047

CITY: NUTLEY

SCHUMAN, WILBUR

SUPER INT ENDENI

TOWN OF NUTLEY

PUBLIC SAFETY BLDG

NUTLEY. NJ 07110

CITY: PATERSON

CAFFREY - WILLI AM

SUPT OF WGTS E HEA SURES

DEPT OF WEIGHTS E MEAS

170 BROAOWAY

PATERSON. NJ 07505

$201881-3730 \times 29$

TORCHIANI. ITALO

DEP SUPT

I76 BROAOWAY

PATERSON NJ 07505

$201881-3730$

CITY: TRENTON

MOTCHNIK WALTER E

SUP ER INTENDENT

WEIGHTS \& MEASURES

319 E ST ATTE ST

TRENTON. NJ 08608

NC

609 989-3282

NEW MEXICO

STATE

BARELA。 ERNEST $S$

INSPECTOR III

NM WEIGHTS \& MEASURES DIV

2604 ALT EC N

AL BUQUERQUE. NM 87107

$505841-6516$

BARNES。 ROMA A

INSPECTOR II

NM WEIGHTS \& MEASURES DIV

402 NEWBY LANE

BLDOMFIELD NM 87413
$505632-3757$

MBR

CASAUS, ROBERT

INSPECTOR I 2604 AZTEC NE MEASURES DIV

ÁLBUQÚERQUE: NM 87107

$505841-6516$

COLLINS. C VICTOR

INSPECTOR IV

NM WEIGHTS \& MEASURES DIV

LAS CRUCES.

505 646-16:16 
CULBERTH LYNN T MBR

INSPECTCR I \& MEASUP.ES DIV NCWM

137 CEDAR

CLOVIS. NM 88101

$505762-1368$

DARBY JAMES $W$
INSPECTOR III
NM HEIGHTS E MEASURES DIV
P O BOX 3170

$P$ O BOX 3170

LAS CRUCES NM 88003

GERK FRED FR

NM DEPT OF AGRICULTURE

PO BOX 3170

LAS CRUCES NM 88003

\section{GOMEZETJOE \\ NMS WEIGHTS \& MEASURES DIV \\ 2604 AZTEC NE \\ ALBUQUERQUE NM 87107 \\ $505841-6516$}

GREENE, CHARLES $H$

DIRECTOR GENERAL SERVICES NM DEPT OF AGRICULTURE

PO BOX 3189

505 CRUCES: NA 88003

HALLE BRIAN

INSPECTCR I \& MEASURES DIV

2604 AZTEC NE

AL BUAUERQUE? NM 87107
$505841-6516$

HURLESS RICK W

INSPECTCR I I MEASURES DIV

ROSWELL. NM 88201

$505625-9114$

LLANEZ JOE P

NM WEIGHTS \& MEASURES DIV

$P$ BDX 3170

LAS CRUCES: NM 88003

MARTIN JIHMY

INSPECTOR I

NM WEIGHTS $\&$ MEASURES DIV

列 585

$505394-2668231$

MENDOZA HILFRED

INSPECTOR IV
NM WEIGHTS E MEASURES DIV
2604 AZTEC NE

AL BUQUERQUE: NM 87107

$505841-6516$

MEYER MARK J

INSPECTCR TRA INEE

NM WEIGHTS \& MEASURES DIV

2604 AZTEC NE

ALBUQUERQUE NM 87107

PERALTA JOHNNY

INSPECTOR I NMEASURES DIV

2604 AZTEC NE

AL BUQUERQUE, NM 87107

$505841-6516$

MBR

MBR

MBR

MBR

MBR

MBR

MBR

MBR
PIERCE DOUGLAS J

INSPECTOR III WEASURES DIV

3907 COUNTRY CLUB DR

FARAINGTON NM 87401

$505327-6496$

NCWM

SCHULAEISTER, RICHARD F

NETROLOGIST DEPARTMENT OF AGRI

MBR

NM DEPARTMENT OF AGRI

LAS CRUCES: NM 88003

SEGOVIAO BENJAMIN M

NM WEIGHTS \& MEASURES DIV

P O BOX 3170

LAS CRUCES NM 88003

SHUGART, JERRY $M$

INSPECTOR I I MEASUS \& MEASURS DIV

ST RT BOX 35-0

CLOVIS NM 88101

STEFFEY MICHAEL $R$

PETROLEUM MEASURE BUREAU CHIEF NM HEIGHTS \& MEASURES DIV

PO $80 \times 3170$

LAS CRUCES NM 88003
505 646-1616

NCWM

UTTON JRE GRION

NM WEIGHTS \& MEASURES DIV

PD BOX 249

ROSWELLL: NM 88201

WEST GARY D

MULTI-PURPOSE BUREAU CHIEF PM WEIGHTS \& MEASURES DIV

LAS CRUCES NM 88003

505 646-1616

NEW YORK

STATE

NCWM

METROLOG IST

NYS BUREAU OF WEIGRTS \& MEAS

BUILDING IA STATE CAMPUS

ALBANY NY 12235

$51845 \%-3449$

BARTFAI: JOHN J

DIRECTOR BUREAU OF WEIGHTS \& MEASURES

1220 WASHINGTON AVE

ALBANY NY 12235

$518457-3452$

DAVIS, DELORES D

WEIGHTS MEASURES SPEC III

BUREAU OF WEIGHTS $E$ MEA SURES

BLOG TA STATE CAMPUS

ALBANY NY 12235

$518457-3452$

FISHMAN。WILLIAM

MBR

WEIGHTS \& MEASURES SPECIALIST 103-00 SHORE FRONT PKWY \#7H ROCKAYAYY PARK. NY 11694

$718945-3806$

NCWM

MBR

MBR

NCWM

MBR

NCWM

NCWM

MBR

NCLM

MBR

MBR

NC GM 
WIPITZZ JAN A

WEIGHTS E MEASURES SPECIALIST 93 WESTERLOE AVENUE

$716461-2259$

LYDEN PETER A

WTS E MEAS SPECIALIST II

SCHENEC TADY NY 12304

$518393-2857$

MURPHY, JAMES J

DIRECTOR MATERIALS BUREAU

NY STATE OEPT OF TRANSPORTATN 1220 HA SHINGTON AVE

ALBANY NY 12332

$518457-3240$

PAUTZ, EOWARD F

WEIGHTS E MEA SURES SPECIALIST

763 THOMP SON STREET

ELMIRA NY 14904

$607732-1605$

SZESNAT • EDWARD

7 PEYER DR

ALBANY
518 S $869-58212205$

TARBOX JOHN

2627 GR ANDV IEW PL

ENOICOTT N NY

YAGUD. IRVING

WTS \& MEAS SPECIALIST I

68-42B 186 LANE

FRESH MEADOWS, NY 11365

$718-969-7619$

\section{ALBANY COUNTY}

GQRMAN. FRANK

DEPUTY DIRECTOR WGTS 6 MEASURE

COUNTY OF ALBANY

COUNTY COURTHOUSE RCOM 40

ALBANY? NY 12

WEAFER KENNETH A ${ }^{A}$ MECTOR WEIGHTS $E$ MEASURES

COUNTY OF ALBANY

COUNTY COURTHOUSE RCCM 40

AL B ANV NV 12207

$518445-7538$

\section{ALLEGANY COUNTY}

CHAMBERLAIN。 ALAN D

DIRECTOR

5 FURNESS ST

BELFAST: NY 14711

716 268-5002

BROOME COUNTY

HEATHERMAN, RICHARD T

DIRECTOR

BRDOME COUNTY WTS \& MEAS

CDUNTY OFF BLOG GVT PLAZA

$607772-2195$ NI 13902

JABLONOWSKI - THOMAS $M$ COOROINAIOR OF CONSUMER AFFAIR

COUNTY OFFICE BLDG

BI NGHAM TON NY 13902

MBR

MBR

MBR
NCWM

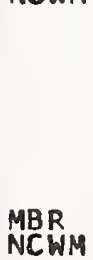

IL SON: WILLIAM J

DIRECTOR OF WTS \& MEASURES

PD EOX 1726 ELM ST

PERU. NY 1297

$518 \quad 643-8536$

COLUMBI A CDUNTV

NEAR HERBERT D

DIRECTOR

184 MAIN ST

PHILMONT NY 12565

$518672-4866$

CORTLAND COUNTY

ARMSTRONG。 GEORGE

DIRECTOR WEIGHTS \& MEASURES

PO BOX 5590

CORTLANDS NY 13045

DELAWARE COUNTY

SCHLAFER, DANIEL

DIRECTOR

RD I BENNETT HOLLOW ROAO

FRANKLIN. NY 13775 
ENNEDY: JOHN T'S \& MEASURES UTCHESS COUNTY 2 MARKET ST

IIUGAKE 14 -6322. NY 12602

SRANKER , NELSON

SENIOR INSPECTOR

JUTCHESS COUNTY

22 MARK ET ST

POUGHKEEPSIE, NY 12601

$914471-6322$

\section{EIE COUNTY}

SYLVES̊ JAMES F

ERIE COUNTY WTS \& MEAS

2380 CLINTON ST

BUFFALD: NY 14225

$116825-1310$

THOMPSON' KENNETH L

OEPUTY COUNTY BUR DF hTS \& MEAS

2380 CLINTON ST

CHEEK NY 14227

SSEX COUNTY

\section{TREADWAY. CHARLES \\ DIRECTOR ROAD - CHILSON \\ ICCNDERCGA NY 12883 \\ 518585 -7909}

RANKLIN COUNTY

GARDNER HARRY R OF HE IGHTS AND MEASURES

89 WEST MAIN STREET

MALCNE, NY 12953

$518483-6767 \times 397$

\section{=ULTON COUNTY}

BOOTH CLINTON L

WEIGHTS $\&$ MEASURES

PO BOX 131 GRAY ROAD

MAYFIELD NY 12117

$518661-5389$

\section{GENESEE CCUNTY}

HAXTON. HILLIAM

OIRECTOR WEIGHTS MEASURES

$362 C$ SOUTH MAIN ST ROAD

BATAVIA. NY 14020

716 343-6943

\section{GREENE COUNTY}

MCOCNALD, FRANK J

DIRECTOR

125 HATER ST

CATSKILL NY 12414
ROCKWELL. WARREN PHIL

DIRECTOR WEIGHTS \& MEASURES

ROUTE $28 \mathrm{~N}$

LONG LAKE. NY 12847

518 624-4169

HERKIMER COUNTY

STARUCK. STEVE

DIRECTOR

HERKI MER NY 13350

$315867-1203$

JEFFERSON COUNTY

MBR

MBR

NCWM

MBR

NCWM

NCWR

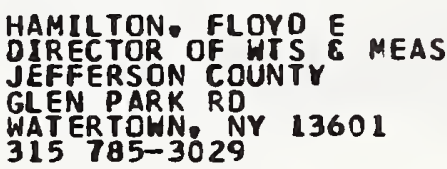

LEWIS COUNTY

PARSELL STEVEN C

DIRECTOR

BOX 381

LYONS FALLS: NY 13368

MBR

LIVINGSTON COUNTY

HEBSTER, DONALD J

TURKEY HILL ROAD

CONESUS. NY 14435

716 346-3162

MADISON COUNTY

WILCOX WILLIAM M

BUREAU OF WEIGHTS \& MEASURES

PO BOX 28 /TIMHERMAN ROAD

CLOCKVILLE NY 13043

$315697-9944$

MBR

NCWM

NCWM

MONROE COUNTY

FORDE CLEVELAND C

DIRECTOR

DEPT WEIGHTS E MEASUR

ROCHESTER NY 14624

$716436-1330$

MONTGOMERY COUNTY

CALELLA JOSEPH

SEALER HEIGHTS \& MEASURES

MONTGOMERY COUNTY

CITY HALI

AMSTERDAM NY 12010

$518841-4322$

MBR

NCWM

MBR

NCWM

NASSAU COUNTY

PICKEN JAMES

COMMISSI ONER

OFFICE OF CONSUMER AFFAIRS

160 OLD COUNTRY ROAD

MINEOLA. NY 11501

$516535-3200$
MBR

NCWM 
LAMONT\& RICHARD

OIRECTÓR OF WEIGHTS \& MEASURES NI AGARA COUNTY

100 DAVISON RCAD

LOCKPORT: NY 14094

$716439-6096$

ONEIDA COUNTY

WHITE\& MARK E

ONE IDA COUNTY GOVT

800 PARK AVE

315 UT ${ }_{3}$ NY -5601501

ZEIGLER, RDBERT J

BUREAU OF WEIGHTS AND MEASURES ONEIDA COUNTY OFF BLDG

UT I CA NY 1350

ONONDAGA COUNTY

BARTLETT ALFRED
OIRECTOR WEIGHTS

OIRECTOR WEIGHTS AND MEASURES

421 MONTGOMERY STIVISICN WTS

SYRACUSE, NY 13202

ONTARIO COUNTY

FOX FRED H

DIRECTOR

WE IGHTS AND MEASURES

3907 COUNTY RD 46

CANANDAIGUA: NY 14424

$716394-7070 \times 2193$

\section{ORANGE COUNTY}

BROWN ECHARDJ

ASST DIR WEIGHTS AND MEASURES

OR CO DEPT WEIGHTS \& MEASURES

99 MAIN ST

GOSHEN, NY 10924

$914294-5151 \times 162$

VAN ZETTA, JAMES A

DIRECTOR

COURT HCUSE ANNEX

GOSHEN NY 10924

\section{ORLEANS CCUNTY}

KLATT RANDALL

DIREC TOR WEIGHTS MEASURES

CRLEANS CGUNTY

ALEILN, NY 1.441 .

71 E 589-7778

\section{CSWEGC COUNTY}

LEARNED, SHAWN

DIRECTOR

7366 PORT ST

PULASKI. NY 13142

$315290-2039$
PEMBERTON, BARRY

OIRECTOR OF WE IGHTS \& MEASURES OTSEGO COUNTY BOX 87

MILFO̊RD. NY 13807

PUTNAM COUNTY

MBR

MBR

NCHM

MBR

MBR

NCWM

MBR

VCWM

D OTTAVIC. VAL

SEALER OF WEIGHTS \& MEASURES PUTNAM COUNTY 2 CQUNTY CENTER RM 304 CARMEL: NY 10512

$914225-3641 \times 211$

RENSSELAER COUNTY

SHEVLIN, LAWRENCE E

RIRECTOR WEIGHTS ANDD MEASURES

1600 7TH AVE-COUNTY OFF BLDG

TRQY NY 12180

ROCKLAND COUNTY

FARKAS JAMES

DIRECTOR COORDINATCR

DEPT OF WEIGHTS \& MEASURES

COUNTY OFF ICEE BUILCING

$914638-5280$

SARATOGA COUNTY

INDIANO. THOMAS M

DIRECTOR

31 WOODLAWN AVE

SARATOGA SPRINGS. NY 12866
518 587-0360

SCHENECTADY COUNTY

BRIGGS, DOUGLAS A

OIRECTOR WEIGHTS \& MEASURES

SCHENECTAOY COUNTY

80 KELLAR AVE

SCHENECTADY. NY 12306

$518382-3297$

SCHOHARIE COUNTY

BERARD. PAUL

DIRECTOR HEIGHTS \& MEASURES

$80 \times 672$

SCHOHAF.IE NY .22157

518 295-8i72

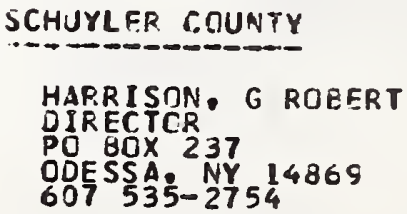

SENECA COUNTY

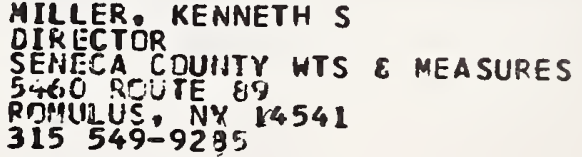

MBR

NChM 
MCMILLAN, ROGER

DIRECTOR RO CO HWY ANNEX

CANTON NY 13617

$315379-2376$

\section{SEUBEN COUNTY}

ABBEY DENNIS

DEPT OF WEIGHTS \& MEASURES

40 E STEUBEN ST

BATH NY 14810

$607376-4949$

\section{NFFCLK CCUNTY}

\section{GARONER, CHARLES A \\ DIRECTOR WTS \& MEASURES COUNTY CTR N COMPLEX VETS HWY HAUPPAUGE NY 11787 \\ $516360-4620$}

\section{ULL IVAN COUNTY}

SMITH GERALD

DIRECTOR

WEIGHTS AND MEASURES

RIVER RCAD

CALLICOON NY 12723

$914887-4569$

IOGA COUNTY

LOUNSBURY, SHELDON L

DIRECTOR

COUNTY

TIOGA CENTER. NY 13845

IOMPK INS COUNTY

EMMICK LOUIS

DIRECTOR

COURTHOUSE ROOM 6

${ }_{607}^{1} \mathrm{~T}^{2} \mathrm{~A}_{4}-5205$

\section{ULSTER COUNTY}

MAYCNE SR, RALPH F

DIRECJOR OF WEIGHTSANDMEASURES

UL STER COUNTY

4514 EBELS CT 12477

$914331-9300$

MBR

NCHM

\section{WARP.EN COUNTY} WERTIME GEORGE W

MBR WARREN COUNTY MUNICIPAL BLDG

LAKE GEORGE NY 12845

$518761-6496$

\section{WASHINGTON COUNTY}

FOUNTAINE, CHARLES F

DIRECTOR AND MEASURES

RD 2

FORT ANN NY 12827

$518632-5648$

\section{WAYNE COUNTY}

DE NAGEL - RICHARO W

DIRECTOR

DEPT OF CONSUMER AFFAIRS WEM

PO BCेX 369

LVONS. NY 14489

$315946-4512$

\section{WESTCHESTER COUNTY}

HAL E. KENNETH H

OIRECTOO

DEPT CONHSU!MER AFFAIRS \& WEM

CRUNTY OFFICE BLDG ROOM 104

WHITE PLAINS. NY IC6OL

$914285-215$.

SMALEC WILLLAM

INSPECTOQ WEIGHTS 6 MEASURES

WESTCHESTER COUNTY

234 ELM ST

YONKERS. NY 10701
$914963-4541$

WYOMING COUNTY

DANIEL CHARLES A

COUNTY OF WYOMING

COUNYY OF WYOMNG

STRYKERSYILLE. NY 14145

$716535-7816$

YATES COUNTY

COON PAUL $S$

DIRECTOR

3614 BATH ROAD

PENN YAN. NY 14527

315 5.36-8546

CITY: COHOES

PASSARETTI DOMINICK

DIRECTOR

3 GEORGE ST

COHOES: NY 12047

CITY: DUNKIRK

MUSCATOR ROBERT C ${ }^{C}$ MEASURES

DIRECTOR

DUNKIRK! NY 14048

716 366-0777

CITY: FULTON

BOYNTON: FLOYD Ĺ

DIRECTOR OF HE

25 INGER SOLL DR

FULTON NY 13069

315 593-6.636

CITY: HUDSON

EGAN.JJAMES

DIRECTOR

HARRY HOWARD AVE

HUDSON. NY 12534

$518828-1628$ 
YATSIL MARTIN

PEDCNE STEFANO $J$

OFFICE CF CONSUMER PROTECTION

CITY HALL

MT VERNDN NY 10550

$914668-2200 \times 201$

CITY: NEW YORK

CAPERS BASIL H

SUPERVISING INSPECTCR 245 MESEROLE AVE

BROCKLYN NY 11222

$212566-2819$

CARUSD, SAL VATORE

INSPECTOR

DEPT OF CONSUMER AFFAIRS

80 LAFAYETTE STREET

NEW YORK. NY 11372

$212457-6604$

FELD, MELVYN L

SUPERYISING INSPECTOR

N $Y C$ DEPT OF CONSUMER AFFAIRS 41-39 248 ST

212 566-2819 NY 11363

GOLDSTEIN, BURTON

DIRECTOR ENF CONSUMER AFFAIRS

80 LAFAYETTE STREET

$212566-5815$

GREY LLOYO A

PR INCIPAL INSPECTOR

CONSUMER AFFAIRS

BO LAFAYETTE ST

NEW YORK. NY 10013

212 566-8776

KELLEY, ROBERT $B$

SUPERVISING INSPECTOR

22 MAINE AVE

STATEN ISLAND. NY 10314

$212566-4279$

KILEY RCBERT

DIRECTOR OF ENFORCEMENT

DEPT OF CONSUMER AFFAIRS

80 LAFAYETTE ST

NEW YORK NY 10013

212 566-5815

KLEINMAN。 MORRIS

INSPECTRR

OEPARTMENT OF CONSUMER AFFAIRS

80 LAFAYETTE ST
NEW YORK N
212 566-5805
20013

KUTZBACH, DALE

DIRECTOR CONSUMER AFFAIRS

80 LAFAYETTE ST

NEW YORK. NY 10013

$212566-5815$

MBR

NCWM

MBR

NCWM

MBR

NCWM

LEMPIN PETER

ASSISTANT COMMISSIONER

NY CITY DEPT OF CONSUMER AFFRS

80 LAFAYETTE ST

NEW YORK. NY 10013

$212566-5817$
YC DEPT OF CONSUMER AFFAIRS

80 LAFAYETTE ST

NEW YORK NY 10013

MUL BERG J EROME

SR CONSUMERS AFFRS INSPECTOR

NEW YORK CITY DEPT CONS AFFRS

80 LAFAYETTE ST ROCM 320

NEW YORK
$2125 \%$
$566-2247$

PETITO\& JOHN J

SUP ERV I SER INSPECTCR

DEPT OF CONSUMER AFFAIRS

638 TENTH ST

BROOKLYN. NY 11215

ROSENTHAL, STUART

DEPARTMENT OF CONSUMER AFFAIRS 80 LAFAYETTE ST NEW YORK. NY 10013

ROSS: JONATHAN

DIRECTOR ADJUD ICAT ION DIVISICN

NYC DEPT CONSUMER AFFAIRS

80 LAFAYETJE ST

NEW YORK NY 10010

SHERMAN, HAROLD I

PRINCIPAL INSPECTOR

OEPARTMENT OF CONSUMER AFFAIRS

8 O LAFAYET TE ST

NE.W YORK NY 10013

$212566-5805$

STERN, SIDNEY

LABORATORY DIRECTOR

BDARD OF EDUC BUR CF SUPPLIES

44-36 VERNON BLVD

NEW YORK. NY 11101

$212626-2973$

CITY: OSWEGO

ADAMS, ERNEST A

SEALER WEIGHTS AND MEASURES

CITY OF OSWEGO

CITY HALL

DSWEGQ: NY 13126

CITY: PLATT SBURGH

BURNS - LCYAL

DIRECTOR

84 BOYNTON AVE

PLATTSBURGH. NY 12901

$518563-4588$

CITY: PORT JERVIS

GRAY WILL I AM

DIRECTOR

77 BALL ST

PORT JERVISE NY 12771
$914856-8827$

CITY: RENSSELAER

DOWNES . KENNETH

DIRECTOR

WEIGHTS AND MEASURES

3 INDI ANA AVE

RENSSELAER NY 12144

$518286-3972$ 
$Y:$ ROME

HIELKE RAYMOND H \& MEASURES NCRM ITY HALL ON THE MALL $O M E N Y 13440$

$15336-6000 \times 238$

YY: SCHENECTADY

IASSARONI, DANTE A

II RECTOR

3UREAU WEIGHTS E MEASURES

ITY HALL JAY ST

SCHENECTADY, NV 12305

$518382-5061$

TY: TROY

MYERS PATRICK A

DIRECTOR WEIGHTS ANO

519 FOURTH ST

TROY $273-412180$

TV: UTICA

BAZAN ANTHONY R

DIRECTOR

UITY HALL 13502

$315792-0260$

ITY: WATERVLIET

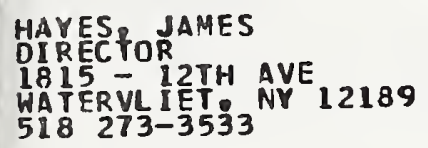

ITV: WHITE PLAINS

STEVENS, EUGENE $C$

DIÜECTOR CF WEIGHTS 6 MEASURES

DEPT OF PUBLIC SAFETY

279 HAMILTON AVE

WHITE PLAINS, NY 10601

$914682-4273^{\circ}$

:ITY: YONKERS

CAPOZZI RALPH A

MBR DIRTS E MEÁS E CONSUMER PROTEC CITY HALL

YONKERS. NY 10701

$914964-3563$

CAROLINA

STATE

AMCS, LEE ROY

STANDARDS DIVISION

NC EEPT OF AGRICULTURE

$P A$ BOX 27647

KALEIGH NC 27611

$919733-3313$

$M B R$

NCWM

MBR

MBR
AYERS SPENCER A

STANDARDS DIVISION

NC DEPT OF AGR ICUL TURE

PO BOX 27647

RALEIGH. NC 27611

BARNES JUAMES G

NCA DIV HEI GHTS $\&$ MEASURES

NC DIV WEIGHTS E

RALEIGH. NC 27603

$919772-3834$

BUTLER JAMES M

STANDARDS OIVISION

NC DEPT OF AGR ICUL TURE

PO BOX 27647

RALEIGH NC 27611

$919733-3313$

CHRISTIE, JOHN W

STANDARDS DIVISION

NC OEPT OF AGR ICUL TURE

PO BOX 27647

RALEIGH NC 27611

$919733-3313$

CLARK. OSBY

STANDAROS DIVISION

MC DEPT OF AGR ICULTURE

PO BOX 27647

RALEIGH: NC 27611

CRAVEN LARRY $C$

STANDARDS DIVISION

NC DEPT OF AGR I CUL TURE

PO BOX 27647

RALEIGH- NC 27611

DIX, HAYNE

STANDARDS DIVISION

NC DEPT OF AGR ICUL TURE

PO BOX 27647

RALEIGH NC 27611

919-733-3313

DUNN. BOBBY

METROLOGIST

DEPT OF AGRICULTURE

STANDAROS DIV/PO BCX 27647

RAIEIGH. NC 27611

$919733-4411$

EASON' METROL IF

STANDARDS DIVISION

RALEIGH. NC 27611

$919733-441$

FOSTER, CUARE: EN L

STAMDAROS DIVTSION

NC DFPT CA AGR ICUL TURE

OO $\mathrm{A}$ X $\times 2764$ ?

PLE

$919733-3313$

GORDON ROBERT L

OIR EUTJD \& IMUG PRCTECT

NC DEPT OF ACR ICUL TURE

PO BOX 27647

RALEIGH: NC 27611

GRIFFIN RONALD E

STANDARDS DIVISION

PO BOX 27647

RALEIGH. NC 27611

$919: 733-3313$
MBR 
HALLE GRADY F

WESTERN AREA SUPERVISOR

4321 CAKOALE ROAD

CHARLOTTE, NC 28216

704 393-3003

HAROIN LESTER B

STANDARDS DIVISION

NC CEPT CF AGRICULTURE

PO BOX 27647

RALEIGH. NC 27611

$919733-3313$

HIGGINS JESSE D

STANDARDS DIVISION

NC DEPT OF AGRICULTURE

P.O BOX 27647

RALEIGH: NC 27611

$919733-3313$

HUNTLEY III, JOSEPH W

STANDARDS DIVISION

NC CEPT OF AGRICULTURE

PO BOX 27647

RALEIGH NC 27611

919-733-3313

LINDSAY CURTIS

STANDAROSS DIVISION

NC DEPT OF AGRICULTURE

PO BOX 27647

RALEIGH: ${ }^{N C} 27611$

MURDCCK RONALD D

101 ANGUS COUR

$919481-1912$

NEL SON JAMES K

STANDARDS DIVISION

NC DEPT OF AGRICULTURE

PO BOX 27647

RALEIGH: NC 27611

PEAKS RANDOLPH F

STANDAROS DIVISION

NC CEPT OF AGRICULTURE

PO BOX 27647

RALEIGH NC 27611

PERRY, DENNIS G

STANDARDS DIVISION

NC DEPT OF AGRICULTURE

PO BOX 27647

RALEIGH NC 27611

$919733-3313$

PERRY DCNNIE G

STANDARDS DIVISION

NC DEPT OF AGRICULTURE

PO EOX 27647

RALEIGH: NC 27611

PL UMMER JASON A

STANDARDS DIVISION

NC DEPT OF AGRICULTURE

PO BOX 27647

RALEIGH NC 27611

SCOTT TOM

CHIEF MEASUREMENT SECTION

MBR

STNDARDS DIV

RALEIGH NC 27611

MER
SMITH. N DAYID

DIRECTOR STANDAROS OY

NE DEPT DF AGR ICULTURE

PO BOX 27647

RALEIGH ${ }_{919} \mathrm{NC}_{33} 27611$

TAYLOR - W DURWARD

EASTERN AREA SUPER VISOR 205 HANOVER ST

WILLIAMSTON: NC 27892

TEACHEY SHERRY

STANDARDS DIVI SION

NC DEPF OF AGRICULTURE

PO Bก̃X 27647

RALEIGH. NC 27611

$919733-841$

TEW, CHERY A

STANDÁRUS DIVISION

NC DEPI OF AG.RICULTURE

PQ BOX 27647

RAL IGH. NE 27611

$919-733-441$

TURNER RIEHARD W

STAADAROS DIVISION

NE DEPT DF AER ICULTURE BO 27647

RA19 7GH- NC 2 T611

VINES, PATRICK N

STANDAROS DIVISION

NC DEPT OF AGR ICUL TURE

PO BDX 27647

RALEIGH. NC 27611

$919733-3313$

NORTH DAKOTA

STATE

BIANCŪ. BILL

INSPECTSR MEASURES DIV

920 N I 3TH ST 58501

BLASY ALLEN

INSPECTOR

WEIGHTS E MEASURES DIV

RT 3 ROX 11 ÉK

DICKINSON VD 58601

JORDHEIM. CAVIO

INSPECTOR

WEISTITS \& MEASURES OIV

THOMPSON. ND 58278

NIEBERGALL BRUCE R

OIRECTOR

STATE CAPITÖL

OISMARCK NO 58505

NIESE WALT

WEI GHTS \& MEASURES CIV NEW SALEM, ND $58563^{\circ}$

RICHTER: PHYLL IS

INSPECTOR

WEIGHTS \& MEASURES DIV 436 8TH ST NH

VALLEY CITY. ND 58072 
AAAEEN JIM

NSPECTOR

WEIGHTS \& MEASURES DIV

OT 22/HANSON TRAILER COURT

IALLEY CITY. ND 58072

ATE

BADENHOP ROBERT

TIV OF WEIGHTS \& MEASURES

8995 E MAIN ST BLOG \#5
REYNOLDSBURG OH 43068

614 866-6361

DE SALVO BARBARA

SUPERVISOR

DIV WEIGHTS \& MEASURES

8995 E MAIN ST BLDG 75

REYNOLO SBURG. OH 43068

$614866-6361$

DOWNEY DON

INSPECTOR

8995 E MAIN ST BLDG \#5

REYNOLD SBURG OH 43068

$614866-6361$

GRAY, JCHN R

SUP ERYI SOR

DIV OF WEIGHTS \& MEASURES

8995 E MAIN ST BLDG

REYNOLDSBURG OH 43068

614 866-6361

LAWSONE ALVIN F

DIV OF WEIGHTS \& MEASURES

8995 E MAIN ST BLOG 5

REYNOLD SBURG OH 43068

$614866-6361$

\section{LAWSON ROGER \\ INSPECTCR \\ OIV OF WEIGHTS \& MEASURES \\ 8995 E MAIN ST BLOG \#5 \\ REYNOLOSBURG. OH 43068 \\ $614866-6361$}

\section{LITZENBERG BRUCE}

DEPUTY DIRECTOR E CHIEF

DIVISION OF WEIGHTS 6 MEASURES

Q995 E MAIN ST

REYNOLDSBURG O OH 43068

614 866-6361

MALLOW GREG

INSPEC OR

DIV OF WEIGHTS \& MEASURES

8995 E MAIN ST BLDG \#5

REYNOLDSBURG OH 43068

$614866-6361$

MAURER STEVEN D

DIRECTOR

OHIO DEPT OF AGRIC

65 S FRONT ST

CDLUABUS 3 OH 43215

O CCNNOR, JOHN P

DIV OF WEIGHTS \& MEASURES

8995 E MAIN ST BLDG

REYNOLOSBURG OH 43068

614 866-6361
RINE: LARRY

DEPARTMENT OF AGRICULTURE

8995 E MAIN ST

REYNOLOSBURY. OH 63068

$614866-6361$

SMITHE STEVE

INSPECTOR

8995 E MAIN ST BLDG H5

REYNOLDSBURG. OH 43068

$614866-6361$

TRUEX JAMES C

WEIGHTS $\&$ MEASURES INSP MGR

OHIO DEPT OF AGRI CULTURE

8995 E MAIN ST

REYNOLOSBURG. OH 43068

$614866-6361$

HEST' HILLIAM

DIV OF WEIGHTS $\&$ MEASURES

8995 E MAIN ST BLDG HTS

REYNOLDSBURG OH 43068

$614866-6361$

WHEELER KENNETH

INSPECTOR E TRAINING OFFICER

DIV OF HEIGHTS \& MEASURES

8995 E MAIN ST BLDG W5

REYNOLDSBURG. OH \&3068

614 866-6361

ADAMS COUNTY

ROBERTSO RICHARD

INSPECTOR WEIGHTS E MEASURES

ADAMS COUNTY

770 DANIEL RD

WINCHESTER OH 45697

$513544-2364$

STONE, WILLIAM J

COUNTY AUDITOR

ADAMS COUNTY

COURTHOUSE

HEST UNION, OH 45653

513 544-2364

ALLEN COUNTY

CASHELL CHUCK

INSPECTOR WEIGHTS $\triangle$ MEASURES

ALLEN COUNTY

850 RUNYAN ST

LIMA. OH 45801

$419228-3700 \times 232$

FRENCH - H DEAN

COUNTY AUDITOR

ALLEN COUNTY

COURTHOUSE

LIMA. OH 45801

$419228-3700 \times 236$

ASHLAND COUNTY

CLAYTONE THOMAS

INSPECTOR WEIGHTS $\&$ MEASURES

ASHLANO COUNTY

COURTHOUSE

ASHLAND. OH 44805

$419289-0930$

RYLAND. S E

COUNTY AUDITOR

ÁSHLANO COUNTY

COURTHOUSE

ASHLAND. OH 44805

$419289-0000$
MBR

NCWM 
DIANGELO, JEFF

INSPECTOR WELGHTS \& MEASURES

ASHTABULA COUNTY

25 J JEFFER SON ST

JEFFERSCN 216 DH 44047

ROSS D DAIEL P

ASHTABULA COUNTY

COURTHDUSE

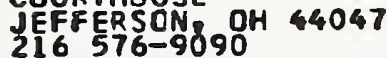

ATHENS COUNTY

COULADIS PETER G

COUNTY AUDIYTR

COURTHOUSE

ATHENS OH 45701

$614593-3166$

MINGUSP ROBERT J

AUDITOR OFFICE

3 N SHANHON AVE

ATHENS. OH 45701

614 593-6794

AUGLAIZE COUNTY

KING RCBERT E

INSPECTOR WEIGHTS E MEASURES

MBR

209 E PEARL ST

HAPAKONETA OH 45895

$419738-2364$

SCHUMAN KARYN

COUNTY AUDITOR

AUGLAIZE C

HAPAKONETA, OH 45895

$419738-2511$

\section{BELMONT CCUNTY}

PAPPANO JOSEPH A

COUNTY AUDITOR

COURT HOUSE

ST CLAIRSVILLE, OH 43950
$614695-2121$

THCRNTON HOWARD

INSPECTOR WEIGHTS E MEASURES BELMONT COUNTY

$30 \mathrm{~N}$ CHESTNAS

BARNESVILLE, OH 43713

$614695-2121 \times 236$

\section{BROWN COUNTY}

ERNSTY HAROLDE

BRCWN CCUNTY

COURTHOUSE

$513{ }^{3} 378-63988^{O H} 45121$

GASKIN: MILLARD

INSPECTCR

BROWN COUNTY

COURTHOUSE

GEORGETCWN, OH 45121
HAWKINSS CARL

130 HIGH ST

HAMILTON OH 45011

$513867-5796$

MARSH, ALAN V

SUPERVISOR WEIGHTS \& MEASURES

130 HIGH ST

HAMILTON OH 45011

PHALEN CLARENCE A

ASSISTANT COUNTY AUDITOR

130 HIGH ST

HAMILTON OH 45011

$513867-5796$

RICE, JOHNEE

BUTLER COUNTY

130 HIGH ST

HAMILTON, OH 45011

$513867-5794$

TILTON RAY

INSPECTOR

130 HIGH ST

HAMILTON
$513867-5794$

WURST: DANIEL S

AUDITOR

BUTLER CDUNTY

HAMILTON OH 45011

$513867-5793$

WURST: DANIEL $S$

AUDITOR

BUTLER CDUNTY

130 HIGH STREET

HAMILTON CH 45011

$513867-5756$

ZECHER KR I STOPHER

INSPECTOR

BUTLER CDUNTY

130 HIGH ST

HAMILTON OH 45011

$513867-5793$

CARROLL COUNTY

GUESS. MAC

COUNTY AUDITOR

CARROLL COUNTY

COURTHOUSE

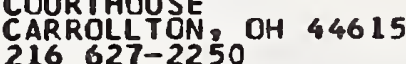

KINCH. IRV

COUNTY AUOITOR SEALER

CARRDLL CDUNTY

COURTHOUSE

CARROLLTON NOH 44615

$216627-2250$

CHAMPAIGN COUNTY

CRUMLEY̊NELLIE

INSPECTOR

CHAMPAIGN COUNTY

COURTHOUSE 43078

URBANA: OH 43078 
ARMAN. BONNIE M OUNTY AUDITOR HAMPAIGN COUNTY OHIC

MAIN E W COURT

$13653-7775$

IRK COUNTY

PECK ROY $K$

NSPECTCP WEIGHTS GE MEASURES

¿ARK COUNTY AUDITORS CFFICE

B GRAHAM BLOG POB 1325

$313328-2424$

SHUTTLEWCRTH JERRY

-OUNTY AUDITOR

¿LARK CCUNT

COUNTY BUILDING

SPR INGF IELD OH 45502

$513328-2424$

\section{ERMCNT COUNTY}

KOCH, FRED H

INSPECTCR

CLERMONT CDUNTY

270 MAIN ST

BATAVIA. OH 45103

$513732-7151$

RYMER, JAMES R

COUNTY AUDITOR

CLERMONT CE

BATAYIA. OH 45103

$513732-7226$

RYMER , JAMES $R$

COUNTY AUDITOR

CLERMONT COUNTY

BATAVIA. DH 45103

$513732-7226$

LINTON COUNTY

ARMSTRONG, WANDA

COUNTY AUDITOR

CLINTGN COUNTY

COURTHOUSE

WILMINGTCN, CH 45177

$513382-225$

RICHARDSON CHARLES A

INSPECTOR WEIGHTS \& MEASURES

CLINTON COUNTY

627 KATYS LANE

WILMING TCN. OH 45177

$513382-2816$

DLUMEI ANA COUNTY

BELL, KENT

CDUNTY AUDITOR

COLUMBI ANA COUNTY

COURTHOUSE

LISBON OH 44432

$216424-9511$

GERACE JIM

COLUMBI ANA COUNTY

COURT HOUSE AUDITOR OFFICE

LISBON. OH 44432

$216424-9511$
MBR
NCWM

COSHOCTON COUNTY

BESST. RQGEP

INSPECTOR WEIGHTS \& MEASURES

COSHOCTON COUNTY

45844 CR 23

COSHOC. TON OH 43812

$614622-1243$

MBR

NCWM

TOMPKINS, RICHARD J

COUNTY AUDITOR

COSHOCTON COUNTY

COURTHOUSE ANNEX BLDG

COSHOCTON. OH 43812

$614622-1243$

CRAWFORD COUNTY

HILDENBRAND AL

INSPECTOR WEIGH

CRAWFORD COUNTY

BUCYRUS DH 44820

MBR

NCWM

LONG, DONALD E

AUDITOR

CRAWFORD COUNTY OHIO

$P$ O BOX 150

BUCYRUS. OH 44820

$419562-7941$

CUYAHOGA COUNTY

BOVA. FRANK

DIRECTOR WEIGHTS \& MEASURES

CUYAHOGA COUNTY

1219 ONTAKIO ST ROCA 33

CLEVELAND. OH 44113

$216443-70,55$

DAVISQTEMOTHY

CUY AHIOGA CELUVTY

1219 ONTARIO ST ROCM 33

$216843-7035$

HEADEN, ORIAN

INSPECTOR WEICHTS \& MEASURES CUYAHOGA COUNTY

1219 ONTARIO ST ROCN 33

CLEVELAND。 DH 44113

$216443-7035$

MCCORMACK, J TIMOTHY

COUNTY AUDITOR

CUYAHOGA COUNTY

I?I9 ONTAR IO' ST

IEVELAND. OH 44113

$216443-7035$

HCNEFF, BILL.

INJPECTOR GEIGHTS \& MEASURES

MBR

CUYAHDGA COUNTY

CLEVELANO OH 44113

216 4 $3-7035$

NEWSOMF, RDBERT

CHIEF INSPECTOR

FUY AHOGA COUVTY

(219 UNTARIO ST ROOH 33

CLEVELAND. OHH 44113

$216443-7035$

RAGER JOS CPH

INSPECTOR AFIGHTS $E$ MEASURES

CUYAHOGA CDUNTY

1219 ONTARIO ST ROCM 33

CLEVELAN.OO OH $\$ 4113$

2 is $443-703$ :
MBR

NCWM 
WISEO HGWARD

INSPECTCR WEIGHTS \& MEASURES

CUYAHOGA COUNTY

1219 ONTARIO ST RCOM 33

CLEVELAND $216453-7035$

DARKE COUNTY

BAKER, HERMAN M

INSPECTOR WEIGHTS \& MEASURES

DARKE COUNTY

3114 GORDON LANDIS RO

ARCANUM OH 45304

$513692-5235$

RNICKE NORMA FAE

COUNTY AUDETOR

DARKE CCUNTY

DARKE CE COURTHOUSE

GREENVILLE OH 45331

$513548-2235$

DEFIANCE CCUNTY

COLEMAN YERNA A

COUNTY AUDITOR

DEF I ANCE COUNTY

COURTHOUSE

DEFIANCE OH 43512

DELAWARE COUNTY

RICHARDS, JOHN C

INSPECTOR

OELAWARE COUNTY

523 W WILLIAMS

DELAWARE OH 43015

$614363-1736$

THOMAS. DAVID R

COUNTY AUDITOR

DELAWARE COUNTY

COURTHOUSE

DELAWARE OH 43015

$61 \% 369-8761$

\section{ERIE COUNTY}

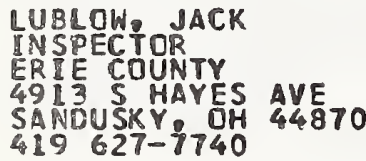

MCKEEN JAMES $W$

COUNTY AUDI TO

COURTHOUSE

SANDUSKY OH 44870

$419627-7740$

FARRFIËLD COUNTY

AL $2 E N, 3 I M$

IN SPECTOR

FAIRF IELD COUNTY

633 SMITHF IELD

$\angle A N C A S T E R$ OH 43130

$614687-7160$

REIC J JAMES P

FAIRFIELD COUNTY

COURTHOUSE

LANCASTER OH 43130

$614687-7020 \times 234$
FAYETTE COUNTY

JOSEPH. HARY LDU

CDUNTY AUD I TOK

FAYETTE COUHYY

C.JUR YHDSE

MASH 335 - 4461 , OH 43160

RANKINE MARLENE
INSPECTOS WEIGHTS \& MEASURES

FAYETYE COUNTY

3607 SP. मू 4 I NII

WASHINGTON C.H. CH 43160

$614335-0858$

FRANKLIN COUNTY

BOWMANE SCOT:

INSPEC.TOR

FRANKLIN $\mathrm{CO} \otimes \mathrm{E}$

410 S H IGH $5 \mathrm{~T}$

COLUMBUS OH 43215

$614462-3742$

GIAMMARCC. ELSA

FPANKL IN COUNTY W E $M$

410 S HI GH ST

COLUMBUA, OH 43215

$614462-3742$

JONES: CAVIO W

INSPECTCR HEIGHTS \& MEASURES OFFICE OF WEIGHTS \& MEASURES $4105 \mathrm{HIGH}$ 5T

COLUMBUS CH 43215

$614462-3742$

HCCAPTY WILLIAM G

OFFICE OF WEIGHTS \& MEASURES

410 S HIGH ST

COLUHBUS $462-3742$

MCNEAL. PALMER

AUD ITOR

FRANKLIN COUNTY

CCLUMBUS. OH 43215

$614 \quad 462-3223$

PETRELLA: MIKE

FRANKLIN CO HEIGHTS \& MEASURES

$410 \mathrm{~S}$ HIGH ST

C.CLUMBUS. OH 43215

$614462-3742$

FUL TOH COUNTY

GOIVERS LELAND

IPSPECTOR HEIGHTS $E$ MEASURES

210 FULTCN ST

WALS SECN! OH 43567

$419335 \div 69: 1$

TEUDEL. SOHN L

CCUNTY AUDITCR

FULTON COUNTY

210 S FUETON ST

WAU SEON OH 43567

$419335-6911$

MBR

GALLIA COUNTY

CANADAY, RONALD

COUNTY AUDITOR

GALLIA COUNTY

COURTHOUSE

GALLIPOLIS $\mathrm{OH} 45631$ 
IOUCK JAPETES E HERTS E MEASURES ALLIA COUNTY OURTHOUSE

ALLIPOLIS OH 45631

$14466-4612$

IUGA COUNTY

IAKCWSKI RICHARD J

:OUNTY AUDITOR

GEAUGA COUNTY

219 MA:N ST ANNEX

HAROON. OH 44024

216 285-2222

MALCUIT ARTHUR

INSPECTOR WEIGHTS \& MEASURES GEAUGA COUNTY

219 MAIN ST

CHARDON OH 44024

$216285-2222$

\section{EENE COUNTY}

HOLLING SWORTH, NEIL J INSPECTCR WEIGHTS \& MEASURES GREENE COUNTY

69 GREENE ST ROCM 200

XENIA OH 45385

$513376-5066$

SHAW DOROTHY L

COUNTY AUD ITOR

GREENE COUNTY

COURTHOUSE-69 GREENE ST

$X E N 1 A, C H 45385$

$513376-5065$

JERNSEY COUNTY

PATTERSON CHRIS

INSPECTCR WEIGHT

RT 13 1 1141 CADII RO

CAMBRIDGE OH 43725

$614432-5296$

PATTERSON LEONARD

COUNTY AUDITOR

GUERNSE Y COUNTY

COURTHŌSE

CAMBRIDGE OH 43725

IAMILTON COUNTY

DECCURCY JR JOSEPH L

CEUNTY AUOI I TOR

HAMILTON COUNTY

1000 MA IN ST

CINCINNATI OH 45224

$513632-8212$

FI TZPATRICK • GREG

INSPECTOE

HAMILTON COUNTY

6477 MONTGOMERY RO

CINCINNATI OH 45213

513 $21-2814$

GERTLER RAY

HARILTON COUNTY

5414 TLOURY COURT

CINCINNATI OH 45238

$513821-2814$
PUCKETT THOMAS

SUPERVISOR-WEIGHTS \& HEASURES HAMILTON COUNTY AUDITOR

6505 VINE ST

CINCINNATI OH 45216

HANCOCK COUNTY

DAYIS. ORLAF

NSPECTOR WEIGHTS \& MEASURES

HANCOCK COUNTY AUD ITOR

20 CENTRAL AVE

INDLAY. OH 45840

$419422-1351$

HUGUS. EDWARD $K$

COUNTY AUDITOR

HANCOCK COUNTY

COURTHOUSE 330 S MAIN

FINDLAY. OH 45840

$419424-7015$

HAROIN COUNTY

WARREN. JOHN T

INSPECTOR WEIGHTS $\&$ MEASURES

HARDIN COUNTY

$8735 \mathrm{CO} R \mathrm{OD} 200$

BELLE CENTER. OH $\$ 3311$

$419673-5232$

HOODS. MARILYN

COUNTY AUDITOR

HARDIN COUNTY

COURTHOUSE

KENTON: OH 43326

$419673-5232$

HARRISON COUNTY

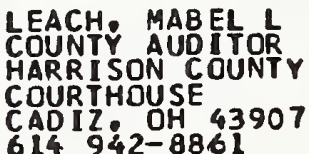

TAYLOR SAMUEL E HARR ISON COUNTY

COURTHOUSE $100 \mathrm{~W}$ MARKET ST

CAD IZ. OH 43907

$614942-8861$

HENRY COUNTY

CLAYTON. MAX W

INSPECTOR

WEIGHTS AND MEASURES

$3.31 N$ PARK ST

DESHLER:DH 43516

HOOPS. JAMES

COUNTY AUD ITOR

HENRY COUNTY

COURTHOUSE

NAPOLEON OH 43545

419592-1956

HIGHL AND COUNTY

DAVISE RALPH

INSPECTOR HEIGHTS $\&$ MEASURES

HIGHLAND CGUNTY

7421 WOODMANSEE RD

LEESBURG OH 45135

513 780-7535 
TEETERS LEWIS B

COUNTY. AUDITOR

P GHLAND COUN

HILLSBOROP DH 45133

$513393-1915$

HOCK ING COUNTY

HUNTER JEFF S

HOCKING COUNTY

AUDITORS OFFICE COURTHOUSE

LOGAN OH 43138

MYERS, LEONARD A

COUNTY AUDITOR

COUNTY COURTHDUSE

LOGAN, OH 43138

$614385-2127$

HOLMES COUNTY

PYLE KEVIN

COUNTY AUDITOR

HOLMES COUNTY

COURTHOUSE

MILLERS BURG OH 44654

WEAVER JOHN R

INSPECTOR WEIGHTS \& MEASURES

MBR

HOLMES COUNTY

PO BOX 65/5425 TR 358

BERLIN OH 44610

$216893-2255$

HURON COUNTY

CUNNINGHAM, RICHARD D

DEPUTY SEALER

HURCN CCUNTY
36 STOUTENBURY DR

NORWALK. OH 44857

$419668-8643$

ELMLINGER, JOHN

COUNTY AUDITTR

HUPCN COUNTY COURTHCUSE

NOR

$419668-4304$

\section{JACKSCN COUNTY}

CLARK, DONALO

COUNTY AUDITOR

COURTHOUSEYMAIN ST

JACKSON. OH 45640

$614286-4231 \times 244$

WINTERS RODNEY

INSPECTOR WEIGHTS \& MEASURES

JACKSON COUNTY

WELLSTOR, OH 45690

$614286-4231$

\section{JEFFERSON COUNTY}

CANESTRARD, RICHARD W

COUNTY AUDITOR

JEFFERSON COUNTY

P C BOX 159

STEUEENVILLE $\mathrm{OH} 43952$

MBR
ZORBINI VINCENT A

INSPECTOR WEIGHTS E MEASURES JEFFERSON COUNTY

301 CARL ISLE AVENUE

MINGO JUNCTION. OH 43938

$614283-1944$

\section{KNOX COUNTY}

DAUBENSPECK L LARRY E

INSPECTOR WEIGHTS $\&$ MEASURES KNOX COUNTY

$6221 / 2$ MAIN

MOUNT VERNON,OH 43050

$614397-2727$

MODDY. WILLIAM

COUNTY AUDITOR

KNOX COUNTY

106 E HIGH ST

MT VERNON OH 43050

$614397-2727$

\section{LAKE COUNTY}

MORTON, HOHARO G

INSPECTOR WEIGHTS 6 MEASURES

LAKE COUNTY

112 MURPHY ST

GRAND RIVER: OH 44045

$216357-2534$

SHOOK, TERRANCE

INSPECTOR WEIGHTS $\&$ MEASURES

305 CHESTNUT ST

PAINESVILLE. OH 44077

$216352-1701$

NCWM

ZUPANCIC, EDWARD J

COUNTY AUDITOR

LOSE MAIN ST BOX $49 \mathrm{C}$

PAINESVILLE, OH $44 \mathrm{C} 77$

$216357-2534$

\section{LAWRENCE COUNTY}

JENKINS, G L

INSPECTOR WEIGHTS 6 MEASURES

LAWRENCE COUNTY

1057 MAR IDN PIKE

IRONTON. OH 45638

$614533-4310$

PAYNE. JAMES A

COUNTY AUDITOR

LAWRENCE COUNTY

COURTHOUSE-VETERAN SO

IRONTON. OH 45638

$614533-4310$

\section{LICKING COUNTY}

BUCHANAN, GERRGE D

COUNTY AUD ITOR

LICKING COUNTY

20 S 2ND ST CO BLDG

NEWARK. $\mathrm{CH} 430$

WILSON JR, EARL H

INSPECTOR WEIGHSS \& MEASURES ICKING COUNTY AUOITOR S CFF ICE $79 \mathrm{~N} 21$ ST

NEWARK. OH 43055

$614349-8421$ 
ONES, JEAN

OUNTY AUDITOR

OGAN COUNTY

EILFONTANE, OH 43311

II $1399-7209$

IEWFAN CHARLES E

NSPECTOR

OOUNTY WEIGHTS E MEASURES

OO S MADRIVER ST

3ELLEFOATAINE, OH 43311

;13-592-6701

RAIN COUNTY

\section{;OLDBERG DAVIDM LORAIN COUNTY BLG 226 MIDDLE AVE ELYPIA, OH 44035 \\ $216329-5207$ \\ PI JCR, JUL IAN A \\ LORAIN COUNTY AUDITOR \\ LORAIN CCUNTY \\ COURTHOUSE \\ EL YRIA: OH 44035}

INSPECTOR WEIGHTS \& MEASURES

\section{CAS CCUNTY}

LEWANDOWSKI D DAVID

CGUNTY AUDITOR

LUCAS CCUNTY

ERIE \& ADAMS ST

$419259-8690^{\circ}$

LUTTMAN, CHRI STA

DIRECTOR WEIGHTS \& MEASURES

LUCAS CCUNTY

GOVT CTR/SUITE 600

TOLEDO, OH 43604

$419245-4358$

\section{DISCN COUNTY}

SANFORD, JOHN L

INSPECTOR WEIGHTS \& MEASURES

MADISON COUNTY

470 JOSIAH MORRIS RD NE

LONOCN OH 43140

$614852-9717$

WEIMER RAYMONO $M$

MADISON COUNTY

COURT HOUSE

LCNDCN: CH 43140

AHONING CUUNTY

RIMEDIO DOMINIC A

INSPECTOR HEIGHTS \& MEASURES

MAHONING COUNTY

YOUNGSTOWN OH 44510

$216747-2000^{\circ} \times 232$

TABLACK, GEORGE J

COUNTY AUDI TOR

MAHONING COUNTY

COURTHOUSE

YOUNGSTOWN $\mathrm{OH}_{24} 4510$
MAHONEY WAYNE

INSPECTOR WEIGHTS 6 MEASURES

MARION COUNTY

COURTHOUSE RODM \#5

MARION
$614387-587133025$

WEBER. LOIS F

COUNTY AUDITOR

MAR ION COUNTY

COURTHOUSE - ROOM 5

MARION: CH 43302

$614387-5871 \times 225$

MEDINA COUNTY

MBR

NCWM

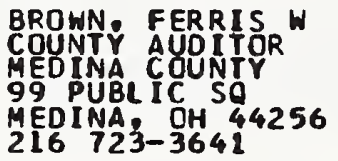

KUHAR , MARK

WEIGHTS AND MEASURES OFFICIAL MEDINA COUNTY 99 PUBLIC SQ

MEDINA. OH 44256

$216723-3641$

MEIGS COUNTY

HICKLINE WILLIAM
COUNTY AUDITOR
MEIGS COUNTY
COURTHOUSE
POMEROY DOH 45769
$614992-2698$

WOLFE HILTON

INSPECTOR WEIGHTS 8 MEASURES MEIGS COUNTY

BOX 14

RACINE, OH 45771

614 992-2917

\section{MERCER COLNTY}

GROSS. EUGENE $W$

INSPECTOR HEIGHTS 6 MEASURES

MERCER COUNTY

COURTHOUSE SQUARE

CELINA. OH 45822

419 586-6402

SCHWIETERMAN, ROGER A

DEPUTY AUDITOR

MERCER COUNTY AUDITCR

COURTHOUSE SQUARE

CELINA: OH 45822

$419586-6402$

MIARI COUNTY

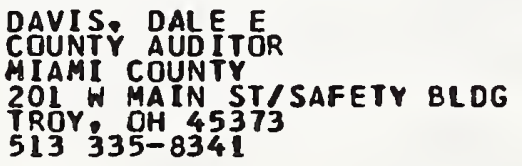

WEAVER, JAMES R

INSPECTOR WEIGHTS 6 MEASURES

MIAMI COUNTY

505 GLENHOOD DR

PIQUA. OH 45356

$513335-8341$
MBR

NCWM 
MOWARD: CARROLL 8

HEIGHTS \& MEASURES INSPECTOR MORROH COUNTY AUDITCR

BRIGGS FREDERICK L MEASURES

MONRCE COUNTY

126 MAPLE AVE 13793

3132 CHESTER TWP RCAD 190

FREDERICKTOWN

$614472-1494$

NEUHART JAMES

COUNTY AUDITOR

MONROE COUNTY

HOOOSFIELD OH 43793

$614472-0873$

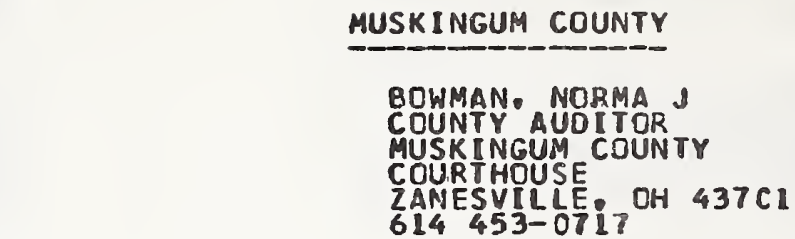

MONTGCMERY COUNTY

HUFFORD DONALD B

MNSPECTOR

S5I H THIRD ST

DAYTCN OH 45422

$513225-4314$

MBR

NCWM

OMLCR, ROBERT $W$

WEIGHTS \& MEASURES MCNTGOMERY 451 THIRD 5

DAYTON OH 45422

$513225-4314$

ROSEBERRY, ROBERT

INSPECTOR WEIGHTS \& MEASURES

MONTGOMERY COUNTY

451 H THIRD ST BOX 972

DAYTON OH 45422

$513225-4315$

STAMPS, DANA A

COUNTY AUDITCR

MONTGOMER COUNTY WGHTS $\&$ MEAS

451 WEST THIRD ST

DAYTON OH 45422

$513225-4333$

MORGAN COUNTY

DUNCANE GLENN

INSPECTOR HEIGHTS \& MEASURES

MORGAN COUNT

RT 킨 $B O X 71$

MCCENNELSVILLE: OH 43756

WOOCWARD. GARY

AUD ITOR

MORGAN CCUNTY

19 E MAIN ST

MCCCNNELSVILLE, OH 43756

$6149.62-447$

\section{MORRCh COUNTY}

HOLTREY, MARY

COUNTY AUDITOR

MORRCW COUNTY

E HIGH ST/COURTHOUSE

MT GILEAD, OH 43.3.9

$415940-4060$

HOWARD, CARRCLL

INSPECTOR HEIGHTS \& MEASURES

MORRCW COUNTY

3132 TWP RD 190

FRECERICKSTGWN. OH $4301 \mathrm{~s}$

$419768-2329$

PAULOING. OH 45879
MBR

MBR

NCWM

\section{PAULDING CCUNTY}

LOTHAMER, BISYNE F

COUNTY AUD ITGR

PAULEING COUNTY

COUKTHGUSE

$419399-4897$

YOH. GEORGE

INSPECTOR WEIGMTS \& MEASURES

PAULDING COUNTY

ROUTE 1

กKWOCD, $\mathrm{OH} 45873$

$-49399-4897$

PERRY CCUNTY

HANYTINSTNO JOAPIN

COIJNTY AUJO ITOR

PERRY CCUN?Y

COURTHOUSEIPO BOX 127

NEW LEXINGTON. CH 43764

$614342-2074$ 
ATTERSON. HARVEY L

NSPECTOR

IE IGHTS MEASURES
6281 LOCUST GRCVE RD

ITLANTA. OH 43104

114 986-8259

ENY MARGARET

OUNTY AUDITÓR

IICKAHAY COUNTY

IRCLEVILLE, OH 43113

iI $14474-4765$

KE COUNTY

3ARKER PAUL T

OOUNTY AUDITC

3 IKE CDUNTY

AAVERLY OH 45690

$514947-2713$

3EEKMAN GARY

INSPECTOR WEIGHTS \& MEASURES

PIKE CDUNTY

6766 GEAVERS RIDGE RD

PEEBLES: OH 45660

$614947-2713$

RTAGE CCUNTY

BI ASELLA, VICTOR

COUNTY AỦDITOR

PORTAGE COUNTY

449 S MER IDIAN ST

RAVENNA. OH 44266

$216297-3561 \times 144$

\section{TURNER, ROBIN}

INSPEC TOR WEIGHTS \& MEASURES

PORTAGE COUNTY

449 S MERIDIAN ST

$216297-3561$

\section{EBLE COUNTY}

MONG BETTY

COUATY AUDITOR

PREBLE COUNTY

100 E MAIN ST/COURT HOUSE

EATCN CH 45320

MOWERY RICHARDE

INSPECTOR WEIGHTS \& MEASURES

PREELE COUNTY

100 E MAIN ST COURT HOUSE

EATCN $\mathrm{CH} 45320$

$513456-8148$

\section{ITNAM COUNTY}

MCDOWELL WILLIAM C

COUNTY INSP WEIGHTS GMEASURES

PUTNAM COUNTY

COURTHOUSE

OTTAWA. OH 45875

419 523-6686

VERHOFF RCSELIA C

COUNTY AUDITOR

MBR

PUTNAM COUNTY

CDURTHOUSE

OTTAWA, OH 45875

$419523-6686$

MBR

NC'WM
RICHL AND COUNTY

GOLDEN. GERALD

INSPECTOR WEIGHTS 6 MEASURES

RICHLAND COUNTY

COURTHOUSE

MANSFIELD OH 44902
$419755-5509$

SHANK. FREEMAN J

COUNTY AUD ITOR

50 PARK AVE E

WANSFIELD. OH 44902

$419755-5501$

\section{ROSS COUNTY}

IOHNSON LLOYD

ROSS COUNTY

153 UNIVER SITY OR/APT 217

CHILLICCTHE: OH 45601

NEAL STEPHEN A

COUNTY AUOITOR

ROSS COUNTY

COURTHDUSE

CHILLICDTHE, OH 45601

SANDUSKY COUNTY

FARRELL H HLLIAM L

COUNTY AUD ITOR

$100 \mathrm{~N}$ PARK AVE/COURTHOUSE

FREMONT: OH 43420

KNISELY, THOHAS B

INSPECTOR WEIGHTS \& MEASURES SANDUSKY COUNTY

1701 CROGHAN ST

FREMONT. OH 43420

\section{SCIOTO COUNTY}

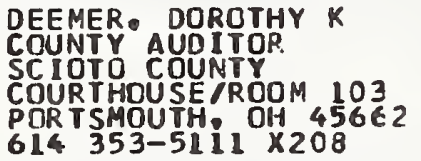

WRI GHT - THOMAS D

INSPECTOR WEIGHTS $\&$ MEASURES SCIOTO COUNTY

RT 2 BOX 241

LUCASVILLE. OH 45648

$614343-5111 \times 232$

\section{SENECA COUNTY}

MILLER, DON

INSPECTOR HEIGHTS \& MEASURES

SENECA CDUNTY

41 N NEH HAVEN ST

BLDONVILLE OH 44818

$419447-0692$

SMITH. RICK

COUNTY AUDITOR

SENECA COUNTY

COURTHOUSE

TIFFIN. OH 44883

$419447-0692$
MBR

MBR

NC WM

NCWM 
HOEHNE, FRANK L

COUNTY AUDITOR

SHELBY COUNTY

COURTHOUSE

SIDNEY, OH 45365

WEHRMAN OONALD F

INSPECTCR HEIGHTS \& MEASURES

SHELBY COUNTY

4380 S R 705

513 LORAMIE 5931 OH 45845

STARK COUNTY

BOWMAN. WILLIAM B

COUNTY AUDI TOR

STARK COUNTY

COURTHOUSE

CANTON, OH 44702

$216438-0356$

KERR。 DEAN $G$

INSPECTOR WEIGHTS \& MEASURES STARK COUNTY

1455 PLEASANT RIDGE SE

MINERVA: OH 44057

$216438-0356$

SUMMIT COUNTY

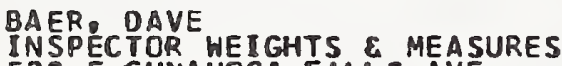

522 E CUYAHOGA FALLS AVE

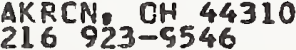

DAVIS. TIM

COUNTY AUDITOR

SUHMIT COUNTY

175 S MAIN ST

AKRON, OH 44308

$216379-2630$

DECHECO. THOMAS O

SUMMIT COUNTY WTS \& MEASURES 522 E CUYHOGA FALLS AV

$A K R C N, O H 44310$
$216923-9546$

DIESZ CHARLES H

SUP ER VI SOR

SUMMIT COUNTY WEIGHTS \& MEAS

522 E CUYHOSA FALLS AVE

$216923-9546$

FROST JAMES

INSPECTOR WEIGHTS \& MEASURES

SUMMIT COUNTY

522 CUYAHOGA FALLS AVE

AKRCN OH 44310

SMIIHY STAN

SUMMIT COUNTY

522 E CUYAHOGA FALLS AVE

AKRON
$216923-9546$

TRUMBULL COUNTY

BUSHY ED

TRUABUL COUNT

$160 \mathrm{~N}$ HIGH NW

HARREN 2 OH 44481
$21684-C 420$

TULL IO. RALPH F

INSPECTOR HEIGHTS \& MEASURES

TRUMBULL COUNTY

$160 \mathrm{~N}$ HIGH NW/ADMIN BLDG

WARREN: OH 44481

$216841-0420$

TUSCARAWAS COUNTY

BEITZEL JOHN A

COUNTY AUD ITOR

TUSCARAWAS CDUNTY

COURTHOUSE/BOX 551

NEW PHIL: OH 44663

$216 \quad 364-8811$

BOTDORF, ERIC

INSPECTOR WEIGHTS 6 MEASURES TUSCARAWAS COUNTY

210 E $20 \mathrm{TH}$ ST

$216364-8811 \times 220$

UNION COUNTY

MBR

NCWM

MBR

NCWM

\section{VINTON COUNTY}

MORGAN, DEBBIE

INSPECTOR WEIGHTS $\&$ MEASURES

VINTON COUNTY

BOX 9/RT I/MORGAN AO

MCARTHUR OH 45651

$614596-5865$

MORGAN - JEAN

COUNTY AUDITDR

VINTON COUNTY

COURTHOU SE

MCARTHUR OH $4565 \mathrm{~L}$

WARREN COUNTY

MINGE, PAUL $V$

INSPECTOR WEIGHTS \& MEASURES

HARREN COUNTY

320 E SILVER ST

$513932-4040$ 
ELSON. NICK OUNTY AUDITOR

ARREN COUNT

OURTHOUSE

EBANON DH 45036

$13932-4040$

MINGTON COUNTY

IARR JAMES

NSPECTOR HEIGHTS \& MEASURES

IA SHING TCN COUNTY OHIO

28 i/2 5 TH ST

IARIETTA: OH 45750

14 373-6623

\section{IERRITT WINNIE \\ OUUNTY AUD ITOR \\ OURTHOUSE \\ AARIETTA, OH 45750 \\ 314 373-6623 \\ INE COUNTY}

FOUCHE - SUE ANN

COUNTY AUD ITOR

$428 W$ L IBERTY ST

WOOSTER = OH 4

MBR

ROBERTSCN, JOHN A

INSPECTOR

WEIGHTS \& MEASURES

86 EVER GREEN LANE

APPLE CREEK OH 44606

216 263-3138

\section{LLIAMS COUNTY}

BERNATH. JAMES T

INSPECTOR WEIGHTS \& MEASURES

WILLIAMS COUNTY

101 VINE/PO BOX 432

STRYKER OH 43557
$419682-4761 \times 2191$

LOUGHEED EVELYN

COUNTY AUOITOR

WILLIAMS COUNTY

COURTHOUSE

BRYAN, OH 43506

$419636-5639$

\section{JO0 COUNTY}

BATESON HAROLD R

COUNTY AUDITOR

WOCD COUNTY

ONE COURTHOUSE SO

BOWLING GREEN OH 43402

$419352-6531 \times 2191$

ROSENDALE, RICK

INSPECTOR WEIGHTS \& MEASURES

WOOC COUNTY

316 VINES

BLOMDALE OH 44817

$419352-6531 \times 2191$

\section{YANOCT COUNTY}

GILLISPIE. MIKE

INSPECTOR WEIGHTS \& MEASURES

WYANDOT COUNTY

COURTHOUSE

UPR SANOUSKY. OH 43351

419 294-5212

MBR

NCWM

NCWM

MCCLAIN, JEFF

COUNTY AUDITOR

WYANDOT COUNTY

COURTHOUSE

UPR SANDUSKY。OH 43351

$419294-1531$

CITY: AKRON

ITY SEALANOA

CITY OF CANTON

218 CLEVELAND AVE SW

$216489-3268$

CIRELLID MICHAEL R ${ }^{R}$ MEASURES

INSPECTOR WE

218 CLEVELAND AVE SW

CANTON. OH 44704

FOLTZ, DCUGLAS

INSPECTOR WEICHTS \& MEASURES

CANTON CITY

218 CLEVELAND AVE SW

CANTON: OH 44704

$216489-3268$

CITY: CINCINNATI

LINOSEY, TERRY

INSPECTOR HEIGHTS \& MEASURES

CINC INNATI CITY

3845 WM D DOOL EY BY-PASS

CINC INNAIII

OLEARY JAMES WEIGHTS E MEAS

CITY OF CINCINNATI OHIO

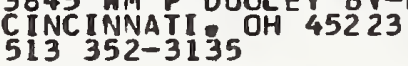

PRAGAR . THOMAS

CHIEF OF WEIGHTS E MEAS

CITY OF CINCINNATI CHIO

3845 WILL IAM P DCOLEY BYPASS

CINCINNATI $\mathrm{OH} 45223$

SCHEER, DI ANE H

WEIGHTS E MEASURES INSPECTOR

CITY OF CINCINNATI CHIO

3845 WM P DOOLEY BY-PASS

$513{ }_{352-3135}{ }^{\text {OH } 45223}$
MBR

NCWM

MBR

MBR

NCWM

MBR

NCWM 
YANKOSKY, JEFFERY

SUPERVISING INSPECTOR W/M

3845 WM P DOOLEY BY-PASS

CINCINNATI OH 45223

$513352-3135$

\section{CITY: CLEVELAND}

GNANN DEBORAH

DIRECTOR

OFFICE OF CONSUMER AFFAIRS

601 LAKESIDE AVE/CITIZENS CTR

CLEVELAND. OH 44114

$216664-3200$

YANCY, JEFFREY

INSPECTOR WEIGHTS \& MEASURES

CLEVELAND CITY

601 LAKESIDE AVE

CLE VELAND OH 44114

CITY: COLUMBUS

CLEM FRED $P$

CONSUMER AF FAIRS INSPECTOR

OFFICE OF WEIGHTS \& MEASURES

50 WEST GAY ST

COLUMBUS $\mathrm{OHH}_{3} 43215$

DIXCN JR, STANLEY

OFFICE CF WEIGHTS AND MEASURES

50 HEST GAY ST

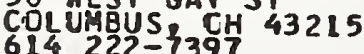

PARKER, CRAIG M

INSPEC TOR WEIGHTS \& MEASURES

50 hEST GAY ST

COL U $222-7397$ SH 43215

ROB INSON CORDELL L

CONSUMER AFFAIRS IN SPECTOR

OFFICE CF HEIGHTS E MEASURES

50 WEST GAY ST

COLUMBUS OH 43215

WALLS, BELINDA

CONSUMER AFFA IRS INSPECTOR

OFFICE OF WEIGHTS E MEASURES

$614222-7397$

\section{CITY: EAST CLEVELAND}

BARNES, HALTER

ASSISTANT SEALER

CITY OF EAST CLEVELAND

EAST CLEVELAND. OH 44112

SCALES JOHN W

BLDG INSPECTOR E COCRDINATOR

14340 EUCLID ITY

216 681-5020

MBR

MBR

NCWM

MBR

NCWM

MBR

MER

\section{CITY: ELYRIA}

PORTER, WILLIAM J

CITY SEALER

CITY OF ELYRIA

ELYRIA. OH 44035

216 322-1829

CITY: EUCLID

VENTURA, SAMUEL

COMHISSIONER HEM

CITY DF EUCLID

585 E 222 ND ST

EUCLID: 289454123

CITY: HAMILTON

LINDSEY TERRY 1

INSPECTOR OF WEIGHTS EMEASURES

CITY OF CINCINNATI

3345 WM P DOOLLEY BY-PASS

C INC INNATI OH 45223

CITY: LORAIN

JACKYM. HALTER F

SEALER WEIGHTS \& MEASURES

LORAIN CITY

508 W 26TH ST

LORAIN. OH 44052

216 245-1691

CITY: NORWOOD

MUMPER • JOHN

SEALER WEIGHTS \& MEASURES

NORHOOD CITY

MONTGOMERY \& ELM STS

$513631-270045212$

CITY: YOUNGSTOWN

CUMMINGS DELORES

ASSISTANY SEALER

CITY OF YOUNGSTOWN

26 S PHELPS ST

$216742-8700$ OH 44503

DULLAN: ANTHONY C

CITY OF YOUNGSTOWN

CITY HALL - 26 S PHELPS ST YOUNGSTOWN: 16 OH 44503

OKL AHOMA

STATE

CARTER, CHARLES D

PROGRAM ADMINISTRATCR WEM AGR ICULTURAL PRODUCTS DIVISICN $2800 \mathrm{~N}$ LINCOLN BLVC

OKLAHOMA CITY ${ }_{405}$ OK 73105

ELL IOTT O RAY

DIRECTOR AGRIC PROCUCTS DIV

OKLA DEPT OF AGRICULTURE

2800 NORTH LINCOLN BLVD

OKLAHCNA CITY OK 73105

$405521-3861 \times 301$ 
EVANS YIRGINIA

ADMINY STRATIVEA ASSI STANT

AGRICUL TURAL PROLUTS OIV

$280 \mathrm{C} N$ LINCOLN BLV

OKLAHOMA CITY, OK 73105

FRALEY KEN

METROLOG IST DIV BU OF STANOARDS

$2800 \mathrm{~N}$ IINCOLN BLVD

OKLAHOMA CITY, OK 73105

$405521-3864 \times 370$

LIPINSK I. THOMAS W

SENIOR MARKETING INSPECTOR

OKL AHOMA DEPT OF AGRICUL TURE

2800 NORTH LINCOLN BLVD

OKLAHOMA CITY 0 OK

SCHCENEKE, ROBERT

SUPERVI SOK REGULATORY SYC

OKLAHOMA DEPT OF AGRICULTURE $280 \mathrm{C} N$ LINCOLN BLVD

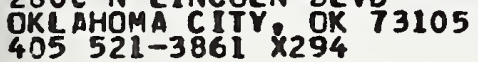

TATE

ARIMA HALTER S

OREGON WEIGHTS \& MEASURES DIV

635 CAPITOL ST NE

SALEM $378-3792$

BAARSTAD, DALE

INSPECTOR WEIGHTS \& MEASURES

ORE GON DEPT OF AGR I CUL TURE

635 CAPITOL ST NE

$503378-3792$

CASNER, DAVID G

OREGON HEIGHTS E MEASURES DIV

635 CAPITOL ST NE

$503378-3792$

CLIFFORD JAMES F

OREGON WEIGHTS \& MEASURES DIV

S35 CAP ITOL ST NE

$503378-3792$

COONEY CLARK

INSPEC YOR WEIGHTS $\&$ MEASURES

OREGON DEPT OF AGR ICULTURE

635 CAPITCL ST NE

$503378-3792$

FOREHAND, RON

INSPECTOR WEIGHTS E MEA SURES

OREGON DEPT OF AGR I CULTURE

635 CAP IIOL ST NE

503 378-3792

FRANOSEN, OEAN

WEIGHTS E MEASURES DIV

OREGON DEPT OF AGR I CULTURE

635 CAP ITOL ST NE

SALEM OR 973
$503378-3792$
MBR

NCWM

MBR

MBR

MBR

PARKS CHR ISTINE A

DEPT OF AGRICULTURE-WTS \& MEAS 635 CAPITAL ST NE

$\begin{aligned} & S A L E M T D R \\ & 503\end{aligned} 78-379210$

ROSS, JAMES E

INSPECTOR

635 CAPITOL ST NE

SALEM. OR 97310

$503378-3792$

SHEFCHECK, GEORGE S

ASSISTANT ADMINISTRATOR

OREGON HEIGHTS \& MEASURES DIV

635 CAPITOL ST NE

SALEM, DR 97310

SIMILA KENDRICK J

ADMINISTRATOR

OREGON HEIGHTS \& MEASURES DIV

635 CAPITOL ST NE

SALEM OR 97310

HYCKOFF, RUSS

INSPECTOR HEIGHTS G MEASURES

OREGON DEPT OF AGR ICUL TURE

635 CAPITOL ST NE

503 378-3792
MBR

NCWM

MBR

NCWM

MBR

NCWH

MBR

NCWM 
LESTER BRENDA J

INSPECTOR

PA BUREAU OF WEIGHTS \& MEASURE $2301 \mathrm{~N}$ CAMERON ST

HARR ISBURG PA 17110

ADAMS, MARGARET S

INSPECTOR OF HEIGHTS E MEASURE

$2301 \mathrm{~N}$ CAMERON ST

HARRISBURG: PA 17110

$717527-4608$

ANGELUCCI, RAYMOND A

PA BUREAU OF HEIGHTS $E$ MEASURE

PA BUREAU OF HEIGHT

HARRISBURG PA 17110

$215586-4958$

8ONNER, ROBERT J

INSPECTOR

PA BUREAU OF WEIGHTS \& MEASURE $2301 N$ CAMERON ST

HARRISBURG PA 17110

$717645-5336$

BRUCKNER, CHARLES M

PASPECTOR OF WEIGHTS \& MEASURE

$2301 N$ CAMERON ST

HAP.P.I SBURG PA 17110

$814763-2823$

CAVANAUGH MICHAEL J

PISTRICT SUP SER WEIGHTS E MEASURE $2301 \mathrm{~N}$ CAMERON ST

HARRISBURG: PA 17110

$215945-9288$

DUDASH JAMES $M$

INSPECTOR

PA BUREAU OF HEIGHTS \& MEASURE

$2301 N$ CAMERON ST

HARRISBURG PA 17110

$717875-3976$

MBR

ELY DEAN F

DIS PRICT SUPERVISOR

332 WASHINGTON AVE

JERSEY SHORE.PA 17740

717 398-2811

FISHER, JOHN E

CHIEF CHEMIST
DEPT OF GENERAL SUCS LAB DIV
2221 FORSTER ST ROOM G-28

HALI FORSTER ST ROOM

$717787-3862$

FOGAL RICKY A

INSPECTOR

PA BUREAU OF WEIGHTS \& MEASURE

$2301 \mathrm{~N}$ CAMERON ST

HARRI SBURG PA 17110

814 942-7383

GUYETTE JR, RCBERT W

PA BUREAU OF WEIGHTS E MEASURE

$2301 N$ CAMERON ST

HARRISBURG PA 17110

$717288-4282$

HOCHREITER, FRANK $P$

IN SPECTCA

PA BUREAU OF WEIGHTS \& MEASURE

$2301 \mathrm{~N}$ CAMERON ST

HARRISBURG: PA 17110

MBR
MAR.TIN. ALLEN H

INSPECTOR

PA BUREAU OF WEIGHTS \& MEASURE $2301 \mathrm{~N}$ CAMERON ST

HARRISBURG PA 17110

$717354-6133$

MAZZIES NICK

INSPECTOR

PA BUREAU OF WEIGHTS E MEASURE

$2301 \mathrm{~N}$ CAMERON ST

HARRISBURG PA 17110

$215252-0262$

MCGOHAN, DONALD I

PA BUREAU OF WEIGHTS \& MEASURE 2301 N CAMERON ST

HARR I SBURG PA 17110

717 787-9089

MENSCH GEORGE

INSPECTOR

PA BUREAU OF WEIGHTS E MEASURE $2301 \mathrm{~N}$ CAMERON ST

HARR I SBURG PA 17110

717 966-1153

MINNITI RICHARD

INSPECTOR

PA BUREAU OF WEIGHTS 6 MEASURE $2301 N$ CAMERON ST

HARRI SQURG PA 17110

$412684-9060$

MONETA. VICTOR M

PNSPECTOR OF WEIGHTS E MEASURE $2301 N$ CAMERON ST

HARR I SBURG. PA 17110

814 965-2097

MORGAN. MARK A

INSPECTOR

PA BUREAU OF HEIGHTS $\&$ MEASURE $2301 \mathrm{~N}$ CAMERON ST

HARRI SBURG PA I7110

$814765-1006$

PAGANO. ANTHONY R

INSPECTOR

PA BUREAU OF WEIGHTS \& MEASURE $2301 \mathrm{~N}$ CAMERON ST

HARR ISBURG PA 17110

$412793-9036$

POTTER JAMES D

PASPECTOR OF WEIGHTS \& MEASURE 2301 N CAMERON ST

HARRISBURG: PA 17110

RAISLEY WAYNE R

INSPEC BUREA OF WEIGHTS E MEASURE 2301 N CAMERON ST

HARRISBURG PA 17110

$412482-4621$

RESICK JR, JOHN J

INSPECTOR OF WEIGHTS \& MEASURE $2301 N$ C.AMERON ST

HAR.RISBUPG PA 17110

$412568-3770$ 
RIGHT STANLEY SUPATSOR

PA BUREAU OF WEIGHTS \& MEASURE HARRI ISBURG PA 17110

$814398-4033$

ROCF, RCN

ASSISTANT OIRECTOR

PA BUREAU OF HEIGHTS \& MEASURE $2301 N$ CAMERON ST

HARR ISBURG PA 17110

$717787-9089$

SEROVICH, THOMAS J

INSPECTCR TH HEIGHTS \& MEASURE

PAON CAMERON ST

HARRISBURG. PA 17110

$717339-4401$

THOMAS, FRED A

OIRFCTUR OF WEIGHTS \& MEASURE

2301 N CAMERON ST

HARR ISBURG PA 17110

$717787-6772$

WEOGE CHARLES A

IN SPECTOR

PA BUREAU OF WEIGHTS \& MEASURE

2301 N CAMERON ST

HARRISBURG PA 17110

$4125,38-5618$

WINKLER: JOSEPH V

INSPECTOR OF HEIGHTS \& MEASURE

PAO N CAMERON ST

HARRISBURG PA 17110

215 533-6984

\section{IDAMS COUNTY}

BENNER ${ }^{0}$ EDWIN

ADAMS COUNFY H E $M$

230 BENNER RD

GETTYSBURG PA 17325

$717334-1769$

\section{ALLEGHENY COUNTY}

BELL, JACK

CHIEF INSPECTOR

PENN LIBERTY PLZ 1520 PENN AVE 412 P92-8576

\section{ARMSTRONG COUNTY}

BALSONE JOSEPH D ${ }^{D}$ AR $\&$ M

2233 MANOR DR

FORD CITY. PA 16226

$412763-7433$

\section{BEAVER COUNTY}

PRESUTT I RUDY

BEAVER COUNTY H E M

1004 DELOUSKE AYE

MI DLAND. PA 15059

$412728-5700 \times 421$

\section{BEDFORD COUNTY}

UHL AND, JOHN

BEDFORÓ COUNTY $W \varepsilon M$

$R D 1$

HOPEWELL, PA 16650

$814623-1173$

\section{BLAIR COUNTY}

SHAWLEY, JOHN D

BLAIR COUNTY H E M

1401 HALNUT ST

HOLLIDAYSBURG, PA 16648

B14 $695-5541$

MBR

NCHM

BRADFORD COUNTY

COOK NORMAN

BRADFORO COUNTY W G

COURTHOUSE

TOWANDA. PA 18848

717 265-5700

BUCKS COUNTY

MBR

NCWM

ADAMS PEGGY H

CHIEF SEALER

BUCKS CTY CONSUMER PROTECTION

BROAD AND UNION ST

DOYLESTOHN. PA $189 \mathrm{CI}$

$215348-7442$

BO220. CHARLES

DEPUTY SEALER

BROAD E UNION STREETS

DOYLESTOWN. PA I8961

$215348-7442$

CONKLIN: RUSSELL

DEPUTY SEALER

DEPT OF CONSUMER PRCTECTION

BROAO \& UNION STREEIS

DOYLESTOWN. PA 18901

$215348-7442$

DI GUGLIELMD. NICHCLAS

DEPUTY SEALEF CONSUMER PRCTECTION BROAD E UNION STS

DOYLESTOWN PA 18901

$215-348-7442$

MBR

MIRACHI E EWARD

DEPUTY SEALER

DEPT OF CONSUMER PRCTECTION

NCHM

BROAO UNION STREETS

DOYLESTOWN. PA 18901

$215348-7442$

RUGGHIA ANTHONY

DEPUTY SEALER

MBR

BROAD E UNION STREETS

DOYLESTOWN PA 18901

215 348-7442

\section{BUTLER COUNTY}

HOQVER MARTIN J

71 HORTON AVE

BUTTLR PA 16001

$412285-4731 \times 259$

CAMBRIA COUNTY

SHARBAUGH。 ALBERT

CAMBR IA COUNTY HEM

I34 ST MARY ST

CARROLLTOWN. PA 15722

$814344-6565$
MBR 
NARBY CAMERON MAXWELL \& M

301 S MAPLE ST

EMPORIUM. PA 15834

814 483-1014

CARBGN COUNTY

HUNSICKER O WAYNE E H

COURTHOUSE

JIMT THORPE PA 18229
717 325-3611

CENTRE COUNTY

MBR

CRUST. RONALO R.

CENTRE COUNTY W $E M$

$B O X 574$

MILESRURG PA 16853

CHESTER CCUNTY

TAYLOR ROBERT N

CHESTER COUNTY W E M

WEST CHESTER, PA 19382

NCWM

\section{CLARICN COUNTY}

MORTIMER GREGORY $K$

CLARION COUNTY H $6 M$

BOX 387

RIMERSBURG, PA 16248

614 473-6267

\section{CLEARFIEL O COUNTY}

BEKAYSA FRANK

CLEARFIELD COUNTY $W \& M$

COALPORT PA 16627

$814765-6546$

\section{CLINTCN COUNTY}

GREENAWAY CARLTON J

EASTERN CLINTON COUNTY W $\& M$

RFD 1 BOX 397

JERSEY SHORE, PA 17740

$717753-3849$

HANDBIC PHYLLIS

CLINTON COLNTY H

RENCVA, PA 17764

G $M$

KLEWANS SYLYAN

325 N WATER ST W E.

717 MILL-4927 17751

\section{COLUARIA COUNTY}

KOVACH DAVID M

COLUMBIA COUNTY W E M

817 MUL BERRY ST

BERWICK PA 18603

$717752-7166$
LEBLANC RIC

CUMBERLANO COUNTY $\& M$

CUMBERLAND CO CRTHSE/COMM OFF

CARLISLE PA 17013

$717697-0371 \times 239$

DAUPHIN COUNTY

COOPER WILLIAM S

DAUPHIN COUNTY $\& M$

FRONT \& MARKET ST

HARRISBURG PA 17101

$717255-2944$

DELAHARE COUNTY

BALLE JAMES

COUNTY OF DELAWARE

CONSUMER AFFRS/TOAL BLDG MEDIA. PA 19063

$215891-4865$

CLQUO. RICHARD

ASST TO CHIEF SEALER

BUREAU OF WHTS E MEAS

GOVERNMENT CENTER BUILDING MEDIA. PA 19063

$215891-4865$

DONAHUE MICHAEL

DEPUTY SEALER 2 WGTS $\&$ MSRS GOVERNMENT CENTER BUILDING MEDIA. PA 19063

$215891-4865$

STUBBS. JOHN

DEPUTY SEALER

CONSUMER AFFRS \& WGTS \& MSRS GOVERNMENT CENTER BUILDING MEDIA. PA 19063

$215891-4865$

YANCOSKIE, EVELYN $M$

DIRECTOR \& CHIEF SEALER CONSUMER AFFRS \& WGTS \& MSRS GOVERNMENT CENTER BUILDING MEDIA. PA 19063

$215891-4865$

ELK CDUNTY

GEORGEVICH GEORGE

ELK COUNTY W E 226 RUSS LANE ST MARYS PA 15857

$814766-1161$

ERIE COUNTY

BLASCD JOSEPH C

ERIE COUNTY W E M 140 W $6 T H$ ERIE PA 16501

$814452-3333 \times 359$

\section{FAYETTE COUNTY}

BEZILLA. BERNARD

FAYETTE COOUNTY W $\& M$

COURTHOUSE

UNIONTOWN. PA 15401

$412437-1315$ 


\section{ANKLIN COUNTY}

THCMPSON JOHN J

FRANKLIN COUNTY W $W M$

COURTHOUSE

CHAMBER SBURG.PA 17201

717 264-4125

ILTCN COUNTY

BARNHART BERNARD $P$

FULTON COUNTY $W$ G $M$

NEEDMORE PA 17238

$717573-2389$

REENE COUNTY

CVETAN MICHAEL $P$ 7 RIVER VIEW AVE BOX 13 $412592-5120$

MB R

NOIANA COUNTY

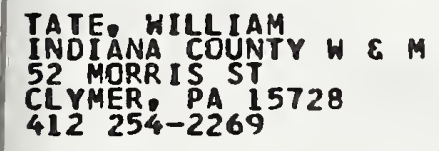

EFFERSON COUNTY

\section{BISH CHARLES E \\ JEFFERSON COUNTY W $\& M$ \\ RD 1 \\ SUMMERVILLE
814 PA $1586-2135$}

\section{UNIATA COUNTY}

BDOK ROY D

JUNIATA COUNTY H E M

BOX 372 RD 1

MIFFLINTCWN:

PA 17059

-ACKAWANNA COUNTY

VESNESKY。 LEONARD

LACKAWANNA COUNTY W $E M$

1513 CHURCH AVE

STRANTON PA 18508

\section{LANCASTER COUNTY}

CLIFF. S KENNETH BUREAU CF WEIGHTS S MEASURES 50 N DUKE ST PO BOX 3480

LANCASTER, ${ }^{\circ A} 17603$

\section{LAWRENCE COUNTY}

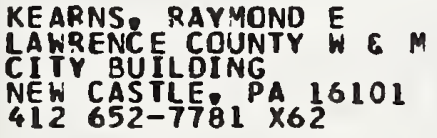

\section{LEBANON COUNTY}

DISSINGER, MARVIN $R$ LEBANDN COUNTY W E M RM 207 MUNICIPAL BLCG LEBANON,PA 17042

$717274-2801$

\section{LEHIGH CCUNTY}

MAHONEY \& DONALD \& 260 S CEDARBROOKK RD ALLENTOHN. PA 18103 ALLENTOHNB 215 P

\section{LUZERNE COUNTY}

\section{MCNULTY. RAYMOND}

LUZERNE COUNTY WE $M$ WILKES-BARRE. PA 18702 WILKES-BARRE

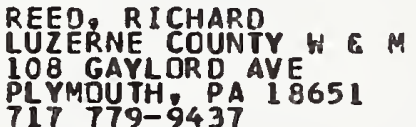

L YCOMING COUNTY

GERADI JOHN A

LYCOMING COUNTY 144 HUFFMÁN AVE

HILLIAMSPORT. PA 17701

$717323-7312$

GREEN. GUY

LYCOMING COUNTY H \& R D $A 1$ BOX 236 HUGHESVILLE: PA 17737 $717584-2269$

JENKINSE CARL E H BOX 253 COCAN STATION. PA 17728 $717998-2995$

MCKEAN COUNTY

MCKECHNEY, JAMES M MCKEAN COUNTY H \& 117 S FRALEY ST

KANE PA 16735

$814837-8088$

MERCER CDUNTY

HOFFMAN, HAROLD F

MERCER COUNTY W \& $M$ 58 MORTON ST $4{ }_{41}$ AT $342-9757$ PA 16161

MIFFL IN COUNTY

FIKE, EDWARD D

MIFFLIN COUNTY W $E M$ RD 4 BOX 302

LEWISTOWN PA 17044

717 248-0254 
BLUM RCBERT T

MONRCE CCUNTY H E $M$

RO \& BOX 8097

STRUUDSEURG: PA 18360

FLICK, ROBERT

MONROE COUNTY WE $M$

MBR

EAST STROUDSBURG.PA 18301

$717421-5745$

MONTGCMERY COUNTY

EWING JRE SAMUEL J

CDUNTY SEALER

COURTHOUSE

NORRISTOWN 215 PA 19404

HEFFENTRAGER, EARL S CHIEF SEALER EATS
BUREAU CF HEIGHTS $E$ MEASURES
COURT HOUSE

NORRISTOUN $215278-356919404$

MB R

NCWM

PETERSON JOHN J

WEIGHTS AND MEASURES

COURTHOUSE

NORRISTOHN 215 PA 19404

MONTOUR CCUNTY

BUCKLEY. GERALD R

MONTOUR COUNTY $W$ \& $M$

RD 3 BOX 120

OANVILLE PA 17821

MORTHAMPTON COUNTY

DAVIS, JAMES $R$

NORTHAMPTON COUNTY W E $M$

EASTON PA 18042

$215253-4111 \times 306$

DAVIS JAMES R

NOR THAMPTON COUNTY W E M

TTH \& WASHINGTON STS

EASTON: PA 18042

$215253-4111 \times 306$

NORTHUMBERLAND CDUNTY

VEECH JR GUS

NORTHUMBERLAND COUNTY W E M 530 SPRUCE ST

T17 P73-1 PA 17834

PERRY CDUNTY

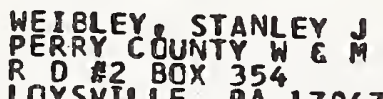

LOYSVILLE $P$ S

$717582-2636$

MBR

NCWM
QUINN. MERRITT B

PIKE COUNTY H E

$306 \mathrm{~W}$ ANN ST

MILFORO PA 18337

\section{POTTER COUNTY}

TAVENIER , FRED

POTTER COUNTY W E

BOX 192

ROULETTE, PA 16746

$814544-7528$

SCHUYLKILL COUNTY

KITSOCK, METRO K

SCHUYKILL COUNTY W E H

147 DGDEN ST

GIRARDVILLE: PA $17 \$ 35$

$717276-6689$

\section{SNYDER COUNTY}

WENTZEL II JAMES $B$

WEIGHTS \& MEASURES

DEPT OF AGRICULTURE

RD 3 BOX 104

SELINSGROVE: PA 17870

SOMERSET COUNTY

IRHIN, WILLIAM

SOMERSET COUNTY H E $M$

42 SAL I SBURY ST

MEYERSDALE PA 15552

814 445-7991

\section{SUSOUEHANNA COUNTY}

TREYAN: JOHN

SUSQUEEHANNA COUNTY \& $\varepsilon M$ 822 SUSQUEHANNA ST

FOREST CITY. PA 18421

TIOGA COUNTY

WIL SON. GEORGE

IIOGA COUNTY W E M

BLOSSBURG, PA 16912

717 724-1906

UNION COUNTY

REEOY CHARLES E

UNION COUNTY W E M

RO 3

LEWISBURG, PA 17837

VENANGO COUNTY

RHODES, RICHARD E

VENANGÓ COUNTY WEM

119 E 5 TH ST

814 CITY P PA 16301

WARREN COUNTY

SANDEN - HERBERT W

HARREN COUNTY W $G$

29 W 320 AVE

WARREN: PA 16365

$814723-7550^{\circ}$ 
ME S SEROTES GERGE

WASHING TON COUNTYW E M 404 CASHING TON. PA 15301

$412228-6866$

\section{PETRICCA, ED}

JEIGHTS MEASURES

DEPT OF AGRICULTURE

COUNTY OFF BLD ROOM 404

WASHING TON PA 15301

$412228-6866$

AYNE COUNTY

\section{MANG, HAROLD \\ HAYNE CCUNTY WE \\ RAD \\ HONEŚDALE, PA 18431 \\ $717253-5970$}

\section{ESTMOREL AND COUNTY}

CAPCZZI \& HELEN E

BUREAU OF WEIGHTS AND MEASURES

RD 12 BCX 203

412 836-54LO

CATCNE EMILL

BUREAU OF WEIGHTS AND MEASURES

RD $12 \mathrm{BOX} 203$

GREENSBURG PA 15601

$412836-5410^{\circ}$

LEONE: THOMAS A

DUREAU OF WEIGHTS AND MEASURES

RD 12 BOX 203

GREENSBURG PA 15601

$412836-5410$

WYOAING COUNTY

CRUYER, EYELYN R

RYO ING COUNTY $H E$

LACEYVILLEE PA 18623

$717869-1370$

YORK COUNTY

BROWNE CLENN

YORK COUNTY W $E M$

YWEST MARKET WAY

YORK $P A$ A 17401

717 B $48-3301 \times 265$

CITY: ARNDLD

HOPKINS • ROBERT J

CITY OF ARNOLD W $E$

1600 KIMBALL AVE

ARNCLD, PA 15068

$412337-4402$

CITY: BETHLEHEM

MEYERS, DAYIO

CITY OF BETHLEHEM $W$ E $M$

IO E CHURCH ST CITY CENTER

215 B65-70์93

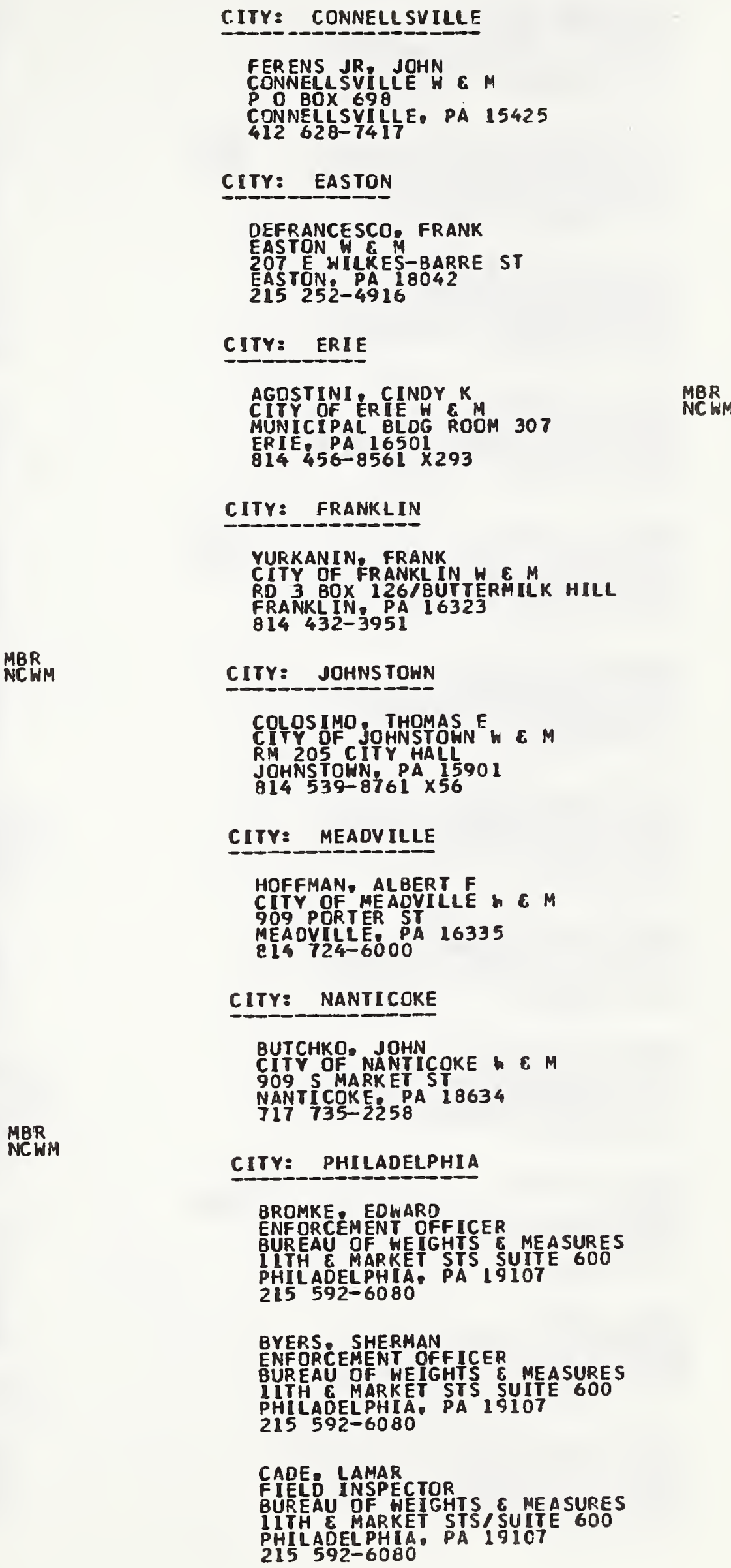

MBR
NCWM

CITY: JOHNSTOWN

COLOSIMO THOMAS E

RM 205 CITY HALL

JOHNSTOWN PA 15901

$814539-8761^{\circ} \times 56$

CITY: MEADVILLE

HOFFMAN. ALBERT F 909 PORTER ST

MEADVILLE: PA 16335

CIS 724-6000

CITY: NANTICOKE

BUTCHKO, JOHN

CITY OF NANTICOKE $\& M$ 909 S MARKET ST NANTICOKE 735 PA 18634

MBR

\section{CITY: PHILADELPHIA}

BROMKE E EWARD

ENFORCEMENT OFFICER MEASURES IITH \& MARKET STS SUITE 600 PHILADELPHIA. PA 19107 215 592-6080

BYERS. SHERMAN

ENFORCEMENT OFFICER

BUREAU OF WEIGHTS \& MEASURES

IITH $M A R K E T$ STS SUITE 600

PHILADEELPHIA. PA 19107

$215592-6080$

CADE

FIELD INSPECTOR

BUREAU OF WEIGHTS $E$ MEA SURES 11THE MARKET STS/SUITE 600 PHILADEL PHIA, PA 19107

$215592-6080$ 
CURRENCE CARL

BUREAU OF WEIGHIS \& MEASURES IITH \& MARKET STS SUITE 600 PHILADELPHIA. PA 19107

$215592=6080$

DAVIS. PAUL

FI ELD I NSPECTION SUPERVISOR BUREAU OF WEIGHTS \& MEASURES ONE READING CENTER

PHILADELPHIA. PA 19107

215 592-6079

GOCDJIONES, RONALD

ENFORCEMENT OFF ICER

BUREAU OF HEIGHTS \& MEASURES

IITH \& MARKET STS SUITE 600

PHILAOELPHIA. PA 19107

215 592-6080

SONES CARGLYN

BUELD INSPECTOR IITH \& MARKET STS/SUITE 600 PHILADELPHIA. PA 19107

$215592-6080$

KILLEN, CAROLINE

SUPERVISOR

BUREAU OF WEIGHTS \& MEASURES

IITH \& MARKET STS SUITE 600

PHILADELPHIA. PA 19107

215 592-6079

KING GEORGE

ENFCRCE MENT OFFICER

BUREAU OF WEIGHTS \& MEASURES

IITH E MARKET STS SUITE 600

PHILADELPHIA, PA 19107

$215592-6080$

MCLAUGHLIN: HALTER

FIELD INSPECTION SUPER VISOR

ONE READING CENTER

PHILADELPHIA.PA 19107

215 592-6079

MICKEY, JOSEPH

ENFCRCEMENT OFFICER

BUREAU OF HEIGHTS \& MEASURES

IITH \& MARKET STS SUITE 600

PHILADELPHIA. PA 19107

$215592-6080$

MOORE, THOMACINA

ENFORCEMENT OFFICER

BUREAU OF WE IGHTS \& MEASURES

1 ITHE MARKET STS SUITE 600

PHILADELPHIA. PA 19107

MURPHY, EMRETT

CHIEF

PHILADELPHIA WEIGHTS $E$ MEASURE

MBR

11 TH \& MARKET STS SUITE 600

PHILADELPHIA, PA 19107

215 592-6000

NOONE, JAMES

ENFCRCEMENT OFFICER

BUREAU OF MEIGHTS MEASURES

1ITH E MARKET STS SUITE 600

PHILADELPHIA.
215 592-6080

OWENS STEVEN

ENFCRCEMENT OFFICER

BUREAU OF WEIGHTS \& MEASURES

1ITH MAP.KET STS SUITE 600

PHILADELPHIA. PA 19107

$215592-6080$

MBR
MER

NCWM

NCHM

ENF JR A ALBER

ENFORCEMENT OFFICER

BUREAU OF HEIGHTS \& MEASURES

PHILADELPHIA, PA 19107

215 592-6080

TAREILA: MICHAEL

BIELEDINSPECTOR BUREAU OF HEIGHTS SUTEASURE PHILADELPHIA. PA 19107 $215592-6080$

TULLY JR EDWARD

ENFORCEMENT OFFICER

BUREAU OF WEIGHTS \& MEASURES

IITH E MARKET STS SUITE 600

PHILADELPHIA. PA 19107

215 592-6080

HALKER PERCY

FIELD INSPECTOR

BUREAU OF WE IGHTS $E$ MEA SURES

IITH \& MARKET STS/SUITE 600 PHILADELPHIA. PA 19107

215 5.92-6080

WOLSTENHOL ME, WILL IAM

ENFORCEMENT OFF ICER

BUREAU OF WEIGHYS \& MEASURES

IITH E MARKET STS SUITE 600

PHILADELPHIA. PA 19107

215 592-6080

CITY: PITTSTON

GIAMBER, SAMUEL

PITTSTON H $\&$ M

100 JOHNON ST

PITTSTDN PA 18640

$717654-0513$

CITY: SCRANTON

HAZZOUR I JOHN J

SCRANTON H \& M

340 N WASHINGTON AVE

SCRANTON PA 18503

$717348-4194$

CITY: SHARON

KRESS, HENRY

CITY OF SHARON W 6

155 CONAELLY BLVD

SHARON PA 16146

$412981-5550$ 


\section{:IY: UNICNTOWN}

ING SR . HARVEY C

INICNTOUN $H$ \& $M$

iNICNTOWN, PA 15401

$12437-3211$

CITY: WILKES-BARRE

ALLABAUGH, GARY

ITYY HALL

BUUR OF ENGI NEER ING

WILKES-BARRE.PA 18702

$717826-8247$

\section{QR.ICO}

\section{SATE}

COLON EOUARDO A ME SURES DIV DEPARTMENT OF CONSUMER AFFAIR

SANTURCE PR 00940

$809726-7555 \times 3668$

COLCN RENE A

ADVISER COMMITTEE/AGRICULTURE

SENATE OF PUERTO RICO

CALLE BARBE 504

SANTURCE PR OO912

$809725=9196$

KWILAN IBONE

OEPARTMENT OF CONSUMER AFFAIRS PO BOX 41059 MINILLAS STATION SANTURCE, PR 00940 $809726-7585$

\section{ORTIZZ ANA V ASST SECY OFUR CONSUMER AFFAIRS PO BOX 41059 MANILLAS STA SANTURCE PR 00940 $809721-1910$}

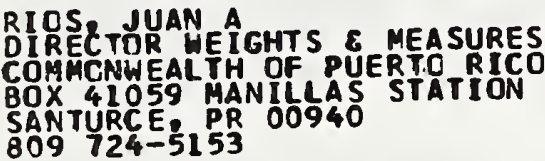

RIOS, JUAN A DIRECTOR HEIGHTS $\&$ MEASURES COAMCNWEALTH OF PUERTO RICO BOX 41059 MANILLAS STATION SANTURCE 8 P PR

ROSARID-FRED, EOMONDO METROLOGIST DEPT CONSUMER AFFAIRS PR $P O B O X$ \& 1059 MANILLAS STATION SANTURCE PR 00940 $726-7220$

\section{ISLAND}

\section{STATE}

MAURER LYNDA

SUPERVI SING METROLOGIST

220 ELMWOOD ROAD PROVIDENCE RI 02907

$401457-1863$

\section{CITY: BARRINGTON}

SPEAKER • ROBERT B

WE IGHTS \& MEASURES

RI DEPT CF LABOR

BARRINGTCN. RI 02806

\section{CITY: BRISTOL}

PROTO, EDUARD

WEIGHTS E MEASURES

RI DEPT OF LABOR

II GOULANT AVE

BRISTOL. RI 02809

CITY: CENTRAL FALLS

GOES JR, MANUEL

HEIGHTS 5 MEASURES

RI DEPT OF LABDR

22 SAMOSET AVE

CENTRAL FALLS. RI 02863

CITY: CRANSTON

RONNCCI. CAROL

WEIGHTS $G$ MEASURES

RI DEPT OF LABOR

4 CAPORAL ST

CRANSTON. RI 02910

CITY: CUMBERLANO

HALAJKO. PETER

WEIGHTS \& MEASURES

RI DEPT OF LABOR

181 ANN ST

CUMBERLAND. RI 02864

CITY: EAST GREENWICH

FIT ZPATRICK. ROBER

HEIGHTS \& MEASURES

RI DEPT OF LABOR

PO KINGSTOWN. RI 02852

401 295-7754

NBR

CITY: EAST PROVIDENCE

SMITH JACK

WEIGHTS 6 MEASURES

RI DEPT OF LABOR

28 COVE ST

CITY: FOSTER

MBR

MERH

MCKENNA- HILLIAM

WEIGHTS \& MEÁSURES

RI DEPT OF LABOR

FOSTER.RE 02825

CITY: HOPKINTON

AHERN. MAJORIE

WEIGHTS \& MEASURES

RI DEPT OF LABOR

KUEHN RD

HOPKINTON, RI 02833

\section{CITY: JAMESTOWN}

MOR INKO • EDWARD

HEI GHTS \& MEASURES

RI DEPT OF LABOR

31 HOWL AND AVE

JAMESTOWN. KI 02835 


\section{CITY: JOHNSTON}

MCCARRON. JAMES

WE I GHTS E MEASURES

RI DEPT OF LABOR

29 EROWN AVE

JOHNSTDN.RI 02919

\section{CITY: LINCOLN}

RICAS RD, MICHAEL

I LAPORTE AVE

MANVILLE。 RI 02838

CITY: LITTLE COMPTON

COLE JAMES $F$

WEIGHTS 6 MEASURES

RI DEPT OF LABOR

OLDMAIN ROAD

CITY: MIDLLETOWN

\section{KEMBLE HALTER}

WEIGHTS \& MEASURES

RI DEPT CF LABOR

4 KING RD

MIDDLETOWN. RI 02840

\section{CITY: NARRAGANSETT}

MURPHY, NEIL

WE IGHTS \& MEASURES

RI DEPT OF LABOR
32 NORTH HILL VIEW OR
NARRAGANSETT RI 2882

CITY: NORTH KINGSTOWN

FI TZPATRICK, ROBERT

SEALER OF WEIGHTS \& MEASURES NORTH KINGSTOWN

P O BOX 24

NORTH KINGSTOWN, RI 02852

401 295-7754

CITY: NORTH PROVIOENCE

TESTA: JCHN

HE IGHTS \& MEASURES

RI DEPT OF LABOR

73 CHARLCTTE ST

NORTH PRCVIDENCE, RI 02904

CITY: NORTH SCITUATE

TUCKER, RAYMOND

WEICHTS \& MEASURES

CENTRAL AVE/RFD BOX 140

NORTH SCITUATE. RI 02857

CITY: NORTH SMITHFIELD

FREITAS - JOSEPH

WEIGHTS \& MEASURES

RI DEPT OF LABOR

FREITAS LANE

NORTH SMITHFIELD。RI 02895

\section{CITY: PAWTUCKET}

KENNEDY. LEO

WEIGHTS E MEASURES

RI DEPT OF LABOR

I6 AMTUY ST. RI 02861

CITY: PORTSMOUTH

WILKEY, DOUGLAS

WEIGHTS $\&$ MEASURES

RI DEPT CF LABOR

174 MIDDLE RD

PORTSMOUTH。RI 0287I

CITY: PROVIDENCE

ALMAGNO PHILIP A

CHIEF UTILITIES E STANDARDS

INSPECTION E STANDARDS DEPT

112 UNION ST

PROVIDENCE:RI 02903

CITY: SMITHFIELD

KEMP. STEVEN

WEIGHTS \& MEASURES

RI DEPT OFF LABOR

2 DOUGLAS CIRCLE

CITY: SOUTH KINGSTOWN

LAVALLEE NORAAN

WEIGHTS \& MEASURES

RI DEPT OF LABOR

1 STEOMAN RD

SOUTH KINGSTOWN, RI 02852

MBR

NCWM

CITY: TIVERTON

WHITE DEBRA

WEIGHTS \& MEASURES

RI DEPT OF LABOR

23 MULBERRY LANE

CITY: WARREN

SYLVIA, MANUEL

HEIGHTS $\&$ MEASURES

RI DEPT OF LABOR

872 MAIN ST

WARREN. RI 02885

CITY: WEST GREENHICH

LAMERE. GEORGE

WEIGHTS \& MEASURES

RI DEPT DF LABOR

WICTORY HWY

CITY: WEST WARWICK

TESSI TOR.E. JOHN

WFIGHTS \& MEASURES

RI DEPI OF LABDR

13 PENTO ST

HEST WARHICK, RI 02893 
PEPIN MAURICE D CITY SEALER OF HEIGHTS MEAS 169 MAIN ST WOONSOCKEY ${ }^{R I} 02895$

ICAROLINA

TATE

ALEXANDER \& $B$

WEIGHTS \& MEASURES OF AGRIC PO BOX 11280

COLUMBI A SC 29211

ALEXANDER , MARYIN

WEIGHTS E MEASURES $P$ BOX 11280

COLUMBIAA SC 29211

$803737-2.080$

BAIRD, IVEY

HE I GHTS E MEASURES

SOUTH CAROLINA DEPT OF AGRIC P O BOX 11280

COLUMBIA SC 29211

BROWN NEAL

WEIGHTS $\&$ MEASURES P O BOX 11280

COLUMBIA 803 T37-2080 29211

CAUSEY, ALAN

WEIGHTS \& MEASURES $P$ BOX 11280

COLUMBIA. SC 29211

$803737-2080$

DAYIS. EILL

WEIGHTS E MEASURES PO O BOX 11280

COLUMBI A SC 29211

FRAZIER - JAMES $R$

WEIGHTS $E$ MEÄSURES

SUUTH CAROLINA DEPT OF AGRIC $P$ C BOX 11280

COLUMBIA $A_{8} S C 29211$
$903737-2080$

FRAZIER THOMAS E

WEIGHTS E MEASURES P 0 BOX 11280

COLUMBIA SC 29211

$803737-2080$

FULAER, CAROL $P$

ASSISTANT DIRECTOR

SC DEPT OF AGR ICULTURE

P O BCX 11280

COL UMBI A. SC 29211

$803737-2080$
GEDOIS: TONNY

WEIGHTS $\&$ MEASURES

SCUTH CAROLINA DEPT OF AGRIC P 0 BOX 11280

COLUMBIA SC 29211

GILLIAM, CHARLES A

FIELD SPECIALIST SUPERVISOR SC DEPT OF AGR I CUL TURE

PO BOX 11280

COLUMBIA SC 29211

GRAYSON- HARYEY

WEIGHTS $\varepsilon$ MEASURES

SOUTH CAROLINA DEPT OF AGRIC P 0 BOX 11280

$803737-208029211$

GREGORY JACK

HEIGHTS $\&$ HEASURES

SOUTH CAROLINA DEPI CF AGRIC P 0 BOX 11280

COLUMBIA: SC 29211

KENNINGTON BILLY

SEIGHTS \& MEASURES T OF AGRIC SOUTH COX II 280

POLBR I1 80
COLUMBIA 29211
$803737-2084$

KOON. MARY FRANCIS

3000 LOUISA ST

COLUMBIS SC 29204

LEE JR: A $Y$

WEIGHTS E MEASURES

SOUTH CAROLINA DEPT OF AGRIC P 0 BOX 11280

COLUMBIA 303 SO 29211

LUNOY LLOYD

WEIGHTS E MEASURES P 0 80X 11280

COLUMBIA SO 29211

NANCE, FRED $M$

WEIGHTS E MEASURES $P Q B O X 11280$

COLUMBIA SC 29211

PUGH, JOHN Y $S$ C DEPT DF AGRICULTURE

$P O B O X 11280$

COLUMBIA SC 29211

REDD JOHN C

WEIGHTS \& MEASURES

SOUTH CAROLINA DEPT OF AGRIC $P 0$ BDX 11280

MBR COLUMBIA SC 29211

RITTER\& L R

WEIGHTS E MEASURES CAROLINA DEPT OF AGRIC P 0 BOX 11280 COLUMBIA
MBR

NCWM 
SAL VO TED

WEIGHTS E MEASURES CARDLINA DEPT OF AGRIC P C BCX 11280

COLUMBIA: SC 29211
$803737-2080$

SHORT, JAMES M

WDUGHTS E MEASURES PO BOX 11280

COLUMBIAAS SC 2921

\section{SHORTER, RAYMOND}

SOUTH CAROLINA DEPT OF AGRIC $P$ BOX 11280

COLUMBIA SC 29211

SLIGH JAMES D

WE IGHTS $E$ MEA SURES

SOUTH CAROLINA DEPT OF AGRIC P 0 BOX 11280

COLUMB IA: SC 29211
$803737-2080$

TERRY, THOMAS C

WWEUTH CAROMEASURES $P 0$ BOX 11280

COLUMBIA SC 29211

WARREN, TOMMY

WEIGHTS $\&$ MEASURES

SOUTH CAROLINA DEPT OF AGRIC (1)

COLUMBIA SC 29211

WILBURN, JACK T

WEIGHTS \& MEASURES

SOUTH CAROL INA DEPT OF AGRIC 80 90X 11280

CDLUMEIA SC 29211

WILLIAHS, ERIC

SEIGHTS E MEEASURES OF AGRIC $P 0$ BOX 11280

CULUMAIA SC 29211

WILLIAMS. RICHARD A

WEIGHTS \& MEASURES

SOUTH CAROL INA DEPT OF AGRIC P BOX 11280

COLUMBIA SC 29211

WILSON GEORGE A

WE IGHTS \& MEASURES

SOUTH CAROLINA DEPT OF AGRIC P O BOX 11280

$803737-208029211$

WOL.FE, ROBERT

WE GHJS 6 MEA SURES

SOUTH CAROLINA DEPT OF AGRIC 0 BDX 11280

COLUMB IA SC 29211

WR IGHT: SAM C

WEIGHTS \& MEÁSURES

SOUTH CAROLINA DEPT OF AGRIC

$P 0$ BOX 11280

COLUMBIA SC 29211
STATE

BIES, LEONARD G

WEIGHTS MEASURES INSPECTOR III

SD DIVICOMMERC IAL INSP \& REG

4109 S FAIRHALL

SIDUX FALLS: SD 57106

BUSH. RALPH

HEAVY SCALE INSPECTCRS

COMMERCIAL INSPECTION \& REG

3309 WISCONSIN AVE

RAPIO CITY SO 57701

CREAGER , MARK G

RETAIL INSPECTOR

COMMERCIAL INSPECTION \& REG

RR 11 BOX 130

${ }_{605}$ RAP CITY SD 57702

FOSTER B BOB

REXAIL INSPECTORS

COLMAN, SO 57017

LINGOR. PAUL

RETAIL INSPECTOR

COMMERCIAL INSPECTION \& REG $111112 \mathrm{TH}$ AVE NE $605225-140757401$

MELGAARD. JAMES

DIRECTOR

OIV COMM INSP \& REG

118 HEST CAPITOL PIERRE: SD 57501

OSTERKAMP, RENEE

EXECUTIVE ADMINISTRATIVE SEC COMMERCIAL INSPECTION E REG PIERRE $773-3697$

PESKY. JIM

SENIOR RETAIL INSPECTOR

COMMERCIAL INSPECTICN E REG

BOX 323

HARRISBURG SD 57032

SCHMIDT, ROGER

HEAVY SCALE INSPECTCR

OIV OF COMH INSP E REGULATION

RR $1 \mathrm{BOX} 72$ WILLOW LAKËE SO 57278

WALZ. DON

RETAIL INSPECTOQ

DIV OFF COMM INSP E REGULATION

PARKSTON

SD 57366

Ni

No

NE

ME

TENNESSEE

STATE

WELL i ${ }^{B}$ EE Pr OF AGRIC

DIV OF MARKETING

PO BOX 40627 MELROSE STA

NASHVILLLE TN 37204 
BI CKEL 3 CHARLES

W.

PO BOX 40627 JMELROSE STA NASHVILLE TN 37204 $615728=7406$

CARRDLL JACKSON F

W.

DIV OF MARKETING

PO BOX 40627 JMELROSE STA

NA SHVILLE TN 37204

$615282-4546$

CLOYD. GARY YN AGRIC

DIV OF MARKETING

PO BOX 40627/MELROSE STA

NASHVILLE. TN 37204

DRINNON: KENNETH D

AREA SUPER VISOR

WO EM DEPT OF AGRIC

NASHVILLE. TN 37204

$615475-2564$

EDDE CHARLES

METROLOGIST

DEPT OF AGRICULTURE IDIV MKTG P O BOX 40627 MELROSE STATION NASHVILLE. TN 37204

$615360-0158 \times 39$

EDMUNDSCN DONALD M

W E M DEPT DF AGR

PO BOX 90627 JMELROSE STA

NASHVILLE: IN 37204

$901885-2610$

FREEMAN RICKEY J

AREA SUPERYISOR

DIVISION OF MARKETING

P O BOX $40627 / M E L R O S E$ STATION NASHVILLE, TN 37204

$901692-2059$

GAINES, JOE $R$

DIRECTOR

WE IGHTS AND MEASURES

BOX 40627 MELROSE STATION

NASHVILLE TN 37204

HARRI SON: ERNEST L

W $E$ M DEPT DF AGRIC

PÓ BOX $40627 / M E L R O S E$ STA

NASHUILLE: IN 37204

$615937-8719$

HODGE, HAL C

WE MDEPT CF AGRIC

DIV OF MARKETINC

PO BOX 40627 JMELROSE STA

NA SHVIL LE. TN 37204

$615397-2240$

IVENS PEID

W. M OEPT OF AGRIC

DIY OF MARKETING

PO $80 \times 40627$ / MELRCSE STA

NASHVILLE TN 37204

$615458-4168$

JOHNSON JOE

N $\&$ OEPT OF AGRIC

OIV OF MAF.KETING

PO $80 \times 40627 / M E L R C S E$ STA

NA SHVILLE. TN 37204

$901852-4361$
MARTIN DOUGLAS B

OIV OF MARKETING

PO BOX 40627 MMEROSE STA

NASHVILLE, TN 37204

AUNDEN, OAKEY

W. M OEPT OF AGRIC

OIV OF MAR KETING

PO BOX 40627 /MELROSE STA

NASHVILLE. TN 37204

$615892-6767$

NORMAN: EUGENE

W. M DEPT OF AGRIC

DIV OF MARKETING

PD BOX 40627 MELROSE STA

NASHVILLE: IN 37204

$901364-2075$

PARK \& $P$

W $M$ DEPT OF AGRIC

DIV OF MARKETING

PO BOX 4062 T/MELROSE STA

NASHVILLE IN 37204

$901586-7936$

PARKSO ROBERT S

W $\&$ M DEPT DF AGRIC

OIV OF MARKETING

PO BOX 40627 MELROSE STA

NASHYILLE TN 37204

$901772-2413$

PITTMAN: CATHRYN F

METROLOGIST

DEPARTMENT OF AGRICULTURE

PO BOX 40627 MELROSE STATION

NASHYILLE. TN 37204

$615360-0159$

QUALLS, JAHES L

DE MDEPT OF ÁGRIC

PO BOX 406 ? T M MELROSE STA

NASHYILLE. IN 37204

$615696-8116$

RHODES. DAVID $K$

$W \& M D E P T$ OF AGRIC

DIV OF MAR.KETING

PO BOK 40627 /MELROSE STA

NASHVILLE. TN 37204

$901549-3875$

SCOTT OANNY RAY

W E DEPT OF AGRIC

DIV OF MARKETING

PO BOX \$0627/NELROSE STA

NASHVILLE TN 37204

$901549-9446$

SMITH, EDWIN L

SE MDEPT OF AGRIC

DIV OF MARKETING

NASHVILLE, JN 37204

$615526-5745$

SMITH JR CARL

$W \& M$ DEPT OF AGRIC

DIV OF MAKKETING

PO BOX 40627 JMELROSE STA

NASHVILLE. IN 37204

$615581-0543$

THCMP SON JAMES

WE M DEPT OF AGRIC

DIV OF MARKETING

PO BOX 40627 MELROSE STA

NASHYYLLE TN 37204

$615759-7602$ 
VENABLE LLESTER C

AENA DEPT OFAGRICULTURE 40627 MELROSE STATICN

NASHVILLE TN 37204

WA SHBURN, RAYFORD

H Y M DEPT OF AGR IC

DIV OF MARKETING

PO BOX $40627 / M E L R O S E$ STA

NASHVILLE TN 37204

$901855-0728$

WILLIAMSE ROBERT G

DIVISION DF MARKETING

PO BOX 40627 MELROSE STA

NASHVILLE IN 37204

$615360-0144$

WOQDS E EDDIE

DIV OF MARKETING

PO BOX 40627/MELROSE STA

NA SHVILLLES TN

KNOX COUNTY

HATMACHER, CATHY

CODE ADMIN E INSPECTION

400 W MAIN AVE

KNOXVILLE TN 37902

SHELBY COUNTY

MASSEY VERNON L

SEAELER CCUNTY GOVERNMENT

QI 4 JEFFERSON

MEMPHIS. TN 38105

$901528-3456$

\section{CITY: CHATTANOOGA}

HILL MERRILL C

SUPERYISOR DF WEIGHT \& MEASURE

CITY CF CHATTANOOGA

216 CITY HALL

CHATTANOGGA: TN 37402
$615757-5169$

CITY: KNOXYILEE

RUTHERFQRD, JOHN E

SEALER WEIGHTS \& MEASURE

COT OF KNOXY ILLE

800 CHURCH AVE

KNOXVILLE TN 37915

CITY: MEMPHIS

FRENCH, MICHAEL

MANAGER

WEIGHTS E MEASURES BUREAU

MBR

MEMPHIS. TN 38105

$901528-2906$

HALL B BERT

MEMPHIS WGTS E MEASURES

590 MASHINGTON

$901528-29063105$
MBR

LEONARD JAMES W

MEMPHIS WEIGHTS 6 MEASURES

590 WASHINGTON

MEMPHIS. TN 38105

$901528-2906$

RUSSEL HUBERT B

INSPECTOR

MEMPHIS WE IGHTS \& MEASURES

590 HASHINGTON ST

MEAPHIS. TN 38105

$901528-2906$

MBR

CITY: NASHVILLE

NOLEN\& GEORGE E

SEALER OF WEIGHTS \& MEASURES METRO GOVT

300 DEMONBREUN ST

NASHV ILLE: TN 37215

TEXAS

\section{STATE}

BAMSCH, HAYNE

DEPT OF AGRICULTURE

METROLOGY LABORATORY

2935 WES THOLLOW OR

HOUSTON. TX 77082

$713870-9911$

BOYD. BILL C

METRCLOGIST TRAINEE

DEPT OF AGRICULTURE

1 I9 CUMBERLAND RD

AUST IN TX 78704

MBR

BROWN , ROGER C

METROLOGIST TRAINEE

DEPT OF AGRICULTURE

119 CUMBERLAND RD

AUSTIN. TX 78704

$512462-1441$

ESKEW. JAMES H

CHI EF METROLOGIST ST OF TEXAS

TEXAS DEPT GF AGRI CULTURE

119 CUMBERLAND RD

AUSTINO TX 78704

$512462-1441$

FORESTER PATR ICK

METROLOGIST

TEXAS DEPT OF AGRICULTURE

19 CUMB ERLAND RD

AUSTINO TX 78704

$512462-1441$

JUSTICE JASON

DEPT OF AGRICULTURE

METROLOGY LABDRATORY

4502 ENGLEWOOO AVE

LUBBOCK, IX 79414

806 799-B555

PRICE, EDWIN J

ENFORLENENT COCROINATOR

DEPT OF AGRICULTURE

PO BOX 12847

AUSTIN, TX 78711

QUICKSALL BILLCULTURE

$P$ BOX 12847

AUSTIN. TX 78711
$512463-7602$ 
RALEIGH. SUSAN

DIRECTOR OF CONSUMER SERVICES $X$ DEPT DF AGRICULTURE PQ BOX 12847 AUSTIN, IX 78711

$512463-7602$

SMITH。 SAM D

METROLOGY PROGRAM ADMIN

TX DEPT OF AGRICULTURE

19 CUMBERLAND RD

AUSTIN TX 78704

$512462-1441$

\section{IITY: ARLINGTON}

SPIRES JAMES H HES INSPECTOR WETY OF CALLAS CONSUMER SERV

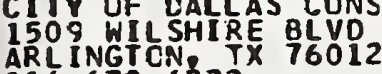
$214670-6393$

\section{ITY: DALLAS}

HENNINGER - RICHARD G

WE IGHTS E MEASURES INSPECTOR CITY OF DALLAS DEPT OF W 32 M DALLAS. TX 75203

$214948-4400$

HERMANSON JOHN R

CITY OF DALLAS DEPT OF HE 320 EAST JEFFER SON RIDOM 312 DALLAS. TX 75203

$214948-4400$

MARTIN, DANIEL

ASSISTANT DIRECTOR 320 EAST JEFFERSON ROOM 312 DALLAS: TX 75203

$214948-4400$

\section{CITY: FORT HORTH}

MALMBERG • JOHN R MALMBERG JOHN R
CONSUAER PRODUCTS I NSPECTOR
CITY OF FORT WDRTH IOOO UNIVERSITY DR RM 208 FORT WORTHE TX 76107 817 870-7571

\section{RICE JR . MASON G \\ CONSUMER PRDDUCIS INSPECTOR RM 208 FORT WORTH IX 76107 \\ $817870-7270$}

WAT SON, DAVID

CONSUMER PRODUCTS SUPERVISOR

MBR
NCHM

CITY OF FORT WORTH TEXAS

1800 UNIVERSITY ROOM 208

FORT WORTH TX 76107

$817870-7572$

STATE

BAI GARF

WE IGHTS E MEASURES

DEPARTMENT OF AGR ICULTURE

350 N REDWOOD RO

SALT LAKE UT 84116

MBR

NCWM

MBR

MBR

MBR

NCWM

MBR

NCWM
CRANE RICHARD

WEIGHIS \& MEASURES

DEPARTMENT OF AGRICULTURE 350 N REDWCDD RD

SALT LAKE $533-5459$ UT 84116

CROOK HAR YEY

WEIGHTS \& MEASURES

DEPARTMENT OF AGR I CULTURE 350 N REDWOOD RD

SALT LAKE UT 84116

GaLE CLAUDIA

WEIGHTS \& MEASURES

CEPARTMENT DF AGRI CULTURE $350 N$ REDWOOD RO

SALT ${ }_{53}$ AKE

LEAVITT JIM

WEIGHTS 6 MEASURES

DEPARTMENT OF AGR I CULTURE

$350 \mathrm{~N}$ RECWCDD RD

SALT 801 LAKE U3 UT 84116

LEHMIT D. DON

WEIGHTS E MEASURES 350 N REDWOOO RD

SALT LAKE UT 84116

MAYS OAN

WEIGHTS \& MEASURES

DEPARTMENT OF AGRICULTURE $350 \mathrm{~N}$ REDWOOD RD

SALT 501 LAKE U UT 84116

MCKAY. DAYID

WEI GHTS \& MEASURES

DEPARTMENT OF AGR I CULTURE 350 N REDWOOD RD

SALT LAKE, UT 84116

$801533-5459$

MITCHELL: CONNELL

DEPARTMENT OF AGR I CULTURE $350 \mathrm{~N}$ REDWOOD RD SALT LAKE, UT 84116

$801533-5459$

NIELSQN • GARY

HEIGHTS $\&$ MEASURES

DEPARTMENT OF AGR I CULTURE

$350 \mathrm{~N}$ REDHOOD RD

SALT LAKE UT 84116

$801533-5459$

NORMAN. SID

HEIGHTS \& MEASURES

DEPARTMENT OF AGR I CULTURE $350 \mathrm{~N}$ REDWOOD RD

SALT LAKE. UT 84116

$801533-5459$

RICO. HUGO

HEIGHTS \& MEASURES

DEPARTMENT OF AGR I CULTURE

$350 \mathrm{~N}$ REDWOOD RD

SALT LAKE: UT 84116

STEPHENS EDISON J

DEPUTY COMMISS IONER

DEPT OF AGRI CULTURE

350 NORTH REDWOOD RCAD

SALT LAKE. UT 84116
MBR NChM 
WALKER: KEN

WE IGHT'S G MEASURES

DEPARTMENT OF AGRICULTURE

$350 \mathrm{~N}$ REDWOOD RO

SALT LAKE UT 84116

$802533-7552$

\section{VER.MONT}

\section{STATE}

ATHOOO, KEN

INSPECTS \& MEASURES RETAIL INSP

3 SANFORD RD

BALLOU GREGORY

WEIGHTS E MEASURES RET AIL INSP

BELLOWS FALLS. VT 05101

BRINK, TRAFFORO F

DIR WH S \& MEAS E RETAIL INSP

116 STATE ST

MONTPELIER: VT 05602

BUMP FRANK

INSPECTCR

VT CEPT OF AGRICULTURE

23 GREEN SQUARE

PRCCTOR. VT 05765

CIOFFI. RAYMOND P

METROLOGIST

OIVISION OF WEIGHTS ANO MEAS

1 I STATE ST

MONTPELIER VT 05602

FI SHER, RONALD

INSPECTOR/W E M RETAIL INSP 8 ENR IGHT AVE

WINOSOR. VT 05089

HOWARD, ROBERT L

INSPECJOR/W E M RETAIL INSP

VERAONT OEPT OF AGR ICULTURE

$R R$ FI BOX 398-G

WAITSFIELD. VT 05673

MARCKRES, HENRY

INSPECTOR

WEIGHTS \& MEASURES RETAIL INSP

CRAFTSBBURY。VT 05826

MARTELL BRUCE

SUP WEI GHTS \& MEAS RETAIN INSP

VERMONT CEPT OF AGR ICULTURE

16 STATE ST

MONTPELIER VT 05602

PETERSON, KIMBERLY

INSPECTOR OF AGRICULTURE

HATERBURY, VT 05676

\section{VIRGIN ISLANDS}

\section{STATE}

DYER HOWARD R

DEPUTY DIRECTOR HEM

CNSUMER SVC ADMINISTRATION

作

CHRISTI ANSTED. VI 00820
$809773-2226$
LEBRON ANGEL

CONSUMER SERVICES DIVISION

GOLOEN ROCK SHOPPING CENTER

CHRISTIANSTED ST. VI D0820

PENN LOUIS

ACT ING DIRECTOR

CONSUMER SERVICES CIVISION

GOLDEN ROCK SHOPPING CENTER

CHRISTIANSTED ST. VI 00820

809 774-3130

VIRGINIA

STATE

ANDERSON: M I

WEIGHTS \& MEASURES DIV

STATE DF VIRGINIA

P D BOX 1163 RM 403

RICHMONO VA 23209

MBR $804786-2476$

NCWM

MBR

NCWM

MBR

NCWM

MBR

MBR

NCWM

BAL DWIN. H D

WEIGHTS $\varepsilon$ MEASURES DIV

STATE DF VIRGINIA

210 CHURCH AVE SW

RÓANOKE VA 24011

03 982-7353

BEVINS, F A

WEIGHTS \& MEASURES OIV

STATE OF VIRGINIA

210 CHURCH AVE SW

ROANOKE. YA 24011

$703982-7353$

BOWERS. JAMES $P$

WEIGHING EQUIPMENT SUPERVISOR

VA DEPT HWYS \& TRANSPORTATION

1221 BRDAD STREET

RICHMOND VA 23219

$804786-6785$

BUNCH ${ }^{R} E$ MEASURES DIV

STATE OF VIRGINIA

$P$ D BOX 1163 RH 403

RICHMOND, VA 23209

$804786-2476$

CAIN, MARION W

METROLLOGIST AGR ICULTURE

PO BOX 1163 RM 403

RICHMOND VA 23209

$804786-2476$

CORVINE L P

HEIGHTS $\&$ MEASURES DIV

STATE OF VIRGINIA

210 CHURCH AVE SW

ROANOKE Y YA 24011

MBR

COX ${ }^{R}{ }^{M}$

STATE $E$ MEASURES DIV

O BOX 1163 RM 40

RICHMOND 113 VA 23209
$804786-2476$

DEMORY: $R$

WEIGHTS 6 MEASURES DIV

STATE OF VIRGINIA

210 CHURCH AVE SW

ROANŎKE. VA 24011

$703982-7353$ 
IGGS ${ }^{G}{ }^{G}$ WEIGHTS E MEASURES MBR

0 BOX II63 ROOM 403

ICHMOND, VA 23209

$104786-2476$

=INK, R 2

ISST MA INTENANCE ENGINEER

L22 IEPAST BROAD ST

RICHMOND, VA

FR I CND. S

WEIGHTS \& MEASURES DIV

STATE OF VIRGINIA

210 CHORCHA 24011

ROA $982-7353$

HARGRAYE, I

WEIGHTS $E$ MEASURES DIV

STATE OF VIRGINIA

RICHMOND YA 23209

$804786-2476$

JOHNSON LAWRENCE M

ASSOC SEALER WEIGHTS MEAS

3959 PENDER DRIVE SUITE 200

FAIRFAX VA 22030

$703691-2388$

LYLES, JAMES $F$

CHIEF IVIA WEIGHTS E MEASURES

$P$ BOX 1163 ROOM 403

RICHAOND, VA 23209

$804786-2476$

MCDCUGLE, R H

WEIGHTS $E$ MEA SURES DIV

STATE OF VIRGINIA

P O BOX 1163 RH 403

$804786-2476$

MICHAUX, JE

HE GHTS C MEA SURES DIV

STATE OF VIRGINIA

P.C BOX 1163 RM 403

RICHMOND VA

MUSSER R RE
REGIONAL SUP ERYISCR
VIRGINIA WEIGHTS \& MEA SURES

$P$ OBOX 1163 ROOM 403

RICHMOND VA 23209

$804786-2476$

PARKS $T R$

HEIGHYS $\&$ MEASURES DIV

STATE OF VIRGINIA

$P$ OOX 1163 RM 403

RICHMOND VA 23209

$804786-2476$

PARTLOW P $P$

WEICHTS \& MEASURES DIV

STATE DF VIRGINIA

210 CHURCH AVE SH

ROANOKE VA 24011

$703982-7353$

PORTER, G E

WE IGHTS \& MEASURES DIV

STATE OF VIRGINIA

210 CHURCH AVE SW

ROANOKE. YA 24011

$703982 \div 7353$

MBR

MBR

ABR

MBR

ACWH
ROGERS S SA SUPERVISOR

VIRGINEA WEIGHTS E MEA SURES

MBR

O BOX 1163 ROOM 403

RICHMOND YA 23209

SADLER ' SÜPER VISOR

VIRGINIA WEIGHTS \& HEA SURES

$P$ BOX 1163 ROOM 403

RICHMOND YA

MBR

$804786-2476$

SANDERS, J G GURES DIV

STATE OF VIRGINIA

$P$ O BOX 1163 RM 403

RICHMOND VA 23209

SHELTON RICHARD H

SUPERVISOR CUANTITY STANDARDS VA WEIGHTS $C$ MEASURES SECTION PO BOX 1163 ROOH 403

RICHAOND YA 23209

804 786-2476

MBR

NCWM

SOUTHALL DBILLY "AGRICULTURE

1 IOO BANK ST

R04 786-3534 23219

NCWH

TAYLOR, S

MEIGHTS $\&$ MEASURES DIV

STATE OF VIRGINIA

210 CHURCH AVE SW

ROANOKE. VA 24011

$703982-7353$

THONAS. $R V$

WEIGHTS \& MEASURES DIV

STATE OF VIRGINIA

P BOX 1163 RH 403

RICHMOND VA

B04 786-2476

HILSON: P A

WEIGHT' - MEASURES DIV

STATE OF VIRGINIA

P C BOX 1163 RM 403

RICMMCND VA $23209^{\circ}$

$804786-2476$

FALRFAX COUNTY

ELDER, JAHES LEO

SEALER HEIGHTS \& MEASURES

DEPT OF CONSUMER AFFAIRS

3959 PENDER DR

$703691-32$ I4 22030

CITY: NORFOLK

GARNSE KEITH IAHTS 6 MEASURES

CITY OF NORFOLK

CITY HALL ROOA 802

NORFOLK. $Y A$ 23501

$804441-2409$

CITY: VIRGINIA BEACF.

MESSING ${ }^{R}$ EE DEPT OF PERMIS E INSPECTIONS MUNICIPAL CENTER

VIRGINIA BEACH, VA 23456

$804427-4421$
MBR

MBR

NCWM

NCWM 
ALLEN JOHN W

WE IGHTS E MEA SURES INSPECTOR

STATE DF WASHINGTON

SEATTLE W WA 98166

CAMMEL JAMES H

INSPECTOR III/METROLOGIST

SECTION OF WEIGHTS E MEASURES

406 GEPJERAL ADMIN BLDG

OL YMPIA. WA 98504

$206753-5042$

DE LANEY, STUART

CHIEF

DEPT OF AGR WEIGHTS 6 MEASURES 406 GENEPAL ADMINISTRATION BLD DLYMPIA. HA 98504

$206753-5042$

FLUHARTY ARTHUR

INSPECTOR I WEIGHTS \& MEASURES

406 GENERAL ADMIN BLDG

OL YMPIA. WA 98504

$206753-50 \% 2$

HARDER GARY HASH UTILITIES $E$ TRANS COMM

HIGHWAY-LICENSE BLDG

OL YMPIA. HA 98504

$206753-6434$

HARDINO M R

INSPECTOR I

406 GENERAL ADMIN BLDG

DL YMP IA. WA 98504

$206753-5042$

HOWELL O YAYNE

INSPECTOR I

406 GENERAL ADMIN BLDG

OL YMPIA. WA 98504

$206753-5042$

KANOUSE, LARRY

INSPECTYR I

WEIGHTS \& MEASURES

406 GENERAL ADMIN BLDG

OL YMPIA. WA 98504

$206753-5042$

MARTINO JOHN

INSPECTOR I

406 GENERAL ADMIN BLOG

DL YMPIA. WA 90504

$206753-5042$

NEFF THOMAS A

PROPERTY MGMT COMMANDER

WASHING TCN STATE PATROL

MBR

4242 MARTIN HAY

OLYMPIA. WA 98504

$206753-6854$

RISE DIANE

SECTION OF WEIGHTS \& MEASURES

406 GENERAL ADMIN BLDG

OLYMPIA. WA 98504

? $06753-504$ ?

MBR

MBR
MBR

NCWM

SCHLEGEL, BOB

INECTION OF WEIGHTS \& MEASURES

406 GENERAL ADMIN BLDG

DLYMP IA. WA 98504

$206753-5042$

SCHHIDT GEORGE

INSPECTOR I

SECTION DF WEIGHTS 8 MEASURES

406 GENERAL ADMIN BLDG

OLYMPIA. WA 98504

$206753-5042$

NCWH

NCWM

CITY: EVERETT

ANDERSON, KRISTIE

SEALER OF HEIGHT

G MEASURES

3200 CEOAR ST

EVERETT, HA 98201

$206259-8745$

CITY: SEATTLE

BJORNSSON. PAUL

INSPECTOR WEIGHTS 6 MEA SURES

DEPT OF LICENSES \& CONS AFF

805 S DEAR BORN ST

SEATTLE: WA 98134

FEAGAN, BRUCE

INSPECTOR WEIGHTS $\&$ MEASURES DEPT OF IICENSES $E$ CONS AFF 805 S DEARBORN ST SEATTLE. WA 98134

$206 \quad 625-2717$

GARLINGTON . RALPH

INSPECIOR WEIGHTS $\&$ MEASURES

DEPT DF LICENSES 6 CONS AFF

805 S DEAR BORN ST

SEATTLE, WA 98134

$206 \quad 625-2717$

MCFARLANE, STERLING

SUPERVISDR WEIGHTS \& MEASURES

DEPT DF LICENSES E CONS AFF

ROOM 1026004 TH AVE

SEATTLE. WA 98104

$206 \quad 625-2717$

SEL ANDER A R WEIGHTS $E$ MEASURES 805 S DEARBORN ST SEATTLE: HA 98134

NCWM

SETTERHOLH. DAVID E

INSPECTOR WEIGHTS \& MEASURES

DEPT OF LICENSES \& CONS AFF

$\$ 05$ S DEARBORN ST

SEATTLE. WA 98134

$206625-2717$

TOLLEFSON, LINDA $G$

DEPUTY SEALER

805 S DEAR BORN ST

SEATTLE: WA 98134 


\section{CIIY: SPCKANE}

ILLEN, GILBERT $R$

SEALER OF SPOKANE

IEST 808 SPOKANE FALLS BLVD

SPOKANE, WA 99201

$509456-4306$

JONES, RALPH

DIV OF WEIGHIS AND MEASURES

N 7306 MAHR COURT

SPOKANE WA 99208

$509467-0494$

SCHMALL \& ALEX M

DEPUTY SEALER WEIGHTS EMEAS

WE IGHTS $\&$ HEASURES

54431 KRIKEN DR

SPOKANE WA 99204

$509624-9329$

TTY: TACOMA

BOOKER, RICH

INSPEC TOR HEIGHTS $E$ MEASURES DEPT OF PUBL IC HORK SICONS DIV 930 TAC OMA AVE S/4TH FL

TACCMA WA 98402

SCHAEFER JAMES C

CITY OF TACOMA

930 TACCMA AVE S

$206593-4780$

SPARLING TIM

INSPECTCR HEIGHTS $\&$ MEASURES DEPT OF PUBL IC WORK SICONS DIV 930 TACOMA AVE S/4TH FL

TACCMA WA 98402

$206591-5760$

\section{IIR.GINIA}

;TATE

ANGELL KARL H

METROLOBGIST

WEIGHTS E HEA SURES DIVISION $570 \mathrm{~W} \mathrm{MAC} \mathrm{CORKLE}$ AYE

$304727-5781$

HOLT JCHN A

STATEWIDE SUPERYISOR

WEIGHTS E MEASURES
570 WEST MACCORKLE AVE
ST ALBANS WV 25177

$304727-5781$

RARDIN: JAMES

DEPARTMENT OF LABOR

570 MACCORKLE AVE

$304727-5781$

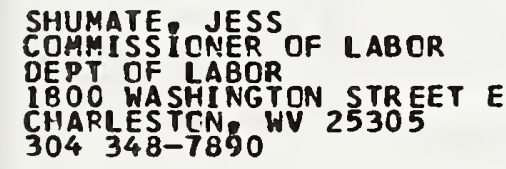

MBR

NCWM

MBR

MBR

NCWM

MBR

NCWM

MBR
STATE

BARKOW: GORDON

WEIGHTS E MEASURES

HISCONSIN DEPT OF AGRICULTURE $200 N$ JEFFERSON SUITE 146 A

GREEN BAY WI 54301

\section{BRIMMER, KERRY \\ STATE OF WISCONSIN \\ 927 LOR ING AVE \\ ALTOONA. WI 54720}

BROWN, MIKE

NEOWN: MIKE

WISCONSIN DEPT OF AGRICULTURE 200 N JEFFER SON SUITE 146 A

GREEN BAY, WI 54301

$414436-4087$

BURKHARDT: WILLIAM E

LABORATORY SUPERVISOR

WISCONS IN DEPT OF AGRICULTURE

4702 UNIV AVE BOX 7883

MADISON, WI 53707

$608267-3510$

CALOGERC, LOUIS

P O BOX I2OI
927 LOR ING AVE
ALTOONA! WI 54720
$715839-3797$

DONAHUE DON

STATE OF WISCONSIN

200 N JEFFERSON SUITE $146 \mathrm{~A}$

$414436-4087$

HANSEN: W'

HISCONSIN DEPT DF AGRICULTURE 801 HADGER RD-BOX 8911

MADI SON. WI 53708

HOFFMAN. JAMES $P$

WEI GHTS E MEASURES INSPECTOR

$M B R$ WISC DEPT OF AG TRADE AND CP

NCWM 5OG SOUTH PRAIRIE ST

STOUGHTON 266-7222

JANSEN S JANE

MBR WI DEPT AGRI TRADE CONSUMER PR 801 BADGER RD-BOX 8911

MAOISON. WI 53708

$608266-7241$

KAELIN, GEORGE

STATE OF HISCONSIN

927 LORING AVE

ALTOONA. WI 54720

$715839-3797$

KILIAN. ELMER

WEIGHTS $\&$ MEASURES INSPECTOR

TRADE E CONSUMER PRCTEC TION

10320 SILVER SPR ING OR

MILWAUKEE. WI 53225

MBR

NCWM 
KOSLR, WALLACE

WISCONS IN DEPT OF AGRICULTURE BOI W BADGER RD-BOX 8911

MADISON. WI 53708

608 266-5642

LEMKE ALDEN

WEIGHTS \& MEASURES

HISCONSIN DEPT OF AGRICULTURE BO W BADGER RD-BOX 8911

MADISON HI 53708

608 266-5642

NELSCN. RUSSELL

WE IGHTS \& MEASURES 801 W ADGER RD-BOX 8911

MADISCN WI 53708

608 266-5642

PARX, BOB

CP COMPLIANCE OFF ICER

WI SCONS IN DEPT OF AGRI CULTURE 801 BADGER RD-BCX 8911

MADISON: WI 53708

$608267-9512$

PORTER, ALAN

WEIGHTS $E$ MEASURES

WI SCONSIN DEPT OF AGRICULTURE $801 W$ BADGER RD-BOX 8911

MADISON. WI 53708

$608266-7244$

QUA IDE MARGE

WW REG SUPY

927 LOR ING AVE

ALTCONA. WI 54720

$715 \quad 839-3797$

RICHTER, HARVEY

WE IGHTS \& MEASURES

HI SCONSIN DEPT OF AGRICULTURE $10320 \mathrm{~W}$ SILVER SPRING DR

MILWAUKEE WI 53225

$414438-4848$

ROBARGE -WILLIAM

WEIGHTS E MEASURES

WISCONSIN DEPT OF AGRICULTURE 200 JEFFER SON SUITE $146 \mathrm{~A}$ GREEN BAY, WI 54301

$414436-4087$

SOBERG DONALD J

WI SCONSIN DEPT OF AGRICULTURE 801 W BAGER RD P O BOX 8911 MADISON. WI 53708

608 266-7220

SUSCHIL PATRICIA

SE REG SUPV

WI SCONNS IN DEPT OF AGRICULTURE MILWAUKEE WI 53225

$414438-4848$

CITV: APPLETON

RICHTER JAMES L

CITY SEALER OF WEM

CITY OF APPLETON-INSPECTION DT 1024 SOUTH LAWE STR

$414735-5587$
CITY: FOND DU LAC

KNIPPLE RCBERT

SEALER OF WEIGHTS AND MEASURES CITY OF FOND DU LAC

PO BOX 150

FIN $929-2952$ : 54935

CITY: FRANKLIN

WEITKUNAT. EDHARD A

SEALER OF WEIGHTS \& MEASURES

CITY OF FRANKL IN

7862 CHAPEL HILL CTE

FRANKLIN 14 T25-1927

CITY: GREEN BAY

KRYZANEK, DANIEL E

CITY SEALER

OEPT OF WEIGHTS \& MEASURES

CITY HALL $100 \mathrm{~N}$ JEFFER SON

GREEN BAY WI 54301

$414436-3665$

CITY: GREENFIELD

ZARNIK, DANIEL A

CITY SEALER

CITY OF GREENF IELD WI

561 S 41 ST

$414421-3345$ WI 53221

CITY: KENOSHA

BENN HARRY $M$

INSPECTOR

CITY OF KENOSHA

MUNICIPAL BLOG 1625 52ND ST

KENOSHA. WI

CITY: LA CROSSE

HANSON. ALBAN E

CITY SEALER INSPECTION DEPT

C ITY OF LA CROSSE

$505 \mathrm{~N}$ 6TH ST

LA CROSSE WI 54601

MBR

NCWM

CITY: MADISON

PETERSON. CHERYL

CITY OF MAOISON

4602 SVAMORE AVE

MADISON. WI 53714

608 266-4780

CITY: MAR SHFIELD

TYROLT, VIRGIL D

SEALER OF WEIGHTS \& MEASURES CITY OF MARSHF IELD PO BOX 727 MARSHFIELD. WI 54449

$715387-1344$

CIIY: MENASHA

PRAST FRANCIS P

CITY SEALER

CTY OF MENASHA WI 54952

737 STATE ST

MENASHA: WI 54952 


\section{IYY: MILWAUKEE}

VINRUDEg MARK

TEALER CF WEIGHTS \& MEASURES ITY OF MILWAUKEE

1630 W MILL RD

1IL LAUKEE: WI 53218

GUNDER SON, HARRY

SEALER OF WEIGHTS $\&$ MEASURES

CITY OF MILWAUKEE

5325 NORTH 74 ST
MILLAUKEE. WI 53218

HOFMAN GORDON L \& MEASURES

CITY OF MILWAUKEE

$3363 \mathrm{~N} 51$ ST BLVD

MILWAUKEE $278-367453218$

ROBINSON, LOYCE C

DEPUTY CITY SEALER

BUREAU OF CONSUMER PROTECTION

841 N BROADWAY RM 105 MUN BLDG

MILWAUKEE WI 53202

$414278-3674$

WALI KENNETH F

SEALER OF WEIGHTS \& MEASURES

CIIY OF MILWAUKEE

7630 W MILL RD

MILWAUKEE. WI 53218

$414278-3674$

ZEMRE PAULE W

CIIY OF MILWAUKEE

$763 \mathrm{C} W \mathrm{MILL}$ RD

MILKAUKEE WI 53218

ITY: OSHKOSH

ERDMANN $\angle E E$

CITY SEALER HEI GHTSEMEA SURES

CITY OF CSHKOSH

215 CHURCH AVE

$414236-5047$

ITY: RACINE

MECHE RUSS

CI TY OF RAC INE

730 WASHINGTON AVE

RACINE: WI 53403

ITY

SHEBOYGAN

BEL INKY , SQLOMAN

SEALER OF WEIGHTS $\&$ MEASURES

$709 \mathrm{~N} 7 \mathrm{TH}$ ST

SHEBOYGAN. WI 53081
$414459-3488$

VANDE BERG $\angle A R R Y$ A

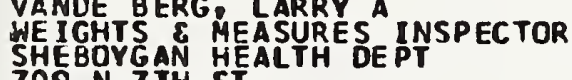

709 N 7 TH ST

SHEBOYGAN, WI 53081

$414459-3485$

\section{CITY: SPARTA}

BURKE: L LEO $D$

CITY SEALER

WEI GHTS ME SURES

SPI TARTA. ST 54656

$608269-6511$

CITY: STURGEON BAY

MEYER, WAYNE

ASST SEALER

CITY OF STURGEON BAY

360 NEBRASKA ST

STURGEON BAY. WI 54235

$414743-6913$

TAUBE $\triangle O H N H$

CITY SEALER

CITY OF STURGEON BAY

360 NEBRASKA ST

STURGEON BAY, WI 54235

$414743-6913$

MBR
NCWM

MER

CITY: WEST ALLIS

ADAMS. JANE

WEST ALL IS HEALTH DEPT

7120 W NAT IONAL AVE

HEST ALLIS: HI 53214

BUEGE, RONALD $M$

DEPUTY CITY SEALER

HEST ALLIS HEALTH DEPARTMENT

7120 W NATIONAL AVE

WEST ALLIS: WI 53214

MBR

NCWM

EGGER S: ER IC $K$

ASST CITY SEALER

WEST ALL IS HEALTH CEPARTMENT WEST ALCIS WI 53214

$414476=4340$

MURRAY. ROBERT

SEALER

WEST ALL IS HEALTH DEPARTMENT

7120 W NAT IONAL AVE

WEST ALLIS: HI 53214

MBR

ZENTGRAF JOHN

HEST ALLIS HEALTH OEPT

7120 W NAT IONAL AVE

WEST ALLIS: WI 53214

$414476-4340$

WYOMING

STATE

ANDER SON HAROLO

WYOLMING DEPT OF AGRICULTURE 2219 CAREY AVE

CHEYENNE WY 82002

$307777-7321$

BURCHAM ART

C E C INSPECTOR AGRICULTURE

2219 CAREY AVE

CHEYENNE WY 82002

307 777-7321 


\section{CARLILE TERRY \\ PO ${ }_{B O X}{ }^{2}{ }^{2} 1$ \\ 3 SMOT WY 83126}

GEREER Y YICTOR

WYOMING DEPT OF AGR ICULTURE

2219 CAREY AVENUE

CHEYYNTE 9 WY 82002

HATTANO BRUCE

C E C SUPVIAREA

WYOMING OEPT OF AGRICULTURE

2219 CAREY AVE

CHEYENNE WY 82002

$307777-7321$

HOVEY, HILLIAM W

MGR CON SUMER/COMPLIANCE DIV WY DEPT OF AGRI CULTURE

2219 CAREY AVE

CHEYENNE WY 82002

JOHNSON VERNON

HEAVY SCALE INSPECTCR

2219 CAREY AVE

CHEYENNE: HY 82002

KELLER: RON

C E C INSPECTOR

WYOMING OEPT OF AGRICULTURE

2219 CAREY AY

$307377-7321$

KOKESH, FREDDIE

22 I9 CAREY ATOR

CHEYENNE HY 82002

$307777-7321$

MICKEL SCN, ALBIE

WYOM SUPVIAREA 2 IGRICULTURE 2219 CAREY AVE

CHEYEYNE HY 82002

MUHLBACH TOM

CYE C INSPECTCR AGR ICULTURE 2219 CAREY AV

CHEYENNE HY 82002

RIEKE, STEVE

WYOMING DEPT OF AGR ICULTURE

2219 CAREY AVE

CHEYENNE 3 HY 82002

STRATTON LINDA

HYO C ING DEPT OF AGR ICULTURE 2219 CAREY AVE

CHEYENNE $H Y$ Y 82002

STUMBOUGH GRANT

C \& E C INSPECTOR AGRICULTURE 2219 CAREY AVE

$307777-332182002$
UHDEN HENRY

C \& C INSPECTOR

CHEYENNE WY 82002

$307777-7321$

MBR

WEBER • RCN

C E C SUPY

WYOMING DEPT OF AGR ICULTURE 2219 CAREY AVE

CHEY T77-3321 82002

WOODWARD KIM

C E C INSPECTOR

WYOMING OEPY OF AGRICULTURE 2219 CAREY AVE

CHEYENNE W WY 82002

307 T7 7321 
SECTION III

FEDERAL GOVERNMENT MEMBERS 

FEDERAL GOVERNMENT MEMBERS

National Bureau of Standards

NN B BARKLEY

ONAL BUREAU OF STANDARDS

IN BLDG ROOM A617

THER SBURG
$921-2401$

ROLL S BRICKENKAMP

GRAM MANAGER

IONAL BUREAU OF STANDARDS

ICE OF WEIGHTS \& MEASURES

THER SBURG. MD 20899

$921-2401$

NDA CAPINO

VINISTRATIVE OFFI CER

IONAL BUREAU OF STANDARDS

ITHER SBURG, MD 20899

I $921-3751$

$\checkmark 10$ E EDGERLY

I EF OFC DOM \& INTL MSMT STDS

ITHER SBURG. MD 20899

$1921-3307$

\section{EPHEN HASKO}

GINEER

TIONAL BUREAU OF STANDARDS

FICE OF WEIGHTS \& MEASURES

ITHER SBURG. MD 20899

i $921-2401$

IN P HEFFERNAN

NNFERENCE COORDINATOR

ITIONAL CONFERENCE ON W $6 M$ BOX 3137

II THERSBURG. MD 20878

JAN A KOENIG

ECHNICAL COORDINATOR

ITHNICAL BUREAU OF STANCARDS

-FICE DF WEIGHTS AND MEASURES

IITHERSBURG. MD 20899

AUL H KRUPENIE

IBORATORY ASSISTANT

ATIONAL BUREAU OF STANDARDS

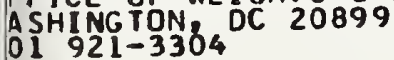

WALTER G LEIGHT

NATIONAL BUREAU OF STANDAROS

OP SP

WA SHING TON 32 DC 20899

KARL NEWELL

DFFICE OF WEIGHTS \& MEASURES

NATIONAL BUREAU OF STANDARDS

GAI THER SBURG. MD 20899

$301921-3677$

\section{HENRY $Y$ OPPERMANN}

GEN PHYS SCI

NATIONAL BUREAU OF STANDARDS

OFFICE CF WTS \& MEASURES

WASHING TON. DC 20899

$301921-2401$

RICHARD N SMITH

TECHNICAL COORDINATCR

MATIONAL BUREAU OF STANDARDS

OFF ICE OF HEIGHTS 5 MEASURES

GAITHERS BURG? MD 20899

$301921-3677$

\section{ALBERT D THOLEN}

ALBERT OF OF WEIGHTS 8 MEASURE

NAT IONAL BUREAU OF STANDARDS

GAITHER SBURG. MD 20899

$301921-2401$

ERIC A VADELUND

SENIOR STANDARDS SPECIALIST

NATIONAL BUREAU OF STANDARDS

GAITHERSBURG. MO 20899

GAL $921-3307$

QTTO K HARNLOF

MANAGER-TECHNICAL SERVICES

INTL MEA SUREMENT STANDAROS

NATIONAL BUREAU OF STA

$301921-2401$

STANLEY I HARSHAH

DIRECTOR OFF OF PRCD STNDS POL

NATIONAL BUREAU OF STANDARDS

GAITHERSBURG. MD 20899

3OL $921-3751$ 

FEDERAL GQVERNMENT MEMBERS

Other Government Agencies

IINA P APPLEBY

IVANNA ARMY DEPOT ACTIVITY 'D COMMANDER/BLDG 127 IVANNA. IL 61074 BBERT L COLLETTE
SOC DIR SCIENCE E TECHNOLOGY JOO M ST NH SUITE 580 A SHING TCN DC 20036 $2296-5131$

TMMANDER

SD-SENACA

ENECA ARMY DEPOT

TTN AMXTM-CE-TO-SE

JMULUS, NY 14541

$07869-1110$

IASELL H CROUCH

IS POSTAL SVC ENGINEER ING

1711 P ARKLAWN DR

IOCKVILLE, MD 20852

उol $443-4810$

\section{AUL HADYKA}

NDUSTRI AL SPECIALIST

ISDA, FGIS

1400 INOEPENDENCE AV RM 0619-S

AASHINGTON. DC 20250

$202382-0262$

JOHN C HAL VERSON

JEPUTY DIR STANDARDIZATION

USDA-FEDERAL GRAIN INSP SERV

I0383 N EXEC HILLS BL/PO 20285

KANSAS CITY, MO 64195

$816348-2484$

\section{LEONARD JARRELL}

OPERATIONS RESEARCH ANALYST

US POSTAL SERVICE ENGINEERING

11711 PARKLAWN DR

ROCKVILLE. MD 20852

$301443-6070$
JOHN LACY

CHIEF SCALES $E$ HEIGHING BRANCH USDA PACKERS OF STOCKYARDS ADM 14 TH $\&$ INDEPENDENCE

WASHING TON, DC 20250

$202475-3209$

DONALD T LIDEN

LAB SUPERVISOR

FEDERAL MILK MARKET ADMIN

2684 WEST ELEVEN MILE RD

BERKLEY, MI 48072

$313548-1603$

JOHN W MCCUTCHEON

DEPUTY ADMINISTRATORY

US DEPT CF AGRICULTURE

$14 T H E$ INDEP SW/R35OE-ADM BLDG HA SHING TON: DC 20250

$202447-3521$

PAUL L PETERSON

INDUSTR IAL SPEC IAL IST

USDA PACKERS \& STOCKYARDS ADM

$14 T H$ INDEPENDENCE SW RM3414S HASHINGT ON DC 20250

$202447-5841$

RICHARD R PFORR

CHIEF-SCALES \& WE IGHING BRANCH USDA EOUIPMENT BRANCH WEIGHING SECTION ROOM 0631 SCUTH BLDG WASHINGTON, DC 20250

$202382-1716$

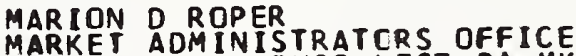
USDA EASTERN OHIO WEST PA MKTG P O BOX 30128

CLEVELAND, OH 44130

$216826-3220$

HERBERT E SHEPARD

FEDERAL MILK MARKET ADMINSTR

90 CANAL STREET 6 TH FLOOR

P O BOX 1478

BOSTON. MA 02205

$617367-6531$

RALPH D SMITH JR

CHEMIST

USDA C E MS DAIRY DIVISION

DALLAS TX 75229

$214243-1504$ 


\section{NCWM MEMBERSHIP APPLICATION}

गease accept my application for membership in THE NATIONAL CONFERENCE ON WEIGHTS AND MEASURES. My check made Jayable to "National Conference on Weights and Measures" is enclosed to cover the annual membership fee of $\$ 35$ for the period ending June 30. Mail to P.O. Box 3137, Gaithersburg, MD 20899. (The membership fee is waived for retirees).

(Please fill in form completely - abbreviate only if necessary)

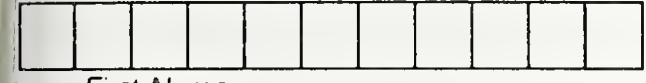

First Name

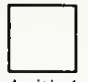

Initial

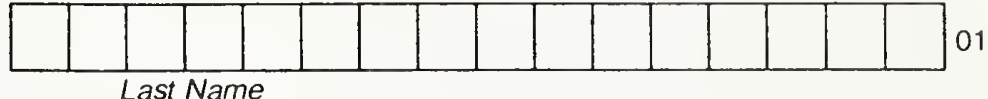

Last Name
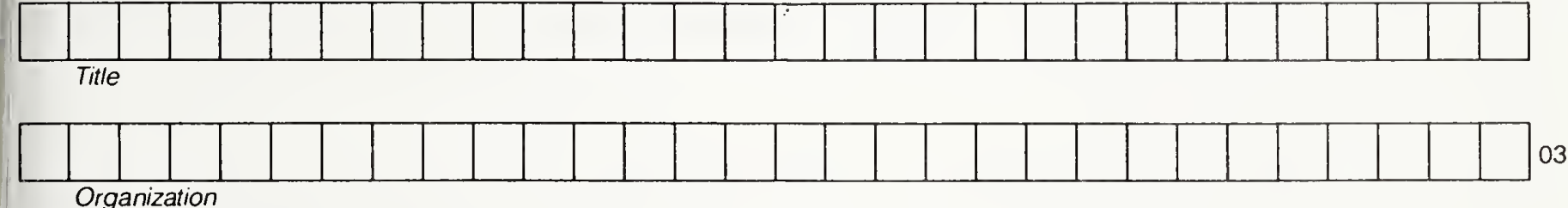

Organization

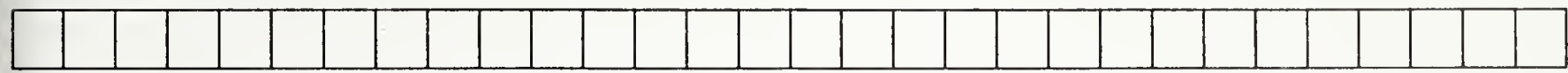

Business Street Address

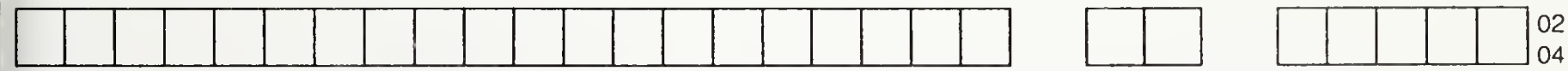

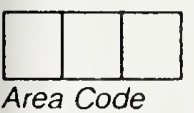

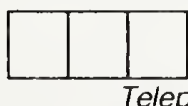

Telephone Number

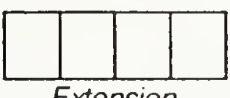

Extension

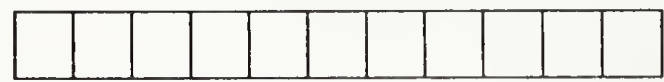

County or Country (as appropriate)

FOR MAIL DISTRIBUTION PURPOSES (check appropriate boxes)

Government Officials

Federal

State (Comm.-Terr.)

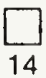

Local (County-City)

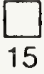

Foreign

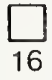

Also check if you are a govern ment "Weights \&. Measures" official

Retired
Private Sector Representatives

Manufacturer (Producer) $\quad \square$

Business, (Retail,

Wholesale, Service)

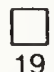

Association (Trade,

Professional)

Educational

Media

Consumer (Public)

Retired
20

21

$\square$

22

23
Your Principal Interest

Packaging: Food

Non-Food

Scales: Weighing

Meters: Petroleum

Other Measurement

Devices

Laboratory Metrology

General 
1 (REV. 2.8C)

U. OEPT. OF COMM.

III.IOGRAPHIC DATA

1. PUBLICATION OR REESRT $8 \%$-3582

E AND SUBTITLE

leights and Measures Directory 1987

HOR(S)

tnn P. Heffernan, Editor

EXFORMING ORGANIZATION (If joint or other than NBS, see instructions)

7. Contract/Grant No.

צTIONAL BUREAU OF STANDARDS

13. DEPARTMENT OF COMMERCE G.ITHERSBURG, MD 20899

)NSORING ORGANIZATION NAME AND COMPLETE ADDRESS (Street, City, State, ZIP)

8. Type of Report \& Period Covered

F INAL

Same as above

IPPLEMENTARY NOTES

Document describes a computer program; SF-185, FIPS Software Summary, is attached.

BSTRACT (A 200-word or less foctual summary of most significant information. If document includes a significant

bliography or literoture survey. mention it here)

This directory is a computer-generated listing of names, addresses, and telephone numbers of state and local weights and measures officials, and National Conference on Weights and Measures members who are U.S. government employees. It is used as a telephone and mailing directory in the weights and measures area.

KEY WORDS (Six to twelve entries; alphabetical order; capitalize only proper names; and separate key words by semicolons)

Directory; jurisdictional listing; weights and measures.

\section{AVAILABILITY}

[X] Unlimited

$\square$ For Official Distribution. Do Not Release to NTIS

$[$ Order From Superintendent of Documents, U.S. Government Printing Office, Washington, D.C. 20402.

[X] Order From National Technical Information Service (NTIS), Springfield, VA, 2216I
14. NO. OF

PRINTED PAGES

132

15. Price 



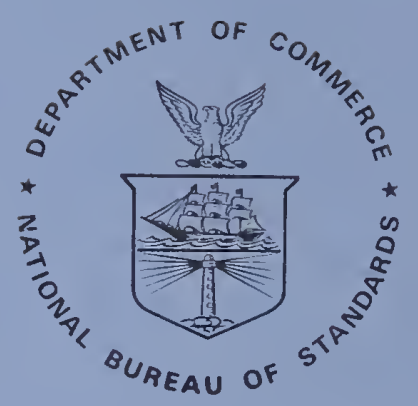

